\section{DEPARTMENT OF HEALTH AND HUMAN SERVICES}

\section{Centers for Medicare \& Medicaid Services}

42 CFR Parts 403, 416, 418, 441, 460, $482,483,484,485,486,488,491$, and 494

[CMS-3346-F; CMS-3334-F; CMS-3295-F]

RIN 0938-AT23

\section{Medicare and Medicaid Programs; Regulatory Provisions To Promote Program Efficiency, Transparency, and Burden Reduction; Fire Safety Requirements for Certain Dialysis Facilities; Hospital and Critical Access Hospital (CAH) Changes To Promote Innovation, Flexibility, and Improvement in Patient Care}

AGENCY: Centers for Medicare \& Medicaid Services (CMS), HHS. ACTION: Final rule.

SUMmARY: This final rule reforms Medicare regulations that are identified as unnecessary, obsolete, or excessively burdensome on health care providers and suppliers. This final rule also increases the ability of health care professionals to devote resources to improving patient care by eliminating or reducing requirements that impede quality patient care or that divert resources away from furnishing high quality patient care. Additionally, this rule updates fire safety standards for Medicare and Medicaid participating End-Stage Renal Disease (ESRD) facilities by adopting the 2012 edition of the Life Safety Code and the 2012 edition of the Health Care Facilities Code. Finally, this final rule updates the requirements that hospitals and Critical Access Hospitals (CAHs) must meet to participate in the Medicare and Medicaid programs. These requirements are intended to conform to current standards of practice and support improvements in quality of care, reduce barriers to care, and reduce some issues that may exacerbate workforce shortage concerns.

\section{DATES:}

Effective date: These regulations are effective on November 29, 2019. The incorporation by reference of certain publications listed in the rule is approved by the Director of the Federal Register as of November 29, 2019.

Implementation dates: The regulations at $\$ 485.641$ regarding Quality Assessment and Performance Improvement Programs (QAPI) in critical access hospitals (CAHs) must be implemented by March 30, 2021.
The regulations at $\S 482.42$ (b) and $\S 485.640$ (b) regarding hospital and critical access hospital (CAH) antibiotic stewardship programs must be implemented by March 30, 2020. FOR FURTHER INFORMATION CONTACT:

For issues related to Regulatory Provisions to Promote Program Efficiency, Transparency, and Burden Reduction, contact Kristin Shifflett, (410) 786-4133.

For issues related to Fire Safety Requirements for Certain Dialysis Facilities, contact Kristin Shifflett, (410) 786-4133.

For issues related to the Hospital and Critical Access Hospital (CAH) Changes to Promote Innovation, Flexibility, and Improvement in Patient Care, contact CAPT Scott Cooper, USPHS, (410) 7869465, Mary Collins, (410) 786-3189, Alpha-Banu Wilson, (410) 786-8687, or Kianna Banks, (410) 786-3498.

SUPPLEMENTARY INFORMATION: We note that this rule finalizes provisions that were proposed in three separate proposed rules that were published in the Federal Register on separate dates. Specifically, we are finalizing the provisions of the following proposed rules, discussed as follows:

- "Regulatory Provisions to Promote Program Efficiency, Transparency, and Burden Reduction," published September 20, 2018 (83 FR 47686);

- "Hospital and Critical Access Hospital (CAH) Changes to Promote Innovation, Flexibility, and Improvement in Patient Care," published June 16, 2016 (81 FR 39448); and

- "Fire Safety Requirements for Certain Dialysis Facilities," published November, 4, 2016 (81 FR 76899)

To assist readers in referencing sections contained in this preamble, we are providing a table of contents.

\section{Table of Contents}

I. Final Rule: Regulatory Provisions To Promote Program Efficiency,

Transparency, and Burden Reduction

A. Executive Summary and Background

1. Purpose

2. Summary of Major Provisions

3. Summary of Costs and Benefits for Regulatory Provisions To Promote Program Efficiency, Transparency, and Burden Reduction

B. Provisions of the Proposed Rule and Analysis and Response to Public Comments

1. Religious Nonmedical Health Care Institutions (RNHCIs)—Discharge Planning

2. Ambulatory Surgical Centers

3. Hospice

4. Hospitals

5. Transplant Centers

6. Home Health Agencies
7. Comprehensive Outpatient Rehabilitation Facility (CORF)— Utilization Review Plan

8. Critical Access Hospitals

9. Community Mental Health Center

10. Portable X-Ray Services

11. Rural Health Clinics (RHCs) and Federally Qualified Health Centers (FQHCs)

12. Emergency Preparedness for Providers and Suppliers

13. Technical Corrections

14. Waiver of Proposed Rulemaking

C. Collection of Information Requirements

II. Final Rule: Fire Safety Requirements for Certain Dialysis Facilities

A. Background

B. Provisions of the Proposed Rule and Analysis and Response to Public Comments

1. 2012 Edition of the Life Safety Code

2. Incorporation by Reference

3. Ambulatory Health Care Occupancies

4. 2012 Edition of the Health Care Facilities Code

5. Technical Corrections

C. Collection of Information Requirements

III. Final Rule: Hospital and Critical Access Hospital (CAH) Changes To Promote Innovation, Flexibility, and Improvement in Patient Care

A. Background

B. Provisions of the Proposed Rule and Analysis and Response to Public

Comments for Hospitals

1. General Comments

2. Implementation Timeframe

3. Non-Discrimination

4. Licensed Independent Practitioner

5. Quality Assessment and Performance Improvement (QAPI) Program

6. Nursing Services

7. Medical Record Services

8. Infection Prevention and Control and Antibiotic Stewardship Programs

9. Technical Corrections

C. Provisions of the Proposed Regulations and Responses to Public Comments for Critical Access Hospitals

1. Organization Structure

2. Periodic Review of Clinical Privileges and Performance

3. Provision of Services (§ 485.635(a)(3)(vii))

4. Provision of Services (§ 485.635(g))

5. Infection Prevention and Control and Antibiotic Stewardship Programs (§ 485.640)

6. Quality Assessment and Performance Improvement (QAPI) Program (§ 485.641)

7. Technical Corrections

D. Requirements for Issuance of Regulations

E. Collection of Information Requirements for Hospital and Critical Access Hospital (CAH) Changes To Promote Innovation, Flexibility, and Improvement in Patient Care

IV. Economic Analyses

A. Regulatory Impact Analysis for Regulatory Provisions To Promote Program Efficiency, Transparency, and Burden Reduction

1. Statement of Need

2. Overall Impact

3. Anticipated Effects 
4. Alternatives Considered

5. Uncertainty

6. Conclusion

B. Regulatory Impact Statement for Fire Safety Requirements for Certain Dialysis Facilities

C. Regulatory Impact Analysis for Hospital and Critical Access Hospital (CAH) Changes to Promote Innovation,

Flexibility, and Improvement in Patient Care

1. Statement of Need

2. Overall Impact

3. Anticipated Effects

4. Effects on Small Entities

5. Alternatives Considered

6. Conclusion

D. Accounting Statement and Table

E. Regulatory Reform Analysis Under E.O. 13771

I. Final Rule: Regulatory Provisions To Promote Program Efficiency, Transparency, and Burden Reduction

A. Executive Summary and Background

\section{Purpose}

Over the past several years, we have revised our requirements, Conditions of Participation (CoPs) and Conditions for Coverage/Conditions for Certification (CfCs) to reduce the regulatory burden on providers and suppliers while emphasizing health and safety. We identified obsolete and burdensome regulations that could be eliminated or reformed to improve effectiveness or reduce unnecessary reporting requirements and other costs, with a particular focus on freeing up resources that health care providers, health plans, and States could use to improve or enhance patient health and safety. We also examined policies and practices not codified in rules that could be changed or streamlined to achieve better outcomes for patients while reducing burden on providers and suppliers of care, and we identified non-regulatory changes to increase transparency and to become a better business partner. In addition, the Centers for Medicare \& Medicaid Services (CMS) and the Department of Health and Human Services (HHS) have reaffirmed their commitment to the vision of creating an environment where agencies incorporate and integrate the ongoing retrospective review of regulations into Department operations to achieve a more streamlined and effective regulatory framework. The objectives were to improve the quality of existing regulations consistent with statutory requirements; streamline procedural solutions for businesses to enter and operate in the marketplace; maximize net benefits (including benefits that are difficult to quantify); and reduce costs and other burdens on businesses to comply with regulations.
In accordance with these goals, we published three final rules that identified unnecessary, obsolete, or excessively burdensome regulations on health care providers, suppliers, and beneficiaries. These rules further increased the ability of health care professionals to devote resources to improving health care by eliminating or reducing requirements that impede quality patient care or that divert providing high quality patient care:

- "Reform of Hospital and Critical Access Hospital Conditions of Participation", published May 16, 2012 (77 FR 29034);

- "Regulatory Provisions to Promote Program Efficiency, Transparency, and Burden Reduction", published May 16, 2012 (77 FR 29002) and;

- "Regulatory Provisions to Promote Program Efficiency, Transparency, and Burden Reduction; Part II', published May 12, 2014 (79 FR 27105).

This final rule is a continuation of our efforts to reduce regulatory burden and is in accordance with the January 30 , 2017 Executive Order "Reducing Regulation and Controlling Regulatory Costs" (Executive Order 13771). We are finalizing changes to the current requirements, CoPs, and CfCs that will simplify and streamline the current regulations and thereby increase provider flexibility and reduce excessively burdensome regulations, while also allowing providers to focus on providing high-quality healthcare to their patients. This final rule will also reduce the frequency of certain required activities and, where appropriate, revise timelines for certain requirements for providers and suppliers and remove obsolete, duplicative, or unnecessary requirements. We believe these policies balance patient safety and quality, while also providing broad regulatory relief for providers and suppliers.

In the proposed rule, we stated that we seek to reduce burdens for health care providers and patients, improve the quality of care, decrease costs, and ensure that patients and their providers and physicians are making the best health care choices possible. Therefore, we solicited public comments on additional regulatory reforms for burden reduction in future rulemaking.

Specifically, we sought public comment on additional proposals or modifications to the proposals set forth in the proposed rule, "Regulatory Provisions to Promote Program Efficiency, Transparency, and Burden Reduction," published September 20, 2018 (83 FR 47686) that would further reduce burden on Medicare and Medicaid participating providers and suppliers and create cost savings, while also preserving quality of care and patient health and safety. We also noted in the proposed rule (83 FR 47686), consistent with our "Patients Over Paperwork" Initiative, that we are particularly interested in improving existing requirements, within our statutory authority, where the requirements as currently written make providing quality care difficult or less effective. We also noted that such suggestions could include or expand upon comments submitted in response to Requests for Information (RFIs) that were included in the 2017 prospective payment regulations for most provider types.

\section{Summary of Major Provisions}

We are reducing regulatory burden on providers and suppliers by modifying, removing, or streamlining current regulations that we now believe are unnecessary, obsolete or excessively burdensome. Specifically, we are finalizing the following revisions:

a. Discharge Planning in Religious Nonmedical Health Care Institutions (RNHCIs)

We have revised the requirements at 42 CFR 403.736(a) and (b) pertaining to a discharge plan. This revision simplifies the discharge process for RNHCIs by requiring them to assess the need for a discharge plan and provide discharge instructions to the patient and the patient's caregiver as necessary when the patient is discharged home.

b. Ambulatory Surgical Center (ASC): Transfer Agreements With Hospitals

We are replacing the requirement at $\S 416.41(\mathrm{~b})(3)$, that ASCs have written transfer agreements or privileges with the local hospital with a requirement that ASCs must periodically provide the local hospital with written notice of its operation and patient population served.

c. ASC Requirements for

Comprehensive Medical History and

Physical Assessment

We are removing the current requirements at $\S 416.52$ (a) for a History and Physical within 30 days of the procedure and replacing them with requirements that defer, to a certain extent, to the ASC policy and operating physician's clinical judgment to ensure that patients receive the appropriate pre-surgical assessments tailored to the patient and the type of surgery being performed. We still require the operating physician to document any pre-existing medical conditions and appropriate test results, in the medical record, before, during and after surgery. 
In addition, we have retained the requirement that all pre-surgical assessments include documentation regarding any allergies to drugs and biologicals, and that the medical history and physical examination (H\&P), if completed, be placed in the patient's medical record prior to the surgical procedure.

d. Hospice Requirements for Medication Management

We are removing the procedural requirements at $\S 418.106$ (a)(1), related to having on the hospice staff, an individual with specialty knowledge of hospice medications.

e. Hospice Requirements: Orientation of Skilled Nursing Facility (SNF) and Intermediate Care Facilities for Individuals With Intellectual

Disabilities (ICF/IID) Staff

We are revising the requirements at $\S 418.112$ (f) to explicitly require hospices to coordinate with SNFs/NFs and ICFs/IID for assuring orientation of facility staff.

f. Hospital Quality Assessment and Performance Improvement Program (QAPI Program)

We are finalizing a new standard at 42 CFR 482.21(f), "Unified and integrated QAPI program for multi-hospital systems." For a hospital that is part of a hospital system, consisting of multiple separately certified hospitals using a system governing body that is legally responsible for the conduct of two or more hospitals, the system governing body can elect to have a unified and integrated QAPI program for all of its member hospitals if the arrangement is in accordance with all applicable State and local laws. The system governing body is responsible and accountable for ensuring that each of its separately certified hospitals meets all of the requirements of this section.

g. Hospital Requirements for Comprehensive Medical History and Physical Examinations ( $§ 482.22$, 482.24, and 482.51)

We are allowing hospitals the flexibility to establish a medical staff policy describing the circumstances under which such hospitals can utilize a pre-surgery/pre-procedure assessment for an outpatient, instead of a comprehensive medical history and physical examination (H\&P). We believe that allowing this option will greatly reduce the burden on the hospital, the practitioner, and the patient. In order to exercise this option, a hospital must document the assessment in a patient's medical record. The hospital's policy must consider patient age, diagnoses, the type and number of surgeries and procedures scheduled to be performed, comorbidities, and the level of anesthesia required for the surgery or procedure; nationally recognized guidelines and standards of practice for assessment of specific types of patients prior to specific outpatient surgeries and procedures; and applicable state and local health and safety laws.

\section{h. Hospital Infection Control Program}

We are broadly revising $\S 482.42$, and issuing a new standard at $\S 482.42(\mathrm{~d})$, "Unified and integrated infection prevention and control and antibiotic stewardship programs for multi-hospital systems." Like the requirement for a unified and integrated QAPI program, the standard for infection control permits a hospital that is part of a hospital system consisting of multiple separately certified hospitals using a single governing body, such body can elect to have a unified and integrated infection prevention and control program for all of its member hospitals in accordance with all applicable State and local laws. The system governing body is responsible and accountable for ensuring that each of its separately certified hospitals meets all of the requirements of this section. Each separately certified hospital within the system must demonstrate that-the unified and integrated infection control program is established in a manner that takes into account each member hospital's unique circumstances and any significant differences in patient populations and services offered in each hospital; the unified and integrated infection control program establishes and implements policies and procedures to ensure that the needs and concerns of each of its separately certified hospitals, regardless of practice or location, are given due consideration, and that the unified and integrated infection control program has mechanisms in place to ensure that issues localized to particular hospitals are duly considered and addressed; and a qualified individual (or individuals) has been designated at the hospital as responsible for communicating with the unified infection control program and for implementing and maintaining the policies and procedures governing infection control as directed by the unified infection control program.

i. Special Requirements for Psychiatric Hospitals

At $\S 482.61(d)$, we are clarifying the scope of authority for non-physician practitioners or Doctors of Medicine and Doctors of Osteopathic Medicine (MD/
DOs) to document progress notes of patients receiving services in psychiatric hospitals.

j. Special Requirement for Transplant Centers and Definitions

We are making a nomenclature change at 42 CFR part 482 and the transplant center regulations at $\S \S 482.68,482.70,482.72$ through 482.104 , and at $\S 488.61$. This change updates the terminology used in the regulations to conform to the terminology that is widely used and understood within the transplant community, thereby reducing provider confusion.

k. Data Submission, Clinical Experience, and Outcome Requirements for ReApproval of Transplant Centers

We are removing the requirements at $\S 482.82$ that state that transplant centers must meet all data submission, clinical experience, and outcome requirements in order to obtain Medicare re-approval. Transplant centers will still be required to comply with the CoPs at $\S \S 482.72$ through 482.104 and the data submission, clinical experience, and outcome requirements for initial Medicare approval under $§ 482.80$.

1. Special Procedures for Approval and Re-Approval of Organ Transplant Centers

We are revising $\S 488.61(\mathrm{f})$ through (h) to remove the requirements with respect to the re-approval process for transplant centers. This change corresponds to the proposed removal of the provisions at $\S 482.82$. We are retaining the requirements in $\S 488.61$ (f) through (h) that pertain to the initial approval process for transplant centers.

m. Home Health Agency (HHA)

Requirements for Verbal Notification of Patient Rights and Responsibilities

We are removing the requirements for verbal (meaning spoken) notification of all patient rights at $\S 484.50$ (a)(3), and replacing it with a requirement that verbal notice must be provided for those rights related to payments made by Medicare, Medicaid, and other federally funded programs, and potential patient financial liabilities as specified in the Social Security Act (the Act).

n. Personnel Requirements for Portable $\mathrm{X}$-Ray Technologists

We are revising $§ 486.104(a)$, "Condition for coverage: Qualifications, orientation and health of technical personnel", to focus on the qualifications of the individual performing services. 
o. Portable X-Ray Requirements for Orders

We are revising the requirements for portable x-ray orders at $\$ 486.106(a)(2)$ by removing the requirement that physician or non-physician practitioner's orders for portable x-ray services must be written and signed and replacing the specific requirements related to the content of each portable x-ray order with a cross-reference to the requirements at $42 \mathrm{CFR} 410.32$, which also apply to portable x-ray services.

p. Emergency Preparedness

Requirements: Requirements for Emergency Plans

We are removing the requirements from our emergency preparedness rules for Medicare and Medicaid providers and suppliers that facilities document efforts to contact local, tribal, regional, State, and Federal emergency preparedness officials, and that facilities document their participation in collaborative and cooperative planning efforts.

q. Emergency Preparedness

Requirements: Requirements for Annual Review of Emergency Program

We are revising this requirement so that applicable providers and suppliers review their Emergency program biennially, except for Long Term Care facilities, which will still be required to review their emergency program annually.

r. Emergency Preparedness

Requirements: Requirements for Training

We are revising the requirement that facilities develop and maintain a training program based on the facility's emergency plan annually by requiring facilities to provide training biennially (every 2 years) after facilities conduct initial training for their emergency program, except for long term care facilities which will still be required to provide training annually. In addition, we are requiring additional training when the emergency plan is significantly updated.

s. Emergency Preparedness

Requirements: Requirements for Testing

For inpatient providers, we are expanding the types of acceptable testing exercises that may be conducted. For outpatient providers, we are revising the requirement such that only one testing exercise is required annually, which may be either one community-based full-scale exercise, if available, or an individual facility-based functional exercise, every other year and in the opposite years, these providers may choose the testing exercise of their choice.

2. Proposals That Reduce the Frequency of Activities and Revise Timelines

a. Comprehensive Outpatient

Rehabilitation Facility (CORF)

Utilization Review Plans

We are amending the utilization review plan requirements at $\S 485.66$ to reduce the frequency of utilization reviews from quarterly to annually. This would allow an entire year to collect and analyze data to inform changes to the facility and the services provided.

b. CAH Annual Review of Policies and Procedures

We are changing the requirement at $\S 485.635$ (a)(4) to require a CAH's professional personnel to, at a minimum, conduct a biennial review of its policies and procedures instead of an annual review.

c. Community Mental Health Center (CMHC) Requirements for Updating the Client Assessment

At $\S 485.914$, we are removing the requirement that all CMHC clients receive an updated assessment every 30 days. Instead, we would require updates of the patient assessment in accordance with client needs and standards of practice. For clients receiving partial hospitalization services, we are retaining the 30-day assessment update time frame to be consistent with existing Medicare payment requirements for recertification of partial hospitalization services.

d. Rural Health Clinic (RHC) and Federally Qualified Health Center (FQHC) Review of Patient Care Policies

We are revising the requirement at $\S 491.9(\mathrm{~b})(4)$ that RHC and FQHC patient care policies be reviewed at least annually by a group of professional personnel, to review every other year in order to reduce the frequency of policy reviews.

\section{e. RHC and FQHC Program Evaluation}

We are revising the requirement at $\S 491.11$ (a) by changing the frequency of the required $\mathrm{RHC}$ or FQHC evaluation from annually to every other year.

3. Proposals That Are Obsolete, Duplicative, or That Contain Unnecessary Requirements

a. Hospice Aide Training and

Competency Requirements

We are revising $\S 418.76(a)(1)$ (iv) to remove the requirement that a State licensure program meet the specific training and competency requirements set forth in $\S 418.76$ (b) and (c) in order for such licensure to qualify a hospice aide to work at a Medicare-participating hospice, deferring to State licensure requirements.

b. Medical Staff: Autopsies

We are finalizing our proposal to remove the requirement for hospitals at $\S 482.22(\mathrm{~d})$, which states that a hospital's medical staff should attempt to secure autopsies in all cases of unusual deaths and of medical-legal and educational interest. We are deferring to State law regarding such medical-legal requirements.

\section{c. Hospital and CAH Swing-Bed Requirements}

We are removing the cross reference to $\S 483.10(\mathrm{f})(9)$ at $\S 482.58(\mathrm{~b})(1)$ (for hospital swing-bed providers) and $\S 485.645$ (d)(1) (for CAH swing-bed providers); the repealed provisions gave a resident the right to choose to, or refuse to, perform services for the facility if they so choose.

We are removing the cross-reference to $\S 483.24$ (c) at $\S 482.58(\mathrm{~b})(4)$ (for hospital swing-bed providers) and $\S 485.645(\mathrm{~d})$ (4) (for CAH swing-bed providers) requiring that the facility provide an ongoing activity program based on the resident's comprehensive assessment and care plan directed by a type of qualified professional specified in the regulation.

We are removing the cross-reference to $\S 483.70(\mathrm{p})$ at $\S 482.58(\mathrm{~b})(5)$ (for hospital swing-bed providers) and $\S 485.645$ (d)(5) (for CAH swing-bed providers requiring facilities with more than 120 beds to employ a social worker on full-time basis).

We are removing the cross-reference to $\S 483.55(\mathrm{a})(1)$ at $\S 482.58(\mathrm{~b})(8)$ (for hospital swing-bed providers) and $\S 485.645(\mathrm{~d})(8)$ (for CAH swing-bed providers) requiring that the facility assist residents in obtaining routine and 24-hour emergency dental care.

d. Home Health Agency Home Health Aide Requirements

We are revising $\S 484.80$ (c)(1) to clarify that skill competencies may be assessed by observing an aide performing the skill with either a patient or a pseudo-patient as part of a simulation. We are defining the terms "pseudo-patient" and "simulation" in $\S 484.2$.

We are revising the requirement at $\S 484.80(\mathrm{~h})$ related to completing a full competency evaluation when an aide is found to be deficient in one or more skills. Instead of completing a full competency evaluation, an aide would only be required to complete retraining 
and a competency evaluation directly related to the deficient skills.

\section{e. CAH Disclosure Requirements}

We are removing $\S 485.627(\mathrm{~b})(1)$, the requirement for CAHs to disclose the names of people with a financial interest in the CAH. This is currently a requirement under the program integrity requirements at $42 \mathrm{CFR} 420.206$, which are referenced in the provider agreement rules in 42 CFR 489.53(a)(8), making this CAH CoP requirement duplicative of those regulations.
3. Summary of Costs and Benefits for Regulatory Provisions To Promote Efficiency, Transparency, and Burden Reduction

\section{Overall Impact}

This final rule will create savings and reduce burden in many areas. Several of the changes will create measurable monetary savings for providers and suppliers, while others will create less quantifiable savings of time and administrative burden. We anticipate a total first year net savings of approximately \$843 million, and slightly more in future years.

2. Section-by-Section Economic Impact Estimates

Table 1 summarizes the provisions for which we are able to provide specific estimates for savings or burden reductions (these estimates are uncertain and could be substantially higher or lower, as explained in the regulatory impact analysis section of this final rule):

Table 1-Summary of Net Savings by Provision

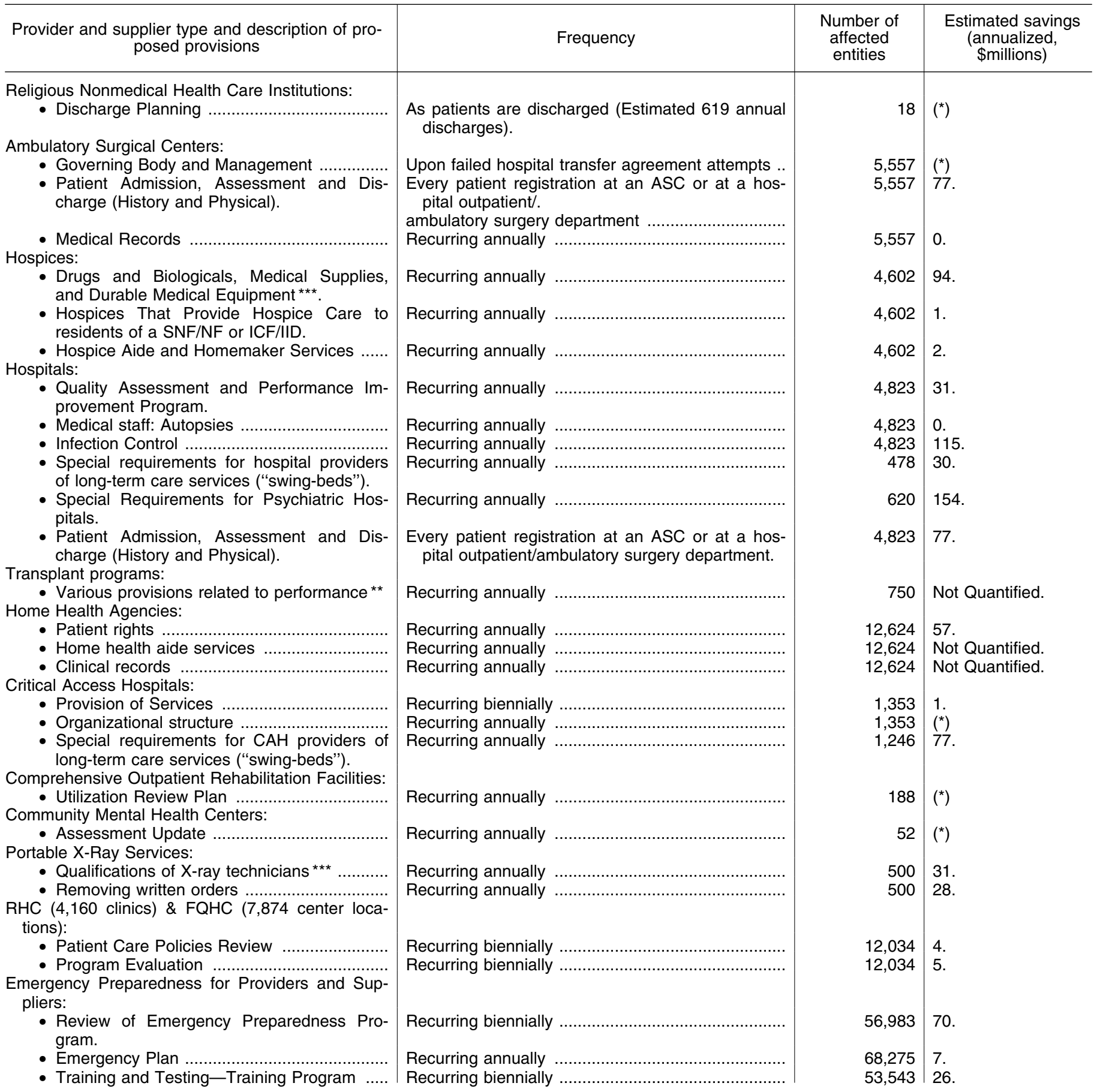


TABLE 1-Summary of Net SAVINGs By Provision-Continued

\begin{tabular}{|c|c|c|c|}
\hline $\begin{array}{l}\text { Provider and supplier type and description of pro- } \\
\text { posed provisions }\end{array}$ & Frequency & $\begin{array}{l}\text { Number of } \\
\text { affected } \\
\text { entities }\end{array}$ & $\begin{array}{l}\text { Estimated savings } \\
\text { (annualized, } \\
\text { \$millions) }\end{array}$ \\
\hline - Training and Testing-Testing & Recurring annually & 36,971 & 21. \\
\hline
\end{tabular}

${ }^{*}$ Amount is less than half a million dollars and rounds to zero.

** These include changes to the following requirements: Special Requirements for Transplant Programs; Data submission, Clinical Experience, and Outcome Requirement for Re-approval of Transplant Programs; and Special Procedures for Approval and Re-Approval of Organ Transplant Programs.

${ }_{* \star \star}$ This estimate is for first full year savings only and will increase in future years.

B. Provisions of the Proposed Rule and Analysis and Response to Public Comments on "Medicare and Medicaid Programs; Regulatory Provisions To Promote Program Efficiency, Transparency, and Burden Reduction"

In response to our September 20, 2018 proposed rule (83 FR 47686), we received 620 public comments. Commenters included individuals, healthcare professionals and corporations, national associations, health departments and emergency management professionals, and individual facilities that would be impacted by the regulation. Generally, the comments received were supportive. Most comments were centered around the proposed revisions to the emergency preparedness regulations for Medicare and Medicaid providers and suppliers. We have organized our responses to the comments as follows: (1) Comments specific to individual types of providers and suppliers (2); general comments; and (3) comments regarding our savings estimates.

1. Religious Nonmedical Health Care Institutions (RNHCIs)—Discharge Planning (§ 403.736(a) and (b))

Section 1861(ss)(1) of the Act defines the term "Religious Nonmedical Health Care Institution"' (RNHCI) and lists the requirements that a RNHCI must meet to be eligible for Medicare participation.

Section 403.736(a) and (b) of the RNHCI's CoPs, as amended in the November 28, 2003 Federal Register (68 FR 66710), requires RNHCIs to have a discharge planning process for patients.

Since the RNHCI's religious tenets prohibit conventional or unconventional medical treatment of a beneficiary, and medical postinstitution services are not utilized by RNHCI patients, we believe that extensive discharge requirements are unnecessarily burdensome. Therefore, we proposed a more condensed and flexible process for discharge planning and instructions for RNHCIs. We proposed to remove the requirements at $\S 403.736$ (a) and (b), and proposed instead to require RNHCIs to provide discharge instructions to the patient or the patient's caregiver when the patient is discharged home.

The majority of commenters expressed strong support for the proposed changes to the RNHCIs discharge planning process. We did not receive any comments in opposition to the proposed requirement; therefore, we are incorporating the changes as proposed in this final rule.

Comment: One commenter stated that they agreed with allowing flexibility and giving the institution the freedom to determine which patients should be provided a discharge plan. However, they commented that there should be a way to monitor this process to make institutions accountable and not omit providing a discharge plan if a patient needs one.

Response: As for all providers and suppliers, Medicare surveys RNHCIs for compliance with the CoPs. We believe this enforcement process adequately ensures that RNHCIs are correctly interpreting and following our requirements.

Comment: The majority of the commenters stated that they agree with the changes proposed to the discharge planning process at $\S 403.736$ (a) and (b). They stated that this change would reduce burden and allow greater flexibility to the RNHCIs.

Response: We appreciate the comments received on the proposed changes for RNHCIs and will finalize the changes as proposed.

Final Rule Action: We are finalizing the proposed changes without changes. Contact: Mary Collins, (410) 7863189.

\section{Ambulatory Surgical Centers}

Section 416.2 of our rules defines an ambulatory surgical center (ASC) as any distinct entity that operates exclusively for the purpose of providing surgical services to patients not requiring hospitalization, in which the expected duration of services would not exceed 24 hours following an admission. The surgical services performed at ASCs are scheduled, primarily elective, non-lifethreatening procedures that can be safely performed in an ambulatory setting. We received 99 timely public comments on our proposed changes to the ASC CfC requirements. Commenters included ASC industry associations, healthcare systems, national accreditation organizations, clinician associations, individual ASCs, and clinicians. Overall, the majority of the commenters were supportive of the goals of the proposed changes.

Summaries of the major issues and our responses are set forth below.

a. Governing Body and Management; Hospitalization Requirements (§ 416.41(b)(3)(i) and (ii))

We proposed to remove the requirement for a written hospital transfer agreement or hospital physician admitting privileges at $\S 416.41(\mathrm{~b})(3)$. The requirements in $\S 416.41(\mathrm{~b})(1)$ and (2) continue to require the ASC to have an effective procedure for the immediate transfer, to a hospital, of patients requiring emergency medical care beyond the capabilities of the ASC and that the hospital must be a local hospital that meets the requirements for payment for emergency services under $\S 482.2$. As part of this revision, ASCs are not precluded from obtaining hospital transfer agreements or hospital physician admitting privileges when possible.

Comment: The comments submitted regarding the removal of the transfer agreement were almost evenly split between supporters and opponents. Specifically, the ASCs supported the removal of the transfer agreement and hospitals were opposed to the removal of the transfer agreement. Comments in support of removal of the written hospital transfer agreement stated that the current requirement is unnecessary, obsolete and extremely burdensome because of the small number of patient transfers, the creation of The Emergency Medical Treatment and Labor Act (EMTALA), and the exhaustive administrative paperwork and negotiation burden that is required when the local hospital system refuses to sign the written hospital transfer agreement. Comments in support of the 
removal also stated that ASCs should not be forced to close their businesses because regulations cannot be met due to competition issues with the local hospital and their outpatient surgery center. Comments opposing removal of the written hospital transfer agreement stated that transfer agreements have the potential to ensure that there is a plan for emergencies, that appropriate continued care will be delivered, and that both the ASC and hospital communicate with one another. In addition, we received several comments that suggested the regulation should instead specify that the ASC would be deemed to have met the hospital transfer agreement provision if a "good faith effort" was documented. One commenter suggested that instead of an all or nothing provision, ASCs should periodically provide local hospitals with a written notice. The commenter contended that this requirement would notify the hospital of ASC services in the community and the types of patients that are receiving care that may need additional care beyond the capability of the ASC.

Response: We continue to believe that, because of the existing EMTALA regulations, the small number of transfers, and the burden ASCs incur when faced with local hospital competition issues, removing this requirement is necessary and appropriate. We agree that communication between ASCs and hospitals is important; however, we do not agree that a mandated transfer agreement is a necessary or effective method to assure this communication. In response to the commenter's suggestions described above, and to assure that hospitals are aware of the potential for receiving patient transfers from an ASC, we are revising our proposal at $\S 416.41(\mathrm{~b})(3)$ to require the ASC to periodically provide the local hospital with written notice of its operation and patient population served. For example, the notice would include details such as hours of operation and the procedures that are performed in the ASC. Providing written notice, rather than securing a transfer agreement, will alleviate the administrative burden of negotiating or being denied negotiating opportunities associated with the requirement of a written transfer agreement between the ASC and hospital. We are requiring that the notice be provided "periodically" to the local hospital to ensure the ASC keeps the local hospital informed and up-to-date on ASC information and any patient population changes. The "periodically" phrasing is similar to the reappraisal requirement for the medical staff privileges in ASCs located at § 416.45(b), "Medical staff- Standard: Reappraisals", and is meant to have the same meaning. This change does not preclude those ASCs and hospitals with functional working relationships to continue to have written transfer agreements, which we encourage, and prior preparations in place for patient transfers in the event of an emergency.

b. Patient Admission, Assessment and Discharge (§ 416.52(a)(1), (2), (3) and (4))

The current regulations at $\S 416.52$ require ASCs to ensure that a physician or other qualified practitioner provide a comprehensive medical history and physical assessment (H\&P) completed not more than 30 days before the date of the scheduled surgery.

We proposed to remove the current requirements at $\S 416.52$ (a) and replace them with requirements under the facility's established policies for presurgical medical histories and physical examinations (H\&P), including any associated testing, and the operating physician's clinical judgment, to ensure patients receive the appropriate presurgical assessments that are tailored for the patient and the type of surgery being performed. We proposed to require each ASC to establish and implement a policy that identifies patients who require an $\mathrm{H} \& \mathrm{P}$ prior to surgery. We proposed that the policy would include the time frame for the $\mathrm{H} \& \mathrm{P}$ to be completed prior to surgery. We proposed that the policy would be required to consider the age of patients, their diagnoses, the type and number of surgeries that are scheduled to be performed at one time, all known comorbidities, and the planned level of anesthesia for the surgery to be performed. ASCs would not be limited to these factors, and would be permitted to include others to meet the needs of their patient populations. Furthermore, we proposed that each ASC's policy would be required to follow nationally recognized standards of practice and guidelines, as well as applicable state and local health and safety laws. To conform to the proposed changes to the medical history and physical examination requirements at $\S 416.52$ (a), we proposed to revise the requirement at $\S 416.47(\mathrm{~b})(2)$, that states "Significant medical history and results of physical examination," by adding “as applicable.",

Comment: A majority of commenters supported the proposed change to remove the medical H\&P examination requirement no more than 30 days before the date of the scheduled surgery, and defer to the ASCs established policies for pre-surgical H\&Ps and the operating physician's clinical judgment. The comments agreed that allowing ASCs flexibility to establish patient policies, and encouraging the use of clinician judgment, are appropriate to assure patient health and safety while also reducing the burden on patients and providers, and reducing expenditures on potentially unnecessary pre-operative testing that is performed because it is required by policy. However, a small number of comments supported only part of this change, suggesting instead that CMS should retain the $\mathrm{H} \& \mathrm{P}$ requirement while allowing the ASC the discretion to determine the timeframe for the H\&P relative to the date of surgery. Another commenter opposed any changes and recommended retaining the H\&P requirement and 30-day time frame. One commenter stated that they believe the burden of assessing patients prior to surgery would be shifted from one provider (the primary care physician) to another (the anesthesiologist), and that allowing ASCs the flexibility to establish their own policies based on their clinical judgment and patient population needs would burden ASCs and healthcare workers, create inefficiencies, and lead to variations in standards of care from facility to facility.

Response: We appreciate the support of the vast majority of commenters, and continue to believe that the change is appropriate to support patient health and safety while eliminating the burdens of potentially unnecessary examinations and tests. The content of ASC-wide policies surrounding the appropriate use of medical histories and physicals, as well as pre-operative testing, could be informed by specialty societies, medical literature, past experience, or other factors. We disagree that variations between facilities would be an inherently undesirable effect of the change; variations to take into account unique patient needs and facility characteristics are preferable to a "one size fits all" approach of mandatory 30 day H\&Ps. Allowing ASCs and physicians to work together to implement their own policies, based on their clinical judgment and patient population served, will provide the most optimal balance between burden and necessary examinations and testing, by identifying when a medical H\&P examination should be completed, if appropriate. We are finalizing the proposal to require ASCs to address certain patient characteristics, such as age, diagnosis, the type and number of procedures, comorbidities and the planned anesthesia level, when 
developing their policies and procedures for pre-surgical examinations and testing. We believe this change will ensure those patients who would actually be protected by a medical H\&P examination will receive one based on ASC policy, and in a time frame established by that policy, thereby reducing burden on physicians, facilities, and patients. We reiterate that ASCs are still required to perform a patient assessment upon admission before surgery, that the ASC is not precluded from retaining the $\mathrm{H} \& \mathrm{P}$ requirement in facility policies, and that we are not discouraging pre-surgical H\&Ps where clinically indicated.

Comment: One commenter expressed concern over the wording of the proposed regulation text in $\S 416.52(\mathrm{a})(1)(\mathrm{iii})$, stating that the ASC policy must follow nationally recognized standards of practice and guidelines. The commenter believed the term "follow" could be problematic for ASCs, and be interpreted as being required to "adhere" to national guidelines that are not delineated, thus depriving the ASC of the ability to determine what clinical practices make the best sense for its patients.

Response: We agree and have revised the regulation text to be consistent with the regulation text that is being finalized for hospital outpatient H\&P requirements. We are finalizing the regulation text to state that the ASC policy must be based on any applicable nationally recognized standards of practice and guidelines, and any applicable State and local health and safety laws.

Comment: One commenter asked CMS to coordinate any changes to the regulations with updates to the accreditation organizations (AOs) standards.

Response: National accreditation organizations must meet or exceed CMS standards, and their standards must be updated, as appropriate, to reflect changes in the CMS regulations. As AOs may choose to exceed CMS requirements, so they may choose to retain any or all of the requirements that we are removing in this final rule to the extent that they do not conflict with any of our revisions.

We did not receive any public comments on the proposed technical change to the medical records proposed at $\S 416.47(\mathrm{~b})(2)$ and are finalizing the technical change to the medical records section as proposed.

Final Rule Action:

1. Rather than deleting, we are finalizing revisions to $\S 416.41(\mathrm{~b})(3)$ to require ASCs to periodically provide the local hospital with written notice of its operation and patient population served.

2 . We are finalizing the proposal to revise the requirement at $\S 416.47(\mathrm{~b})(2)$, to state "Significant medical history and results of physical examination, as applicable."

3. We are finalizing the proposal to eliminate the requirement at $\$ 416.52$ (a) for each patient to have a medical history and physical assessment completed by a physician not more than 30 days before the scheduled surgery, and replace it with the requirement for ASCs to develop and maintain a policy that identifies those patients who require a medical history and physical examination prior to surgery. In addition, require the policy to include the timeframe for the medical history and physical examination to be completed prior to surgery. The policy must also address, but not be limited to, the following factors: Patient age, diagnosis, the type and number of procedures scheduled to be performed on the same surgery date, known comorbidities, and the planned anesthesia level. Upon admission, each patient must have a pre-surgical assessment completed by a physician or other qualified practitioner in accordance with applicable State health and safety laws, who will be performing the surgery.

4. We are revising $\S 416.52(a)(1)(i i i)$ to clarify that the ASC policy must be based on nationally recognized standards of practice and guidelines, and applicable State and local health and safety laws.

Contact: CAPT Jacqueline Leach, USPHS, 410-786-4282.

\section{Hospice}

a. Hospice Aide and Homemaker Services (§ 418.76)

We proposed to revise $\S 418.76$ (a)(1)(iv) to remove the requirement that a hospice aide training State licensure program must meet the specific training and competency requirements set forth in $\S 418.76$ (b) and (c) in order to be deemed an appropriate qualification for employment. This change would defer to State licensure requirements, except in states where no requirements exist.

Comment: Many comments supported the proposed revision to defer to existing state requirements for hospice aide training, and only impose Federal requirements in the absence of state standards. However, other comments did not support this proposed change, arguing that state education and training standards for hospice aides should not be accepted as being sufficient to assure patient health and safety.
Response: Deference to stateestablished standards regarding the training and competency of health care professionals is standard practice. States already establish such standards for health care professions such as nursing, laboratory technicians, phlebotomists, and therapists, to name a few. Seventysix percent of states have already established their own qualification standards for aides, aides furnishing services in those states are already permitted to provide services to individuals through private pay agencies without meeting the Medicare standards, and there is no indication that these already applicable standards are insufficient to assure patient health and safety. As deference to state standards is the norm across the health care spectrum, and as current state standards are already demonstrated to be sufficient to assure patient health and safety, we see no reason to impose a separate Federal standard. Therefore, we are finalizing this proposed change. In the absence of state requirements, hospices will continue to be required to assure that an aide meets the Federal training standards. Furthermore, all hospices in all states will continue to be required to comply with the existing requirements that hospice aides may only perform those skills that are consistent with the training that the aide has received (§ $418.76(\mathrm{~g})(2)(\mathrm{iv})$ ), and that, if an area of concern is verified by the hospice during an on-site aide supervision visit, then the hospice must conduct, and the hospice aide must complete, a competency evaluation in accordance with $\S 418.76$ (c) and (h)(1)(iii).

b. Drugs and Biologicals, Medical Supplies, and Durable Medical Equipment (§ 418.106(a)(1) and (e)(2)(i))

We proposed to delete the requirements at $\S 418.106$ (a)(1), which required hospices to ensure that the interdisciplinary group confers with an individual with education and training in drug management as defined in hospice policies and procedures and State law, who is an employee of or under contract with the hospice, to ensure that drugs and biologicals meet each patient's needs. Meeting each patient's needs would continue to be the responsibility of all Medicareparticipating hospices in accordance with the requirements of all other hospice CoPs.

Comment: We received numerous comments that both supported and opposed the proposal to eliminate the process requirement that a hospice must confer with an individual with expertise in medication management regarding 
the plan of care for each patient. Many commenters agreed that this process requirement is no longer necessary because this is standard practice in hospices. However, other commenters, while agreeing that it is standard practice, still believe that there is value in having a distinct regulatory requirement to this effect, due to the important role that medications play in hospice care and the potential for safety lapses.

Response: Hospices would continue to be required to comprehensively assess patients on a regular schedule and on an as needed basis in accordance with the requirements of $\S 418.54$ (a), (b) and (d), and to assure that each patient's plan of care is developed and continually updated to meet each patient's needs as identified in the assessment process in accordance with the requirements of 418.56 (b) through (d). These existing regulations, which we are not revising, focus on assuring the outcomes of safe, effective, patientcentered care. Furthermore, hospices will still be required to comply with the quality assessment and performance improvement (QAPI) CoP at $§ 418.58$, which requires hospices to monitor patient outcomes and implement improvement projects to address identified areas of concern. To the extent that patient outcomes are not being achieved due to problems with medication management, both the hospice's internal QAPI program and the external hospice survey process will be capable of identifying and addressing those problems, regardless of the removal of this process requirement. In light of these existing requirements, and in response to the support for the proposed change expressed by a variety of commenters, we are finalizing the proposed change to remove the process requirement at §418.106(a)(1) without revisions.

We proposed to replace the requirement at $\S 418.106(\mathrm{e})(2)$ that hospices provide a physical paper copy of policies and procedures, which are written to guide the actions of hospice staff, with a requirement that hospices provide information regarding the use, storage, and disposal of controlled drugs to the patient or patient representative, and family, which can be developed in a manner that speaks to the perspectives and information needs of patients, families, and caregivers. This information would be provided in a more user-friendly manner, as decided by each hospice. Hospices would be free to choose the content and format(s) that best suits their needs and the needs of their patient population. We proposed to require that, regardless of the format chosen, this information would have to be provided to patients, families and caregivers in a manner that allowed for access to the information on a continual, as-needed basis.

We would continue to require that hospices discuss the information regarding the safe use, storage and disposal of controlled drugs with the patient or representative, and the family/caregiver(s), in a language and manner that they understand to ensure that these parties are effectively educated. This requirement is included in the current hospice CoPs and is consistent with Department of Health and Human Services guidance regarding Title VI of the Civil Rights Act ("Guidance to Federal Assistance Recipients Regarding Title VI Prohibition Against National Origin Discrimination Affecting Limited English Proficient Persons," 68 FR 47311, August 8, 2003, https:// www.hhs.gov/civil-rights/forindividuals/special-topics/limitedenglish-proficiency/guidance-Federalfinancial-assistance-recipients-title-VI/). We continue to expect hospices to utilize technology, such as telephonic interpreting services and any other available resources for oral communication in the individual's primary or preferred language. We would also continue to require that hospices document in the patient's clinical record that the information was provided and discussed.

Comment: We received many comments regarding the proposed change to allow hospices to determine the content and form of the controlled drug storage, use, and disposal notice for patients and families. Commenters universally supported the goal of improving patient and family education on this subject and supported the shift away from providing policies and procedures. However, a few commenters raised concern about the intersection of this change with section 3222 of the recently adopted Substance UseDisorder Prevention that Promotes Opioid Recovery and Treatment for Patients and Communities Act (“SUPPORT Act”) (Pub. L. 115-271), that requires hospices, which permit their employees to dispose of medications in the patient's home, to provide their written policies and procedures to patients, families and caregivers. This provision, which amends section 302 of the Controlled Substances Act (21 U.S.C. 822), is under the jurisdiction of the Department of Justice.

While most commenters expressed appreciation for the proposal to allow hospices to determine the form and content of the notice, other commenters suggested that CMS should develop education materials that hospices must provide to patients and families. Whether hospice or CMS-generated, commenters suggested that using alternative formats such as pictorial infographics and videos may be valuable tools in communicating this important information. Commenters also suggested that the information should be accessible to all individuals, regardless of impairments or language spoken.

Response: In light of the changes included in section 3222 of the SUPPORT Act, it is not appropriate to finalize this proposed change. However, we encourage hospices to develop easily understood materials that explain safe storage, use, and disposal of controlled drugs to patients, their families, and caregivers in addition to meeting the regulatory requirement to provide a copy of the hospice's clinical policies and procedures. We continue to believe that providing such materials is a positive practice for improving comprehension of this crucial information and improving compliance with safe handling, use, and disposal practices.

c. Hospices That Provide Hospice Care to Residents of a SNF/NF or ICF/IID (§ 418.112(c)(10) and (f))

Section 418.112(f) of the hospice CoPs, as finalized in the 2008 Hospice CoP final rule (73 FR 32088), requires hospices to assure orientation of Skilled Nursing Facility/Nursing Facility (SNF/ $\mathrm{NF}$ ) or ICF/IID staff furnishing care to hospice patients. We proposed to remove $\S 418.112$ (f) and add a new requirement at $\S 418.112$ (c)(10), "Written agreement," to permit both entities to negotiate the mechanism and schedule for assuring orientation of facility staff.

Comment: While comments supported the intent behind the proposed change to permit hospices and long term care facilities to negotiate the roles and responsibilities for orienting long term care facility staff to the hospice philosophy of care and hospice procedures, some comments did not support moving the topic into the content of the written agreement. Comments stated that requiring this subject to be addressed in the written agreement would create a onetime burden for hospices of renegotiating the written agreement with each long term care facility, and that this burden was not acceptable even in light of the potential long-term regulatory relief of the proposed change. Some comments suggested that the current regulations at 
$\S 418.112(\mathrm{f})$ instead be revised to allow for hospices and facilities to negotiate their respective roles and

responsibilities outside of the written agreement.

Response: We agree with commenters that the goal of regulatory flexibility is worthwhile, and we appreciate the feedback regarding the scope of the regulatory burden that would be incurred when renegotiating existing contracts with long term care facilities. In light of the burden concerns raised in the comments, we agree that a different approach to achieving the same goal is warranted. We are not finalizing the proposal to move the requirements related to facility staff orientation and training from a standalone requirement to a provision in the written agreement. In order to achieve the original regulatory goal of adding flexibility and reducing hospice costs for this activity, we are revising existing $\S 418.112$ (f), Orientation and training of staff, to clarify that a hospice must consult with and thus share responsibility with the facility to assure facility staff orientation and training. We received 26 timely public comments pertaining to all proposed changes to the hospice requirements. Commenters included hospice industry associations, individual hospice providers, national accrediting organizations, clinician associations, and consumer advocacy groups. Overall, the majority of commenters were supportive of the goal of the proposed changes. Comments not directly related to our proposals are summarized below.

Comment: A few comments specifically related to the hospice CoPs were submitted in response to the solicitation for ideas for further burden reduction efforts. Comments included removing the core services requirement for dietary counseling and providing waivers for social worker supervision.

Response: We appreciate the suggestions, and will consider revising the social work supervision requirements in future rulemaking. The hospice interpretive guidelines related to $\S 418.114(\mathrm{~b})(3)$ (State Operations Manual, Pub. 100-07, Appendix M, https://www.cms.gov/Regulations-andGuidance/Guidance/Manuals/ downloads/som107ap_m_hospice.pdf, accessed on January 16, 2019) state, "Each hospice must employ or contract with at least one MSW to serve in the supervisor role. . . ."We believe that this existing flexibility regarding contracting for this service, when coupled with the fact that the supervision role can be performed remotely, is adequate to address concerns regarding the provision of social work supervision at this time while we consider this waiver suggestion. Dietary counseling as a core service is a statutory requirement (see section 1861(dd)(2)(A)(ii)(I) of the Act)) and cannot be repealed through regulatory mechanisms.

Comment: We received numerous comments with suggestions related to Medicare payment requirements for hospice services (for example, notice of election requirements and the coverage requirements for continuous home care level of care), use of the CMS Common Working File, hospice quality measures, Medicaid payment issues, and Medicare audits.

Response: These comments are not within the scope of this regulation, which is related to the health and safety standards for Medicare providers. We publish an annual proposed hospice payment rule, and comments related to payment policies and rates may also be submitted to that rule for consideration. All out of scope comments have been shared with the appropriate components within CMS.

Final Rule Action:

1. We are finalizing the proposed changes to $\S \S 418.76(a)(1)$ (iv) and 418.106(a)(1) without change. We are not finalizing our proposed change to 418.106(e)(2)(i).

2 . Revise $\S 418.112(\mathrm{f})$ to require hospice and facilities to share responsibility for facility staff orientation and training.

Contact: Danielle Shearer, 410-7866617.

\section{Hospitals}

a. Quality Assessment and Performance Improvement Program (§ 482.21)

We proposed a new standard at § 482.21(f), "Unified and integrated QAPI program for multi-hospital systems." We would allow that for a hospital that is part of a hospital system consisting of two or more separately certified hospitals subject to a system governing body legally responsible for the conduct of each hospital, the system governing body could elect to have a unified and integrated QAPI program for all of its member hospitals after determining that such a decision is in accordance with all applicable State and local laws. The system governing body would be responsible and accountable for ensuring that each of its separately certified hospitals meets all of the requirements of this section. Each separately certified hospital subject to the system governing body would have to demonstrate that: The unified and integrated QAPI program was established in a manner that took into account each member hospital's unique circumstances and any significant differences in patient populations and services offered in each hospital; and the unified and integrated QAPI program establishes and implements policies and procedures to ensure that the needs and concerns of each of its separately certified hospitals, regardless of practice or location, are given due consideration, and that the unified and integrated QAPI program has mechanisms in place to ensure that issues localized to particular hospitals are duly considered and addressed.

Comment: Most commenters supported the proposal to allow hospitals that are part of a multihospital system to have a unified and integrated QAPI program. A few commenters expressed appreciation for the expanded flexibility that this proposal would afford hospitals by reducing burden, increasing efficiencies, and eliminating the duplication of efforts.

A few commenters generally supported this proposal, but recommended that individual, hospitalspecific data be recorded and made available to the system's governing body and the public. These data, the commenters stated, would help to identify best practices and processes from facilities that are excelling in certain areas and will account for and address performance outliers across the broader hospital system. Finally, another commenter expressed concern that the proposed requirement might group QAPI scores together and hide poor performance, which they stated may mislead consumers about the sitespecific care they are receiving.

Response: We thank the commenters for their support. We believe that a hospital's governing body should be afforded the option of unifying and integrating the various member hospitals within their multi-hospital system into a unified QAPI program. Such a model would incorporate each individual hospital's QAPI program, which would enable increased efficiencies, innovations, provider flexibility, and allow for the dissemination of best practices for patient care while also potentially improving patient safety and outcomes. We also believe that a unified QAPI model is a natural progression for a multi-hospital system that utilizes a system governing body (as allowed at $\S 482.12$ ) and a unified medical staff (as allowed at $\S 482.22$ ).

In response to the commenter's concerns regarding individual hospital data, we agree that hospital specific data should be used to address specific 
individual hospital issues and to identify and disseminate best practices. As we have proposed, "the system governing body is responsible and accountable for ensuring that each of its separately certified hospitals meets all of the requirements of this section." We do not see this requirement as prohibiting an individual hospital from reporting its own data to the governing body and most especially to the unified and integrated QAPI program, since we are requiring that each separately certified hospital in the system demonstrate that the unified and integrated QAPI program takes into account each member hospital's unique circumstances as well as any significant differences in patient populations and services offered in each hospital. Each hospital must also demonstrate that the unified and integrated program has mechanisms in place to ensure that issues localized to particular hospitals are duly considered and addressed.

We are unclear as to what the commenter means by "QAPI scores" and to what the commenter is referring regarding the grouping of "QAPI scores together" in order to "hide poor performance.” The current QAPI CoP does not require anything related to "QAPI scores" and we are not finalizing any such requirements in this rule. We believe that the commenter might have been confusing QAPI with the various data that are collected for the Inpatient Quality Reporting Program. These programs are unrelated and the quality reporting program remains unchanged by this rule.

Comment: One commenter recommended that CMS include the following language in proposed $\S 482.21$ (f)(2) regarding a hospital's medical staff: “. . . Including consulting with each of its separately certified hospital's medical staff." The commenter stated that a hospital's medical staff brings a unique clinical perspective to the activities of the governing body with regard to quality and safety issues. The commenter also urged CMS to clarify that the proposed requirement will not include an Ongoing Professional Practice

Evaluation and Focused Professional Practice Evaluation, which they state, is the responsibility of the hospital's medical staff.

Response: While we agree with the commenter that a hospital's organized medical staff brings a unique clinical perspective to the activities of the governing body with regard to quality and safety issues, we believe that a number of the hospital CoPs already effectively ensure that this clinical perspective is heard by the governing body while also holding the medical staff responsible and accountable for these patient safety and quality of care issues. For example, the provision at $\S 482.12(a)(1)$, under the hospital Governing body CoP, requires that the hospital's governing body must, "consult directly with the individual assigned the responsibility for the organization and conduct of the hospital's medical staff, or his or her designee. At a minimum, this direct consultation must occur periodically throughout the fiscal or calendar year and include discussion of matters related to the quality of medical care provided to patients of the hospital." This requirement applies to all hospitals, governing bodies, and medical staffs, regardless of organizational structure.

Additionally, the QAPI CoP itself, at $\S 482.21(\mathrm{e})$, contains a standard that requires the hospital medical staff (among other hospital leaders) to be responsible and accountable for ensuring that the QAPI program is focused on improved quality of care and patient safety. Similarly, the Medical staff CoP requirement at $\S 482.22$ (b) requires that the hospital's medical staff "must be well organized and accountable to the governing body for the quality of the medical care provided to patients." And finally, at $\S 482.22(\mathrm{~b})(4)(\mathrm{iii})$ and (iv), the CoPs require that a separately certified hospital, which uses a unified and integrated medical staff accountable to a system governing body, must demonstrate that its unified and integrated medical staff: (1) Is established in a manner that takes into account each member hospital's unique circumstances and any significant differences in patient populations and services offered in each hospital and (2) establishes and implements policies and procedures to ensure that the needs and concerns expressed by members of the medical staff, at each of its separately certified hospitals, regardless of practice or location, are given due consideration, and that the unified and integrated medical staff has mechanisms in place to ensure that issues localized to particular hospitals are duly considered and addressed. Therefore, we do not believe that any additional language is needed here.

Comment: One commenter requested that CMS include "affiliates" and CAHs in the unified and integrated QAPI and infection control requirements. The commenter defines "affiliates" as hospitals and providers within a healthcare system that may bill under separate Tax Identification Numbers (TINs). The commenter noted that this option would afford hospitals additional flexibility and ease administrative burden.

Response: We are not clear on whether the commenter is confusing TINs and CMS Certification Numbers (CCNs), which CMS uses to distinguish separately certified hospitals, CAHs, and other Medicare-participating providers and suppliers for survey and certification purposes in determining compliance with the CoPs and CfCs specific to each provider and supplier type. We do not use TINs in our determination of when a facility requires separate certification.

A CAH must be separately evaluated for its compliance with the CAH CoPs (found at 42 CFR part 485, subpart F), which would not include the requirements included in this section of the rule since these are hospital CoPs. It would not be possible to evaluate the CAH's compliance as part of an evaluation of a hospital's compliance. However, this does not preclude a multi-hospital system's single governing body from also serving as the CAH's governing body, so long as the governing body clearly identifies the policies and decisions that are applicable to the $\mathrm{CAH}$.

Final Rule Action: We are finalizing the requirements in $\S 482.21$ (f), without modification.

Contact: Alpha-Banu Wilson, 410786-8687.

b. Medical Staff, Medical Records

Services, and Surgical Services

(§§ 482.22, 482.24, and 482.51)

Hospital Medical History and Physical Examination Requirements

We proposed to revise the current requirements at $\S 482.22$ (c)(5)(i) and (ii) with respect to medical staff bylaws, and to allow for an exception under the proposed paragraph (c)(5)(iii). We are retaining the current language in paragraphs (c)(5)(i) and (ii) that the $\mathrm{H} \& \mathrm{P}$, and any update to it, must be completed and documented by a physician (as defined in section 1861(r) of the Act), an oral and maxillofacial surgeon, or other qualified licensed individual in accordance with State law and hospital policy. We proposed to include this same language regarding who can complete and document the assessment in the proposed provision at §482.22(c)(5)(iii). This provision would require the medical staff bylaws to state that an assessment of the patient (in lieu of the requirements of paragraphs (c)(5)(i) and (ii)) be completed and documented after registration, but prior to surgery or a procedure requiring anesthesia services, when the patient is 
receiving specific outpatient surgical or procedural services and when the medical staff has chosen to develop and maintain a policy that identifies, in accordance with the requirements at paragraph (c)(5)(v), specific patients as not requiring a comprehensive medical history and physical examination, or any update to it, prior to specific outpatient surgical or procedural services. The proposed paragraphs (c)(5)(iii) and (iv) would require the medical staff to develop and maintain a policy that identifies those patients for whom the assessment requirements of paragraph (c)(5)(iii) would apply. We also proposed a new requirement at paragraph (c)(5)(v) for a medical staff that chooses to develop and maintain a policy for the identification of specific patients to whom the assessment requirements in paragraph (c)(5)(iii) would apply. Under this proposed paragraph, if the medical staff exercised the option to perform a simplified assessment in some cases, the written policy would have to indicate the specific outpatient surgical or procedural services to which it applied. The policy for each procedure would need to indicate the hospital's consideration of patient age, diagnoses, the type and number of surgeries and procedures scheduled to be performed, comorbidities, and the level of anesthesia required for the surgery or procedure; nationally recognized guidelines and standards of practice for assessment of specific types of patients prior to specific outpatient surgeries and procedures; and applicable State and local health and safety laws.

In order to make clear that this proposed requirement would be an option that a hospital and its medical staff could elect to use at their discretion, we proposed language that states "the provisions of paragraphs (c)(5)(iii), (iv), and (v) do not apply to a medical staff that chooses to maintain a policy that adheres to the requirements of paragraphs (c)(5)(i) and (ii) for all patients." In other words, a hospital and its medical staff would be free to exercise their clinical judgment in determining whether a policy for identifying specific patients as not requiring a comprehensive $\mathrm{H} \& \mathrm{P}$ (or any update to it) prior to specific outpatient surgical or procedural services, and instead requiring only a pre-surgical assessment for these patients, would be their best course. Or, if a hospital and its medical staff decided against such a policy, then only the current H\&P and update requirements (at $\S \S 482.22$, 482.24 , and 482.51 ) would continue to apply and the proposed requirements for this CoP, as well as those proposed for $\S \S 482.24$ and 482.51, would not apply.

For the current CoP at $\S 482.24$,

"Medical Record Services," we specified that we would revise the provisions at $\$ 482.24(\mathrm{c})(4)(\mathrm{i})(\mathrm{A})$ and (B) regarding an $\mathrm{H} \& \mathrm{P}$ and its update to allow for an exception under proposed paragraph (c)(4)(i)(C) where we proposed to add a new requirement that, if applicable, the medical record would have to document assessment of the patient (in lieu of the requirements of paragraphs (c)(4)(i)(A) and (B)) after registration, but prior to surgery or a procedure requiring anesthesia services, for specific outpatient surgical or procedural services.

We also proposed to revise the current CoP, § 482.51, “Surgical Services," to allow for an exception to the requirements at $\S 482.51(\mathrm{~b})(1)$ (i) and (ii). Under proposed paragraph (b)(1)(iii), we proposed a new requirement that, prior to surgery or a procedure requiring anesthesia services and except in the case of emergencies, an assessment of the patient must be completed and documented after registration (and in lieu of the requirements of paragraphs (b)(1)(i) and (ii)). This proposed requirement would only apply in those instances when the patient is receiving specific outpatient surgical or procedural services and when the medical staff has chosen to develop and maintain a policy that identifies, in accordance with the requirements at $\S 482.22(\mathrm{c})(5)(\mathrm{v})$, specific patients as not requiring a comprehensive medical history and physical examination, or any update to it, prior to specific outpatient surgical or procedural services.

Comment: As reflected in the public comments for similar proposed changes for ASCs that we have previously discussed, the majority of comments submitted were supportive of the proposed changes that would give a hospital and its medical staff the flexibility to establish a policy for a presurgical or pre-procedural assessment of the patient (in lieu of the requirements of paragraphs (c)(5)(i) and (ii) for a comprehensive pre-surgical or preprocedural H\&P and its update), provided that the patient assessment is completed and documented after registration, but prior to surgery or a procedure requiring anesthesia services, and the patient is receiving specific outpatient surgical or procedural services as outlined in the policy. Several commenters stated that they appreciated the regulatory flexibility to establish specific patient policies such as these as long as they are based on recognized guidelines and best practices as well as on the clinical judgment of the medical staff. They stated that they believe such parameters are necessary to ensure patient health and safety while still allowing for reasonable methods to reduce the burden on both patients and providers, including the additional expense of pre-operative testing that is often performed unnecessarily on many patients undergoing only minor outpatient procedures and may be an unintended consequence of the requirement for a comprehensive $\mathrm{H} \& \mathrm{P}$ within 30 days of admission or registration for all hospital patients regardless of the surgery or procedure that they are undergoing.

Response: We thank the commenters for their support and agree that the flexibility provided by these revisions will reduce unnecessary regulatory burden affecting both patients and providers. We believe that it also has the potential to greatly reduce unnecessary costs associated with the current requirements for a comprehensive $\mathrm{H} \& \mathrm{P}$ for a specific class of patients undergoing low-risk outpatient surgeries and procedures for which there exist clear guidelines regarding the extent of pre-operative patient assessment and testing needed.

Comment: Some commenters either did not support these changes or had certain reservations about them, even though they supported the overall intent of the changes. One commenter stated that the change will not serve those beneficiaries with advanced illness well, recommended that the rule be revised to require that the assessment must be consistent with the patient's situation, medical complexity, and the proposed procedure, and believes that the requirements must err on the side of more, rather than less,

comprehensiveness. Another

commenter stated that while they appreciated CMS' recognition that the timing of H\&Ps may, in some instances, be duplicative and cause unnecessary burden, they were aware of cases where the current H\&P requirements prevented an adverse event. They also stated that the proposed revisions will be just as, and possibly more, burdensome than the current requirements; that CMS should consider comments before proceeding; and that, while they agree that there seems to be no evidence supporting a strict 30-day requirement, additional flexibility would be appreciated. One commenter stated that they believe the burden of assessing patients prior to surgery would be shifted from one provider (the primary care physician or the surgeon) to another (the anesthesiologist), and 
expressed concerns over the increased responsibility and liability that might be then imposed on an anesthesiologist (beyond his or her primary

responsibility for anesthesia services and care provided to a patient) for a surgery or procedure in which he or she was not the operating practitioner. A few commenters also expressed concerns over whether reimbursement requirements and rates would now change for outpatient surgeries and procedures that would only require an assessment and not a comprehensive $\mathrm{H} \& \mathrm{P}$, including concerns over which practitioner would now be reimbursed for the assessment (for example, the patient's primary care practitioner versus the operating practitioner).

Response: We appreciate the concerns raised by commenters and have thoroughly considered them. However, we must again note and emphasize to readers that this revision will be a regulatory option available to hospitals and one that a hospital and its medical staff must make the policy decision to exercise. We expect that this decision will be based on the clinical judgment and recommendations of the medical staff, which must be supported by nationally recognized evidence and guidelines for best practices in this area, in order for the hospital to determine if the best course would be to establish a policy for identifying specific patients as not requiring a comprehensive $\mathrm{H} \& \mathrm{P}$ (or any update to it) prior to specific outpatient surgical or procedural services, and instead require a more limited pre-surgical assessment for these patients. We expect that most hospitals and their medical staffs will perform risk/benefit analyses to inform their decisions. We also expect that a number of these hospitals, based on their analyses, will decide to maintain a policy that continues to follow the current $\mathrm{H} \& \mathrm{P}$ and update requirements (at $\S 482.22,482.24$, and 482.51) and will not choose to exercise this option in any way. Conversely, we also expect that some will choose to exercise this option fully and to the broadest extent possible while still remaining in compliance with the requirements finalized. We further expect that another significant subset of hospitals will fall somewhere in the middle in their policy decisions and will most likely elect to exercise this option within an even more narrow and stringent set of parameters than CMS is requiring here. The regulatory flexibility and framework of these final requirements will allow each hospital to establish and tailor its own policy parameters according to its specific patient populations, individual institutional needs and resources, and own medical staff recommendations as long as the policies and procedures established and implemented meet or exceed the requirements finalized in this rule. As finalized here, these requirements, while providing a hospital with an alternative and less burdensome approach to pre-surgical patient assessment, will also at the same time ensure that a hospital takes into consideration all patient safety factors and quality of care issues, such as the degree of complexity of the patient's medical condition as well as that of the planned procedure itself, when it establishes a process to identify those patients to whom such a policy would apply.

In response to the commenter who stated that, under this new option, the assessment of patients prior to surgery will be "shifted from one provider (the primary care physician or the surgeon) to another (the anesthesiologist)," we note that the Anesthesia services CoP contains a separate provision (separate and distinct from the H\&P, update, and pre-surgical assessment requirements in the Surgical services CoP) that requires that a ". . . preanesthesia evaluation [be] completed and documented by an individual qualified to administer anesthesia . . ." and that it must be “. . . performed within 48 hours prior to surgery or a procedure requiring anesthesia services." The anesthesiologist is responsible for this evaluation, but not for the H\&P, update, and pre-surgical assessment requirements that we are finalizing here While an anesthiologist could certainly qualify to perform any of these presurgical assessments, we expect the operating practitioner, who is also responsible for the pre-, intra-, and postoperative care of the patient and must be a physician (as defined in section 1861(r) of the Act), an oral and maxillofacial surgeon, or, in accordance with State law and hospital policy, another qualified licensed individual (who would most likely be a member of the operating practitioner's team, such as an NP or PA, and who, by extension, would also be responsible for the care of the patient) to perform the presurgical assessment as required in this final rule. If a hospital and its medical staff choose to establish policies and procedures that delineate the duties and responsibilities of any individual anesthiologist (or any individual qualified to administer anesthesia) to include performance of the pre-surgical assessments included under this rule, then the hospital would need to demonstrate that these pre-surgical assessments are separate and distinct from the pre-anesthesia evaluations of patients required at $\S 482.52$.

Furthermore, a hospital must also ensure that any such policies and procedures, which assign these presurgical assessment duties and responsibilities to an individual anesthiologist (or an individual qualified to administer anesthesia) as discussed here, are not only in accordance with State law, but are also consistent in this regard with the current standards of both anesthesia care and surgical care.

The comments regarding reimbursement requirements and rates for outpatient surgeries and procedures are outside the scope of the CoPs and this rule.

Comment: A few commenters were concerned about compliance with the revised requirements if no clear and recognized guidelines or

recommendations exist for pre-surgical patient assessment for specific classes of patients undergoing certain outpatient surgeries and procedures.

Response: The revised requirements, which allow for the option of establishing a policy for identification of specific patients to whom the assessment requirements in $\S 482.22$ (c)(5)(iii) would apply, are conditioned upon a hospital and its medical staff demonstrating evidence that the specific parameters required in this final rule are met. A hospital and its medical staff should not include those classes of patients and those outpatient surgeries and procedures in its pre-surgical patient assessment policy if the hospital finds that it cannot meet the requirements we are finalizing at $\S 482.22(\mathrm{c})(5)(\mathrm{v})$, including the requirement that the medical staff must demonstrate evidence that its policy is based on nationally recognized guidelines and standards of practice for the assessment of specific types of patients prior to specific outpatient surgeries and procedures.

Final Rule Action: We are finalizing the requirements in $\S \S 482.22,482.24$, and 482.51, with only minor

modifications. Specifically, we are changing the term "oromaxillofacial surgeon" to the correct term of "oral and maxillofacial surgeon" where indicated.

Contact: CAPT Scott Cooper, USPHS, 410-786-9465.

c. Medical Staff: Autopsies (§ 482.22(d))

We proposed to remove the requirement at $\S 482.22(\mathrm{~d})$, which states that a hospital's medical staff should attempt to secure autopsies in all cases of unusual deaths and of medical-legal 
and educational interest. The mechanism for documenting permission to perform an autopsy must be defined and there must be a system for notifying the medical staff, and specifically the attending practitioner, when an autopsy is being performed.

Comment: Several commenters agreed with the proposal, which they stated would remove duplicative administrative work and allow hospitals to defer to State requirements when an autopsy is necessary. Many commenters, including national associations representing medical examiners and pathologists, disagreed with the removal of the requirement that a hospital's medical staff attempt to secure autopsies for unusual deaths or educational purposes. The commenters stated that hospitals should attempt to obtain family permission for autopsies related to deaths resulting from questions about efficacy of treatment, for educational purposes, or for issues of unintended outcomes of treatment or medical uncertainty and these commenters also expressed concern that the removal of this proposal would lead to a further reduction in an already low national autopsy rate.

However, many of these commenters stated that hospitals should not be required to attempt to obtain family permission, or perform autopsies, in cases of medical-legal interest. In those circumstances, the commenters stated, hospitals should report the death to, and consult with, the authority of their local medical examiner, coroner, or medicolegal death investigative authority.

Finally, one commenter requested that CMS specifically state that hospitals are not prohibited from performing autopsies.

Response: We agree that hospitals should not attempt to secure autopsies in medical-legal cases without first contacting their State's medical examiner or medical authority, in accordance with their State's laws. We will defer to state law on this issue, since each State has their own standards and laws regarding the performance of autopsies for medical-legal purposes, and we therefore are removing this as a requirement in the CoPs for hospitals. Furthermore, we believe that it is appropriate to remove the duplicative and burdensome requirement that hospitals attempt to secure autopsies for other cases of unusual deaths or for educational interest. We clarify that removing this requirement would not prohibit hospitals from performing autopsies and we believe that hospitals will implement their own policies regarding autopsies. While we understand the commenter's concerns regarding the decline in the national autopsy rate, we disagree that the removal of this specific requirement will cause a measurable decrease in the autopsy rate, impact quality of care, or dissuade hospitals from performing autopsies. As commenters themselves have noted, there are various causes that may have contributed to the reduction in the autopsy rate including risk adversion due to litigation concerns and concerns about reimbursement rates, and we have no additional evidence that would lead us to the conclusion that the removal of this requirement would exacerbate these numbers. We therefore are finalizing our proposal to remove the requirements at $\S 482.22(\mathrm{~d})$.

Although we are finalizing our proposal, we note that the removal of this requirement should not be construed as a diminution of our support for hospitals continuing to perform autopsies for various purposes, and we encourage hospitals to establish policies regarding autopsies, where appropriate.

Comment: A few commenters suggested that all hospital admissions require the patient (or his or her representative) to affirmatively allow or prohibit an autopsy in the event of death. One commenter also stated that autopsies should be required for any hospital death, unless explicitly rejected by next of kin.

Response: Mandating that hospitals perform autopsies, or that hospitals ask permission to perform an autopsy upon a patient's admission, would be unduly burdensome to hospitals and contrary to the purpose of the CoPs, which establish baseline health and safety requirements. However, hospitals may choose to establish their own policy that would require patients or their representatives to permit or decline autopsies upon admission, if they believe such a requirement is appropriate. As we previously stated, there is no prohibition against autopsies and hospitals are free to enact policies regarding autopsies if they choose to do so.

Additionally, requiring hospitals to perform autopsies could potentially conflict with State and local laws regarding autopsies for medical-legal cases. For instance, certain State laws require that hospitals report deaths arising from medical-legal circumstances to their local medical examiner or other authoritative body, so that a determination can be made as to whether an autopsy must be performed.

Final Rule Action: We are finalizing the proposal to remove $\S 482.22(\mathrm{~d})$, without modification.
Contact: Alpha-Banu Wilson, 410786-8687.

\section{d. Infection Control (§ 482.42)}

We proposed a new standard at § 482.42(c), "Unified and integrated infection control program for multihospital systems." Like the proposed requirements for a unified and integrated QAPI program, the proposed standard for infection control would allow that for a hospital that is part of a hospital system consisting of multiple separately certified hospitals subject to a system governing body legally responsible for the conduct of each hospital, such system governing body could elect to have a unified and integrated infection control program for all of its member hospitals after

determining that such a decision was in accordance with all applicable State and local laws. The system governing body would be responsible and accountable for ensuring that each of its separately certified hospitals met all of the requirements of this section. Each separately certified hospital subject to the system governing body would have to demonstrate that the unified and integrated infection control program: (1) Was established in a manner that took into account each member hospital's unique circumstances and any significant differences in patient populations and services offered in each hospital; (2) established and implemented policies and procedures to ensure that the needs and concerns of each of its separately certified hospitals, regardless of practice or location, are given due consideration; (3) had mechanisms in place to ensure that issues localized to particular hospitals are duly considered and addressed; and (4) designated a qualified individual(s) at the hospital with expertise in infection prevention and control to be responsible for communicating with the unified infection control program, for implementing and maintaining the policies and procedures governing infection control, and for providing infection prevention education and training to hospital staff.

Comment: Most commenters supported the proposal to allow hospitals that are part of a multihospital system to have a unified and integrated infection control program. The proposed rule included a specific request for public comment on whether there are any other programs currently required under the CoPs for each separately certified hospital, beyond the QAPI and Infection control programs proposed here, that stakeholders believe would likewise be better managed under a system governing body legally 
responsible for the conduct of each separately certified hospital. In response, we received comments asking CMS for further revisions to the CoPs, like those proposed for QAPI and infection control programs here (and with specific mention of revising the Nursing services CoP in this way), to allow for similar departmental and operational integration among hospitals within a multi-hosptial system with a single governing body. The commenters stated that expansion of this flexibility for other hospital services, departments, units, and programs would reduce operational burden for individual hospitals, ensure the proper level of staff expertise for member hospitals, and improve the quality and continuity of care for all patients served within the system. A few commenters also expressed appreciation for the expanded flexibility that this proposal would afford hospitals by reducing burden, increasing efficiencies, and eliminating the duplication of efforts.

One commenter encouraged CMS to apply this approach to situations when a multi-hospital system's providers have to fulfill additional requirements stemming from Medicaid or Medicare managed care plans or other external regulatory entities. The commenter suggested the mandated training related to the special needs plan models of care (42 CFR 422.101(f)(2)(ii)) as an example of how this could be applied. The commenter stated that a multihospital system with a unified infection control program as allowed under the requirements finalized in this rule, and that is also potentially participating in an ACO, would most certainly meet the Model of Care training requirement. This commenter also suggested an alternative approach where ACO participants would be deemed as meeting the Model of Care requirement for all other external regulatory entities by meeting the unified infection control program requirements finalized here.

Response: We thank the commenters for their support. We believe that a hospital's governing body should be afforded the option of unifying and integrating the various member hospitals within their multi-hospital system into a unified infection control program. As we discussed for unified and integrated QAPI programs, such a model would incorporate each individual hospital's infection control program, which would enable increased efficiencies, innovations, provider flexibility, and allow for the dissemination of best practices for patient care while also potentially improving patient safety and outcomes. We also believe that a unified infection control model is a natural progression for a multi-hospital system that utilizes a system governing body (as allowed at $\S 482.12$ ), a unified medical staff (as allowed at $\S 482.22$ ), and a unified QAPI program (as finalized in this rule at $\S 482.12)$

The comments and recommendations regarding the application of the unified infection control model and its CoP requirements to any additional requirements mandated by Medicare and Medicaid managed care plans or other external regulatory entities are outside the scope of the CoPs and this rule.

Final Rule Action: We are finalizing the proposed requirements in $\S 482.42$. Moreover, in addition to revisions proposed and finalized for the Hospital/ CAH Innovation Rule regarding Antibiotic Stewardship Programs (ASPs) (now part of the Infection Prevention and Control CoP discussed in Section III.B.6. of this final rule and finalized here at $\S 482.42$ ), we are finalizing changes to $\S 482.42$ that will now address the designated and qualified individual(s) at the hospital responsible for communicating with the unified infection control program, for implementing and maintaining the policies and procedures governing infection control, and for providing infection prevention education and training to hospital staff with regard to the ASP as well. We are also making other minor modifications to this section to finalize changes proposed in the Hospital/CAH Innovation Proposed Rule. All of these changes are discussed later in Section III.B.6. of this final rule.

Contact: CAPT Scott Cooper, USPHS, 410-786-9465.

e. Special Requirements for Hospital Providers of Long-Term Care Services ("Swing-Beds") (§ 482.58(b)(1), (4), (5), and (8), and Identical CAH

Requirements: § 485.645(d)(1), (4), (5), (6), and (7))

Hospitals providing swing-bed services must meet all of the requirements at 42 CFR part 482 , which includes the swing-bed requirements at $\S 482.58$ for patients receiving swingbed services, and CAHs providing swing-bed services must meet all of the requirements at 42 CFR part 485 , subpart $\mathrm{F}$, which includes the swingbed requirements at $\S 485.645$ for patients receiving swing-bed services. The swing-bed requirements within the hospital and CAH CoPs include a subset of cross-referenced long-term care requirements contained in 42 CFR part 483, subpart B, for which hospital and CAH swing-bed providers are surveyed as they are for all of the CoPs in their respective programs. We have determined that some of the crossreferenced long-term care requirements for hospitals and CAH swing-bed providers are unnecessary and unduly burdensome, given their focus on "residents" and longer length of stays, which we believe are not relevant to swing-bed patients. Thus, we proposed to remove the following requirements: $\S \S 482.58(\mathrm{~b})(1)$ and (c) and 485.645(d)(1) (incorporating long-term care facility requirements at $\S 483.10$ (f)(9)). Under our current regulations at $\S 483.10$ (f)(9), the resident has a right to choose to, or refuse to, perform services for the facility, and the facility must not require a resident to perform services for the facility. Regulations at $\S \S 482.58(\mathrm{~b})(1)$ and $485.645(d)(1)$ incorporate this resident right by reference.

We expect hospital and CAH swingbed providers who do offer patients the option of providing services for the facility to have current policies and procedures that reflect this policy that includes protocol for establishing an agreement between the two parties.

Comment: Commenters universally supported the proposal to remove the provision requiring hospitals and $\mathrm{CAH}$ swing-bed providers to provide residents with the right to choose to, or refuse to, perform services for the facility, and not requiring a resident to perform services for the facility. As with the majority of the hospital and CAH swing-bed proposals, commenters noted that this requirement is unnecessary, the source of confusion, or is unduly burdensome.

Response: We appreciate the comments received and continue to believe that this change is appropriate.

Final Rule Action: We are finalizing this proposed change without revisions.

$\S \S 482.58(b)(4)$ and $485.645(d)(4)$ (incorporating long-term care facility requirements at \$483.24(c)): The facility must provide, based on the comprehensive assessment and care plan and the preferences of each resident, an ongoing program to support residents in their choice of activities and the activities program must be directed by a qualified professional who is a qualified therapeutic recreation specialist or an activities professional. Patients receiving swing-bed services in a hospital or CAH are not long term residents of the facility and generally only receive swing-bed services for a brief period of time for transition after the provision of acute care services. We expect that for those patients who receive swing-bed services for an extended period of time, their nursing care plan-as required under $\S 482.23(\mathrm{~b})(4)$ for hospitals and 
$\S 485.635(d)$ (4) for CAHs-is based on assessing the patient's nursing care needs and will support care that holistically meets the needs of the patient, taking into consideration physiological and psychosocial factors.

Comment: The majority of commenters were supportive of the proposed removal of the requirement for hospital and CAH swing-bed providers to provide an ongoing program to support residents in their choice of activities and have an activities program that is directed by a qualified professional. As with the majority of the hospital and CAH swing-bed proposals, commenters noted that this requirement is unnecessary, the source of confusion, or is unduly burdensome due to the limited length of stay for most patients receiving swing-bed services. Commenters generally agreed that the activity needs of those patients who receive swing-bed services for an extended period of time would be met via the hospital and $\mathrm{CAH}$ nursing care plan requirements.

However, one commenter noted that in the event a swing-bed patient receives care for an extended period of time, the nursing care plan will not include interest-based group and individual activities that support the patient's physical, mental and psychosocial well-being. The commenter noted that therapeutic or recreational activities differ significantly from the goals that normally would be identified in a nursing care plan.

Response: We appreciate the comments received and continue to believe that this change is appropriate. It is expected that hospitals and CAHs, using an interdisciplinary approach, are providing services that meet the needs of all of their patients, including those receiving swing-bed services, regardless of their length of stay. In addition, nursing care plans are intended to provide direction on the type of nursing care the needed by the patient, stemming from the patient's diagnoses, that is organized based on the specific needs of the patient. The care plan is dynamic and should change as the needs of the patient change. As a result, if the needs of the patient include interest-based group and individual activities that support the patient's physical, mental and psychosocial wellbeing, we expect that the hospital or $\mathrm{CAH}$ will provide these services to the patient.

Final Rule Action: We are finalizing this proposed change without revision. $\S \S 482.58(b)(5)$ and $485.645(d)(5)$ (incorporating long-term care facility requirements at \$ 483.70(p)): Any facility with more than 120 beds must employ a qualified social worker on a full-time basis.

In accordance with the hospital and CAH swing-bed requirements, hospital swing-bed providers are not permitted to have more than 100 beds while CAH swing-bed providers are not permitted to have more than 25 beds for the provision of inpatient or swing-bed services. Based on feedback from stakeholders, removing this requirement would eliminate confusion for providers and accreditation organizations.

Comment: Commenters universally supported the proposal to remove the provision requiring hospitals and $\mathrm{CAH}$ swing-bed providers with more than 120 beds to employ a full-time social worker. As with the majority of the hospital and CAH swing-bed proposals, commenters noted that this requirement is unnecessary, the source of confusion, or is unduly burdensome.

Response: We appreciate the comments received and continue to believe that this change is appropriate.

Final Rule Action: We are finalizing this proposed change as proposed.

$\S \S 482.58(b)(7)$ and $485.645(d)(7)$ (incorporating the long-term care facility requirement at \$ 483.55(a)(1)): Under our long-term care facility requirements, the facility, must provide routine and emergency dental services to meet the needs of each resident, or obtain them from an outside resource, in accordance with $\S 483.70(\mathrm{~g})$.

Hospitals and CAHs are required to provide care in accordance with the needs of the patient that have been identified in such patients' plans of care; this could include non-emergency dental care. We expect that hospital swing-bed providers are currently addressing the emergent dental care needs of their patients under the existing hospital CoP at $\S 482.12(f)(2)$, which requires that hospitals have written policies and procedures for appraisal of emergencies, initial treatment, and referral when appropriate. Similarly, we expect that CAH swing-bed providers are currently addressing the emergent dental care needs of their patients under the existing emergency services $\mathrm{CoP}$ at $\S 485.618$, which requires CAHs to provide emergency care necessary to meet the needs of its inpatients and outpatients. As a result, we believe that this portion of the requirement is duplicative, given the current CoP requirements.

Comment: Commenters universally supported the proposal to remove the provision requiring hospitals and $\mathrm{CAH}$ swing-bed providers provide or obtain from an outside resource routine and emergency dental services to meet the needs of each resident. As with the majority of the hospital and CAH swingbed proposals, commenters noted that this requirement is unnecessary, the source of confusion, or is unduly burdensome.

In addition, the most recent guidelines from the American Dental Association note that patients should have regular dental visits, with the frequency determined by their dentist to accommodate for the patients' current oral health status and health history (American Dental Association, https:// www.ada.org/en/press-room/newsreleases/2013-archive/june/americandental-association-statement-onregular-dental-visits).

Response: We appreciate the comments received and continue to believe that this change is appropriate.

Final Rule Action: We are finalizing this proposed change as proposed.

Contact: Kianna Banks, 410-7863498.

f. Special Requirements for Psychiatric Hospitals (§ 482.61(d))

We believe that as currently written and implemented, this requirement requires clarification regarding the language that progress notes "must be recorded by the doctor of medicine or osteopathy responsible for the care of the patient as specified in $\S 482.12$ (c), nurse, social worker and, when appropriate, others significantly involved in active treatment modalities." We believe that nonphysician practitioners, including physician assistants, nurse practitioners, psychologists, and clinical nurse specialists, when acting in accordance with State law, their scope of practice, and hospital policy, should have the authority to record progress notes of psychiatric patients for whom they are responsible. Therefore, we proposed to allow the use of non-physician practitioners or MD/DOs to document progress notes of patients receiving services in psychiatric hospitals.

Comment: Commenters were mostly supportive of the proposal to clarify the documentation requirements for recording progress notes in the patient's medical records for patients receiving services in psychiatric hospitals. Commenters noted that the proposed change would reduce barriers for care providers and will give non-physician practitioners expanded access to document the provision of the health care to patients, resulting in improved continuity of care.

Response: We appreciate the comments received and continue to believe that this change is appropriate. 
Comment: One commenter opposed the proposed change, noting that the existing regulatory language already permits non-physician practitioners to document progress notes in the patient's medical records for patients receiving services in psychiatric hospitals; therefore, the change would be unlikely to produce costs savings from incorporating psychologists or other licensed practitioners in this requirement. Another commenter opposed the inclusion in the proposed rule of psychologists in the list of nonphysician practitioners allowed to document the patient's progress notes. The commenter notes that the current regulations permit psychologists to document the services they provide (psychotherapy, psychological/ neuropsychological testing notes), but they should not be granted the authority to write medical progress notes due to the current Medicare regulations under $\S 482.12$ (c)(1)(vi).

Response: While we agree with the commenter regarding the intent of the current regulatory language, we believe that there is a need to clarify the intent of the language and ensure that the healthcare providers with the authority to document the progress reports are clearly identified. The majority of commenters supported the need for clarification due to the failure of the regulatory text to specify the nonphysician practitioners who could document progress reports.

Additionally, we believe psychologists are appropriately included in the list of non-physician practitioners who have the authority to document in progress notes for patients receiving services in psychiatric hospitals. The regulation at $\S 482.12$ (c)(1)(vi), referenced by the commenter, states that a clinical psychologist, as defined in $\S 410.71$, has the authority to admit patients and oversee the care of Medicare patients (but only with respect to clinical psychologist services as defined in $\S 410.71$ of this chapter and only to the extent permitted by State law).

Furthermore, non-physician practitioners currently document in the progress notes of patients in acute care hospitals to the extent of their scope of practice and hospital policy.

We believe that the revised language in this final rule will clarify our original intent in the proposed rule (as understood by readers as evidenced by the comments discussed here that request and support such clarification on this issue) that non-physician practitioners, including physician assistants, nurse practitioners, psychologists, and clinical nurse specialists, when acting in accordance with applicable State scope of practice laws, and hospital policies, should have the authority to record progress notes for all psychiatric and medical problems, which are present upon patient admission or which develop during hospitalization, for any psychiatric patient for whom these licensed practitioners are responsible as long as such patient problems fall within the bounds of a licensed practitioner's specific State scope of practice laws and hospital policies.

Comment: One commenter requested that clarification be provided regarding the use of the phrase "hospital policy" as it relates to the requirement that nonphysician practitioners act in accordance with hospital policy.

Response: Psychiatric hospitals must comply with the hospital CoPs under 42 CFR part 482, which includes the requirements for psychiatric hospitals. The hospital CoPs require that the hospital's governing body approve all hospital policies, and in accordance with $\S 482.12(a)(4)$, the governing body must determine (in accordance with State law) which categories of practitioners are eligible candidates for appointment to the medical staff. The governing body is required to appoint members of the medical staff after considering the recommendations of the existing members of the medical staff and approve medical staff bylaws and other medical staff rules and regulations. Non-physician practitioners, whether employees or contractors, would be subject to all rules, regulations, and policy manuals utilized by the hospital.

Final Rule Action: We are finalizing the changes as proposed.

- Contact: Kianna Banks, 410-7863498

\section{Transplant Centers}

a. Special Requirement for Transplant Centers ( $\S 482.68$ and 482.70)

We proposed to update the terminology within the hospital regulation at part 482 and the transplant regulations at $\S \S 482.68,482.70,482.72$ through 482.104 , and at $§ 488.61$, for clarification and consistency. Specifically, we proposed a nomenclature change which would:

- Replace the term transplant "center" in the regulation language with transplant "program" (each organ type would be a transplant program). A transplant program is located within a transplant hospital that provides transplantation services for a particular type of organ. Since individual transplant programs are surveyed for compliance with the CoPs, using the term transplant program throughout the regulation better aligns with current surveyor practice and will reduce provider confusion. In order to provide further clarity, we also proposed to update the definitions at $\S 482.70$.

- Consistently use Independent Living Donor Advocate (ILDA) throughout the regulation.

- Change "beneficiaries" to "recipients".

Comment: All comments we received expressed support for the proposed nomenclature change, which would make the terminology used in the regulations consistent with the terminology used by the Organ Procurement and Transplantation Network (OPTN) and the transplant community.

Response: We thank the commenters for their support. We are finalizing this proposal without modification.

b. Data Submission, Clinical Experience, and Outcome Requirements for ReApproval of Transplant Centers (§ 482.82)

We proposed to remove the requirements at $\S 482.82$ that require transplant centers to submit data (including, but not limited to, submission of the appropriate OPTN forms for transplant candidate registration, transplant beneficiary registration and follow-up, and living donor registration and follow-up), clinical experience, and outcome requirements for Medicare re-approval, and make conforming changes to $\S 482.102(\mathrm{a})(5)$ " Condition of participation, Patient and living donor rights" and § 488.61 "Special Procedures for Approval and ReApproval of Organ Transplant Centers.'

Comment: Most commenters, including several major organizations which represent the interests of transplant surgeons and other professionals, transplant patients, individual transplant programs, members of the transplant community, and the OPTN, strongly supported the proposal to remove the provision that requires transplant centers that are applying for Medicare re-approval to meet all data submission, clinical experience, and outcome requirements in order to be re-approved. These commenters agreed with our analysis of the unintended consequences that have occurred because of the Medicare reapproval requirements and many agreed that eliminating this requirement would improve transplantation in the United States. Many of these commenters also stated their belief that the proposal would reduce administrative burdens. A 
few commenters also supported maintaining the requirements for initial Medicare approval for transplant centers.

Several commenters, including members of the transplant community and interested individuals, objected to the removal of this proposal. These commenters as a whole were concerned that removing this proposal would affect quality of care and patient outcomes. Specifically, commenters were concerned as to whether information on transplant centers outcomes would continue to be available to the public, and whether CMS would still be able to identify underperforming transplant programs, in order to ensure patient safety and continued positive outcomes. Other commenters stated that, absent these requirements, CMS would rely upon transplant programs notifying CMS of changes rather than having a process in place that would monitor such changes. A few commenters expressed concerns regarding how CMS would identify underperforming transplant programs and requested guidance as to how CMS plans to ensure patient safety and positive outcomes by using the QAPI program instead of the current re-approval process. Others requested clarification as to how CMS would continue to monitor outcomes absent this requirement.

Response: We appreciate the commenters' support. CMS is committed to ensuring that our regulations support a patient's access to lifesaving organs. We also strive to protect the quality of care that the transplant recipient and donor receives while in the transplant facility and we agree with commenters that the removal of this requirement will work towards achieving these goals. We appreciate the numerous studies and professional opinions that were submitted by the public that further bolstered our understanding of the unintended consequences that have occurred as a result of the Medicare re-approval requirements for transplant centers and we therefore are finalizing our proposal to remove these requirements at $\S 482.82$. We believe that the removal of these requirements will lead to improved patient outcomes, increased transplantation opportunities for patients on the waitlist, improved organ procurement for transplantation, greater organ utilization, and reduced burden on transplant programs. We note that the removal of these requirements directly aligns with our goal to increase access to kidney transplants by increasing the utilization of available organs from deceased donors and reducing the organ discard rate, which we outline in the Advancing American Kidney Health Paper, which can be found on the Office of the Assistant Secretary for Planning and Evaluation website at https://aspe.hhs.gov/pdfreport/advancing-american-kidneyhealth.

We understand the concerns that commenters raised regarding the availability of transplant program outcome data and we remind commenters that transplant outcomes will still be available to the public every six months on the Scientific Registry for Transplant Recipients (SRTR) website at https://www.srtr.org/. In addition, CMS will continue to survey the program's QAPI program to make sure the program is tracking adverse events, performing thorough analysis of each adverse event, and that performance improvement projects ensure adverse events do not recur. CMS will also do complaint investigations based on public or confidential reports about outcomes or adverse events.

It is our expectation that transplant programs will use their QAPI programs to continue to monitor quality of care, evaluate transplantation activities and outcomes, and conduct performance improvements when necessary. We believe that these efforts and the survey of the CoPs provides sufficient oversight to ensure that transplant programs will continue to achieve and maintain high standards of care.

Comment: A few commenters, who were generally supportive of the proposals, had additional clarifying questions for CMS about the survey process. One commenter asked whether additional reporting on the part of the hospital and transplant based QAPI programs would be required. A few commenters asked whether CMS would monitor hospital and QAPI based programs through a different mechanism, while one comment asked whether CMS will be providing published information regarding these reviews. One commenter also expressed their opposition to a change to the transplant QAPI regulations, and they expressed concern that changing these regulations will have unintended negative consequences on transplant survival outcomes, safety issues, and an increased focused on transplant volume by programs. Another commenter asked the following questions:

- Whether the monitoring schedule for CMS surveys of transplant programs will remain the same;

- What circumstances will trigger a review from CMS outside of routine recertification surveys; and

- What options will be available to a transplant program with condition level deficiencies on recertification surveys once the mitigating factors and SIAs are removed.

Response: We did not propose changes to the transplant program QAPI requirements and, consistent with other provider types, there is no public reporting for the hospital and transplant QAPI programs. Transplant programs must continue to abide by the hospital and transplant program QAPI CoPs at $\S \S 482.21$ and 482.96, respectively. On survey, documentation of communication between these QAPI entities is expected and the hospital QAPI program should report to the Governing Body any issues with transplant outcomes.

In response to the questions about the survey process, we note that the survey interval will not change, and that public or confidential reports may trigger a complaint survey. Mitigating factors and systems improvement agreements were for outcomes non-compliance only and are therefore unnecessary with the removal of the outcomes re-approval requirement at $\S 482.82$.

Comment: A few commenters were generally supportive of CMS's goals to improve organ transplantation by removing provider disincentives, but the commenters suggested that this could be achieved through improvements to the quality and outcomes measures. Specifically, the commenters suggested that reported outcomes focus on long term outcomes instead of short term outcomes, data on waitlist survival, donor utilization, total volume of organs transplanted, transplant rate utilization, costeffectiveness, and other quality of care measures.

Response: We believe that the wide variety of data and studies presented in the proposed rule regarding the unintended consequences of the reapproval requirements sufficiently demonstrates that it is no longer appropriate to include specific outcome measures as a requirement for Medicare re-approval. Transplant programs, however, will still need to abide by these outcome measures for initial Medicare approval.

Comment: One commenter opposed the transplant center proposals and suggested that CMS look at Organ Procurement Organization (OPO) performance in producing quality organs.

Response: We thank the commenters for their feedback regarding OPO performance measures. However, we note that comments regarding OPOs are outside the scope of this final rule. 
c. Special Procedures for Approval and Re-Approval of Organ Transplant Centers (§ 488.61(f) Through (h))

We proposed to remove the requirements at $\S 488.61(\mathrm{f})$ through $(\mathrm{h})$ for mitigating factors and transplant systems improvement agreements for the re-approval process for transplant centers. This change is complementary to the proposed removal of $\S 482.82$, described previously.

Comment: The majority of commenters were supportive of the proposal to remove the mitigating factors and systems improvement agreements requirement for the reapproval process for transplant centers. These commenters stated that the removal of this requirement will relieve undue burden on transplant programs. However, a few commenters opposed the removal of this provision. The commenters were concerned that the removal of this provision would negatively impact programs and they noted that programs that failed to meet the re-approval requirements would be terminated, which would limit patient access. The commenter suggested that, if this proposal is finalized, CMS should monitor the number of programs that have been decertified or that will face decertification based on conditions.

Response: The proposed change to remove the mitigating factors and systems improvement agreements afforded to transplant centers for Medicare re-approval is a complementary change to the removal of the Medicare re-approval requirements in $\S 482.82$. We are finalizing the removal of $\S 482.82$, making the existence of the mitigating factors and systems improvement agreement requirements obsolete. However, we note that transplant programs are still afforded the opportunity to submit mitigating factors or enter into systems improvement agreements for the initial Medicare approval, and that we are not making additional changes to the current processes other than the provisions regarding mitigating factors and systems improvement agreements.

Final Rule Action:

1. We are finalizing the proposal to make nomenclature changes throughout the transplant center regulations at $\S \S 482.68,482.70,482.72$ through 482.104 , and at $\S 488.61$, without modification.

2. We are finalizing the proposal to remove $\S 482.82$, without modification.

3 . We are finalizing the proposal to remove the mitigating factor and systems improvement agreement requirements for Medicare re-approval at $\S 488.61(\mathrm{f})$ through $(\mathrm{h})$, without modification.

Contact: Alpha-Banu Wilson, 410786-8687.

\section{Home Health Agencies}

We received 27 timely public comments on our proposed changes to the HHA requirements. Commenters included industry associations, healthcare systems, individual HHAs, consumer advocacy organizations, and clinicians. Overall, the majority of commenters were supportive of the goal of the proposed changes. Those comments are discussed below.

\section{a. Patient Rights (§ 484.50(a)(3) and} (c)(7))

We proposed to delete the requirement at $\S 484.50(\mathrm{a})(3)$ that HHAs must provide verbal notification of all patient rights. We proposed to limit the verbal notification requirements to those requirements set out in section

1891(a)(1)(E) of the Act for which verbal notification is mandatory. We proposed to revise $\S 484.50(\mathrm{c})(7)$ to implement this more limited verbal notification requirement. Revised § 484.50(c)(7) would require HHAs to verbally discuss HHA payment and patient financial liability information with each HHA patient as described above.

Comment: The majority of comments submitted regarding this topic expressed support for the proposed change to require written notice of patient rights for all enumerated rights, and oral notice only for those rights specifically set forth in the Act as requiring such oral notice. However, a small number of comments did not support this change, stating that oral notice of all rights, rather than only those set forth in the Act, has value to patients and caregivers. One commenter stated that oral notice is particularly important for individuals with lower literacy levels due to disabilities.

Response: Consistent with the notice of patient rights requirements for other outpatient provider types, such as hospices, ambulatory surgery centers, and community mental health centers, for which written notice of patient rights is the only requirement, and in light of the support for this proposed change expressed by the majority of commenters, we are finalizing this change. We are sensitive to concerns related to those individuals with lower literacy levels due to disabilities that may impact understanding of the notice of patient rights. We remind all HHAs that, as part of their Medicare provider agreements, and in accordance with the other requirements of $\S 484.50$, they are responsible for complying with the provisions of the Americans with Disabilities Act and Section 504 of the Rehabilitation Act when communicating with all patients regarding all subjects, including the notice of patient rights. HHAs must provide equal access to individuals with disabilities, including the provision of auxiliary aids and alternate formats, including, but not limited to, the provision of qualified interpreters, large print documents, Braille, digital versions of documents, and audio recordings.

\section{b. Home Health Aide Services} (§ 484.80(h)(3))

We proposed to eliminate the requirement at $\S 484.80(\mathrm{~h})(3)$ that HHAs conduct a full competency evaluation of home health aides, and replace it with a requirement to retrain the aide regarding the identified deficient skill(s) and require the aide to complete a competency evaluation related only to those skills.

Comment: Comments overwhelmingly supported the proposed change to remove the requirement that a home health aide must complete a full competency evaluation whenever a skill deficiency is noted during the aide supervision process.

Response: We continue to believe that this change is appropriate, and are finalizing it as proposed.

\section{c. Clinical Records (§ 484.110(e))}

We proposed to remove the requirement at $\S 484.110(\mathrm{e})$ that the requested clinical record copy must be provided at the next home visit, while retaining the requirement that the information must be provided within 4 business days.

Comments: Comments universally supported the proposal to remove the requirement that HHAs must provide to patients a copy of information contained in the clinical record by the time of the next HHA visit. A few comments explicitly supported maintaining the requirement to provide the requested information to patients within 4 days. However, other comments stated that the proposed change did not provide enough burden relief, and suggested that the requirement to provide a copy of such information within 4 days should also be revised to allow HHAs up to 30 calendar days to provide such information. Commenters stated that 4 business days was insufficient time to access records, which may be archived offsite, make copies, and send those copies in the mail to arrive within 4 business days at the patient's home. One comment stated that the regulations should not include any requirements for HHAs to provide patients with 
information from their own clinical records. Other commenters suggested that a shorter timeframe for providing information could be limited to only the information from the current 60 day episode of care, rather than to all certification periods from the episode of care or the patient's entire record of care that may cross several different episodes of care. Additionally, some commenters stated that HHAs should be permitted to charge patients a fee for providing information from the patient's own clinical record. However, other commenters specifically supported the prohibition on charging patients a fee to receive information from their own records.

Response: We appreciate the commenters' support for our proposed revisions, and for their suggestions for further changes regarding the HHA clinical records provisions. Addressing the evolving need for the electronic exchange of health information amongst health care providers and also between patients and their health care providers is an Administration priority. As such, we will consider the issues raised by commenters in the broader context of interoperability and health information exchange, and will use these comments to inform future rulemaking. We are not finalizing the changes to $\S 484.110(\mathrm{e})$ at this time.

\section{d. Additional Comments}

\section{Summaries of the additional}

suggestions that we received that are not directly related to our proposals and our responses are set forth below.

Comment: Several commenters suggested that the requirement for HHAs to provide certain specified information, such as the upcoming HHA visit schedule and information about the treatments being furnished by HHA clinicians (\$ 484.60(e)) in writing to patients, should be completely removed or significantly revised to remove most of the specified information from the list. Commenters specifically cited the requirement to provide patients with a visit schedule, contact information for a hospice clinical manager, and information about the treatments being provided as being overly burdensome requirements.

Response: While we understand the concerns expressed by commenters, we continue to believe that providing patient-centered, patient-directed care necessitates the provision of this crucial information to all patients. Patients cannot be active participants in their own care and advocates for their own interests without having essential information about when care will be provided to them, what treatments are being (or are supposed to be) administered during their care, and information for how to contact a clinical member of the HHA care team to discuss their questions and concerns. While it may be challenging for HHAs to keep patients abreast of their own care, such efforts form the basis of patient-centered care and cannot be ignored.

Comment: A commenter suggested that the CoP for the comprehensive assessment should be revised to permit a registered nurse or a therapist to perform the comprehensive assessment in all cases where both services are ordered. A few commenters suggested that HHAs should not be required to provide any clinical services by their own employees, per the requirements of $\S 484.105(\mathrm{f})$, and should instead be allowed to provide all clinical services under arrangement.

Response: Changes of this magnitude would mark a significant departure from longstanding CMS policy. As such, we believe that it would be most appropriate to use the traditional notice and comment rulemaking process to allow all interested parties the opportunity to comment on the concepts. We will take these suggestions under consideration for future rulemaking efforts.

Comment: Several commenters stated that nurse practitioners, in addition to physicians, should be allowed to write orders for the home health plan of care and provide care plan oversight.

Response: Section 1861(m) of the Act requires the HHA plan of care to be under the direction of a physician. Section 1861(r) of the Act defines "physician" in a manner that does not include other licensed practitioners, such as nurse practitioners and physician assistants. Therefore, pursuant to statute, other licensed practitioners may not establish and maintain the home health plan of care, including reviewing, signing, and ordering services on the home health plan of care.

Comment: A few commenters submitted comments related to physician signatures and communication with physicians regarding orders and the plan of care. Some comments stated that a physician signature should not be required for therapy orders. The commenters stated that requiring a physician signature on such orders delays the initiation of therapy services. Another comment stated that HHAs should not be required to communicate with all physicians who write orders for the plan of care when there is a change in the plan of care.
Response: In order to maintain appropriate oversight of the HHA plan of care, all HHA services, including therapy services, must be ordered by a physician (§ 484.60(b)(1)). The CoPs allow for verbal orders in order to facilitate a timely initiation of care, requiring that verbal orders be authenticated and dated by the physician in accordance with applicable state laws and regulations, and consistent with the HHA's own internal policies. Typically, a physician writes orders for a therapist to evaluate and treat the patient. The requirement for the physician order and subsequent signature in accordance with State law and HHA policy would not delay therapy services after the therapist's evaluation and recommended treatment plan has been communicated to the physician for approval. It is not necessary to withhold therapy services while waiting for the physician confirmation of the therapy plan.

We agree with the commenter that communicating with all involved physician(s) is not necessary for every single change in the plan of care. Section 484.60(c)(3) requires such communication only when the change to the plan of care is due to a change in the patient's health status (for example, initiating a new medication) or a change in the plan for the patient's discharge from the HHA. The communication of other changes that do not fall into one of these categories (for example, adjusting the dose of a current medication) is left to the discretion of HHA clinical staff and the clinical manager(s) responsible for the patient's care.

Comment: Numerous commenters submitted suggestions for changes to HHA payment policies, such as the face to face requirement and the homebound requirement, which they believe should be addressed as part of CMS burden reduction efforts. A single commenter suggested a revision to the Home Health Consumer Assessment of Healthcare Providers and Systems (HHCAHPS). A small number of commenters submitted comments regarding information in the HHA interpretive guidelines.

Response: Comments not related to the HHA CoPs are outside of the scope of this rule. Individuals wishing to submit comments regarding CMS payment policies may submit those comments as part of the annual HHA payment policy proposed rule. We have shared these unrelated comments with the appropriate components within CMS.

Final Rule Action:

1. We are finalizing the proposal to delete the requirement at $\S 484.50$ (a)(3) 
that HHAs must provide verbal

notification of all patient rights.

2 . We are finalizing the proposal to revise $\S 484.50$ (c)(7), requiring HHAs to verbally discuss HHA payment and patient financial liability information with each HHA patient.

3. We are finalizing the proposal to eliminate the requirement at $\S 484.80(\mathrm{~h})(3)$ to conduct a full competency evaluation, and replace it with a requirement to retrain the aide regarding the identified deficient skill(s), and require the aide to complete a competency evaluation related only to those skills.

4. We are not finalizing the proposal to remove the requirement at $\S 484.110(\mathrm{e})$ that the requested clinical record copy must be provided at the next home visit.

Contact: Danielle Shearer, 410-7866617.

7. Comprehensive Outpatient Rehabilitation Facilities (CORFs) Utilization Review Plan (§ 485.66)

We proposed to amend the utilization review plan requirements at $\$ 485.66$ to reduce the frequency of utilization reviews from a quarterly basis to an annual requirement.

We received two timely public comments on our proposed changes to the CORF requirements. Both comments expressed strong support for the proposed changes; therefore we are finalizing those changes as proposed in this final rule.

1. Final Rule Action: We are finalizing the proposal to revise $\S 485.66$, requiring the facility to have a written utilization review plan that is implemented annually, without modification.

Contact: CAPT Jacqueline Leach, USPHS, 410-786-4282.

\section{Critical Access Hospitals \\ a. Organizational Structure (§ 485.627(b)(1))}

We proposed to remove the requirement for CAHs to disclose the names and addresses of their owners, those with a controlling interest in the $\mathrm{CAH}$ or in any subcontractor in which the CAH directly or indirectly has a 5 percent or more ownership interest, in accordance with 42 CFR part 420 , subpart $\mathrm{C}$. This requirement is duplicative, as it is also a requirement for the provider agreement for Medicare participation. This proposal was also included in the Medicare and Medicaid Programs; Hospital and Critical Access Hospital (CAH) Changes To Promote Innovation, Flexibility, and Improvement in Patient Care; Proposed Rule (81 FR 39447) for the same reason.
Comment: Commenters universally supported the proposal to remove the CAH disclosure requirement, noting that the requirement duplicates a provision found elsewhere in our regulations. Comments received regarding this provision in the Medicare and Medicaid Programs; Hospital and Critical Access Hospital (CAH) Changes To Promote Innovation, Flexibility, and Improvement in Patient Care; Proposed Rule (81 FR 39447, 39460, June 16, 2016) were consistent with those received for this proposed rule, with commenters also universally supporting the proposal.

Response: We appreciate the comments received and continue to believe that this change is appropriate.

Final Rule Action: We are finalizing the proposed changes without modification.

Contact: Kianna Banks, 410-7863498.

b. Provision of Services ( $\$ 485.635(a)(4)$ )

Current regulations at $\$ 485.635$ require a CAH's professional healthcare staff to review policies and procedures annually; the review group must include one or more doctors of medicine or osteopathy and one or more physician assistants, nurse practitioners, or clinical nurse specialists. Based on our experience with other providers, we proposed a flexible approach that would allow CAHs to maintain their health and safety policies in such a manner as to achieve the intended outcomes for all patients. Thus, we proposed to change the requirement at $\S 485.635$ (a)(4) from "annual" to "biennial".

We received 20 public comments on our proposed changes to this $\mathrm{CAH}$ requirement. Commenters included hospital industry associations, individual providers, and national accrediting organizations. Overall the commenters were supportive of the proposed changes.

Summaries of the comments and our responses are set forth below.

Comment: All of the commenters agreed with the effort to reduce burden. However, a few of commenters suggested moving to a 3-year timeframe for reviews and several other commenters suggested aligning with hospital requirements and removing the timeframe and allow CAHs to determine when reviews are done.

Response: CAHs are rural providers with separate Conditions of

Participation from hospitals and they do not have the range or number of personnel, among other requirements we require for hospitals.

We believe that the approach of requiring a biennial review reduces burden while maintaining the appropriate safeguards for healthy outcomes for CAH patients. Therefore, we are finalizing this requirement without modification.

Final Rule Action: We are finalizing the proposed changes to $\S 485.635$ (a)(4).

Contact: Mary Collins, 410-786-3189.

c. Special Requirements for CAH

Providers of Long-Term Care Services

("Swing-Beds") (§ 485.645(d)(1), (4), (5) and (8))

The special requirements for $\mathrm{CAH}$ swing-bed providers are nearly identical to the requirements for hospital providers of swing-bed services. As a result, please refer to the discussion on the special requirements for hospital providers of swing-bed services under section II.D. 3 for the details of the proposed changes for these requirements for both hospitals and CAHs. We proposed the following revisions to the CAH swing-bed requirements:

- Revision of $\S 485.645(d)(1)$ to remove the cross-referenced long-term care requirement in $\S 483.10$ (f)(9), which requires that CAH swing-bed providers to offer residents the right to choose to or refuse to perform services for the facility and prohibits a facility from requiring a resident to perform services for the facility;

- Removal of $\S 485.645(d)(4)$, which requires $\mathrm{CAH}$ swing-bed providers to provide an ongoing activity program that is directed by a qualified therapeutic recreation specialist or an activities professional who meets certain requirements (cross-referenced long-term care requirement § 483.24(c));

- Revision of $\S 485.645(d)(4)$ (as redesignated) to remove the crossreferenced long-term care requirement $\S 483.70(\mathrm{p})$, which requires that $\mathrm{CAH}$ swing-bed providers with more than 120 beds to employ a qualified social worker on a full-time basis; and

- Revision of $\S 485.645(d)(7)$ (as redesignated) to remove the crossreferenced long-term care requirement $\S 483.55(\mathrm{a})(1)$, which requires $\mathrm{CAH}$ swing-bed providers to assist in obtaining routine and 24-hour emergency dental care to its residents.

Contact: Kianna Banks, 410-7863498.

\section{Community Mental Health Centers (§ 485.914(d))}

We require CMHCs, at $\S 485.914(d)(1)$, to update clients' comprehensive assessments every 30 days. We proposed to revise $\S 485.914(\mathrm{~d})(1)$ to require that the CMHC update each client's comprehensive assessment via the CMHC interdisciplinary treatment 
team, in consultation with the client's primary health care provider (if any), when changes in the client's status, responses to treatment, or goal achievement have occurred, and in accordance with current standards of practice. Additionally, at

$\S 485.914(\mathrm{~d})(2)$, we proposed to retain the minimum 30-day assessment update time frame for those clients who receive PHP services. We believe this proposed change will allow for the provider and client to choose a visit schedule that is appropriate for the client's condition and not cause extra work or time for documentation that is unnecessary. Ultimately, this proposed change may allow for greater flexibility for the provider and client, saving time for both.

We received 4 timely public comments on our proposed changes to the requirements at $\S 485.914(\mathrm{~d})$. Commenters included physicians, associations and health networks. Overall, the majority of commenters were supportive of the goal of the proposed changes. Summaries of the major issues and our responses are set forth below.

All of the comments expressed strong support for the proposed changes to $\S 485.914(\mathrm{~d})$; therefore, we are incorporating those changes as proposed in this final rule.

Comment: We received several comments in support of the proposed change to the CMHC update to the comprehensive assessment requirement. Most commenters agreed that, for patients admitted for non-PHP services, it made sense to allow patients care needs, responses to treatment and care goals to drive decisions about when a patient needs to have an updated assessment. Commenters also agreed that it was appropriate to keep the requirement to update to the comprehensive assessment every 30 days for PHP patients. One commenter raised a concern regarding the proposed update to the comprehensive assessment requirement changes as it relates to patients needing to transfer to the hospital emergency department. The commenter stated that some emergency departments receive patients directly from CMHCs for emergency mental health treatment, and that it is important for the treating physician in the ED to know what medications the patient is taking. A commenter agreed with the proposed change to the comprehensive assessment update requirement, and asked for CMS to consider making similar burden reducing changes to all the requirements for the "Persons centered active treatment plan” under $§ 485.916$.
Response: We appreciate all of the positive feedback on the proposed changes to remove the 30-day updated assessment timeframe for non-PHP patients, and are finalizing this proposal without change. We understand the concerns raised related to how this assessment change would impact CMHC patients who must be transferred to a hospital emergency room. In the CMHC CoPs under $\S 485.914(\mathrm{e})(5)(\mathrm{v})(\mathrm{A})-(\mathrm{E})$, we state that when a client becomes an immediate threat to the physical safety of themselves, staff or other individuals, the CMHC must document a description of the client's behavior and the intervention(s) used (including medications), alternatives or other less restrictive interventions attempted, the client's condition or symptom(s) that warranted the use of the restraint or seclusion, and the client's response to the intervention(s) used. Typically, patient transfers from a CMHC to an emergency room include a transfer note summarizing the above information, including all current medications and any PRN medications that were given prior to the transfer to the emergency room. Furthermore, we agree with the suggestion that conforming changes should be made to $\S 485.916$, because the requirements of $\S 485.914$ and 485.916 constitute a cycle of care, with assessment and care planning feeding into one another. However, because we did not propose any changes to the client centered active treatment plan CoP (§ 485.916), we are legally not permitted to make any changes in a final rule without proposing the change to the public in a proposed rule. Therefore we will not be amending the regulatory language in $\S 485.916$ but will consider proposing a change to the requirements at a future date.

Final Rule Action: We are finalizing the proposal to revise $\S 485.914(\mathrm{~d})$ that the CMHC must update each client's comprehensive assessment via the CMHC interdisciplinary treatment team, in consultation with the client's primary health care provider (if any), when changes in the client's status, responses to treatment, or goal achievement have occurred and in accordance with current standards of practice. For clients that receive PHP services, the assessment must be updated no less frequently than every 30 days.

Contact: CAPT Mary Rossi-Coajou, USPHS, 410-786-6051.

\section{Portable X-Ray Services}

(§§ 486.104(a) and 486.106(a))

We proposed to revise the personnel qualification requirements at $\S 486.104(\mathrm{a})(1),(2),(3)$, or (4) by removing school accreditation requirements and simplifying the structure of the requirements. We proposed that all operators of portable X-ray equipment would meet one of the following:

(1) Successful completion of a program of formal training in X-ray technology at which the operator received appropriate training and demonstrated competence in the use of equipment and administration of portable x-ray procedures; or

(2) Successful completion of 24 full months of training and experience under the direct supervision of a physician who is certified in radiology or who possesses qualifications which are equivalent to those required for such certification.

We proposed to update $\S 486.106$ (a)(2) (specific to portable $\mathrm{x}$-ray services) to cross reference the requirements at $\S 410.32$ instead of setting forth specific order requirements. We proposed to retain the requirement that the portable $\mathrm{x}$-ray order must include a statement on why it is necessary to perform a portable $\mathrm{x}$-ray as opposed to performing the study in a facility where $\mathrm{x}$-rays are more typically performed.

We received 9 timely public comments on our proposed changes to the portable $\mathrm{x}$-ray requirements. Commenters included long-term care facility associations, portable x-ray associations, portable x-ray suppliers, and health care systems. Overall, the majority of commenters were supportive of the goal of the proposed changes.

Summaries of the major issues and our responses are set forth below.

Comment: All of the comments received regarding our proposal to revise the personnel requirements for individuals who perform portable x-ray services supported the proposed revision. A single commenter suggested that option 2 , related to 24 full months of training and experience under the direct supervision of a physician, and should not be included because these training programs are no longer offered. Response: We agree with the comments that it is appropriate to revise the personnel requirements for individuals who perform portable x-ray services in a manner that focuses on the skills of the individual rather than the accreditation of the institution that provided the training, and we are finalizing this change. We do not agree that it is appropriate to eliminate the qualification option related to 24 full months of training and experience under the direct supervision of a physician. The fact that such programs are no longer offered does not mean that those individuals who completed such programs are no longer qualified to 
perform portable $\mathrm{x}$-ray services, and thus excluded from performing their job duties. Excluding those individuals would not benefit patient health and safety or patient access to portable x-ray services; and may, in fact, reduce the number of qualified portable x-ray technicians and negatively impact access to care.

Comment: All of the comments received regarding our proposal to revise the requirements for portable $\mathrm{x}$ ray orders supported the proposed revision. One commenter specifically supported, while another specifically disagreed with, the proposal to retain the requirement that each order must specify the reason that portable x-ray services are necessary.

Response: We agree with the comments that it is necessary and appropriate to revise the requirements for portable x-ray orders to align with the separate payment requirements for diagnostic imaging orders that also apply to portable x-ray services at $\S 410.32$, and are finalizing this change. We believe that it is appropriate to require documentation regarding why this unique service is necessary in place of the more traditional facility-based $\mathrm{x}$ ray service, and are continuing this longstanding element as part of the revised requirements for portable x-ray orders.

Comment: We received several comments related to Medicare payment policies and Medicare payment manuals related to portable $\mathrm{x}$-ray services. We also received a comment related to the 2018 Crosswalk for Medicare Provider/ Supplier to Healthcare Provider Taxonomy, and the Medicare provider and supplier enrollment process.

Response: These comments are outside of the scope of this rule, and have been shared with the CMS components that are responsible for these subject matter areas.

Final Rule Action: We are finalizing the changes to $\S \S 486.104(\mathrm{a})$ and 486.106(a)(2).

Contact: Sonia Swancy, 410-7868445.

11. Rural Health Clinics (RHCs) and Federally Qualified Health Centers (FQHCs)

a. Provision of Services (§ 491.9(b)(4))

We proposed to change the requirement at $\S 491.9(\mathrm{~b})(4)$, related to reviewing patient care policies, from an "annual" review to a "biennial" review.

\section{b. Program Evaluation (§ 491.11(a))}

We proposed to revise the current requirement at $\S 491.11$ (a) by changing the frequency of the RHC or FQHC evaluation from annually to every other year.

We received 30 timely public comments on our proposed changes to the RHC and FQHC requirements. Commenters included industry associations, healthcare systems, individual RHCs and FQHCs and clinicians. Overall, the majority of commenters were supportive of the goal of the proposed changes. Summaries of the major issues and our responses are set forth below.

Comment: Overall, the majority of comments submitted regarding this topic expressed support for both of the proposed changes to require biennial provision of services policy reviews and clinic or center total program evaluation. Some of the commenters were completely supportive of the proposed biennial change, while some of the commenters stated they were unsure whether it will provide meaningful burden reduction. Other commenters were appreciative of the CMS goal to reduce burden on the RHC or FQHC and stated that the flexibility and opportunity to allow the clinic or center to decide how to most appropriately use their staff time and resources is critical to maintaining the highest standard of care for their patients. One commenter suggested that, in addition to revising the time frame for review, CMS should also reduce the burden of this regulation by removing the requirement that someone in the group of professional personnel that reviews the policies must be from outside the clinic or center's staff.

Response: We continue to believe these two changes are appropriate, and are finalizing them as proposed.

We agree that the requirement to have someone in the group of professional personnel that reviews the policies be from outside of the clinic or center's own staff can be difficult to meet in medically underserved areas or those where there are health professional shortages. Administrative burden would be decreased by the time often spent trying to find a qualified professional who is not on payroll, but is willing to come in and review RHC policies. We will consider this change for future rulemaking.

Final Rule Action:

1. We are finalizing the proposal to revise the requirement at $\$ 491.9(b)(4)$ requiring $\mathrm{RHCs}$ and $\mathrm{FQHCs}$ to review their patient care policies at least biennially by a group of professional personnel and RHC or FQHC staff.

2. We are finalizing the proposal to revise the requirement at $\S 491.11$ (a) that requires the clinic or center to carry out or arrange for, a biennial evaluation of its total program.

Contact: CAPT Jacqueline Leach, USPHS, 410-786-4282.

12. Emergency Preparedness for Providers and Suppliers

On September 16, 2016, we published a final rule entitled, "Medicare and Medicaid Programs; Emergency Preparedness Requirements for Medicare and Medicaid Participating Providers and Suppliers"' (81 FR 63860), which established national emergency preparedness requirements for Medicare and Medicaid participating providers and suppliers (referred to collectively as "facilities" in the subsequent section) to plan adequately for both natural and man-made disasters and coordinate with Federal, State, tribal, regional, and local emergency preparedness systems. In that final rule, we emphasized the need for facilities to maintain access to healthcare services during emergencies, safeguard human resources, and maintain business continuity and protect physical resources. A facility's emergency preparedness program must include the following elements:

- Risk assessment and emergency planning

- Policies and procedures

- Communication plan

- Training and testing

We received over 300 comments centered around the proposed revisions to the Emergency preparedness requirements. Some of the comments were supportive of one or more of the proposed provisions, others were not supportive of the proposed revisions and expressed the commenters concerns. We have organized our responses to the comments as follows: (1) General Comments; (2) Annual Review of Emergency Preparedness Program; (3) Documentation of Cooperation Efforts; (4) Annual Emergency Preparedness Training Program; (5) Annual Emergency Preparedness Testing.

\section{General Comments}

Comment: One commenter suggested that we delay any changes to emergency preparedness for 5 years. The commenter states that revisions set forth in the September 2016 Emergency Preparedness final rule (81 FR 63860) just went into effect within the past year and some facilities are still working to come into compliance with those changes. Commenters assert that implementing additional revisions at this time would be burdensome.

Response: The September 2016 Emergency Preparedness final rule (81 
FR 63860) was a comprehensive change in our requirements for all provider types. Therefore, we allowed additional time for providers and suppliers to come into compliance. We do not agree that it is necessary to extend the effective date because (1) the original compliance date was 2017, so providers and suppliers should be complete with implementation; (2) the proposed changes in this rule decrease burden, so implementation should not impose a hardship on providers and suppliers to come into compliance. Therefore, we are not delaying the implementation of this requirement. Once this rule is published, providers/suppliers will have 60 days from the publication date to be in compliance with the finalized changes.

Comment: One commenter requested that we leave the emergency preparedness regulations as they are and work instead on strengthening standards as proposed in Sheltering in Danger, a report written by Minority Staff of the Senate Finance Committee. The report discusses efforts to improve nursing home quality by calling attention to specific issues such as heat index/ temperature/humidity, sheltering and evacuations and community engagement.

Response: We appreciate the Committee's work on the Sheltering in Danger report. We updated Appendix Z of the State Operations Manual in February 2019 to clarify the emergency preparedness requirements. This includes adding emerging infectious diseases to the definition of all-hazards approach; clarifications and additional guidance on the use of portable generators and alternate source power and a cross reference to the nursing home requirements for safe temperatures; and technical changes to the home health citations. We are always looking for ways to improve quality and safety oversight efforts in nursing homes, and are continuing to consider the report's recommendations as we move forward.

Comment: A few commenters stated that the current emergency preparedness requirements are overly burdensome for outpatient providers/ suppliers and the requirements should be different for outpatient versus inpatient providers and suppliers. The commenters expressed that providers/ suppliers that provide inpatient services should have stronger requirements as the patients or residents may be incapable of self-preservation in the event of an emergency. Whereas, outpatient providers and suppliers generally have patients that are capable of self-preservation in the event of an emergency.

Response: We understand that for many smaller, rural providers and suppliers and for outpatient facilities that do not have full-time patients the emergency preparedness requirements may seem excessive. Many of the requirements are similar for inpatient and outpatient providers and suppliers. We believe these emergency preparedness requirements are important for all providers and suppliers. However, we recognize that there are some differences in inpatient and outpatient facilities with regard to emergency preparedness and have made changes in this rule that recognize these differences. In addition, we note that LTC facilities have some changes in requirements for the emergency plan updates and training that are discussed in detail below. We will take your recommendation and consider it for future rulemaking.

a. Annual Review of Emergency Preparedness Program ( $\S 403.748$, 416.54, 418.113, 441.184, 460.84, 482.15, 483.73, 483.475, 484.102, 485.68, 485.625, 485.727, 485.920, 486.360, 491.12, and 494.62 (a), (b), (c), and $(\mathrm{d}))$

We proposed to change the requirement for facilities to review their emergency preparedness program at least every 2 years. This would increase the facility's flexibility to review their programs as they determine best fits their needs. We are finalizing this proposal with modifications to LTC facilities only.

The comments received in response to the proposed revision were mostly supportive and the comments that were not supportive were mostly centered around LTC facilities. Below is a summary of the comments we received and our responses.

Comment: Many commenters supported the emergency preparedness updates for biennially revisions to the emergency plan. One commenter stated that annual revisions are not always necessary, as urgent changes are made as needed; otherwise, facilities are reviewing procedures that have not changed. The proposed revisions to emergency preparedness requirements would increase facilities' flexibility to build, train, test and review an effective program that meets the needs of each facility and community in which the facility is located.

Response: We agree that requiring facilities to review their emergency preparedness plan biennially allows for more flexibility for providers and suppliers. We expect that facilities would routinely revise and update their policies and operational procedures to ensure that they are operating based on best practices. In addition, facilities should update their emergency preparedness program more frequently than every 2 years as needed (for example, if staff changes occur or lessons-learned are acquired from a reallife event or exercise). Therefore, we are finalizing this proposal for all providers/suppliers to update their emergency preparedness plan

biennially. As discussed in greater detail below, due to the vulnerability of residents in LTC facilities, we are not finalizing the proposal for those facilities only and will require them to update their emergency plan annually, as is currently required. This will allow the staff and residents to be fully aware of the emergency preparedness program and any changes made.

Comment: As noted above, we received many comments that asked us to not finalize the proposed emergency preparedness requirements for LTC facilities. One commenter stated that ongoing communication and collaboration are very important. The current regulations sensibly require annual updates to emergency plans, policies and procedures, communications plan, training and testing. The success of a preparedness plan often depends on frequent updates. Significant changes can occur in a 2 year period, the resident population, as well as local health care providers, transportation companies, staff, facilities, patient population and other vendors. The LTC facility should know about changes in their community. Staff turnover is a concern and for that reason emergency preparedness plans need to be revisited yearly to be sure everyone is prepared. Many commenters stated that changing the requirements to biennial updates creates additional opportunities for errors and for facility residents and staff to be unprepared, lack appropriate response and endanger more residents' lives. Residents depend heavily on the staff and rely on their preparedness during an emergency. The effort and expense of annual updating is far outweighed by the benefit of a LTC facility being prepared for an emergency. Moving to biennial review could exacerbate the issue of emergency preparedness in LTC facilities more than already exists.

Response: We recognize that LTC facility residents are generally a very vulnerable population that rely on the staff to be knowledgeable and prepared in the event of an emergency. For that reason, we are not finalizing the proposal for biennial updates to the 
emergency plan for LTC facilities only. All other providers and suppliers will be required to update their emergency preparedness plan biennially. We would like to point out that this is the minimum requirement for non-LTC facility providers and suppliers and that non-LTC facility providers and suppliers are encouraged to review and update their facilities plan more frequently if providers and suppliers feel the need to.

b. Documentation of Cooperation Efforts $(\S \S 403.748(\mathrm{a})(4), 416.54(\mathrm{a})(4)$, 418.113(a)(4), 441.184(a)(4), 460.84(a)(4), 482.15(a)(4), 483.73(a)(4), 483.475(a)(4), 484.102(a)(4), 485.68(a)(4), 485.625(a)(4), 485.920(a)(4), 486.360(a)(4), 491.12(a)(4), and 494.62(a)(4))

We proposed to eliminate the requirement that facilities document efforts to contact local, tribal, regional, State, and Federal emergency preparedness officials and facilities' participation in collaborative and cooperative planning efforts. Facilities will still be required to include a process for cooperation and collaboration with local, tribal, regional, State and Federal emergency preparedness officials' efforts to maintain an integrated response during a disaster or emergency situation.

The comments received regarding this proposal were mostly supportive. Below we have summarized the comments received and our responses.

Comment: Many commenters support the elimination of documentation of efforts to contact local, tribal, regional, State and Federal emergency preparedness officials and, when applicable, document the facility's participation in collaborative and cooperative planning efforts. Commenters state that documenting efforts to contact emergency preparedness officials are overly burdensome. The commenters also stated that eliminating this requirement allows for smaller facilities to focus on patient care.

Response: We agree that the documentation requirement can be overly burdensome, as some comments have raised, and are finalizing the proposal to remove the requirement. We believe that eliminating this documentation requirement would reduce burden by not requiring facilities to demonstrate that they have contacted local, tribal, regional, State, and Federal emergency preparedness officials or participated in collaborative and cooperative planning in the community, while still requiring facilities to have a process for cooperation and collaboration. Therefore, we are finalizing this requirement as proposed and eliminating the documentation requirement for collaboration with emergency preparedness officials. Providers and suppliers would still be required to have a process for cooperation and collaboration as part of the emergency plan.

Comment: Commenters stated that removing documentation requirements will reduce transparency of cooperation efforts, increasing the likelihood of disjointed responses and weakening accountability. Documentation proves that the facility has actually contacted and collaborated with EP officials, is the only way a state survey agency can verify that efforts have been made for compliance, and is invaluable to incoming staff.

Response: We would like to point out that providers would still be required at the respective emergency preparedness requirements for each provider and supplier to include a process for collaboration/cooperation with officials; however, they would not be required to document efforts to contact these officials. Therefore, this maintains the existence of a process for collaboration with officials without posing additional documentation burdens. Therefore, we are finalizing this requirement as proposed and eliminating the documentation requirement for collaboration with emergency preparedness officials.

c. Annual Emergency Preparedness Training Program (§§ 403.748(d)(1)(ii), 416.54(d)(1)(ii), 418.113(d)(1)(ii), 441.184(d)(1)(ii), 460.84(d)(1)(ii), 482.15(d)(1)(ii), 483.73(d)(1)(ii), 483.475(d)(1)(ii), 484.102(d)(1)(ii), 485.68(d)(1)(ii), 485.625(d)(1)(ii), 485.727(d)(1)(ii), 485.920(d)(1)(ii), 486.360(d)(1)(ii), 491.12(d)(1)(ii), and 494.62(d)(1)(ii))

Facilities are required to develop and maintain a training program that is based on the facility's emergency plan. This emergency preparedness training must be provided at least annually and a well-organized effective training program must include initial training in emergency preparedness policies and procedures. We revisited the public comments received on the Emergency Preparedness proposed rule (81 FR 63890 through 63891) and determined that requiring facilities to provide annual training may be unduly burdensome. Therefore, we proposed to require facilities to provide training biennially or every 2 years, after facilities conduct initial training on their emergency program. In addition, we proposed to require additional training when the emergency plan is significantly updated.

Overall, the majority of commenters opposed our proposal to require emergency preparedness training biennially. We received a significant number of comments on this proposal from nursing home resident advocates. We received a few supportive and negative comments from other stakeholders, including Congressional representatives and emergency management professionals. A summary of the major issues and our responses are set forth below:

Comment: Nursing home resident advocates overwhelmingly opposed our proposal to require emergency preparedness training biennially. These commenters noted that training every 2 years is not sufficient to maintain readiness in the event of an emergency. Commenters noted that nursing homes specifically experience high staff turnover, changes in ownership, and changes in resident conditions/needs, and cited these conditions as reasons to support annual training. Commenters also noted recent emergency events and the lack of readiness displayed by nursing homes as an indication that more emergency preparedness training, not less, is needed.

In addition to the large number of comments from nursing home resident advocates, we also received a few comments opposed to the proposal from non-LTC facility providers. These commenters also noted high staff turnover, changes in community resources, closure of receiving providers, changes in patient/resident census, and the need to incorporate recent best practices and lessons learned as the main reasons to support annual training. Commenters indicated that the effort and expense of annual training would be outweighed by the benefit of being prepared in the case of an emergency or natural disaster.

Response: We appreciate the feedback and thoughtful comments provided on this proposal. We especially appreciate the comments that provided a very detailed analysis of the lack of emergency response in nursing homes following recent emergency events. We believe that these comments have provided compelling evidence to revise our proposal specific to LTC facilities. Therefore, for LTC facilities only, we are not finalizing our proposal to revise the annual training requirement to biennial training. LTC facilities will be required to continue to meet the current requirement for annual training. 
d. Annual Emergency Preparedness

Testing (§§ 403.748(d)(2), 416.54(d)(2), 418.113(d)(2), 441.184(d)(2),

460.84(d)(2), 482.15(d)(2), 483.73(d)(2), 483.475(d)(2), 484.102(d)(2),

485.68(d)(2), 485.625(d)(2),

485.727(d)(2), 485.920(d)(2), 486.360(d)(2), 491.12(d)(2), and 494.62(d)(2))

Facilities are currently required to conduct exercises to test the emergency plan at least annually. The facility must conduct two emergency preparedness testing exercises every year. Specifically, facilities must:

- Participate in a full-scale exercise that is community-based or when a community-based exercise is not accessible, an individual, facility-based. If the facility experiences an actual natural or-man made emergency that requires activation of the emergency plan (including their communication plan and revision of the plan as needed), the facility is exempt from engaging in a community-based or individual, facility based full-scale exercise for 1 year following the onset of the actual event;

- Conduct an additional exercise that may include either a second full-scale exercise that is community-based or individual, facility-based or a tabletop exercise that includes a group discussion led by a facilitator.

Upon further analysis of this requirement, and taking into account stakeholder feedback, we determined that there was a need to clarify and revise some of the requirements included in the Emergency Preparedness final rule (81 FR 63860). Therefore, for all provider and supplier types, we proposed to clarify our intent with regard to the types of testing exercises, specifically full-scale exercises and functional exercises.

For providers of inpatient services (inpatient hospice facilities, Psychiatric Residential Treatment Facilities (PRTFs), hospitals, long-term care facilities (LTCFs), ICFs/IIDs, and CAHs), we proposed to retain the existing requirement for these provider and supplier types to conduct two emergency preparedness testing exercises annually. We proposed to expand the testing requirement options, such that one of the two annually required testing exercises could be an exercise of their choice, which could include one community-based full-scale exercise (if available), an individual facility-based functional exercise, a drill, or a tabletop exercise or workshop that included a group discussion led by a facilitator. We noted that although RNHCIs provide inpatient services, we determined that changing their existing requirements to make them consistent with this proposed provision would be unduly burdensome, as they are currently only required to conduct a paper-based, tabletop exercise at least annually.

For providers of outpatient services (ASCs, freestanding/home-based hospice, Program for the All-Inclusive Care for the Elderly (PACE), HHAs, CORFs, Organizations (which include Clinics, Rehabilitation Agencies, and Public Health Agencies as Providers of Outpatient Physical Therapy and Speech-Language Pathology Services), CMHCs, Organ Procurement Organizations (OPOs), RHCs, FQHCs, and ESRD facilities), we proposed to require that providers of outpatient services conduct only one testing exercise per year. Furthermore, we proposed to require that these providers participate in either a community-based full-scale exercise (if available) or conduct an individual facility-based functional exercise every other year. In the opposite years, we proposed to allow these providers to conduct the testing exercise of their choice, which may include either a community-based full-scale exercise (if available), an individual, facility-based functional exercise, a drill, or a tabletop exercise or workshop that includes a group discussion led by a facilitator. We noted that due to the nature of services provided by OPOs, we proposed to require that they have the option of providing either a tabletop exercise or workshop every year.

Lastly, we proposed to clarify the testing requirement exemption by noting that if a provider experiences an actual natural or man-made emergency that requires activation of their emergency plan, inpatient and outpatient providers will be exempt from their next required full-scale community-based exercise or individual, facility-based functional exercise following the onset of the actual event.

The majority of the comments received were supportive of our proposal to differentiate the emergency preparedness testing requirements between inpatient and outpatient providers and to clarify the types of testing exercises that will satisfy the proposal. A summary of the major comments and our responses are below:

Comment: While many commenters supported our requirement to differentiate the emergency preparedness testing requirements between inpatient and outpatient providers, one commenter noted that the varying requirements may discourage coordination and collaboration amongst providers within a community.

Response: We appreciate the feedback in support of our proposal. It is not our intention to discourage coordination among providers, but rather to provide facilities with a requirement for emergency preparedness testing that is realistic and attainable, without impacting the health and safety of the patients that they serve. We believe that differentiating the testing requirements by inpatient and outpatient provider and supplier types takes into consideration the unique characteristics of not only the provider type, but also the population that they serve. We expect that facilities will continue to make best efforts to collaborate with providers within their community to not only maximize efforts and resources, but to also meet the many other emergency preparedness requirements for coordination and collaboration. We note that all provider and supplier types are required to develop an emergency preparedness communication plan that, among other things, includes information for other providers; and to develop a method for sharing information and medical documentation for individuals under the provider's care with other health care providers, as necessary to maintain the continuity of care.

Comment: Commenters supported the clarification of the types of testing exercises that would satisfy the testing requirements. However some commenters indicated that the proposal, and terminology we used, remain confusing. These commenters urged us to follow the principles of exercise programs established under the Homeland Security Exercise and Evaluation Program (HSEEP). One commenter indicated that we use functional exercise and full-scale exercise interchangeably, when the two exercises are vastly different types of exercises. This commenter suggested further that we use a more broad definition of the types of testing exercises to align with HSEEP. Specifically, the commenter recommended that we require facilities to participate in an annual operationsbased exercise in conjunction with local, county, or other state stakeholders (if available) or conduct an operationsbased exercise at the facility level. The commenter noted that, as defined by HSEEP, an "operations-based exercise" could include any of the following types of exercises: Drill, functional exercise, or full-scale exercise. Furthermore, the commenter indicated that as a choice of testing exercises we should specify that 
facilities may choose a "discussion based exercise" that, as defined by HSEEP, would include a tabletop exercise or workshop.

Response: We appreciate the feedback and want to ensure that the language used in our regulations and the intent behind our regulations are as clear as possible. As indicated in the proposed rule and as well in the 2016 Emergency Preparedness final rule (81 FR 63860), we have attempted to align our terminology with that used by HSEEP. We note that functional exercise and full-scale exercise are specific testing exercise types as defined by HSEEP. Furthermore, in the proposed rule (83 FR 47714) we provided definitions for both functional and full-scale exercises, as defined by HSEEP. Therefore, we disagree with the commenters who suggested that we have not aligned our proposal with the guiding principles of HSEEP.

It is our intent that providers and suppliers make an attempt to conduct a full-scale exercise within their community, while understanding that this may not always be feasible.

Therefore, we provide that when a fullscale exercise is not available, facilities must conduct a functional exercise at the individual facility level in order to satisfy our requirement. The commenter's suggestion to broaden the language to "operations-based exercise" would mean that a drill could also satisfy our requirement, and that is not our intention. We specifically refer to a full-scale exercise and functional exercise because those are the two testing exercises that would satisfy the requirement. We encourage readers to refer to the proposed rule (83 FR 47714) and the HSEEP guidelines located at https://preptoolkit.fema.gov/ documents/1269813/1269861/HSEEP Revision_Apr13_Final.pdf/65bc78431d10-47b7-bc0d-45118a4d21da for additional details regarding the definition of these types of exercises.

While we have not made any modifications to the terminology used to highlight the testing types, we have reviewed the regulatory text for opportunities to improve readability and have made minor revisions to the regulatory language in hopes of providing clarity about what is required

Final Rule Action:

- We are not finalizing our proposal to require biennial updates to the emergency preparedness program for LTC facilities only. All other affected providers are required to update the emergency preparedness program biennially.

- We are finalizing our proposal to eliminate the requirement that facilities document efforts to contact local, tribal, regional, State, and Federal emergency preparedness officials and facilities' participation in collaborative and cooperative planning efforts.

- We are not finalizing our proposal to require biennial emergency preparedness training for LTC facilities only. All other affected providers are required to provide emergency preparedness training biennially.

- We are finalizing our proposal to require inpatient providers to conduct two testing exercises annually and outpatient providers to conduct one testing exercise annually with only minor modification to improve the readability and clarity of the requirement.

Contact: Kristin Shifflett, 410-7864133, Ronisha Blackstone, 410-7866882 .

\section{Technical Corrections}

In response to public comments, we are revising that language used to reference doctors of dental surgery that appear in the regulatory text for hospitals. The hospital CoPs reference these physicians in the Medical Staff CoP (§§ 482.22(c)(5)(1) and 482.22(c)(6)) as oromaxillofacial surgeons. The accurate and current terminology to use for these physicians is oral and maxillofacial surgeons. We are revising the regulatory text for these provisions.

Although we did not propose this in the proposed rule, in response to public comments regarding home health aide competency training, we are revising the language used to describe the process for conducting home health aide competency evaluations to restore longstanding official CMS policy. In the July 18, 1991 (56 FR 32967) final rule, "Medicare Program; Home Health Agencies: Conditions of Participation," issued by CMS, we explicitly permitted the use of pseudo-patients and laboratory environments for purposes of home health aide competence evaluations, even though the regulatory text did not specifically mention "pseudo-patients." We stated, “[W]e believe that it is acceptable to conduct aide training with a mannequin and to conduct competency evaluations in a laboratory setting using 'pseudo patients' such as another aide or volunteer. We do not believe it is necessary to revise the regulations to clarify this point." (56 FR 32972). We agree with commenters that it is necessary to make a technical correction to the HHA CoPs as finalized on January 13, 2017 (82 FR 4584) to explicitly permit the use of pseudo-patients for purposes of home health aide competency evaluations in order to assure that the home health agency regulations and Interpretive Guidelines are consistent with the policy originally set forth in 1991.

This technical correction restores longstanding CMS policy, as stated in the 1991 rule, that permitted the use of pseudo-patients, and is consistent with the original intent of the January 2017 HHA CoPs final rule. We are making conforming changes to the definitions section of the HHA CoPs at $\S 484.2$ to define the terms "pseudo-patient" and "simulation" as follows:

- "Pseudo patient means a person trained to participate in a role-play situation, or a computer-based mannequin device. A pseudo-patient must be capable of responding to and interacting with the home health aide trainee, and must demonstrate the general characteristic to the primary patient population served by the HHA in key areas such as age, frailty, functional status, and cognitive status.'

- "Simulation means a training and assessment technique that mimics the reality of the homecare environment, including environmental distractions and constraints that evoke or replicate substantial aspects of the real world in a fully interactive fashion, in order to teach and assess proficiency in performing skills, and to promote decision making and critical thinking."

Because this is a clarification of an already-existing rule to codify longstanding policy, we do not believe that notice and comment rulemaking is necessary; we are therefore waiving notice and comment as indicated in Section I.C.14 below.

\section{General Comments}

Comment: We received many comments regarding issues that are out of scope of this rule, such as payment and reimbursement, Medicare advantage, prior authorization, physical therapy requirements and more. Some of these issues were for specific

providers or suppliers and some were blanket comments.

Response: We have read and received all of the comments that are out of the scope of this rule. We will not be addressing them in this rule; however, we will consider them for future rulemaking.

\section{Waiver of Proposed Rulemaking}

We ordinarily publish a notice of proposed rulemaking in the Federal Register and invite public comment on the proposed rule. The notice of proposed rulemaking includes a reference to the legal authority under which the rule is proposed, and the terms and substances of the proposed 
rule or a description of the subjects and issues involved. This procedure can be waived, however, if an agency finds good cause that a notice-and-comment procedure is impracticable, unnecessary, or contrary to the public interest and incorporates a statement of the finding and its reasons in the rule issued.

A notice-and-comment rulemaking procedure is unnecessary for the change related to adding the phrase "or with a pseudo-patient as part of a simulation" to the HHA aide competency evaluation requirement at $\$ 484.80$ (c)(1) because this regulatory revision simply restores official CMS policy as stated in rulemaking dating back to 1991, and does not constitute a change in CMS policy. We are adding conforming changes to the definitions section at $\S 484.2$ for the terms "pseudo-patient" and "simulation." These changes are technical in nature. These changes to restore longstanding CMS policies are in the public interest, in order to assure that HHAs are adequately staffed with aides that have proven their competency to serve HHA patients. Home health aides may not provide services to patients until they have demonstrated their skill competencies. Allowing HHAs to use pseudo-patients as part of a simulation in order to demonstrate skill competencies facilitates timely placement of properly trained and evaluated aides in patient homes to provide much needed services in accordance with each patient's individualized plan of care. In the absence of this regulatory change to conform to longstanding CMS policy, in a survey conducted by the National Home Care Association 45 percent of responding HHAs reported being unable to provide full competency examinations for newly hired home health aides, creating a delay in delivering physician-ordered aide services to HHA patients. This delay in direct patient care services may be harmful to patients, and the technical change will resolve the underlying aide competency evaluation backlog problem that is creating the delay.

Therefore, we find good cause to waive the notice of proposed rulemaking and to issue these provisions on an interim basis. We are providing a 60-day public comment period.

\section{Collection of Information Requirements}

Under the Paperwork Reduction Act of 1995 (PRA), we are required to provide 30-day notice in the Federal Register and solicit public comment before a collection of information requirement is submitted to the Office of
Management and Budget (OMB) for review and approval. In order to fairly evaluate whether an information collection should be approved by OMB, section 3506(c)(2)(A) of the PRA requires that we solicit comment on the following issues:

- The need for the information collection and its usefulness in carrying out the proper functions of our agency.

- The accuracy of our estimate of the information collection burden.

- The quality, utility, and clarity of the information to be collected.

- Recommendations to minimize the information collection burden on the affected public, including automated collection techniques.

We solicited public comment on each of the section 3506(c)(2)(A)-required issues for the following information collection requirements (ICRs).

\section{Wage Costs}

To derive average costs, we used data from the U.S. Bureau of Labor Statistics' May 2017 National Occupational Employment and Wage Estimates for all salary estimates (https://www.bls.gov/ oes/2017/may/oes_nat.htm). In this regard, the following table presents the mean hourly wage, the cost of fringe benefits and overhead costs (calculated at 100 percent of salary), and the adjusted hourly wage cost.

TABle 2-National Occupational EMPloyment AND WAGE Estimates

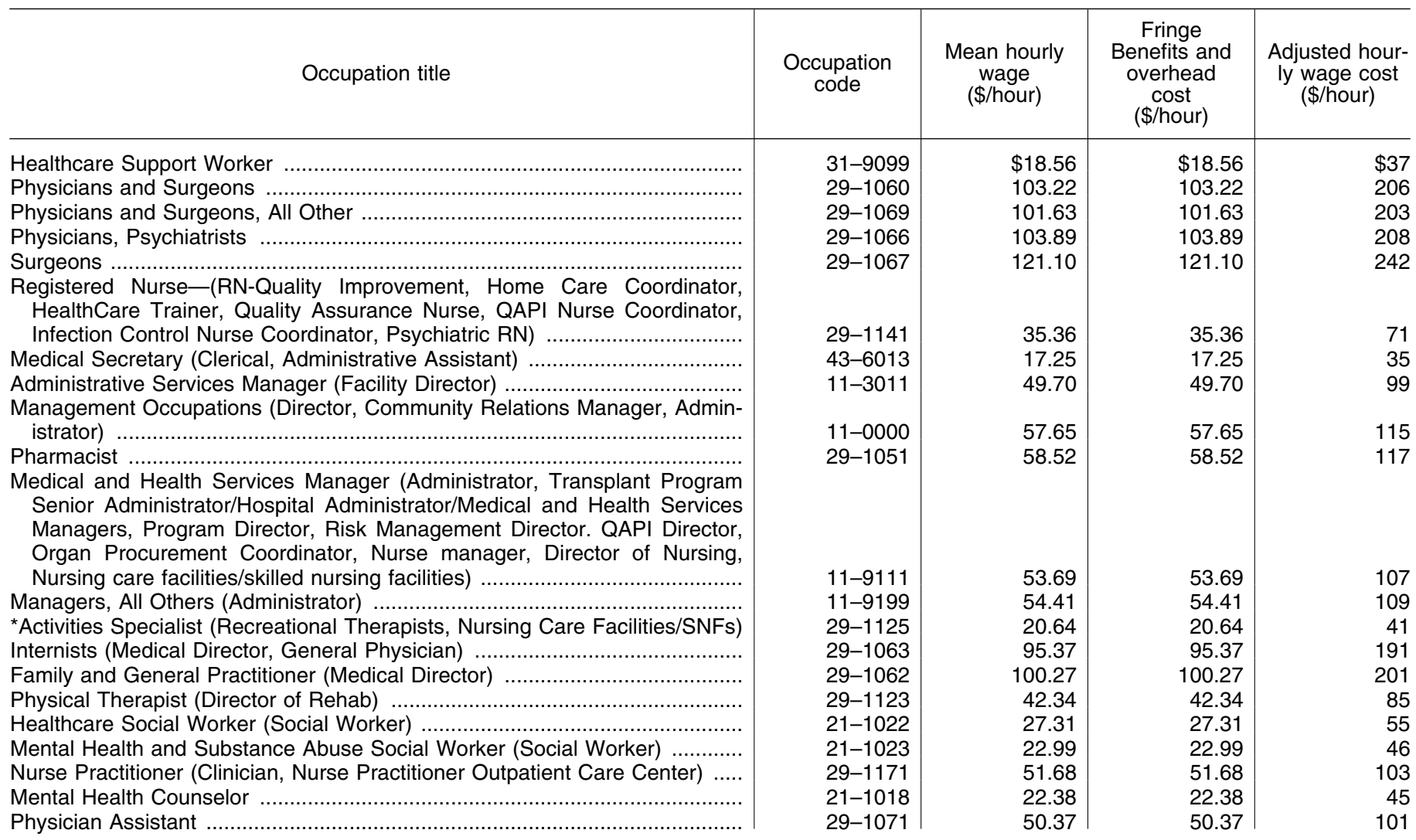


TABle 2-NATIONAL Occupational EMPLOYMent AND WAGE Estimates—Continued

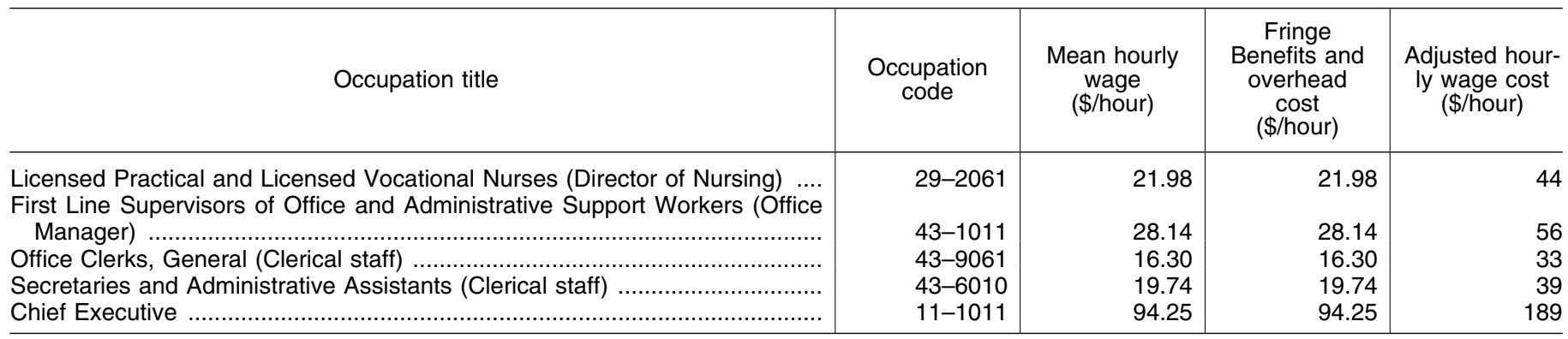

* Salary information used is for Nursing Care Facility/SNF industry.

\section{ICRs Regarding RNHCI Discharge Planning (§ 403.736(a) and (b))}

It was discovered during review that the burden for existing requirements at 42 CFR 403.724(a), 403.730(a), 403.732, 403.736(a)(b), and 403.736(d) was erroneously not accounted for nor approved under the PRA prior to this rulemaking. Accordingly, the burden associated with these requirements is currently pending OMB approval (OMB control number 0938-NEW). Section 403.736 will reduce the extensive requirements for an RNHCI to coordinate with other medical providers for post-RNHCI care. Based on recent claims data, there was a combined annual total of 619 beneficiaries that stayed in the 18 facilities.

We estimate that the time currently required to develop and document discharge plans and activities is 1,238 burden hours (2 hours for each of the 619 beneficiaries discharged) and that it would be reduced by half. Of the approximately 619 annual discharges, we estimate that a RNHCIs burden would be reduced to one hour for each discharged individual. A RNHCI would not need to develop a discharge plan that includes medical care once a patient leaves the RNHCI because doing so would not be in keeping with the religious tenets of the patients they serve. We estimate that the healthcare support worker responsible for a patients discharge plan costs \$37 an hour, including hourly wage and an estimated 100 percent add-on for fringe benefit costs and overhead costs (this is an HHS standard calculation). Based on our experience with RNHCIs, we estimate that it would take 1 hour to develop the proposed discharge instructions and discuss them with the patient or caregiver. We estimate a total of 619 annual discharges from RNHCIs at a savings of $\$ 37$ per discharge for a total savings of $\$ 22,903(\$ 37 \times 619$ hours).
3. ICRs Regarding ASC Governing Body and Management (§ 416.41(b)(3)(i) and (ii))

We are finalizing our proposal with changes to eliminate the requirements at $\S 416.41$ (b)(3) that states the ASC must have a written transfer agreement with a hospital or ensure all physicians performing surgery in the ASC have admitting privileges at a local hospital that meets CMS hospitalization requirements. However, we will require that the ASCs have a notice requirement with hospitals and encourage a transfer agreement when possible. All ASCs easily meet this requirement and have established a relationship with their local hospital and obtained an agreement as usual and customary practice for running an ASC, with the exception of approximately twenty ASCs that have difficult relationships with their local hospitals. The savings would not be significant, however, it does affect the 20 ASCs by removing the requirement. The current information collection request for the ASC rules (OMB control number 0938-1071) does not address any potential burden associated with this requirement. We believe that having and maintaining written agreements is standard practice. Therefore, removing this requirement would not alter the current information collection burden for ASCs.

\section{ICR Regarding ASC Medical Records} $(\S 416.47(\mathrm{~b})(2))$

We are finalizing our proposal to revise $\S 416.47(\mathrm{~b})(2)$ by adding the phrase "(as applicable)" to the significant medical history and results of physical examination requirement of documents that must be included in the medical record in order to conform to the changes that we proposed to the mandatory medical history and physical examination requirement. There are no collection of information requirements associated with this proposed change because maintaining a medical record for each patient is a usual and customary practice in accordance with the implementing regulations of the PRA at 5 CFR 1320.3(b)(2).

5. ICRs Regarding ASC Patient Admission, Assessment and Discharge (§ 416.52(a)(1), (2), (3) and (4))

At $\S 416.52$ we are finalizing our proposal to replace the requirement that every patient have a comprehensive medical history and physical examination (H\&P) within 30 days prior to surgery in an ASC with a requirement that allows the operating physician and ASC to determine which patients would require more extensive testing and assessment prior to surgery. The burden associated with this requirement would be the time and effort necessary to create new policies for when, and whether, to require some form of history and physical that would require preoperative examination and testing, and on what time schedule. The current information collection request for the ASC rules (OMB control number 09381071) does not account for any information collection related burden associated with the comprehensive H\&P requirement. We assume that creating these policies (which could leave such decisions to the surgeon's discretion in most or all cases) would require 10 hours of physician time, 10 hours of RN time, and 10 hours of clerical time, at the preceding hourly rates, for a total of 30 hours per facility. This would be a one-time cost of $\$ 3,460$ per facility ([10 $\times \$ 242]+[10 \times \$ 71]+[10 \times \$ 33])$, and $\$ 19.2$ million for all 5,557 facilities. Therefore, this proposed requirement would increase the information collection related burden by $\$ 19.2$ million and 166,710 hours (30 hours $\times$ 5,557 facilities) on a one-time basis for all ASCs.

6. ICRs Regarding Hospice Aide and Homemaker Services (§ 418.76)

At $\S 418.76$ (a) we are finalizing our proposal to defer to State training and competency requirements, where they exist, for hospice aides. The information 
collection request for the hospice requirements $(\mathrm{OMB}$ control number 0938-1067) estimates that a hospice would spend 5 minutes per newly hired hospice aide to document verification that an aide meets the required training and competency requirements, for a total of 372 annual burden hours for all hospices at a cost of $\$ 11,540$. This change to the actual training and competency requirements would not alter the requirement to document the fact that a hospice aide meets one of the training and competency requirements set forth in the rule; therefore there would be no change to the existing collection of information estimates because the estimates relate to the unchanged documentation requirements rather than the actual training and competency requirements that would be revised by this change.

7. ICRs Regarding Drugs and Biologicals, Medical Supplies, and Durable Medical Equipment (§ 418.106(a))

At $\S 418.106$ (a) we are finalizing our proposal to remove the requirement that a hospice ensure that the interdisciplinary group confers with an individual with education and training in drug management as defined in hospice policies and procedures and State law, who is an employee of or under contract with the hospice to ensure that drugs and biologicals meet each patient's needs. The information collection request for the hospice requirements (OMB control number 0938-1067) states that the burden associated with this requirement is the time necessary to document the results of this consultation in each patient's clinical record. In the information collection request we assumed that an average hospice would confer with a pharmacist, and that the pharmacist would document the results of his or her consultation. We estimated that it requires 5 minutes to document the initial review of a patient's drug and biologicals. Additionally, we estimated that it requires 5 minutes of the pharmacist's time to document a review of updates to the patient's drug profile. Based on a 17 day median length of service, we assumed that each patient would likely receive one update to their plans of care. At an average hourly rate of \$117 for a pharmacist, we estimated that it would cost a hospice $\$ 19.50$ per patient $(\$ 117 \times[5$ minutes for initial + 5 minutes for 1 update]) and an annual cost of $\$ 6,942(\$ 19.50 \times 356$ patients). The total annual burden hours for all hospices was estimated to be 264,588 hours $(1,587,527$ patients $\times .1666$ hour per patient), and the total annual burden cost for all hospices (taking into account new wage data) is estimated to be $\$ 30,956,777$ ( $\$ 19.50$ per patient $\times$ $1,587,527$ patients). Therefore, removing the requirement that a hospice must ensure that the interdisciplinary group confers with an individual with education and training in drug management would result in a burden reduction of 264,588 hours and $\$ 30,956,777$.

The information collection request will be revised and sent to OMB.

H. ICRs Regarding Hospices That Provide Hospice Care to Residents of a SNF/NF or ICF/IID (§ 418.112(c)(10) and (f))

At $\S 418.112$ (f) we are finalizing a requirement to allow hospices and long term care facilities the additional flexibility to negotiate the format and schedule for orienting long term care facility staff regarding certain hospicespecific information. This change does not effect the existing hospice information collection request (OMB control number 0938-1067).

9. ICRs Regarding Hospital Quality Assessment and Performance Improvement (QAPI) Program (§ 482.21)

We are finalizing the proposed new standard at $\S 482.21$ (f), "Unified and integrated QAPI program for multihospital systems". We would allow that for a hospital that is part of a hospital system consisting of two or more separately certified hospitals subject to a system governing body legally responsible for the conduct of each hospital, the system governing body could elect to have a unified and integrated QAPI program for all of its member hospitals after determining that such a decision is in accordance with all applicable State and local laws.

As stated in the information collection request for the hospital requirements (expired OMB control number 0938-0328), we estimate that the burden associated with updating and, in some instances, writing new hospital policies directly related to patient care would be an average of eight (8) hours annually for each member of hospital staff involved in the specific patient care policies addressed.

Patient care policy development (and revision) by hospital medical staff is essential to patient health and safety because it provides the framework within which all patient care services are furnished. Thus, we have included the involvement of a physician at approximately $\$ 1,624$ annually (8 burden hours $\times \$ 203$ ), a QAPI nurse coordinator at $\$ 568$ annually (8 burden hours $\times \$ 71$ ), and a medical secretary at $\$ 280$ annually (8 burden hours $\times \$ 35$ ).
We estimate the necessary policy changes needed to comply with the requirements proposed in this rule would cost $\$ 2,472$ per year $(\$ 1,624+$ $\$ 568+\$ 280$ ) for each of the 424 hospital systems that would be eligible to do so and that would choose to exercise this option. Therefore, the total annual cost for all eligible hospital systems to meet these information collection requirements would be approximately \$1 million.

10. ICRs Regarding Hospital Medical Staff, Medical Records Services, and Surgical Services (§§ 482.22, 482.24, and 482.51)

At $\S 416.52$, we are finalizing our proposal to replace the requirement that every patient have a comprehensive $\mathrm{H} \& \mathrm{P}$ within 30 days prior to surgery in an ASC with a requirement that allows the operating physician and ASC to determine which patients would require more extensive testing and assessment prior to surgery. As discussed in "Provisions of the Proposed Regulations," section II.D.2 of the proposed rule, there is a similar regulatory requirement for hospital outpatient surgery. Based on the substantial similarity between these two service settings, we proposed, through the revisions to $\S \S 482.22,482.24$, and 482.51 discussed in section II.D.2, to provide an exception to these requirements for outpatient surgery in hospitals.

As stated in the information collection request for the hospital requirements (expired OMB control number 0938-0328), which is in the process of being reinstated, we estimate that the burden associated with updating and, in some instances, writing new hospital policies directly related to patient care would be an average of eight (8) hours annually for each member of hospital staff involved in the specific patient care policies addressed.

Patient care policy development (and revision) by hospital medical staff is essential to patient health and safety because it provides the framework within which all patient care services are furnished. Thus, we have included the involvement of a physician at approximately $\$ 1,624$ annually (8 burden hours $\times \$ 203$ ), a nurse coordinator at $\$ 568$ annually (8 burden hours $\times \$ 71$ ), and a medical secretary at $\$ 280$ annually (8 burden hours $\times \$ 35$ ).

We estimate that the necessary policy changes needed to comply with the requirements in this rule would cost $\$ 2,472$ per year $(\$ 1,624+\$ 568+\$ 280)$ for each of the 4,823 hospitals that might choose to exercise this option. 
Therefore, the total annual cost for all hospitals to meet these information collection requirements would be approximately $\$ 11.9$ million.

11. ICRs Regarding Hospital Medical Staff: Autopsies (§ 482.22)(d))

We are finalizing our proposal to remove the requirement at $\$ 482.22(\mathrm{~d})$, which states that a hospital's medical staff should attempt to secure autopsies in all cases of unusual deaths and of medical-legal and educational interest. Hospitals are further required to define a mechanism for documenting permission to perform an autopsy, and they must have a system for notifying the medical staff, and specifically the attending practitioner, when an autopsy is being performed. Since more detailed, specific requirements regarding medical-legal investigations and autopsies for hospitals are covered by the individual State laws in which the hospital is located, there are no collection of information requirements associated with this proposed change.

12. ICRs Regarding Hospital Infection Control (§ 482.42)

We are finalizing the proposed new standard at § 482.42(d), "Unified and integrated infection control program for multi-hospital systems." Like the proposed requirements for a unified and integrated QAPI program, the proposed standard for infection control would allow that for a hospital that is part of a hospital system consisting of multiple separately certified hospitals subject to a system governing body legally responsible for the conduct of each hospital, such system governing body could elect to have a unified and integrated infection control program for all of its member hospitals after determining that such a decision was in accordance with all applicable State and local laws.

As stated in the information collection request for the hospital requirements $(\mathrm{OMB}$ control number 0938-0328), which is in the process of being reinstated, we estimate that the burden associated with updating and, in some instances, writing new hospital policies directly related to patient care would be an average of eight (8) hours annually for each member of hospital staff involved in the specific patient care policies addressed.

Patient care policy development (and revision) by hospital medical staff is essential to patient health and safety because it provides the framework within which all patient care services are furnished. Thus, we have included the involvement of a physician at approximately $\$ 1,624$ annually (8 burden hours $\times \$ 203$ ), an infection control nurse coordinator at $\$ 568$ annually (8 burden hours $\times \$ 71$ ), and a medical secretary at $\$ 280$ annually (8 burden hours $\times \$ 35$ ).

We estimate the necessary policy changes needed to comply with the requirements proposed in this rule would cost $\$ 2,472$ per year $(\$ 1,624+$ $\$ 568+\$ 280$ ) for each of the 424 hospital systems that would be eligible to do so and that would elect to exercise this option. Therefore, the total annual cost for all eligible hospital systems to meet these information collection requirements would be approximately $\$ 1$ million.

\section{ICRs Regarding Special}

Requirements for Hospital Providers of Long-Term Care Services ("SwingBeds”) (§ 482.58(b)(1), (4), (5), and (8), and Parallel CAH Requirements: $\S 485.645(\mathrm{~d})(1),(4),(5)$, and (8))

At $\S \S 482.58(b)(1)$ and 485.645(d)(1) (cross-referenced long-term care requirement at $\S 483.10(f)(9)$ ) we are finalizing our proposal to remove the requirement for hospital and $\mathrm{CAH}$ swing-bed providers to provide the right for patients to choose to or refuse to perform services for the facility and if they so choose; (a) document in the resident's plan of care, (b) noting whether the services are voluntary or paid and (c) provide wages for the work being performed given the location quality, and quantity of work requiring comparable skills.

We assume that each of the hospital swing-bed providers (478 hospitals) and CAH swing-bed providers (1,246 CAHs) has an activities specialist employed at $\$ 41$ per hour who would oversee the residents who have chosen to perform services for the facility, and document and update the plan of care accordingly. We believe that given the limited budget of most rural providers, services are being provided to the CAH on a voluntary basis and that these providers are not compensating patients for providing these services. The current regulatory burden for compliance with this requirement is approximately $\$ 29.4$ million for all hospital and CAH swingbed providers, or $\$ 17,056$ per hospital or CAH swing-bed provider $(1,724$ hospital and CAH swing-bed providers $\times \$ 41$ an hour for an activities specialist $\times 8$ hours per week $\times 52$ weeks per year), which are the cost savings to the providers as a result of the removal of this requirement.

At $\S 482.58(b)$ (4) (and $\S 485.645(d)(4)$ ) (cross-referenced long-term care requirement at $\S 483.24$ (c)), we are finalizing our proposal to remove the requirement for hospital and $\mathrm{CAH}$ swing-bed providers to provide an ongoing activity program that is directed by a qualified therapeutic recreation specialist or an activities professional who meets certain requirements as listed at $\S 483.24$ (c)(2). We assume that each of the hospital swing-bed providers (478 hospitals) and CAH swing-bed providers (1,246 CAHs) has an activities specialist employed at least part time at $\$ 41$ per hour. The current regulatory burden for compliance with this requirement is based on the activities specialist organizing, overseeing, and scheduling the activity. The cost savings as a result of the removal of this requirement are approximately $\$ 73.5$ million for all hospital and CAH swingbed providers, or $\$ 42,640$ per hospital or CAH swing-bed provider $(1,724$ hospital and CAH swing-bed providers $\times \$ 41$ an hour for an activities specialist $\times 1,040$ hours per year) which are the cost savings to the providers.

We are finalizing our proposal to remove the requirement at $\S \S 482.58(\mathrm{~b})(5)$ and 485.645(d)(5) (crossreferenced long-term care requirement at $\S 483.70$ (p) for hospital and $\mathrm{CAH}$ swing-bed providers to employ a qualified social worker on a full-time basis if the facility has more than 120 beds. Given that this provision is not applicable to either provider type due to the regulatory requirements for each, it does not impose a burden upon hospitals and as such, its removal would not result in a savings of economic burden hours or dollars.

At $\S \S 482.58(b)(8)$ and 485.645(d)(8)

(cross-referenced long-term care

requirement at $\S 483.55(a)(1)$ ) we are finalizing our proposal to remove the requirement for hospital and $\mathrm{CAH}$ swing-bed providers to assist in obtaining routine and 24-hour emergency dental care to its residents.

Under the current CoPs, hospitals and CAHs are currently required to address the emergent dental care needs of their patients at $\S 482.12(f)(2)$ for hospitals, and at $\S 485.618$ (emergency services) for CAHs. As a result, we have calculated the burden associated with the provision of routine dental care for hospital and swing-bed patients. The American Dental Association recommends annual dental checkups for routine dental care for adults over 60 years of age. With an average length of stay in a hospital or CAH swing-bed of 1-2 weeks and an average daily census of 2 patients, we assume that 1 patient receiving swing-bed services will require routine dental services per month. While a dentist and dental hygienist provide the dental services, Medicare is billed for the provision of these services. The costs to the provider 
are related to the nursing activities associated with the patient receiving the dental services. The current regulatory burden for compliance with this requirement is approximately $\$ 2.9$ million for all hospital and CAH swingbed providers, or $\$ 1,704$ per hospital or CAH swing-bed provider (1,724 hospital and $\mathrm{CAH}$ swing-bed providers $\times \$ 71$ an hour for a $\mathrm{RN} \times 24$ hours per year), which are the cost savings to the providers as a result of the removal of this requirement. The information collection requests will be revised and sent to OMB for approval (OMB control number 0938-0328 for hospitals and 0938-1043 for CAHs).

\section{ICRs Regarding Special}

Requirements for Psychiatric Hospitals (§ 482.61(d))

At $\S 482.61(d)$ we are finalizing our proposal to clarify the requirement allowing non-physician practitioners to document progress notes in accordance with State laws and scope of practice requirements. In accordance with the information collection request for the hospital requirements, which includes the special requirements for psychiatric hospitals (OMB control number 09380328), no burden is associated with recordkeeping, as the documentation and maintenance of medical records is usual and customary. However, since we believe that clarification of the intent of the regulation is necessary and will result in non-physician practitioners (specifically physician assistants, nurse practitioners, psychologists, and clinical nurse specialists) documenting in the progress notes for patients receiving services in psychiatric hospitals, we have calculated savings for this provision in the RIA which are essentially identical to those we would estimate under the PRA.

15. ICRs Regarding Special Requirement for Transplant Centers and Definitions (§§ 482.68 and 482.70)

We are finalizing the proposed nomenclature change at part 482 and the transplant center regulations at $\S \S 482.68,482.70,482.72$ through 482.104 , and at $\S 488.61$. Because this change would update the terminology used in the regulations to conform to the terminology that is widely used and understood within the transplant community, there are no collection of information requirements associated with this proposal.

16. ICRs Regarding Data Submission, Clinical Experience, and Outcome Requirements for Re-Approval of Transplant Centers (§ 482.82)

Section 482.82 requires that, except as specified in $\S 488.61$, transplant centers must meet all the data submission, clinical experience, and outcome requirements to be re-approved for Medicare participation. Section 482.82(a) requires that no later than 90 days after the due date established by the OPTN, a transplant center must submit to the OPTN at least 95 percent of the required data submissions on all transplants (deceased and living donors) it has performed over the 3 year approval period. Furthermore, $\S 482.82$ (b) requires transplant centers to perform an average of 10 transplants per year during the prior 3 years and $\S 482.82$ (c) requires transplant centers to meet the outcome requirements for Medicare re-approval. The burden associated with this requirement would be the time it would take a transplant program to submit the required information (OMB control number 0938-1069). However, as required by $\S \S 482.72$ and $482.45(\mathrm{~b})$, a hospital in which a transplant program is located, must belong to the OPTN, and the OPTN requires that these hospitals submit this data to the OPTN. Therefore, we believe that the requirements under $\S 482.82$ do not impose an additional burden on transplant programs because all Medicare participating transplant programs are already submitting this information to the OPTN. Removing these requirements will have no effect on the collection of information burden on transplant programs.

17. ICRs Regarding Special Procedures for Approval and Re-Approval of Organ Transplant Centers (§ 488.61(f) Through (h))

Section 488.61(f) through (h) sets out the process for our consideration of a transplant center's mitigating factors in initial approval and re-approval surveys, certifications, and enforcement actions for transplant centers. The provisions also set out definitions and rules for transplant systems improvement agreements. We are finalizing our proposal to remove the requirements at $\S 488.61$ (f) through (h) for mitigating factors and transplant systems improvement agreements for the re-approval process for transplant centers. This change is complementary to the removal of $\S 482.82$, described previously. The information collection request (OMB control number 09381069) does not account for any information collection related burden associated with the requirements in $\S 488.61$ (f) through (h) for the reapproval process. Therefore, we estimate that the requirements under $\S 488.61(f)$ would require a transplant program to write and submit the initial formal notice of the program's intent to seek mitigating factors re-approval, and write and submit a request for consideration of mitigating factors (which would include all of the content listed in $\S 488.61(f)(2)$ ). We estimate that this would take a medical director, a transplant center senior administrator, and a hospital administrator approximately 5 hours, or 2 hours for the medical director and the transplant program senior administrator and 1 hour for the hospital administrator, to complete and submit these mitigating factors for re-approval, as described in Table 3.

table 3-Annual Burden Hours and Cost for Transplant Programs to Submit Mitigating Factors for ReAPPROVAL

\begin{tabular}{|c|c|c|c|}
\hline Position & Hourly & $\begin{array}{c}\text { Hours } \\
\text { required }\end{array}$ & $\begin{array}{c}\text { Total } \\
\text { cost estimate }\end{array}$ \\
\hline Medical Director & $\$ 191$ & 2 & $\$ 382$ \\
\hline Transplant Program Senior Administrator & 107 & 2 & 214 \\
\hline Totals & ......... & 5 & 703 \\
\hline
\end{tabular}

In total, we estimate that an average of 14 programs would submit mitigating factors annually. Thus, for those 14 programs we estimate that it would require 70 burden hours ( 5 burden hours $\times 14$ programs) at a cost of $\$ 9,842$ 
$(\$ 703 \times 14$ programs). Removing this requirement would yield an estimated savings to transplant programs of 5 burden hours each and a total of 70 burden hours for all 14 programs, with a total cost savings of $\$ 9,842$.

In addition, we estimate that the transplant hospital in conjunction with the transplant program that is located in the hospital, would submit mitigating factors and then would also enter into systems improvement agreements, as described under § 488.61(h) annually. This would require the hospital to enter into a binding agreement with CMS to allow the program additional time to achieve compliance with the CoPs. We estimate that this would take a medical director, a transplant program senior administrator, a hospital administrator, and an administrative assistant approximately 14 hours, or 4 hours for the medical director, transplant program senior administrator, and an administrative assistant, and 2 hours for the hospital administrator to complete these activities (including notifying patients about the degree of noncompliance by mail and organizing and completing the other tasks listed in $\S 488.61(\mathrm{~h})(1)$ as required by the terms in the systems improvement agreement), as described in Table 4.

Table 4-Annual Burden hours and Cost for Transplant Programs To Enter Into a Systems Improvement AGREEMENT FOR RE-APPROVAL

\begin{tabular}{|c|c|c|c|}
\hline Position & Hourly & $\begin{array}{l}\text { Hours } \\
\text { required }\end{array}$ & $\begin{array}{c}\text { Total } \\
\text { cost estimate }\end{array}$ \\
\hline Medical Director ...................... & $\$ 191$ & 4 & $\$ 764$ \\
\hline Transplant Program Senior Administrator & 107 & 4 & 428 \\
\hline 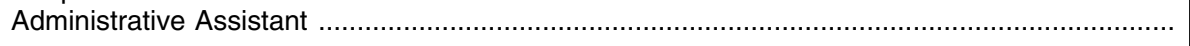 & 35 & 4 & 140 \\
\hline Totals ........... & ............. & 14 & 1,546 \\
\hline
\end{tabular}

In total, we estimate that an average of 14 programs will submit mitigating factors annually. Thus, for those 14 programs we estimate that it would require 196 burden hours (14 burden hours $\times 14$ programs) at a cost of $\$ 21,644$ (\$1,546 $\times 14$ transplant programs). In the context of the proposed rule, removing this requirement would yield an estimated savings to transplant programs of 14 burden hours each and a total of 196 burden hours for all 14 programs, with a total cost savings of $\$ 21,644$.

18. ICRs Regarding HHA Home Health Aide Services (§ 484.80(h)(3))

We are finalizing the proposal to eliminate the requirement at $\S 484.80(\mathrm{~h})(3)$ that the HHA conduct a full competency evaluation of deficient home health aides, and replace it with a requirement to retrain the aide regarding the identified deficient skill(s) and require the aide to complete a competency evaluation related only to those skills. We are also finalizing a change to permit HHAs to use either patients or pseudo-patients when conducting competency evaluations for home health aides. The content of an aide competency examination and whether patients or pseudo-patients are used in the process do not have an associated collection of information requirement. Therefore, this proposed change would neither impose nor remove any collection of information burdens.

19. ICRs Regarding HHA Clinical Records (§ 484.110(e))

As discussed in section I.B.6.c. of this final rule, we are not finalizing the proposal to allow HHAs 4 business days to provide patients with information from their clinical records upon request. Consequently, there are no new information collection requirements.

20. ICRs Regarding CORF Utilization Review Plan (§ 485.66)

We are finalizing the proposal to reduce the required frequency in which
CORFs would be required to complete a "utilization review plan" from quarterly to annually. Changing from a quarterly implementation of the utilization review plan to an annual implementation would reduce the current documentation requirements (OMB control number 0938-1091) on CORFs by 75 percent each year. For the purposes of our analysis, we estimate that it would take a CORF approximately 8 hours for administrative, clinical and clerical staff to review and evaluate the necessary and efficient use of services provided by the facility on a quarterly basis, for a total of 32 hours per year per CORF and 6,016 hours for all 188 CORFs. In a 1year period, we estimate a savings of $\$ 1,680$ per facility ( $\$ 560 \times 3$ quarters), and a combined total savings of $\$ 315,840$ for all CORFs $(\$ 1,680 \times 188$ CORFs). We will submit the revised information collection request to $\mathrm{OMB}$ for approval.

TABLE 5-CORF-HOURLY COSTS AND BURDEN HOURS

\begin{tabular}{|c|c|c|c|}
\hline Position & $\begin{array}{l}\text { Hourly costs } \\
\text { per CORF }\end{array}$ & $\begin{array}{l}\text { Burden hours } \\
\text { per CORF }\end{array}$ & $\begin{array}{l}\text { Cost estimate } \\
\text { per CORF }\end{array}$ \\
\hline Administrator & $\$ 107$ & 2 & $\$ 214$ \\
\hline Clerical Staff & 33 & 2 & 66 \\
\hline Social Worker & 55 & 2 & 110 \\
\hline Total & $\ldots .$. & 8 & 560 \\
\hline
\end{tabular}


21. ICRs Regarding CAH Organizational Structure (\$ 485.627(b)(1))

As of 2017, there were approximately 1,353 CAHs that were certified by Medicare. We are finalizing our proposal for revision of the CAH disclosure requirements imposed on CAHs removes the requirement for CAHs to disclose to CMS its owners, or those with a controlling interest in the $\mathrm{CAH}$ or in any subcontractor in which the CAH directly or indirectly has a 5 percent or more ownership interest, in accordance with 42 CFR part 420 , subpart C (OMB control number 09381043). While we estimate that these changes occur at 2 CAHs per year on average between all 1,353 CAHs, with the vast majority not experiencing any such changes throughout the lifetime of the $\mathrm{CAH}$, each CAH is still required to review the duplicative documentation.
As discussed in our rule, Medicare Program; Criteria and Standards for Evaluating Regional Durable Medical Equipment, Prosthetics, Orthotics and Supplies (DMEPOS); Final Rule and Request for Comments (57 FR 2790, June 18, 1992), the burden associated with this requirement is 1-hour per facility. As a result, this will save all CAHs an estimated $\$ 144,771$ and will save each CAH $\$ 107$ (1-burden hour for an administrator at $\$ 107$ per hour $\times$ 1,353 CAHs).

22. ICRs Regarding CAH Provision of Services (§ 485.635(a)(4))

Section 485.635(a)(4) requires CAHs to conduct an annual review of all its policies and procedures (OMB control number 0938-1043). We are finalizing our proposal for revision of the patient care policies requirements imposed on
CAHs would reduce the frequency that is currently required for CAHs to perform a review of all their policies and procedures. We anticipate that a change from an annual review to a biennial review would reduce the burden on CAHs by half in a given period of time. For the purposes of our analysis, we estimate that it would take a CAH approximately 16 hours for administrative and clinical staff to review and make changes to policies and procedures annually. In a 2-year period, we estimate a savings of $\$ 1,968$ per facility, and a combined total savings of $\$ 2.7$ million for CAHs $(\$ 1,968$ $\times 1,353 \mathrm{CAHs}$ ), or annualized savings of approximately $\$ 1.3$ million.

We estimate that the CAH staff time and associated costs would be assigned to a biennial review as shown in Table 6.

TABLE 6-HOURLy COSTS AND BURDEN HOURS

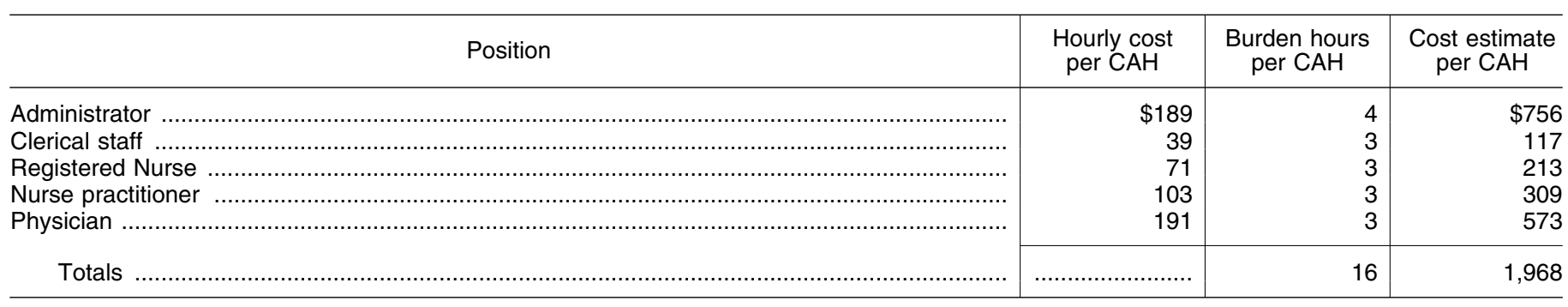

\section{ICRs Regarding Special} Requirements for CAH Providers of Long-Term Care Services ("SwingBeds”) (§ 485.645(d)(1), (4), (5) and (8))

We have included the discussion of the ICRs regarding special requirements for $\mathrm{CAH}$ providers of long-term care services in the discussion of the ICRs regarding special requirements for hospital providers of long-term care services, which can be found in section I.C.13 of this rule (ICRs Regarding Special Requirements for Hospital Providers of Long-Term Care Services ("Swing Beds") (§ 482.58(b)(1), (4), (5), and (8), and Parallel CAH requirements: $\S 485.645(\mathrm{~d})(1),(4),(5)$, and (8))).

\section{ICRs Regarding CMHCs} (§ 485.914(d))

Section 485.914(d)(1) requires each CMHC to update each client's comprehensive assessment via the CMHC interdisciplinary treatment team, in consultation with the client's primary health care provider (if any), no less frequently than every 30 days. We are modifying the requirement at $\S 485.914(\mathrm{~d})$ to remove the 30-day assessment update timeframe for those clients who do not receive PHP services. Under the current 30-day time frame requirement, each client receives an updated assessment 12 times per year (OMB control number 0938-1245). We estimate that, in accordance with the proposed need-based assessment update requirements, each non-PHP client would receive 2 assessment updates in a year. Therefore, we estimate that this change would reduce the burden of 10 assessments per client, per year.

As of August 2017 there are 161 Medicare participating CMHCs serving 3,122 Medicare beneficiaries and an estimated 2,080 non-Medicare clients, for an average of 32 clients per CMHC. In order to develop the estimated number of non-Medicare clients we divided the total number of Medicare beneficiaries who received partial hospitalization services by the total number of Medicare-participating CMHCs to establish the average number of Medicare beneficiaries per CMHC. This resulted in 19 beneficiaries per CMHC. We then assumed that, in order to comply with the 40 percent requirement ( $\$ 485.918(\mathrm{~b})(1)(\mathrm{v})$ ), those 19 beneficiaries only accounted for 60 percent of an average CMHC's total patient population. This means that an average CMHC also treated another 13 clients who did not have Medicare as a payer source, for a total of 32 clients (Medicare + non-Medicare) in an average CMHC. Therefore, all CMHCs combined would have approximately 2,093 non-PHP clients per year (13 per CMHC), and approximately 20,930 assessments would be reduced nationwide per year $(2,093$ patients $\times 10$ assessments per patient). We estimate that documenting each assessment update requires 10 minutes of a CMHC clinician's time, for a total savings of 3,487 hours nationwide $(0.1666$ hours $\times$ 20,930 assessment updates). At a cost of $\$ 7.50$ for a mental health counselor to document each assessment, the total cost savings would be $\$ 156,975$ (\$7.50 $\times 20,930$ assessments).

25. ICRs Regarding Portable X-Ray Services (§§ 486.104(a) and 486.106(a))

We are finalizing our proposal to revise the requirements for portable $\mathrm{x}$ ray technologist personnel qualifications at $\$ 486.104$ to align the current requirements at $\S 486.104$ (a)(1), (2), (3), and (4) with those for hospital radiologic technologists at $\$ 482.26$ (c)(2) which are focused on the qualifications of the individual performing services as permitted by State law. Although changing the qualifications would 
require management time, with the associated cost of those hours, in order to revise the internal personnel descriptions and qualifications, we believe that this proposed change would impose no burden because maintaining internal personnel descriptions and qualifications is a standard business practice. Therefore, this burden would not be subject to the PRA in accordance with the implementing regulations of the PRA at 5 CFR 1320.3(b)(2).

We are finalizing our proposal to revise the requirements for portable $\mathrm{x}$ ray orders at $\S 486.106(\mathrm{a})(2)$. We proposed to remove the requirement that physician or non-physician practitioner's orders for portable x-ray services must be written and signed. We also proposed to replace the specific requirements related to the content of each portable x-ray order with a crossreference to the requirements at $42 \mathrm{CFR}$ 410.32, which also apply to portable xray services. These changes would simplify the ordering process for portable x-rays and promote the use of more efficient ordering methods, such as electronic orders.

In the information collection request (OMB control number 0938-0338) we estimate that the current order requirements would impose the following burdens:

- 3 minutes to write an order $x$ $3,986,000$ portable $\mathrm{x}$-rays exams ordered
$=199,300$ hours $\times \$ 71 /$ hour for a nurse $=\$ 14,150,300$.

- $\$ 1$ for printing and faxing verbal orders to physician offices for signature $\times 2,500,000$ verbal orders $=\$ 2,500,000$.

- 2,000,000 follow-up calls regarding the status of faxes $\times 10$ minutes of time for clerical staff ( 5 minutes for portable x-ray clerical staff +5 minutes for ordering physician clerical staff) $=$ 333,333 hours $\times \$ 33 /$ hour $=$ $\$ 10,999,989$.

All of these burdens would be eliminated by revising the current ordering standards. Therefore, we estimate a proposed information collection savings of $\$ 27.7$ million from this proposed change.

26. ICRs Regarding RHC and FQHC Provision of Services ( $(491.9(\mathrm{~b})(4)$ )

There are currently more than 4,100 RHCs and approximately 1,400 FQHC organizations furnishing services at approximately 12,000 or more total locations. Many FQHC organizations have multiple delivery sites, and as of May 2017 there were 4,160 RHC and 7,874 FQHC delivery sites. All CMScertified sites are subject to our requirements and we are therefore utilizing the total number of current sites in our burden reduction calculations.

We are finalizing our proposal to revise $\S 491.9(b)(4)$ to reduce the number of times that RHCs and FQHCs perform a review of all their policies and procedures. Changing from an annual review to a review every other year would reduce the burden on RHCs and FQHCs by half in a given period of time. In the currently approved information collection request (OMB control number 0938-0334), we only included burden estimates for RHCs. However, we recognize that the information collection applies to FQHCs as well. Therefore, we estimate that it would take a RHC or FQHC approximately 4 hours for clinical staff to review and make changes to policies and procedures annually, for a total of 48,136 hours for all 12,034 RHC and FQHC locations. In a 2-year period, RHCs and FQHCs would use 96,272 total hours to comply with the requirements to annually review all of their policies and procedures. Under the proposed change to review policies every other year, we estimate that in a 2-year period, it will take a total of 48,136 hours, for a savings of 48,136 hours per year. We estimate a savings of $\$ 608$ per facility (see Table 7) for a combined total savings of $\$ 7.3$ million biennially for 12,034 RHCs or FQHCs $(\$ 608 \times 12,034$ RHCs and FQHCs $)$, or annualized savings of half these amounts. We will submit a revised information collection request to $\mathrm{OMB}$ for approval.

TABLE 7-HOURLy WAGES AND BuRDEN HOURS

\begin{tabular}{|c|c|c|c|}
\hline Position & $\begin{array}{l}\text { Hourly cost per } \\
\text { RHC/FQHC } \\
\text { (includes } 100 \% \\
\text { for benefits } \\
\text { and overhead) }\end{array}$ & $\begin{array}{l}\text { Burden hours per } \\
\text { RHC/FQHC }\end{array}$ & $\begin{array}{l}\text { Cost estimate per } \\
\text { RHC/FQHC }\end{array}$ \\
\hline Physician Mid-Level Provider (PA or NP) & $\begin{array}{r}\$ 203 \\
101\end{array}$ & $\begin{array}{l}2 \\
2\end{array}$ & $\begin{array}{r}\$ 406 \\
202\end{array}$ \\
\hline 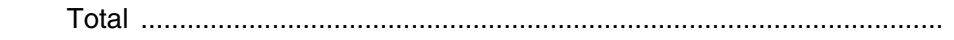 & (n) & 4 & 608 \\
\hline
\end{tabular}

27. ICRs Regarding RHC and FQHC Program Evaluation (§ 491.11(a))

We are finalizing the proposal to revise $\S 491.11$ (a) to reduce the number of times that RHCs and FQHCs carry out or arrange for an annual evaluation of the total program. Changing from an annual evaluation to an evaluation every other year would reduce the burden on RHCs and FQHCs by half in a given period of time. In the currently approved information collection request
(OMB control number 0938-0334), we only included burden estimates for RHCs, however we recognize that the information collection applies to FQHCs as well. Therefore, we estimate that it would take a RHC or FQHC approximately 6 hours for administrative and clinical staff to perform an evaluation of its total program annually for a total of 72,204 hours for all 12,034 RHC and FQHC locations. In a 2-year period, RHCs and FQHCs would use 144,408 total hours to comply with the requirement for an evaluation of the total program. Under the proposed change to evaluate the total program every other year, we estimate an hourly savings of 72,204 total hours and a cost savings of $\$ 822$ per facility (see Table 8), for a combined total savings of $\$ 9.9$ million biennially for 12,034 RHCs or FQHCs (\$822 $\times$ 12,034 RHC and FQHC locations), or annualized savings of half these amounts. 
TABLE 8-HOURLY WAGES AND BURDEN HOURS

\begin{tabular}{|c|c|c|c|}
\hline Position & $\begin{array}{l}\text { Hourly cost per } \\
\text { RHC/FQHC } \\
\text { (includes } 100 \% \text { for } \\
\text { benefits } \\
\text { and overhead) }\end{array}$ & $\begin{array}{l}\text { Burden hours per } \\
\text { RHC/FQHC }\end{array}$ & $\begin{array}{l}\text { Cost estimate per } \\
\text { RHC/FQHC }\end{array}$ \\
\hline $\begin{array}{l}\text { Administrator/Health Services Manager } \\
\text { Physician } \\
\text { Mid-Level Provider (PA or NP) }\end{array}$ & $\begin{array}{r}\$ 107 \\
203 \\
101\end{array}$ & $\begin{array}{l}2 \\
2 \\
2\end{array}$ & $\begin{array}{r}\$ 214 \\
406 \\
202\end{array}$ \\
\hline Total & 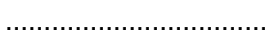 & 6 & 822 \\
\hline
\end{tabular}

28. ICRs Regarding Emergency Preparedness for Providers and Suppliers

a. Review of the Emergency Preparedness Program

At $\S 482.15(\mathrm{a}),(\mathrm{b}),(\mathrm{c})$, and (d) for hospitals and parallel regulatory citations for other facilities, we are finalizing our proposal to allow providers to review their program at least every 2 years. However, we are withdrawing the proposal for LTC facilities. As of May 2017, there were approximately 72,646 total facilities, or 56,983 excluding LTC facilities. All are required to review their emergency preparedness program annually, which includes a review of their emergency plan, policies and procedures, communication plan, and training and testing program.

For our analysis, we estimate that reducing this requirement from annually to biennially would reduce compliance costs related to review of the emergency plan by 50 percent. The methodology used for our cost estimate analysis generally mirrors the methodology used for the annual review of the emergency plan Emergency Preparedness final rule (81 FR 63930) with a 50 percent reduction in the cost estimate calculation; however, after receiving additional feedback from stakeholders, we have determined that we underestimated the amount of time it would take to review the emergency plan. As a result, we have presented current burden hours associated with reviewing the emergency plan that reflects the increased associated burden hours relative to the information collection request for this provision (OMB control number 0938-1325). As in the Emergency Preparedness final rule (81 FR 63930), we assume that the individuals involved in the review of the emergency plan include an administrator, director of nursing, a RN, a physician, a social worker, a counselor, and an office manager, depending on the facility type. Based on May 2017 BLS salary data, we calculated the hourly mean wage for each position for this requirement identified in the Emergency Preparedness final rule (81 FR 63930).

We estimate that the finalized change will accrue total annualized cost savings of $\$ 69,639,324$ and 657,345 burden hours saved, or biennial savings of double these amounts. We list a detailed calculation for each facility below, based on facility numbers available as of 2017:

- RNHCIs: Combined total savings of $\$ 9,684$ for 18 RNHCIs ( 8 burden hours for an administrator at $\$ 107$ plus 5 burden hours for a director of nursing at $\$ 44$ per hour) $\times 18$ RNHCIs $\times 50$ percent).

- ASCs: Combined total savings of $\$ 6,257,182$ for 5,557 ASCs ((8 burden hours for an administrator at \$109 per hour plus 4 burden hours for a physician at \$203 per hour plus 8 burden hours for a quality improvement $\mathrm{RN}$ at $\$ 71$ per hour) $\times 5,557$ ASCs $\times 50$ percent).

- Hospices: Combined total savings of $\$ 5,916,502$ for 4,489 hospice facilities ( $(8$ burden hours for an administrator at an hourly wage of \$107 per hour plus 4 burden hours for a physician at $\$ 203$ per hour plus 4 burden hours for a counselor at $\$ 45$ per hour plus 4 burden hours for a social worker at $\$ 55$ per hour plus 8 burden hours for a $\mathrm{RN}$ at $\$ 71$ per hour) $\times 4,489$ hospices $\times 50$ percent).

- PRTFs: Combined total savings of \$569,976 for 374 PRTFs ( 8 burden hours for an administrator $\$ 107$ per our plus 8 burden hours for a physician at $\$ 203$ per hour plus 8 burden hours for a $\mathrm{RN}$ at $\$ 71$ per hour) $\times 374 \mathrm{PRTFs} \times 50$ percent).

- PACE: Combined total savings of $\$ 232,068$ for 233 PACE organizations ((8 burden hours for an administrator at $\$ 107$ per hour plus 8 burden hours for a home care coordinator at $\$ 71$ per hour plus 8 burden hours for a RN at $\$ 71$ per hour) $\times 233$ PACE organizations $\times 50$ percent).

- Hospitals: Combined total savings of $\$ 11,700,598$ for 4,823 hospitals ( $(8$ burden hours for an administrator at $\$ 109$ per hour plus 8 burden hours for a physician at $\$ 203$ per hour plus 8 burden hours for a risk management director at $\$ 107$ per hour plus 8 burden hours for a quality assurance nurse at $\$ 71$ per hour plus 8 burden hours for a facility director at $\$ 99$ per hour plus 4 burden hours for a medical secretary at $\$ 35$ per hour $\times 4,823$ hospitals $\times 50$ percent).

- ICF/IID: Combined total savings $\$ 3,475,290$ for 6,097 ICF/IIDs ( 8 burden hours for an administrator at $\$ 107$ per hour plus 4 burden hours for a RN $\$ 71$ per hour) $\times 6,097$ ICF/IIDs $\times 50$ percent).

- $H H A$ : Combined total savings of $\$ 16,512,192$ for 12,624 HHAs ( 8 burden hours for an administrator at $\$ 107$ per hour plus 8 burden hours for a nursing director at $\$ 107$ per hour plus 8 burden hours for a director of rehab at $\$ 85$ per hour plus 4 burden hours for an office manager at $\$ 56$ per hour) $\times 12,624$ HHAs $\times 50$ percent)

- CORF: Combined total savings of $\$ 144,384$ for 188 CORFs ( 8 burden hours for an administrator at \$107 per hour plus 8 burden hours for a physical therapist at $\$ 85$ per hour) $\times 188$ CORFs $\times 50$ percent).

- CAH: Combined total savings of $\$ 1,693,956$ for 1,353 CAHs ( 8 burden hours for an administrator at $\$ 107$ per hour plus 8 burden hours for a director of nursing at $\$ 107$ per hour plus 8 burden hours for a facility director at $\$ 99$ per hour) $\times 1,353$ CAHs $\times 50$ percent).

- Organizations: Combined total savings of $\$ 1,241,448$ for 2,076 Organizations ( $(8$ burden hours for an administrator at \$107 per hour plus 4 burden hours for a physical therapist at $\$ 85$ per hour) $\times 2,076$ Organizations $\times$ 50 percent).

- CMHCs: Combined total savings of $\$ 150,052$ for 161 CMHCs ( 8 burden hours for an administrator at \$107 per hour plus 8 burden hours for a $\mathrm{RN}$ at $\$ 71$ per hour plus 8 burden hours for a social worker at $\$ 55$ per hour) $\times 161$ CMHCs $\times 50$ percent).

- OPOs: Combined total savings of $\$ 121,568$ for 58 OPOs ( 8 burden hours for an OPO director at \$107 per hour plus 8 burden hours for a physician at $\$ 203$ per hour plus 8 burden hours for 
a QAPI director at $\$ 107$ per hour plus 8 burden hours for an organ procurement coordinator at $\$ 107$ per hour) $\times 58$ OPOs $\times 50$ percent).

- $R H C / F Q H C$ : Combined total savings of $\$ 10,108,560$ ( $(8$ burden hours for an administrator at $\$ 107$ per hour plus 8 burden hours for a nurse practitioner/ physician assistant at $\$ 103$ per hour) $\times$
4,160 RHCs $\times 50$ percent) $\$ 3,494,400+$ ( 8 burden hours for an administrator at $\$ 107$ per hour plus 8 burden hours for a nurse practitioner/physician assistant at $\$ 103$ per hour $\times 7,874$ FQHCs $\times 50$ percent) $\$ 6,614,160)$.

- ESRD Facilities: Combined total savings of $\$ 11,505,864$ for 6,898 dialysis facilities (( 8 burden hours for an administrator at $\$ 107$ per hour plus 8 burden hour for a medical director/ physician at \$203 per hour plus 8 burden hours for a nurse manager at $\$ 107) \times 6,898$ dialysis facilities $\times 50$ percent) as shown in Table 9.

Table 9-Cost Savings for Annual Review of Emergency Preparedness Plan

\begin{tabular}{|c|c|c|}
\hline Provider/supplier & $\begin{array}{l}\text { Cost savings per } \\
\text { provider/supplier }\end{array}$ & Combined total savings \\
\hline RNHCls ... & $\$ 538$ & $\$ 9,684$ for $18 \mathrm{RNHCls.}$ \\
\hline 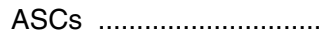 & 1,126 & $\$ 6,257,182$ for 5,557 ASCs. \\
\hline Hospices ..................... & 1,318 & $\$ 5,916,502$ for 4,489 hospice facilities both inpatient and freestanding/home based. \\
\hline PRTFs $\ldots \ldots \ldots \ldots \ldots \ldots \ldots \ldots$ & 1,524 & $\$ 569,976$ for 374 PRTFs. \\
\hline PACEs ................ & 996 & $\$ 232,068$ for 233 PACEs. \\
\hline Hospitals ...... & 2,426 & $\$ 11,700,598$ for 4,823 hospitals. \\
\hline ICFs/IIDs $\ldots \ldots \ldots \ldots \ldots \ldots \ldots$ & 570 & $\$ 3,475,290$ for 6,097 ICF/IIDs. \\
\hline HHAs ................ & 1,308 & $\$ 16,512,192$ for 12,624 HHAs. \\
\hline 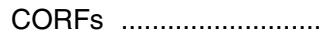 & 768 & $\$ 144,384$ for 188 CORFs. \\
\hline 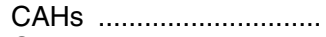 & 1,252 & $\$ 1,693,956$ for $1,353 \mathrm{CAHs}$. \\
\hline Organizations $\ldots \ldots \ldots \ldots \ldots$ & 598 & $\$ 1,241,448$ for 2,076 Organizations. \\
\hline $\mathrm{CMHCs} \ldots \ldots \ldots \ldots \ldots \ldots \ldots$ & 932 & $\$ 150,052$ for $161 \mathrm{CMHCs}$. \\
\hline OPOs $\ldots \ldots \ldots \ldots \ldots \ldots \ldots \ldots$ & 2,096 & $\$ 121,568$ for 58 OPOs. \\
\hline RHCs/FQHCs ................ & 840 & $\begin{array}{l}\$ 10,108,560 \text { for RHCs and FQHCs }(\$ 3,494,400 \text { for } 4,160 \text { RHCs and } \$ 6,614,160 \text { for } 7,874 \\
\text { FQHCs). }\end{array}$ \\
\hline ESRD Facilities & 1,668 & $\$ 11,505,864$ for 6,898 dialysis facilities. \\
\hline
\end{tabular}

b. Contents of the Emergency Plan

At § 482.15(a)(4) for hospitals, and other parallel citations for the facilities mentioned in section II.J.2 of the rule, we are finalizing our proposal to eliminate the requirement that facilities document efforts to contact local, tribal, regional, State, and Federal emergency preparedness officials and that facilities document participation in collaborative and cooperative planning efforts (OMB control number 0938-1325). We estimate that an administrator, or in the case of a hospital a community relations manager, a program director for a PACE, or a QAPI director for OPOs, would take 1 hour to document efforts to contact local, tribal, regional, State and Federal emergency preparedness officials and, when applicable, document the facility's participation in collaborative and cooperative planning efforts. We note that The Joint Commission (TJC)- accredited ASCs, TJC-accredited CAHs, and TJC-accredited hospitals have emergency preparedness requirements for developing an emergency preparedness plan that are comparable to the current emergency preparedness CoPs (81 FR 63937, 63954, and 63978 through 63979). Utilizing the same assumptions we used in the Emergency Preparedness final rule (81 FR 63937, 63954, and 63978 through 63979), we estimate that cost savings will accumulate from non-TJC accredited ASC, CAHs, and hospitals, since TJCaccredited ASCs, CAHs and hospitals are already required by the TJC to develop emergency preparedness plans. As a result, these facilities are excluded from the analysis given the requirements of their accreditation organization standards. Based on May 2017 BLS salary data, we calculate an hourly mean wage of $\$ 107$ for an administrator, a PACE Program Director, or QAPI director and a cost savings of \$107 per facility for RNHCIs, non-TJC accredited ASCs, hospices (both inpatient and freestanding), PRTFs, PACEs, LTCFs, ICF/IIDs, HHAs, CORFs, non-TJC accredited CAHs,

Organizations, CMHCs, OPOs, RHC/

FQHCs, and dialysis facilities (\$107 hourly mean wage $\times 1$ burden hour). For non-TJC accredited hospitals, we estimate an hourly mean wage of $\$ 115$ for a community relations manager, and a $\$ 115$ cost per facility $(\$ 115 \times 1$ hour $)$. Therefore, we estimate the following for each facility affected by the proposed change, for a total savings of $\$ 7,316,489$ and 68,275 burden hours. We list a summary of the calculation for savings accrued by removing this requirement for each facility in Table 10, based on facility numbers available as of May 2017.

\section{Table 10-Cost Savings: Documentation of the Facility's Participation in Collaborative and Cooperative} PLANNING EFFORTS

\begin{tabular}{|c|c|c|}
\hline Provider/supplier & $\begin{array}{l}\text { Cost savings per } \\
\text { provider/supplier }\end{array}$ & Combined total savings \\
\hline RNHCls ....................... & $\$ 107$ & $\$ 1,926$ for $18 \mathrm{RNHCls.}$ \\
\hline Hospices ........................ & 107 & $\$ 480,323$ for 4,489 hospice facilities both inpatient and freestanding/home based. \\
\hline PRTFs …..................... & 107 & $\$ 40,018$ for 374 PRTFs. \\
\hline PACEs …...................... & 107 & $\$ 24,931$ for 233 PACEs. \\
\hline
\end{tabular}


TABle 10-Cost SAVINGs: Documentation OF the FACILITY'S PARTICIPATION IN COLLABORATIVE AND CoOperative PLANNING EFFORTS-Continued

\begin{tabular}{|c|c|c|}
\hline Provider/supplier & $\begin{array}{l}\text { Cost savings per } \\
\text { provider/supplier }\end{array}$ & Combined total savings \\
\hline LTCFs & 107 & $\$ 1,675,941$ for 15,663 LTCFs. \\
\hline 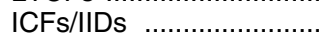 & 107 & $\$ 652,379$ for 6,097 ICF/IIDs. \\
\hline CORFs & 107 & $\$ 20,116$ for 188 CORFs. \\
\hline $\begin{array}{l}\text { CAHs (Non-TJC accred- } \\
\text { ited). }\end{array}$ & 107 & $\$ 107,428$ for 1,004 non-TJC accredited CAHs. \\
\hline $\mathrm{RHCs} / \mathrm{FQHCs} \ldots \ldots \ldots \ldots \ldots . . . .$. & 107 & $\$ 1,287,638$ for RHCs and FQHCs ( $\$ 445,120$ for 4,160 RHCs and $\$ 842,518$ for 7,874 FQHCs). \\
\hline ESRD Facilities & 107 & $\$ 738,086$ for 6,898 dialysis facilities. \\
\hline
\end{tabular}

\section{c. Training}

At $§ 482.15(d)(1)(i i)$ for hospitals, and other parallel citations for other facilities mentioned in section II.J.2 of the rule, we are finalizing our proposal to require that facilities provide training biennially, or every 2 years, after facilities conduct initial training on their emergency program, as well as requiring additional training when the emergency plan is significantly updated. However, we are withdrawing this proposal for LTC facilities only. We are maintaining the requirement that providers and suppliers develop a wellorganized, effective training program that includes initial training for new and existing staff in emergency preparedness policies and procedures and would require training when the emergency plan is significantly updated. Facilities will have the flexibility to determine what is considered a significant update to the emergency plan.

For our analysis, we estimate that reducing this requirement from annually to biennially will reduce compliance costs related to providing emergency preparedness training by 50 percent (OMB control number 09381325). The methodology used for our cost estimate analysis mirrors the methodology used for the annual training requirement in the Emergency Preparedness final rule (81 FR 63930) with a 50 percent reduction in the cost estimate calculation. As in the Emergency Preparedness final rule (81 FR 63930), we assume that the individuals involved in the development and provision of training include an administrator, director of nursing, a RN, and an office manager, depending on the facility type. Providers and suppliers are expected to provide initial training in emergency preparedness policies and procedures to all new and existing staff, individuals providing services under arrangement, and volunteers, consistent with their expected roles, and maintain documentation of the training. Based on May 2017 BLS salary data, we calculated the hourly mean wage for each position for this requirement identified in the Emergency Preparedness final rule (81 FR 63930). We estimate that the proposed change will accrue annualized cost savings of $\$ 25,593,781$ and 288,266 burden hours, or biennial savings of double these amounts. We list a detailed calculation for each facility below, based on facility numbers available as of May 2017 with a summary of these calculations provided in Table 11:

- RNHCIs: Combined total savings of $\$ 3,906$ for 18 RNHCIs ( 2 burden hours for an administrator at $\$ 107$ plus 5 burden hours for a director of nursing at $\$ 44$ per hour) $\times 18$ RNHCIs $\times 50$ percent).

- ASCs: Combined total savings of $\$ 1,289,224$ for 5,557 ASCs ((1 burden hour for an administrator at $\$ 109$ per hour plus 5 burden hours for a quality improvement $\mathrm{RN}$ at $\$ 71$ per hour) $\times$ 5,557 ASCs $\times 50$ percent).

- Hospices: Combined total savings of $\$ 956,157$ for 4,489 hospice facilities (6 burden hours for a $\mathrm{RN}$ at $\$ 71$ per hour $\times 4,489$ hospices $\times 50$ percent).

- PRTFs: Combined total savings of $\$ 132,770$ for 374 PRTFs (10 burden hours for a $\mathrm{RN}$ at $\$ 71$ per hour $\times 374$ PRTFs $\times 50$ percent).

- PACE: Combined total savings of $\$ 99,258$ for 233 PACE organizations (3 burden hours for a home care coordinator at $\$ 71$ per hour plus 9 burden hours for a $\mathrm{RN}$ at $\$ 71$ per hour $\times 233$ PACE organizations $\times 50$ percent)

- Hospitals: As we stated in the Emergency Preparedness final rule (81 FR 63958), TJC-accredited hospitals are required to train their staff for their assigned roles during emergencies (CAMH, Standard EC.4.16, Eps 1-2, p. EC-13e). In addition, the TJC-accredited hospitals also must provide on-going training to their staff, including training on specific job-related safety (CAMH, Standard HR-2.30, EP 4, CAMH Refreshed Core, January 2008, p. HR11), and we expect that emergency preparedness is part of such on-going training. As a result, we estimate a combined total savings of $\$ 2,066,202$ for 1,383 non-TJC accredited hospitals (2 burden hours for an administrator at $\$ 109$ per hour plus 6 burden hours for a risk management director at $\$ 107$ per hour plus 28 hours for a healthcare trainer (RN) at $\$ 71$ per hour plus 4 burden hours for a medical secretary at $\$ 35$ per hour $\times 1,383$ hospitals $\times 50$ percent).

- ICF/IID: Combined total savings $\$ 1,734,597$ for 6,097 ICF/IIDs (2 burden hours for an administrator at $\$ 107$ per hour plus 5 burden hours for a RN $\$ 71$ per hour $\times 6,097$ ICF/IIDs $\times 50$ percent).

- HHA: Combined total savings of $\$ 8,066,736$ for 12,624 HHAs (2 burden hours for an administrator at $\$ 107$ per hour plus 2 burden hours for a nursing director at $\$ 107$ per hour plus 2 burden hours for a director of rehab at $\$ 85$ per hour plus 2 burden hours for an office manager at $\$ 56$ per hour plus 8 burden hours for a director of training at $\$ 71 \times$ 12,624 HHAs $\times 50$ percent).

- CORF: Combined total savings of $\$ 74,260$ for 188 CORFs (5 burden hours for an administrator at $\$ 107$ per hour plus 3 burden hours for a physical therapist at $\$ 85$ per hour $\times 188 \mathrm{CORFs}$ $\times 50$ percent).

- CAH: Combined total savings of $\$ 997,161$ for 1,353 CAHs (2 burden hours for an administrator at \$107 per hour plus 9 burden hours for a director of nursing at $\$ 107$ per hour plus 3 burden hours for a facility director at $\$ 99$ per hour $\times 1,353 \mathrm{CAHs} \times 50$ percent).

- Organizations: Combined total savings of $\$ 842,856$ for 2,076 Organizations ( 6 burden hours for an administrator at $\$ 107$ per hour plus 2 burden hours for a physical therapist at 
$\$ 85$ per hour $\times 2,076$ Organizations $\times 50$ percent).

- CMHCs: Combined total savings of $\$ 57,155$ for 161 CMHCs (10 burden hours for a psychiatric RN at $\$ 71$ per hour $\times 161$ CMHCs $\times 50$ percent).

- OPOs: Combined total savings of $\$ 113,448$ for 58 OPOs (2 burden hours for a director at $\$ 115$ per hour plus 2 burden hours for a medical director/ physician at $\$ 203$ per hour plus 12 burden hours for a QAPI director at $\$ 107$ per hour plus 8 hours for an organ procurement coordinator at \$107 per hour plus 16 burden hours for an education coordinator at $\$ 71$ per hour $\times$ 58 OPOs $\times 50$ percent).

- $R H C / F Q H C$ : Combined total savings of $\$ 6,245,646$ ( $(2$ burden hours for an administrator at $\$ 107$ per hour plus 8 burden hours for a nurse practitioner/ physician assistant at $\$ 103$ per hour $\times$ 4,160 RHCs $\times 50$ percent) $\$ 2,159,040+$ ( 2 burden hours for an administrator at $\$ 107$ per hour plus 8 burden hours for a nurse practitioner/physician assistant at $\$ 103$ per hour $\times 7,874$ FQHCs $\times 50$ percent) $\$ 4,086,606)$.

- ESRD Facilities: Combined total savings of $\$ 2,914,405$ for 6,898 dialysis facilities (3 burden hours for an administrator at $\$ 107$ per hour plus 1 burden hour for a medical director/ physician at $\$ 203$ per hour plus 3 burden hours for a nurse manager at $\$ 107 \times 6,898$ dialysis facilities $\times 50$ percent).

TABLE 11-Cost SAVINGS: TRAINING

\begin{tabular}{|c|c|c|}
\hline $\begin{array}{c}\text { Provider/ } \\
\text { supplier }\end{array}$ & $\begin{array}{c}\text { Cost savings per } \\
\text { provider/ } \\
\text { supplier }\end{array}$ & Combined total savings \\
\hline RNHCls .... & $\$ 217$ & $\$ 3,906$ for $18 \mathrm{RNHCls.}$ \\
\hline 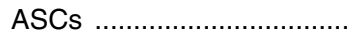 & 232 & $\$ 1,289,224$ for 5,557 ASCs. \\
\hline Hospices ........................... & 213 & $\$ 956,157$ for 4,489 hospice facilities both inpatient and freestanding/home based. \\
\hline PRTFs ............................. & 355 & $\$ 132,770$ for 374 PRTFs. \\
\hline PACEs $\ldots . . .$. & 426 & $\$ 99,258$ for 233 PACE organizations. \\
\hline $\begin{array}{l}\text { Hospitals (Non-TJC ac- } \\
\text { credited). }\end{array}$ & 1,494 & $\$ 2,066,202$ for 1,383 non-TJC accredited hospitals. \\
\hline ICFs/IIDs ........ & 285 & $\$ 1,734,597$ for 6,097 ICF/IIDs. \\
\hline HHAs $\ldots \ldots \ldots \ldots \ldots \ldots \ldots \ldots \ldots$ & 639 & $\$ 8,066,736$ for 12,624 HHAs. \\
\hline CORFs $\ldots \ldots \ldots \ldots \ldots \ldots \ldots \ldots \ldots \ldots \ldots \ldots \ldots \ldots \ldots \ldots$ & 395 & $\$ 74,260$ for 188 CORFs. \\
\hline 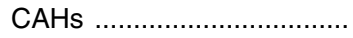 & 737 & $\$ 997,161$ for 1,353 CAHs. \\
\hline Organizations ...................... & 406 & $\$ 842,856$ for 2,076 Organizations. \\
\hline CMHCs $\ldots \ldots \ldots \ldots \ldots \ldots \ldots \ldots$ & 355 & $\$ 57,155$ for $161 \mathrm{CMHCs}$. \\
\hline 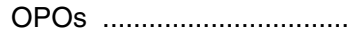 & 1,956 & $\$ 113,448$ for 58 OPOs. \\
\hline RHCs/FQHCs ..................... & 519 & $\begin{array}{l}\$ 6,245,646 \text { for RHCs and FQHCs }(\$ 2,159,040 \text { for } 4,160 \text { RHCs and } \$ 4,086,606 \text { for } 7,874 \\
\text { FQHCs). }\end{array}$ \\
\hline ESRD Facilities .................. & 423 & $\$ 2,914,405$ for 6,898 dialysis facilities. \\
\hline
\end{tabular}

\section{d. Testing}

Finally, at $\S 482.15(d)(2)$, we are finalizing our proposal to require that providers of inpatient services mentioned in section II.J.2 of the rule conduct two testing exercises annually, one of which may be an exercise of their choice that must be either a communitybased full-scale exercise (if available), an individual facility-based functional exercise, a drill, a tabletop exercise or workshop that includes a group discussion led by a facilitator. Given that these providers are currently required to conduct two testing exercises annually, and because they may choose to conduct the same types of testing exercises, we do not anticipate that this requirement will impose a burden upon providers of inpatient services and as such, this revision will not result in a savings of burden hours or dollars (OMB control number 09381325).

We are also finalizing our proposal to require that providers of outpatient services mentioned in section II.J.2 of the rule conduct one testing exercise annually which must be either a community-based full-scale exercise (if available) or an individual facility-based functional exercise every other year, and in the opposite years, may be either a community-based full-scale exercise (if available), a facility-based functional exercise, a drill, or a tabletop exercise or workshop that includes a group discussion led by a facilitator.

For our analysis, we estimate that reducing this requirement from biannually to annually for outpatient providers will reduce compliance costs related to conducting emergency preparedness testing by 50 percent. The methodology used for our cost estimate analysis mirrors the methodology used for the biannual testing requirement in the Emergency Preparedness final rule (81 FR 63930) with a 50 percent reduction in the cost estimate calculation. As in the Emergency Preparedness final rule (81 FR 63930), we will assume that the same individuals involved with developing training would typically also develop the scenarios, materials, as well as any accompanying documentation associated with testing exercises. Based on May 2017 BLS salary data, we calculated the hourly mean wage for each position for this requirement identified in the Emergency Preparedness final rule (81 FR 63930) and decreased the cost by 50 percent due to the 50 percent reduction in the frequency requirement.

We estimate that the proposed change will accrue a total annual cost savings of $\$ 9,296,423$ and 100,969 burden hours. We list a detailed calculation for each facility below, based on facility numbers available as of May 2017 with a summary of these calculations provided in Table 12:

- ASCs: Combined total savings of $\$ 1,091,951$ for 5,557 ASCs ((1 burden hour for an administrator at $\$ 109$ per hour plus 4 burden hours for a quality improvement RN at $\$ 71$ per hour) $\times$ 5,557 ASCs $\times 50$ percent).

- Freestanding/home-based hospices: Combined total savings of $\$ 573,680$ for 4,040 hospice facilities (4 burden hours for a $\mathrm{RN}$ at $\$ 71$ per hour $\times 4,040$ hospices $\times 50$ percent).

- PACE: Combined total savings of $\$ 41,358$ for 233 PACE organizations (4 burden hours for a home care coordinator at $\$ 71$ per hour plus 1 burden hours for a $\mathrm{RN}$ at $\$ 71$ per hour $\times 233$ PACE organizations $\times 50$ percent).

- HHA: Combined total savings of $\$ 4,039,680$ for 12,624 HHAs (1 burden hour for an administrator at $\$ 107$ per hour plus 3 burden hours for a nursing 
director at $\$ 107$ per hour plus 1 burden hours for a director of rehab at $\$ 85$ per hour plus 1 burden hour for an office manager at \$56 per hour plus 1 burden hours for a director of training at $\$ 71 \times$ 12,624 HHAs $\times 50$ percent).

- CORF: Combined total savings of $\$ 56,212$ for 188 CORFs (4 burden hours for an administrator at $\$ 107$ per hour plus 2 burden hours for a physical therapist at $\$ 85$ per hour $\times 188$ CORFs $\times 50$ percent)

- Organizations: Combined total savings of $\$ 310,362$ for 2,076 organizations (2 burden hours for an administrator at $\$ 107$ per hour plus 1 burden hour for a physical therapist at
$\$ 85$ per hour $\times 2,076$ organizations $\times 50$ percent).

- CMHCs: Combined total savings of $\$ 22,862$ for 161 CMHCs (4 burden hours for a psychiatric $\mathrm{RN}$ at $\$ 71$ per hour $\times$ 161 CMHCs $\times 50$ percent).

- OPOs: Combined total savings of $\$ 13,427$ for 58 OPOs (3 burden hours for a QAPI director at $\$ 107$ per hour plus 2 burden hours for an education coordinator at $\$ 71$ per hour $\times 58$ OPOs $\times 50$ percent).

- RHC/FQHC: Combined total savings of $\$ 3,146,891$ ( $(2$ burden hours for an administrator at $\$ 107$ per hour plus 3 burden hours for a nurse practitioner/ physician assistant at $\$ 103$ per hour $\times$
4,160 RHCs $\times 50$ percent) $\$ 1,087,840+$ ( 2 burden hours for an administrator at $\$ 107$ per hour plus 3 burden hours for a nurse practitioner/physician assistant at $\$ 103$ per hour $\times 7,874$ FQHCs $\times 50$ percent) $\$ 2,059,051)$.

- ESRD: As identified in the Emergency Preparedness final rule (81 FR 64006), the current CFCs already require dialysis facilities to evaluate their emergency preparedness plan at least annually (§ 494.60(d)(4)(ii)); thus, we expect that all dialysis facilities are already conducting some type of tests to evaluate their emergency preparedness plans. As a result, Dialysis facilities are not included in the burden calculation.

TABLE 12-COSt SAVINGS: TESTING

\begin{tabular}{|c|c|c|}
\hline $\begin{array}{l}\text { Provider/ } \\
\text { supplier }\end{array}$ & $\begin{array}{l}\text { Cost savings per } \\
\text { provider/supplier }\end{array}$ & Combined total savings \\
\hline ASCs & $\$ 197$ & $\$ 1,091,951$ for 5,557 ASCs. \\
\hline $\begin{array}{l}\text { Hospices (freestanding/ } \\
\text { home-based). }\end{array}$ & 142 & $\$ 573,680$ for 4,040 hospices. \\
\hline 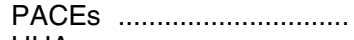 & 178 & $\$ 41,358$ for 233 PACE organizations. \\
\hline 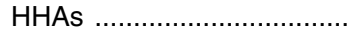 & 320 & $\$ 4,039,680$ for 12,624 HHAs. \\
\hline CORFs $\ldots \ldots \ldots \ldots \ldots \ldots \ldots \ldots \ldots$ & 299 & $\$ 56,212$ for 188 CORFs. \\
\hline OPOs $\ldots \ldots \ldots \ldots \ldots \ldots \ldots \ldots \ldots \ldots \ldots \ldots \ldots \ldots \ldots$ & 232 & $\$ 13,427$ for 58 OPOs. \\
\hline RHCs/FQHCs ...................... & 262 & $\$ 3,146,891$ ( $\$ 1,087,840$ for 4,160 RHCs and $\$ 2,059,051$ for 7,874 FQHCs). \\
\hline
\end{tabular}

We received few comments specifically addressing our information collection cost and burden estimates. Many comments, as previously discussed, did address specific regulatory changes and with only a few exceptions, mainly related to long term care facilities, endorsed those proposals to reduce information collection burdens.

We will submit a revised information collection request to OMB to account for the burden hour and cost savings.

II. Final Rule: Fire Safety Requirements for Certain Dialysis Facilities

\section{A. Background}

\section{Overview}

The Life Safety Code (LSC) is a compilation of fire safety requirements for new and existing buildings, and is updated and published every 3 years by the National Fire Protection Association (NFPA), a private, nonprofit organization dedicated to reducing loss of life due to fire. The Medicare and Medicaid regulations have historically incorporated these requirements by reference, along with Secretarial waiver authority. The statutory basis for incorporating NFPA's LSC into the regulations we apply to Medicare and, as applicable, Medicaid providers and suppliers is the Secretary of the
Department of Health and Human Services' (the Secretary) authority to stipulate health, safety and other regulations for each type of Medicare and (if applicable) Medicaidparticipating facility. Specifically, section 1881(b)(1)(A) of the Social Security Act (the Act) provides for payments for "providers of services and renal dialysis facilities which meet such requirements as the Secretary shall by regulation prescribe for institutional dialysis services and supplies. . . ." Under this statutory authority, the Secretary has set out "Conditions for Coverage," including LSC compliance requirements, at 42 CFR part 494 , subpart B. Our current LSC provisions are set out at $\S 494.60(\mathrm{~d})$.

In implementing the LSC provisions, we have given ourselves the discretion to waive specific provisions of the LSC for facilities if application of our rules would result in unreasonable hardship for the facility, and if the health and safety of its patients would not be compromised by such waiver. For dialysis facilities, that authority is set out at $\S 494.60(d)(4)$. In addition, the Secretary may accept a State's fire and safety code instead of the LSC if the Centers for Medicare \& Medicaid Services (CMS) determines that the protections of the State's fire and safety code are equivalent to, or more stringent than, the protections offered by the LSC; dialysis facility provisions to that effect are set out at $\S 494.60(\mathrm{~d})(3)$. These flexibilities mitigate the potential unnecessary burdens of applying the requirements of the LSC to all affected health care facilities.

On May 12, 2012, we published a final rule in the Federal Register, entitled "Medicare and Medicaid Program; Regulatory Provisions to Promote Program Efficiency, Transparency, and Burden Reduction" (77 FR 29002). In that final rule, we limited the application of LSC requirements to dialysis facilities either located adjacent to industrial high hazard areas, and those that did not provide one or more exits to the outside at grade level from the patient treatment area level. Subsequently, we proposed to update Life Safety Code provisions for CMS providers and suppliers, "Medicare and Medicaid Programs; Fire Safety Requirements for Certain Health Care Facilities; Proposed Rule"' (79 FR 21552, April 16, 2014). However, we inadvertently neglected to include dialysis facilities in this proposal. Therefore, we issued a proposal specifically for dialysis facilities, "Medicare and Medicaid Programs; Fire Safety Requirements for Certain Dialysis 
Facilities"' (81 FR 76899, November 4, 2016). We are finalizing these provisions now, with some modifications to the terms of the LSC to address the unique needs of dialysis facilities. The finalized update would apply only to dialysis facilities that do not provide one or more exits to the outside at grade level from the treatment area level (for instance, in upper floors of a mid-rise or high-rise building). We would not require other dialysis facilities to comply with NFPA $99^{\circledR}$ 2012 edition of the Health Care Facilities Code (NFPA 99) and NFPA $101^{\circledR} 2012$ edition of the Life Safety Code (NFPA 101) because we believe that patients in dialysis facilities are generally capable of unhooking themselves from dialysis machines and self-evacuating without additional assistance in the event of an emergency. We believe that in all facilities with atgrade exits, patients would be able to evacuate the building in a timely fashion. Consequently, we believe that state and local requirements are sufficient to protect these patients and staff in the event of an emergency. In accordance with NFPA 101 sections 20.1.3.7 and 21.1.3.7, we would prohibit Medicare-approved dialysis facilities from being located adjacent to industrial high hazard facilities. "Adjacent to" is defined as sharing a wall, ceiling or floor, with a facility.

Defining "Exit to the Outside at Grade Level From the Patient Treatment Area Level",

The phrase "exit to the outside at grade level from the patient treatment area level" applies to dialysis facilities that are on the ground or grade level of a building where patients do not have to traverse up or down stairways within the building to evacuate to the outside. Accessibility ramps in the exit area that provide an ease of access between the patient treatment level and the outside ground level are not considered stairways.

A dialysis facility which provides one or more exits to the outside at grade level from patient treatment level and which has a patient exit path to the outside (which may include an accessibility ramp that is compliant with NFPA and the Americans with Disabilities Act (ADA)) would be exempt from compliance with the applicable provisions of NFPA 99 and NFPA 101

\section{B. Provisions of the Proposed Rule and Analysis and Response to Public Comments}

On November 4, 2016 we published a proposed rule to update the requirements for certain dialysis facilities (81 FR 76899) that do not provide one or more exits to the outside at grade level from the patient treatment area to comply with the 2012 edition of the NFPA 101 and NFPA 99.

We are finalizing those requirements for dialysis facilities that do not provide one or more exits to the outside at grade level from the patient treatment area level, by incorporating a reference to the 2012 edition of NFPA 101 and NFPA 99. Certified dialysis facilities without one or more exits to the outside at grade level from the patient treatment area level are already required to meet the 2000 edition of the LSC, while other provider and supplier types are required to comply with the 2012 edition of the NFPA 101 and the NFPA 99 (LSC final rule published May 4, 2016 at 81 FR 26872).

The NFPA $101^{\circledR} 2012$ edition of the LSC provides minimum requirements, with due regard to function, for the design, operation and maintenance of buildings and structures for safety to life from fire. Its provisions also aid life safety in similar emergencies.

The NFPA $99^{\circledR} 2012$ edition of the Health Care Facilities Code provides minimum requirements for health care facilities for the installation, inspection, testing, maintenance, performance, and safe practices for facilities, material, equipment, and appliances.

\section{2012 Edition of the Life Safety Code}

The 2012 edition of the LSC includes new provisions that we believe are vital to the health and safety of all patients and staff. Our intention is to ensure that patients and staff continue to experience the highest degree of fire safety possible. We do review each edition of the NFPA 101 and NFPA 99 every 3 years to see if there are any significant provisions that we need to adopt. CMS will continue to review revisions to ensure we meet proper standards for patient safety. We have reviewed the 2015 and 2018 edition of the NFPA 101 and NFPA 99 and do not believe that there are any significant provisions that need to be addressed at this time. Newer buildings are typically built to comply with the newer versions of the LSC because state and local jurisdictions often adopt and enforce newer versions of the LSC as they become available.

We must emphasize that the LSC is not an accessibility code, and compliance with the LSC does not ensure compliance with the requirements of the ADA. State and local government programs and services, including health care facilities, are required to comply with Title II of the ADA. Private entities that operate public accommodations such as nursing homes, hospitals, and social service center establishments are required to comply with Title III of the ADA. Entities that receive federal financial assistance from the Department of Health and Human Services, including Medicare and Medicaid, are also required to comply with section 504 of the Rehabilitation Act of 1973. The same accessibility standards apply regardless of whether health care facilities are covered under Title II or Title III of the ADA or section 504 of the Rehabilitation Act of $1973 .{ }^{1}$ For more information about the ADA's requirements, see the Department of Justice's website at http://www.ada.gov or call 1-800-514-0301 (voice) or 1800-514-0383 (TTY).

\section{Incorporation by Reference}

This final rule will incorporate by reference the NFPA $101^{\circledR} 2012$ edition of the LSC, issued August 11, 2011, and Tentative Interim Amendments (TIAs) issued prior to April 16, 2014; and the NFPA $99^{\circledR} 2012$ edition of the Health Care Facilities Code, issued August 11, 2011, and TIAs issued prior to April 16, 2014 in $\S 494.60$ (f).

(1) NFPA 101, Life Safety Code, 2012

edition, issued August 11, 2011;

(i) TIA 12-1 to NFPA 101, issued

August 11, 2011.

(ii) TIA 12-2 to NFPA 101, issued

October 30, 2012.

(iii) TIA $12-3$ to NFPA 101, issued

October 22, 2013.

(iv) TIA 12-4 to NFPA 101, issued October 22, 2013.

(2) NFPA 99, Standards for Health Care Facilities Code of the National Fire Protection Association 99, 2012 edition, issued August 11, 2011.

(i) TIA $12-2$ to NFPA 99 , issued

August 11, 2011.

(ii) TIA $12-3$ to NFPA 99, issued August 9, 2012.

(iii) TIA $12-4$ to NFPA 99, issued March 7, 2013.

(iv) TIA $12-5$ to NFPA 99, issued

August 1, 2013.

(v) TIA $12-6$ to NFPA 99, issued March 3, 2014.

These materials have been previously incorporated by reference for other

\footnotetext{
${ }^{1}$ Facilities newly constructed or altered after March 15, 2012 must comply with the 2010 Standards for Accessible Design (2010 Standards). Facilities newly constructed or altered between September 15, 2010 and March 15, 2012 had the option of complying with either the 1991 Standards for Accessible Design (1991 Standards) or the 2010 Standards. Facilities newly constructed between January 26, 1993 and September 15, 2010, or altered between January 26, 1992 and September 15, 2010 were required to comply with the 1991 Standards under Title III and either the 1991 Standards or the Uniform Federal Accessibility Standards under Title II.
} 
provider and supplier types by the final rule, "Medicare and Medicaid Programs; Fire Safety Requirements for Certain Health Care Facilities" published on May 4, 2016 (81 FR 26872).

The materials that are incorporated by reference can be found for interested parties and are available for inspection at the CMS Information Resource Center, 7500 Security Boulevard, Baltimore, MD 21244, or from the National Fire Protection Association, 1 Batterymarch Park, Quincy, MA 02269. If any changes to these editions of the Codes are incorporated by reference, CMS will publish a document in the Federal Register to announce those changes.

The 2012 edition of the NFPA 101 (including the TIAs) provides minimum requirements, with due regard to function, for the design, operation and maintenance of buildings and structures for safety to life from fire. Its provisions also aid life safety in similar emergencies.

The 2012 edition of the NFPA 99 (including the TIAs) provides minimum requirements for health care facilities for the installation, inspection, testing, maintenance, performance, and safe practices for facilities, material, equipment, and appliances, including other hazards associated with the primary hazards.

\section{Ambulatory Health Care Occupancies}

According to our memorandum, "Survey \& Certification: 13-47-LSC/ ESRD," issued July 12, 2013, dialysis facilities that are subject to the LSC provisions must meet the requirements of the Ambulatory Health Care Occupancy chapters 20 and 21 of the LSC. Dialysis facilities that are not subject to our LSC regulations must continue to meet State and local fire codes. (See https://www.cms.gov/ Medicare/Provider-Enrollment-andCertification/

SurveyCertificationGenInfo/Downloads/ Survey-and-Cert-Letter-13-47.pdf.)

The following are key provisions in the 2012 edition of the LSC from Chapter 20, "New Ambulatory Health Care Occupancies”, and Chapter 21, "Existing Ambulatory Health Care Occupancies." We have provided the LSC citation and a description of the requirement.

The 2012 edition of the LSC defines an "Ambulatory Health Care Occupancy" as a facility capable of treating 4 or more patients simultaneously on an outpatient basis. We believe that dialysis facilities that do not provide one or more exits to the outside at grade level from the patient treatment area should also be required to meet the provisions applicable to Ambulatory Health Care Occupancy Chapters, regardless of the number of patients served, as a matter of health and safety of patients receiving services in these facilities. In the burden reduction final rule, published in the Federal Register on May 12, 2012 entitled, "Medicare and Medicaid Program; Regulatory Provisions to Promote Program Efficiency,

Transparency, and Burden Reduction" (77 FR 29002), we removed the provision's applicability to dialysis facilities with at-grade exits directly from the treatment area because, in our view, there was, and continues to be, an extremely low risk of fire in dialysis facilities. Medicare-approved dialysis facilities that provide exits to the outside at grade level would continue to be required to follow State and local fire codes, which we believe provide for sufficient patient protection in the event of an emergency. If a facility's exits were located above or below grade, patients would require more time to evacuate. Consequently, we believe that the LSC would still be required due to the additional risk entailed in longer exit times.

Sections 20.3.2.1 and 21.3.2.1-Doors

This provision requires all doors to hazardous areas be self-closing or close automatically.

Sections 20.3.2.6 and 21.3.2.6-Alcohol Based Hand Rubs

This provision explicitly allows aerosol dispensers, in addition to gel hand rub dispensers. The aerosol dispensers are subject to limitations on size, quantity, and location, just as gel dispensers are limited. Automatic dispensers are also now permitted in ambulatory care facilities, provided, among other things, that-(1) they do not release contents unless they are activated; (2) the activation occurs only when an object is within 4 inches of the sensing device; (3) any object placed in the activation zone and left in place must not cause more than one activation; (4) the dispenser must not dispense more than the amount required for hand hygiene consistent with the label instructions; (5) the dispenser is designed, constructed and operated in a way to minimize accidental or malicious dispensing; and (6) all dispensers are tested in accordance with the manufacturer's care and use instructions each time a new refill is installed. The provision further defines prior language regarding "above or adjacent to an ignition source" as being "within 1 inch" of the ignition source.
Sections 20.3.5 and 21.3.5Extinguishment Requirements

This provision is related to sprinkler system requirements and requires the evacuation of a building or the instituting of an approved fire watch when a sprinkler system is out of service for more than 10 hours in a 24hour period until the system has been returned to service. A facility must evacuate the building or portion of the building affected by the system outage until the system is back in service, or establish a fire watch until the system is back in service.

\section{2012 Edition of the Health Care Facilities Code}

The 2012 edition of the NFPA 99, “Health Care Facilities Code," addresses requirements for both health care occupancies and ambulatory care occupancies, and serves as a resource for those who are responsible for protecting health care facilities from fire and associated hazards. The purpose of this Code is to provide minimum requirements for the installation, inspection, testing, maintenance, performance, and safe practices for health care facility materials, equipment and appliances. This Code is a compilation of documents that have been developed over a 40-year period by NFPA, and is intended to be used by those persons involved in the design, construction, inspection, and operation of health care facilities, and in the design, manufacture, and testing of appliances and equipment used in patient care areas of health care facilities. It provides information on subjects, for example, medical gas and vacuum systems, electrical systems, electrical equipment, and gas equipment. The NFPA 99 applies specific requirements in accordance with the results of a risk-based assessment methodology. A risk-based approach allows for the application of requirements based upon the types of treatment and services being provided to patients or residents rather than the type of facility in which they are being performed. In order to ensure the minimum level of protection afforded by NFPA 99 is applicable to all patient and resident care areas within a health care facility, we proposed adoption of the 2012 edition of NFPA 99, with the exception of chapters 7-“"Information Technology and Communications Systems for Health Care Facilities"; 8"Plumbing"; 12-"Emergency Management"; and 13-“Security Management". The first three chapters of the NFPA 99 address the administration of the NFPA 99, the 
referenced publications, and definitions. Short descriptions of some of the more important provisions of NFPA 99 follow:

\section{Chapter 4-Fundamentals}

Chapter 4 provides guidance on how to apply NFPA 99 requirements to health care facilities based upon "categories" determined when using a risk-based methodology.

There are four categories utilized in the risk assessment methodology, depending on the types of treatment and services being provided to patients or residents. Section 4.1.1 of NFPA 99 describes Category 1 as, "Facility systems in which failure of such equipment or system is likely to cause major injury or death of patients or caregivers. . . ." Section A.4.1.1 provides examples of what a major injury could include, such as amputation or a burn to the eye. Section 4.1.2 describes Category 2 as, "Facility systems in which failure of such equipment is likely to cause minor injury to patients or caregivers. . . ." Section A.4.1.2 describes a minor injury as one that is not serious or involving risk of life. Section 4.1.3 describes Category 3 as, "Facility systems in which failure of such equipment is not likely to cause injury to patients or caregivers, but can cause patient discomfort. . . ." Section 4.1.4 describes Category 4 as, "Facility systems in which failure of such equipment would have no impact on patient care. . . ."

Section 4.2 requires that each facility that is a health care or ambulatory occupancy define its risk assessment methodology, implement the methodology, and document the results. We did not propose to require the use of any particular risk assessment procedure. However, if future situations indicate the need to define a particular risk assessment procedure, we would pursue that through a separate notice and comment rulemaking.

\section{Chapter 5-Gas and Vacuum Systems}

The hazards addressed in Chapter 5 include the ability of oxygen and nitrous oxide to exacerbate fires, safety concerns from the storage and use of pressurized gas, and the reliance upon medical gas and vacuum systems for patient care. Chapter 5 does not mandate the installation of any systems; rather, if they are installed or are required to be installed, the systems will be required to comply with NFPA 99. Chapter 5 covers the performance, maintenance, installation, and testing of the following:
- Non-flammable medical gas systems with operating pressure below a gauge pressure of 300 psi;

- Vacuum systems in health care facilities;

- Waste anesthetic gas disposal systems (WAGD); and

- Manufactured assemblies that are intended for connection to the medical gas, vacuum, or WAGD systems.

\section{Chapter 6-Electrical Systems}

The hazards addressed in Chapter 6 are related to the electrical power distribution systems in health care facilities, and address issues such as electrical shock, power continuity, fire, electrocution, and explosions that might be caused by faults in the electrical system. Chapter 6 also covers the performance, maintenance, and testing of the electrical systems in health care facilities.

Chapter 9-Heating, Ventilation, and Air Conditioning (HVAC)

Chapter 9 requires HVAC systems serving spaces or providing health care functions to be in accordance with the American Society of Heating, Refrigeration and Air-Conditioning Engineers (ASHRAE) Standard 170Ventilation of Health Care Facilities (2008 edition) (http://www.ashrae.org)

Chapter 9 does not apply to existing

HVAC systems, but applies to the construction of new health care facilities, and the altered, renovated, or modernized portions of existing systems or individual components. Chapter 9 ensures minimum levels of heating, ventilation and air conditioning performance in patient and resident care areas. Some of the issues discussed in Chapter 9 are as follows:

- HVAC system energy conservation;

- Commissioning;

- Piping;

- Ductwork;

- Acoustics;

- Requirements for the ventilation of medical gas storage and trans-filling areas;

- Waste anesthetic gases;

- Plumes from medical procedures;

- Emergency power system rooms; and

- Ventilation during construction.

Chapter 10-Electrical Equipment

Chapter 10 covers the performance, maintenance, and testing of electrical equipment in health care facilities. Much of this chapter applies to requirements for portable electrical equipment in health care facilities, but there are also requirements for fixedequipment and information on administrative issues.
Chapter 11-Gas Equipment

The hazards addressed in Chapter 11 relate to general fire, explosions, and mechanical issues associated with gas equipment, including compressed gas cylinders.

\section{Chapter 14-Hyperbaric Facilities}

Chapter 14 addresses the hazards associated with hyperbaric facilities in health care facilities, including electrical, explosive, implosive, as well as fire hazards. Chapter 14 sets forth minimum safeguards for the protection of patients and personnel administering hyperbaric therapy and procedures. Chapter 14 contains requirements for hyperbaric chamber manufacturers, hyperbaric facility designers, and personnel operating hyperbaric facilities. It also contains requirements related to construction of the hyperbaric chamber itself and the equipment used for supporting the hyperbaric chamber, as well as administration and maintenance. Many requirements in this chapter are applicable only to new construction and new facilities.

Chapter 15-Features of Fire Protection

Chapter 15 covers the performance, maintenance, and testing of fire protection equipment in health care facilities. Issues addressed in this chapter range from the use of flammable liquids in an operating room to special sprinkler protection. These fire protection requirements are independent of the risk-based approach, as they are applicable to all patient care areas in both new and existing facilities.

Chapter 15 has several sections taken directly from the NFPA 101, including requirements for the following:

- Construction and

compartmentalization of health care facilities.

- Laboratories.

- Utilities.

- Heating, ventilation and air conditioning systems.

- Elevators.

- Escalators.

- Conveyors.

- Rubbish Chutes.

- Incinerators.

- Laundry Chutes.

- Fire detection, alarm and communication systems.

- Automatic sprinklers and other extinguishing equipment.

- Compact storage including mobile storage and maintenance.

- Testing of water based fire protection systems.

These sections have requirements for inspection, testing and maintenance which apply to all facilities, as well as 
specific requirements for existing systems and equipment that also apply to all facilities.

- The prospective timeline for applicability of these requirements would be 60 days after the publication of the final rule in the Federal Register. We solicited comments on the proposal of the adoption of the 2012 NFPA 101 and the 2012 NFPA 99 for dialysis facilities that do not provide one or more exits to the outside at grade level from the treatment area level in the proposed rule "Fire Safety Requirements for Certain Dialysis Facilities," published November, 4, 2016 (81 FR 76899).

We received 4 comments and all commenters were in support of the proposal. Therefore, we are finalizing the adoption of the 2012 NFPA 101 and the 2012 NFPA 99 for dialysis facilities that do not provide one or more exits to the outside at grade level from the treatment area level.

\section{Technical Correction}

We inadvertently left out the update of $\S 494.60(d)(2)$ from the 2000 edition of the Life Safety Code to the 2012 edition of the Life Safety Code. This update goes along with the overall adoption of the 2012 edition of the Life Safety Code. This will have no impact on facilities as they are all already meeting the 2012 edition of the Life Safety Code in accordance with state and local requirements.

\section{Collection of Information Requirements}

This document does not impose information collection requirements, that is, reporting, recordkeeping or third-party disclosure requirements. Consequently, there is no need for review by the Office of Management and Budget under the authority of the Paperwork Reduction Act of 1995 (44 U.S.C. 3501 et seq.).

\section{Final Rule: Hospital and Critical Access Hospital (CAH) Changes To Promote Innovation, Flexibility, and Improvement in Patient Care}

\section{A. Background}

On June 16, 2016, we published a proposed rule in the Federal Register, "Medicare and Medicaid Programs; Hospital and Critical Access Hospital (CAH) Changes To Promote Innovation, Flexibility, and Improvement in Patient Care”" (81 FR 39447), to revise a number of hospital and $\mathrm{CAH}$ requirements, including those focused on infection control, antibiotic use, and antidiscrimination. We are now finalizing several of the proposed changes in order to modernize the hospital and critical access hospital (CAH) requirements, improve quality of care, and support HHS and CMS priorities. We believe that benefits of these finalized requirements will include: Reduced incidence of hospitalacquired conditions (HACs), including reduced incidence of healthcareassociated infections (HAIs); reduced inappropriate antibiotic use; reduced regulatory burden and increased cost savings for hospitals, CAHs, and insurers; and strengthened patient protections overall. Specifically, we proposed to revise the conditions of participation (CoPs) for hospitals and CAHs to address:

- Discriminatory behavior by healthcare providers that may create real or perceived barriers to care;

- A requirement regarding a patient's right to access his or her own medical records, including in an electronic format;

- Continued use of the term "Licensed Independent Practitioners" (LIPs), which might inadvertently exacerbate workforce shortage concerns, might unnecessarily impose regulatory burden on hospitals by restricting a hospital's ability to allow non-physician practitioners such as physician assistants (PAs) to operate within the scope of practice allowed by state law, and does not recognize the benefits to patient care that might be derived from fully utilizing PAs and their clinical skills to the highest levels of their training, education, and experience as allowed by hospital policy in accordance with state law;

- The use of quality reporting program data by hospital Quality Assessment and Performance Improvement (QAPI) programs;

- Requirements in the Nursing services CoP to improve clarity and provide some regulatory flexibility and burden relief;

- Requirements in the Medical records services CoP to improve clarity regarding the distinctions between a patient's inpatient and outpatient status and the subtle differences between certain aspects of medical record documentation related to each status;

- Requirements that do not fully conform to current standards for infection control for both hospitals and CAHs;

- Requirements for antibiotic stewardship programs to help reduce inappropriate antibiotic use and antimicrobial resistance for both hospitals and CAHs;

- A requirement for CAHs that would allow a patient's nutritional needs to be met in accordance with recognized dietary practices and the orders of the practitioner responsible for the care of the patients, or by a qualified dietician or qualified nutrition professional as authorized by the medical staff in accordance with State law; and

- Requirements for CAHs to establish a quality assessment and performance improvement program (QAPI).

\section{B. Provisions of the Proposed} Regulations and Responses to Public Comments for Hospitals (42 CFR Part 482)

\section{General Comments}

In response to the proposed rule, we received 200 public comments. Commenters included individuals, healthcare professionals and corporations, national associations and coalitions, state health departments, patient advocacy organizations, and individual facilities that would be impacted by the regulation.

Generally, most comments expressed support for the regulatory changes. We have provided a summary of the public comments, our responses to those comments, and any changes made as a result of those comments in the proceeding sections. Several commenters expressed concern that we underestimated the time and effort required for compliance with the antibiotic stewardship and QAPI requirements, especially for smaller hospitals, including CAHs. Commenters requested a delayed implementation for these requirements.

\section{Implementation Timeframe}

Comment: We received several comments stating that we have underestimated the time necessary to implement some of the requirements contained in this rule. Some commenters stated that the proposed hospital and CAH infection control and antibiotic stewardship and QAPI provisions required additional time to implement. These commenters requested that we grant additional time for the implementation for these requirements. Commenters cited challenges associated with implementing these requirements, especially for small, rural hospitals and CAHs including obtaining and training appropriate staff for the required positions.

Response: We understand the complexities of the required changes in this rule for hospitals and CAHs, particularly the effects on CAHs and small, rural hospitals. As a result, we are using the following implementation schedule for the provisions of this final rule: 
- CAH QAPI requirements-an implementation date that is 18 months after the effective date of this final rule;

- Hospital and CAH compliance with the antibiotic stewardship requirements-an implementation date that is six months from the effective date of this final rule; and

- All other requirements, including those for patient's rights-an implementation date that is 60 days from the publication of this final rule.

\section{Non-Discrimination}

We proposed to establish at $\S 482.13(\mathrm{i})$ for hospitals and $\S 485.635$ (g) for CAHs, explicit requirements that a hospital (or CAH) not discriminate on the basis of race, color, religion, national origin, sex (including gender identity), sexual orientation, age, or disability and that the hospital (or CAH) establish and implement a written policy prohibiting discrimination on the basis of race, color, religion, national origin, sex (including gender identity), sexual orientation, age, or disability.

We proposed to further require that each patient, (and/or support person, where appropriate), is informed, in a language he or she can understand, of the right to be free from discrimination against them on any of these bases when he or she is informed of his or her other rights under § 482.13(i) (or § 485.635(g)). In addition, we proposed to require that the hospital (or CAH) inform the patient and/or representative, and/or support person, on how he or she can seek assistance if they encounter discrimination.

Comment: We received numerous comments that expressed support for this proposal and also discussed the potential benefits of the proposal to patients. In addition, we received comments that expressed concern about the consequences of the implementation of this proposal and suggested modifications to our proposed requirement. Commenters also discussed potential technical difficulties that may exist when implementing this proposal and they expressed concern that the proposed requirement may be duplicative of other current federal requirements.

Response: In response to these comments, we are not finalizing the proposal to require explicit nondiscrimination requirements in the CoPs and we are instead deferring to the nondiscrimination requirements of Section 1557 of the Affordable Care Act.

Final Action: We are not finalizing proposed § 482.13(i) and § 485.635(g).

\section{Licensed Independent Practitioner}

We proposed to delete the modifying term "independent" from the CoPs at $\S 482.13(\mathrm{e})(5)$, as well as at $\S 482.13(\mathrm{e})(8)(\mathrm{ii})$, and also proposed to revise the provision to be in keeping with the language of the Children's Health Act of 2000 (Pub. L. 106-310) (CHA) regarding restraint and seclusion orders and licensed practitioners. Therefore, we proposed that $\S 482.13(\mathrm{e})(5)$ read that the use of restraint or seclusion must be in accordance with the order of a physician or other licensed practitioner who is responsible for the care of the patient and authorized to order restraint or seclusion by hospital policy in accordance with State law. We proposed that $\S 482.13(\mathrm{e})(8)(\mathrm{ii})$ would state that, after 24 hours, before writing a new order for the use of restraint or seclusion for the management of violent or selfdestructive behavior, a physician or other licensed practitioner who is responsible for the care of the patient and authorized to order restraint or seclusion by hospital policy in accordance with State law would have to see and assess the patient.

We proposed to revise the provisions in sections $\S 482.13(\mathrm{e})(10)$,

$\S 482.13(\mathrm{e})(11), \S 482.13(\mathrm{e})(12)(\mathrm{i})(\mathrm{A})$, $\S 482.13(\mathrm{e})(14)$, and $\S 482.13(\mathrm{~g})(4)(\mathrm{ii})$ that contain the term "licensed independent practitioner" by changing the term from "licensed independent practitioner" to simply "licensed practitioner." We also proposed to remove the term "physician assistant" from the current provisions at $\S 482.13(\mathrm{e})(12)(\mathrm{i})(\mathrm{B})$ and $\S 482.13(\mathrm{e})(14)$.

Comment: The majority of commenters were supportive of this change. Specifically, commenters noted that the proposed language change will remove uncertainty regarding these provisions and clearly demonstrates that Physician Assistants (PAs) are authorized to order restraint and seclusion, in accordance with state law and facility policy, when medically necessary to protect patients and health professionals. One commenter did not support the removal of the term "independent" from this requirement. The commenter stated that removing the term "independent" may make this requirement applicable to other care providers, such as registered nurses.

Response: We thank commenters for their support of this requirement. We believe this revision reflects our goal to have health professionals operate within the scope of practice allowed by state law, and recognizes the need to fully utilize the healthcare workforce. We also believe that this change will reduce unnecessary burden for hospitals and remove obstacles PAs face when ordering seclusion and restraints. We disagree with the commenters who stated that the removal of the term "independent" will cause confusion over the applicability of this requirement. Our proposed removal of the term "independent" is consistent with the language used in the CHA, which utilizes the term "other licensed practitioner", without the independent modifying term. In addition, the order of restraint or seclusion must be ordered by a licensed practitioner who is authorized by hospital policy in accordance with State law to do so. This would exclude Registered Nurses or other hospital staff, who either through State law or hospital policy, would not have the authorization to order the use of restraints and seclusion.

After consideration of the comments we received, we are finalizing this proposal, without modification.

Final Action: We are finalizing the following revisions to $\S 482.13$ :

1 . Remove the modifying term "independent" from the CoPs at $\S 482.13(\mathrm{e})(5)$ and $\S 482.13(\mathrm{e})(8)(\mathrm{ii})$.

2 . Revise $\S 482.13(\mathrm{e})(5)$ to state that the use of restraint or seclusion must be in accordance with the order of a physician or other licensed practitioner who is responsible for the care of the patient and authorized to order restraint or seclusion by hospital policy in accordance with State law.

3 . Revise the provisions in sections § 482.13(e)(10), § 482.13(e)(11), $\S 482.13(\mathrm{e})(12)(\mathrm{i})(\mathrm{A}), \S 482.13(\mathrm{e})(14)$, and $\S 482.13(\mathrm{~g})(4)(\mathrm{ii})$ that contain the term "licensed independent practitioner" by changing the term to simply "licensed practitioner."

4. Remove the term "physician assistant" from the current provisions at § 482.13(e)(12)(i)(B) and § 482.13(e)(14).

5. Quality Assessment and Performance Improvement (QAPI) Program (§ 482.21)

We proposed a minor change to the program data requirements at $\S 482.21$ (b), which would require that the hospital QAPI program incorporate quality indicator data including patient care data submitted to or received from quality reporting and quality performance programs, including but not limited to data related to hospital readmissions and hospital-acquired conditions

Comment: We received mostly positive feedback regarding this requirement; however, some commenters asked that we remove the provided example of "data related to hospital readmissions and hospitalacquired conditions." Commenters 
believed that the inclusion of this information makes it unclear to hospitals that they should utilize all data available to them. One commenter also disagreed with any proposal that would restrict quality improvement work to a limited number of areas.

Response: We thank the commenters for their feedback. We believe that this requirement affords hospitals increased flexibility, while continuing to promote patient safety and quality of care. As revised by this final rule, the regulation at $\S 482.21$ (b)(1) now requires that the QAPI program “incorporate quality indicator data including patient care data, and other relevant data such as data submitted to or received from Medicare quality reporting and quality performance programs, including but not limited to data related to hospital readmissions and hospital-acquired conditions." We believe the intent of the regulation is clear as the language states that the data that must be incorporated is not limited to data related to hospital readmissions and hospital-acquired conditions; however, we will ensure that the intent is also clear in the Interpretive Guidelines for this requirement. Note that CMS historically releases Interpretive Guidelines for new regulations after the final rule has been published. Furthermore, we note that these requirements would not restrict hospitals to a certain number of quality improvement areas, but they are instead minimum requirements that hospitals can choose to exceed as they strive to improve the quality of the services that they provide.

Final Action: We are finalizing $\S 482.21(\mathrm{~b})$ as proposed.

\section{Nursing Services (482.23)}

As a result of our internal review of the CoPs for nursing services, we recognized that some of our requirements might be ambiguous and confusing due to unnecessary distinctions between inpatient and outpatient services, or might fail to account for the variety of ways through which a hospital might meet its nurse staffing requirements. We proposed to make revisions to the nursing services CoP to improve clarity. Specifically, we proposed to revise $\S 482.23(\mathrm{~b})$, which currently states that there must be supervisory and staff personnel for each department or nursing unit to ensure, when needed, the immediate availability of a registered nurse for bedside care of any patient. We proposed to delete the term "bedside," which might imply only inpatient services to some readers. The nursing service would have to ensure that patient needs were being met by ongoing assessments of patients' needs and would have to provide nursing staff to meet those needs regardless of whether the patient was an inpatient or an outpatient. There would have to be sufficient numbers and types of supervisory and staff nursing personnel to respond to the appropriate nursing needs and care of the patient population of each department or nursing unit. When needed, a registered nurse would have to be available to care for any patient. We understand that the term "immediate availability" has been interpreted to mean physically present on the unit or in the department. We further understand that there are some outpatient services where it might not be necessary to have a registered nurse physically present. For example, while it is clearly necessary to have an RN present in an outpatient ambulatory surgery recovery unit, it might not be necessary to have an $\mathrm{RN}$ on-site at a hospital MRI facility that is outside the hospital building, but still on the hospital campus. We proposed to allow a hospital to establish a policy that would specify which, if any, outpatient departments would not be required to have an RN physically present as well as the alternative staffing plans that would be established under such a policy. We would require such a policy to take into account factors such as the services delivered, the acuity of patients typically served by the facility, and the established standards of practice for such services. In addition, we would propose that the policy must be approved by the medical staff and be reviewed at least once every three years. We solicited comments on the need for, the risks of establishing, and the appropriate criteria we should require for such an exception.

We also proposed to clarify in (b)(4) (which currently requires that the hospital must ensure that the nursing staff develops, and keeps current, a nursing care plan for each patient and that the plan may be part of an interdisciplinary care plan) that while a nursing care plan was needed for every patient, the care plan would be expected to reflect the needs of the patient and the nursing care to be provided to meet those needs. The care plan for a patient with complex medical needs and a longer anticipated hospitalization would be more extensive and detailed than the care plan for a patient with a less complex medical need expecting only a brief hospital stay. We expect that a nursing care plan would be initiated and implemented in a timely manner, include patient goals as part of the patient's nursing care assessment and, as appropriate, physiological and psychosocial factors (such as specific physical limitations and available support systems), physical and behavioral health comorbidities, and patient discharge planning. In addition, it would have to be consistent with the plan for the patient's medical care and demonstrate evidence of reassessment of the patient's nursing care needs, response(s) to nursing interventions, and, as needed, revisions to the plan.

Finally, we proposed to revise (b)(6) (which currently states that nonemployee licensed nurses working in the hospital must adhere to the policies and procedures of the hospital and that the director of nursing service must provide for the adequate supervision and evaluation of the clinical activities of non-employee nursing personnel) to clarify that all licensed nurses who provide services in the hospital must adhere to the policies and procedures of the hospital. In addition, the director of nursing service must provide for the adequate supervision and evaluation of the clinical activities of all nursing personnel (that is, all licensed nurses and any non-licensed personnel such as nurse aides, orderlies, or other nursing support personnel who are under the direction of the nursing service) which occur within the responsibility of the nursing service, regardless of the mechanism through which those personnel were obtained. We recognize that there are a variety of arrangements under which hospitals obtain the services of licensed nurses. Mechanisms may include direct employment, the use of contract or agency nurses, a leasing agreement, volunteer services or some other arrangement. No matter how the services of a licensed nurse were obtained, in order to ensure the health and safety of patients, all nurses would have to know and adhere to the policies and procedures of the hospital and there must be adequate supervision and evaluation of the clinical activities of all nursing personnel who provide services that occur within the responsibility of the nursing service. We would expect non-licensed personnel to be supervised by a licensed nurse.

In addition, we proposed to delete inappropriate references to $\S 482.12$ (c) that are currently in (c)(1) and (3). We discuss these technical corrections in detail below.

Comment: Commenters expressed concern regarding the removal of the word "bedside" under § 482.23(b), (which stated that there must be supervisory and staff personnel for each department or nursing unit to ensure, when needed, the immediate availability of a registered nurse for 
bedside care of any patient). Commenters noted that our proposed revision could create confusion in certain inpatient departments and asked that CMS clarify that each hospital department or nursing unit should ensure that nurse staffing should be immediately available, when needed. Commenters also asked that we clarify that policies related to nurse staffing are approved by the hospital's medical staff in conjunction with nursing leadership. One commenter stated that the approval of any policies regarding nursing services would be under the authority of the hospital's director of nursing and medical staff approval would not be needed as proposed here.

Response: The nursing service must ensure that patient needs are met by continuously assessing the needs of patients and must provide nursing staff to meet those needs, regardless of whether the patient is an inpatient or an outpatient. There must be sufficient numbers and types of supervisory and staff nursing personnel to meet the nursing needs and to care for the patient population of each department or nursing unit. A registered nurse must be available to care for any patient, as determined by the needs of the patient and hospital policy. Note that the term "immediate availability" has been interpreted to mean physically present on the unit or in the department. Also note that there are some outpatient services where it might not be necessary to have a registered nurse physically present. For example, while it is clearly necessary to have an RN present in an outpatient ambulatory surgery recovery unit, it might not be necessary to have an RN on-site at an off-campus outpatient department where radiology services are offered. Hospitals are provided the flexibility to establish a policy that would specify which, if any, outpatient departments would not be required to have an $\mathrm{RN}$ physically present as well as the alternative staffing plans that would be established under such a policy. Such a policy must take into account factors such as the services delivered, the acuity of patients typically served by the facility, and the established standards of practice for such services. We agree with the comment that stated that the approval of any nursing services policy falls under the authority of the hospital's nursing leadership and we have modified the proposed requirement at

$\S 482.23(\mathrm{~b})(7)(\mathrm{iii})$ to reflect that in this final rule.

Comment: We received positive comments about the requirement under $\S 482.23(\mathrm{~b})(4)$, which requires that the nursing care plan, which is needed for every patient, reflect the needs of the patient and the nursing care to be provided to meet those needs. Commenters stated these changes help ensure that the clinical team is working together with the patient and the patient's family to ensure that the team is continuously working towards meeting the established patient goals. However, as evidenced by some comments, there appears to exist some confusion over whether a nursing care plan is required for both inpatients and outpatients or if it is required for inpatients only.

Response: We appreciate the positive feedback received for this requirement. Initiating a nursing care plan for patients that reflects the needs of the patient will lead to better patient outcomes and has the potential to decrease length of stay.

Regarding the question of which patients (all patients or only inpatients) are required to have a nursing care plan, we must look at both the regulatory text and the interpretive guidance contained in the SOM, Appendix A, Section A0396, for this provision. While the actual regulatory text has always simply used the term, "patient," implying both inpatients and outpatients, other areas of the CoPs specifically use the term "inpatient" as does the language of the Act (specifically with regard to nursing services) as well as other instances in the CoPs that refer to patient "admissions," which further implies inpatients. Additionally, the interpretive guidance for this provision in the SOM, Appendix A, has traditionally held that the requirement for a nursing care plan only applies to patients after their "admission," (that is, inpatients only) (https://www.cms.gov/ Regulations-and-Guidance/Guidance/ Manuals/Downloads/som107ap_a_ hospitals.pdf, p. 224).

While we believe that nursing care plans most appropriately, and in most instances, apply only to inpatients, we urge hospitals to review their policies and procedures in this area to determine if there are outpatients where a nursing care plan would be appropriate and should be required for the benefit of the patient's health and safety and for improved outcomes. For instance, hospitals should look at the policies that they develop for the provisions that we are finalizing here, at $\S 482.23(\mathrm{~b})(7)$, regarding those policies and procedures that must be in place to establish which outpatient departments, if any, are not required under hospital policy to have a registered nurse present. As we discussed previously, that while it is clearly necessary to have an RN present in an outpatient ambulatory surgery recovery unit, it might not be necessary to have an RN on-site at a hospital MRI facility that is outside the hospital building, but still on the hospital campus. In exercising this policy flexibility provided in this final rule for reviewing the need for establishing which outpatient units must have an RN present for patient care and safety, we likewise encourage hospitals to exercise a similar regulatory flexibility in reviewing their policies for establishing which types of outpatients would require a nursing care plan through a similar lens-that is, based on the services that a patient is receiving and the location in which he or she is receiving those services. We further believe that the example provided here regarding the requirement differences in the patient's needs for having an RN present, which clearly exist between an outpatient undergoing ambulatory surgery and one receiving an MRI or other radiologic services, is entirely relevant to the considerations for determining which patient needs a nursing care plan.

Comment: We received positive feedback regarding $\S 482.23(\mathrm{~b})(6)$, in which we proposed to clarify that all licensed nurses who provide services in the hospital must adhere to the policies and procedures of the hospital and addresses the supervision and evaluation of the clinical activities of all nursing personnel. Commenters appreciated the clarification of the requirements in this in calling for adequate supervision and evaluation of all nursing personnel. One commenter asked that we clarify that nursing leadership is responsible for ensuring that there are clear lines of reporting and supervision.

Response: We appreciate the comments received on the proposed requirement. We expect all nursing personnel to have a clear understanding of the reporting and supervisory structure and it is the responsibility of nursing leadership to ensure that there are clear lines of reporting and supervision. This requirement must be met regardless of the employment type or status of the nursing personnel, including but not limited to those employed via direct employment, the use of contract or agency nurses, a leasing agreement, volunteer services or some other arrangement. No matter how the services of a licensed nurse are obtained, in order to ensure the health and safety of patients, all nurses must know and adhere to the policies and procedures of the hospital and there must be adequate supervision and evaluation of the clinical activities of all nursing personnel who provide services 
that occur within the responsibility of the nursing service. We would expect non-licensed personnel to be supervised by a licensed nurse.

Final Decision: After consideration of the comments we received on the proposed rule, we are finalizing $§ 482.23$ as proposed with the exception of the proposed requirement at $\S 482.23(\mathrm{~b})(7)(\mathrm{iii})$, which we have revised in response to comments by replacing "medical staff” with "director of nursing," and which we are finalizing here.

\section{Medical Record Services (§ 482.24)}

We proposed to revise $\S 482.24$ (c) to require that the content of the medical record contain information to justify all admissions and continued hospitalizations, support the diagnoses, describe the patient's progress and responses to medications and services, and document all inpatient stays and outpatient visits to reflect all services provided to the patient.

Similarly, we proposed to revise $\S 482.24(\mathrm{c})(4)$ (ii) to include "all diagnoses specific to each inpatient stay and outpatient visit," which would include specifying any admitting diagnoses. At § 482.24(c)(4)(iv), we proposed to require that the content of the record include documentation of complications, hospital-acquired conditions, healthcare-associated infections, and adverse reactions to drugs and anesthesia. We also propose changes to $\S 482.24$ (c)(4)(vi) to add "progress notes. . . interventions, responses to interventions . . ." to the required documentation of "practitioners' orders" to emphasize the necessary documentation for both inpatients and outpatients. We also proposed to add the phrase "to reflect all services provided to the patient," so that the entire provision would now read that the content of the record must contain all practitioners' progress notes and orders, nursing notes, reports of treatment, interventions, responses to interventions, medication records, radiology and laboratory reports, and vital signs and other information necessary to monitor the patient's condition and to reflect all services provided to the patient.

We proposed to change $\S 482.24$ (c)(4)(vii) to require that all patient medical records document discharge and transfer summaries with outcomes of all hospitalizations, disposition of cases, and provisions for follow-up care for all inpatient and outpatient visits to reflect the scope of all services received by the patient.

Finally, we proposed to revise $\S 482.24$ (c)(4)(viii) so that the content of the medical record would contain final diagnoses with completion of medical records within 30 days following all inpatient stays, and within 7 days following all outpatient visits.

Comment: The comments we received on these proposed changes were concerned that the medical records documentation revisions would be unduly burdensome and confusing regarding distinctions between the requirements for inpatients versus outpatients. Commenters also expressed concerns over the ongoing interplay between EHRs and paper-based records systems and EHR interoperability issues that may arise.

Response: We appreciate the commenter's feedback regarding these proposals. We agree that the proposed changes to the medical records documentation requirements would impose an additional undue burden on hospitals and we are therefore not finalizing this proposal at this time.

Final Action: Based on the public comments, we are not finalizing the proposed changes to the Medical Records requirements at $\S 482.24$.

8. Infection Prevention and Control and Antibiotic Stewardship Programs (§ 482.42)

We proposed a change to the title of this CoP to "Infection prevention and control and antibiotic stewardship programs.” By adding the word "prevention" to the CoP name, our intent is to promote larger, cultural changes in hospitals such that prevention initiatives are recognized on balance with their current, traditional control efforts. And by adding "antibiotic stewardship" to the title, we would emphasize the important role that a hospital should play in combatting antimicrobial resistance through implementation of a robust stewardship program that follows nationally recognized guidelines for appropriate antibiotic use. Along with these changes, we proposed to change the introductory paragraph to require that a hospital's infection prevention and control and antibiotic stewardship programs be active and hospital-wide for the surveillance, prevention, and control of HAIs and other infectious diseases, and for the optimization of antibiotic use through stewardship. We would also require that a program demonstrate adherence to nationally recognized infection prevention and control guidelines for reducing the transmission of infections, as well as best practices for improving antibiotic use where applicable, and for reducing the development and transmission of HAIs and antibiotic-resistant organisms.
While these particular changes are new to the regulatory text, it is worth noting that these requirements, with the exception of the new requirement for an antibiotic stewardship program, have been present in the Interpretive Guidelines for hospitals since 2008 (See A0747 at Appendix A-Survey Protocol, Regulations and Interpretive Guidelines for Hospitals, http://cms.gov/manuals/ Downloads/som107ap_a_hospitals.pdf).

We also proposed to introduce the term "surveillance" into the text of the regulation. The addition of this term, which is also already in use in CMS Interpretive Guidelines for hospitals, is being proposed to bring the regulation up to date by reflecting current terminology in the field. As has been described in the Interpretive Guidelines for this regulation, "surveillance" includes infection detection, data collection, and analysis; monitoring; and evaluation of preventive interventions. (See SOM, Appendix ASurvey Protocol, Regulations and Interpretive Guidelines for Hospitals, pp. 361-362, http://cms.gov/manuals/ Downloads/som107ap_a_hospitals.pdf). In collaboration with the hospital's QAPI program, the hospital would be required to develop and implement appropriate infection prevention and control interventions to address issues identified through its detection activities.

We also proposed a new requirement that hospitals demonstrate adherence to nationally recognized infection prevention and control guidelines, as well as best practices for improving antibiotic use where applicable, and for reducing the development and transmission of HAIs and antibioticresistant organisms. We realize that, in developing the patient health and safety requirements that are the hospital CoPs, particular attention must be paid to the ever-evolving nature of medicine and patient care. Moreover, a certain degree of latitude must be left in the requirements to allow for innovations in medical practice that improve the quality of care and move toward the reduction of medical errors and patient harm.

Therefore, we intentionally built flexibility into the revised regulations by proposing language that requires hospitals to demonstrate adherence to nationally recognized guidelines (and best practices where applicable) rather than requiring that all hospitals demonstrate adherence to a specific guideline or set of guidelines for infection prevention and control and for antibiotic stewardship. While the CDC guidelines and guidance (for example, guidelines from the Healthcare Infection 
Control Practices Advisory Committee (HICAPC) and Core Elements of Hospital Antibiotic Stewardship Programs) represent one set, there are other sets of nationally recognized guidelines from which hospitals might choose, such as those established by the Association for Professionals in Infection Control and Epidemiology (APIC), the Society for Healthcare Epidemiology of America (SHEA), and the Association of periOperative Registered Nurses (AORN). The U.S. Occupational Health and Safety Administration (OSHA) also issues federal regulations applicable to infection control practices. We believe this approach will provide hospitals the flexibility they need to select and integrate those standards that best suit their individual infection prevention and control and antibiotic stewardship programs. We also believe this approach will allow hospitals the flexibility to adapt their policies and procedures in concert with any updates in the guidelines they have elected to follow.

Comment: A few commenters were concerned that the proposed requirements for antibiotic stewardship programs would dictate the treatment options for patients with conditions such as Lyme disease. Some of these commenters were particularly concerned about the proposed rule's reference to IDSA antibiotic stewardship program guidelines.

Response: We proposed to intentionally build flexibility into the regulation by proposing language that requires hospitals to demonstrate adherence to nationally recognized guidance and guidelines, rather than any specific guidance, guideline, or set of guidelines, for best practices in infection prevention and control and for implementing antibiotic stewardship programs. For infection control best practices, CDC guidelines represent a fundamental set of practices, while other sets of nationally recognized infection control guidance and guidelines provide further setting- and procedure-specific options from which hospitals might choose, such as those established by APIC, SHEA, and IDSA. For the implementation of antibiotic stewardship programs, guidance is available from several organizations, including IDSA, SHEA, American Society for Health System Pharmacists, and CDC's Core Elements.

We appreciate the concerns expressed about the inclusion of guidelines developed by individual organizations, specifically, the Infectious Disease Society of America (IDSA). The intention in the proposed rule was to reference guidance for the implementation of antibiotic stewardship programs, not treatment guidelines for any particular infection. The reference to IDSA guidelines explicitly refers to guidelines for implementing stewardship programs and even references guidelines from other societies. These guidelines are referenced specifically because they are the only guidelines that we are aware of that are dedicated solely to the implementation of antibiotic stewardship programs in hospitals. We are not requiring that hospitals choose the IDSA guidelines for antibiotic stewardship programs specifically, but rather that they choose guidance on implementing antibiotic stewardship programs from a nationally recognized source.

Comment: One commenter recommended that rather than focusing on the explicit roles of two distinct staff, the CoPs instead focus on the overall process of clinical care and infection management and permit some flexibility in how hospitals establish each of their programs. They stated that in their experience, the ASP [antibiotic stewardship program] is part of the overall ICP [infection control program], which is broader than antibiotics.

Response: We agree that careful coordination between the infection prevention and control and antibiotic stewardship programs is essential and this is stated explicitly in the regulatory text. However, we believe it is also important to highlight the distinctions between the two programs. Infection prevention and control programs are almost exclusively staffed by infection preventionists, the overwhelming majority of whom do not prescribe or manage antibiotics. Antibiotic stewardship programs must be staffed by people who are very familiar with antibiotics. Also, though both groups share some common goals of reducing antibiotic resistance and $C$. difficile, the types of interventions the two programs seek to implement are also fundamentally different. Finally, the ultimate goals of both programs are different; infection prevention and control programs seek to eliminate healthcare-associated infections, while antibiotic stewardship programs seek to ensure that all patients get optimal antibiotic therapy.

Comment: One commenter stated that, given the size and overall staff of freestanding IRFs and LTCHs, some facilities may need additional time to incorporate these new ASP staffing requirements.

Response: We agree that these new provisions might require additional time to implement beyond the standard 60 days for all facilities, not just IRFs and LTCHs. Therefore, as discussed above, the provisions regarding antibiotic stewardship will become effective and be enforced 6 months after the effective date of this final rule for all facilities. IRFs and LTCHs are still required to comply with the hospital CoPs, so changes to the hospital CoPs also apply to IRFs and LTCHs.

Comment: One commenter appreciated the flexibility afforded in the requirements regarding the leader of the ASP, but believes there is value in that position being further defined, and recommend that the ASP professional requirements be clarified and explicitly state the person must hold either a formal M.D. or Pharm. D. degree in order to comply with the regulation.

Response: While this most likely will be the case in practice, we believe that the requirements should remain flexible for hospitals and CAHs to make these determinations for themselves.

Therefore, we believe that the hospital leadership should determine the appropriate qualifications for the leader of the ASP. However, we note here that the CDC Core Elements of Hospital Antibiotic Stewardship Programs (https://www.cdc.gov/antibiotic-use) healthcare/implementation/coreelements.html\#lead) recommend including both a physician and a pharmacist (especially those with formal training and experience in infectious diseases and/or antibiotic stewardship) to co-lead the hospital AS program and to be accountable for it. We urge hospitals and CAHs to consider these recommendations when they set their ASP leadership qualifications and when hiring the appropriate staff to develop and lead these programs.

Comment: One commenter suggested that in smaller facilities CMS should give some consideration to flexibility in staffing if the goals of the program are met and a single person is capable of handling both roles and ensuring that both priorities are met.

Response: The leaders of the infection prevention and control and the antibiotic stewardship programs must have the training required to do those jobs effectively. While there are specific types of knowledge required to lead each program (that is, knowledge about infection prevention and control best practices and knowledge about antibiotic prescribing and antimicrobial resistance), there is nothing in the regulatory language that would preclude a properly trained person from leading both programs.

Comment: Several commenters urged us to be flexible in the implementation of these provisions for all hospitals, but 
especially for smaller hospitals and CAHs, due to the time and effort it will take to fill leadership positions and develop their programs.

Response: We appreciate this comment and agree. We also agree that some smaller hospitals and CAHs may need extra technical assistance to implement the new provisions in a way that truly improves patient care. We are committed to partnering with federal and other partners to provide that assistance. For example, the CDC initiated an effort with The American Hospital Association, the Federal Office of Rural Health Policy, and the Pew Charitable Trusts to work with several CAHs that have successful antibiotic stewardship programs to learn best practices and implementation suggestions that can be shared with other critical access hospitals. The Implementation of Antibiotic Stewardship Core Elements at Small and Critical Access Hospitals and related tools released in 2017 provides guidance on practical strategies to implement antibiotic stewardship programs in small and critical access hospitals (https://www.cdc.gov/ antibiotic-use/healthcare/ implementation/core-elements-smallcritical.html).

Comment: One commenter did not support our proposal to require that the leaders of the infection prevention and control and antibiotic stewardship programs be specifically appointed by the governing body of a hospital or CAH.

Response: We appreciate this concern. The goal of this proposed requirement was to ensure that the infection prevention and antibiotic stewardship leaders are vested with authority from the leadership of the hospital or CAH. To maintain this concept while allowing more flexibility, we have changed these requirements for hospitals and CAHs. Specifically, we have revised sections $\S \S 482.42(a)(1)$ and 485.640(a)(1) of the final rule to provide that the hospital (or $\mathrm{CAH}$ ) must ensure that an individual (or individuals), who are qualified through education, training, experience, or certification in infection prevention and control, are appointed as the infection preventionist(s)/infection control professional(s) responsible for the infection prevention and control program. The selection process must include meaningful opportunity for input from members of the medical and nursing staffs and leadership.

We have also revised $\S \S 482.42(\mathrm{~b})(1)$ and $485.640(\mathrm{~b})(1)$ to now provide that the hospital (or CAH) ensure that an individual (or individuals), who is qualified through education, training, or experience in infectious diseases and/or antibiotic stewardship, is appointed as the leader(s) of the antibiotic stewardship program. The selection process must include meaningful opportunity for input from members of the medical, nursing, and pharmacy staffs.

Comment: One commenter urged CMS to modify the proposed standards regarding the demonstration of improvements in antibiotic stewardship. The commenter does not believe it is appropriate or accurate to solely use antibiotic resistance within the hospital to demonstrate antibiotic stewardship program success or evaluate a hospital's antibiotic stewardship efforts. The commenter states that numerous external factors contribute to resistance patterns, including prescribing patterns of local practitioners who may not be connected to the hospital, communityonset infections, and patient transfers from other facilities. The commenter further noted that it can be difficult to demonstrate meaningful improvement over a short period of time.

Response: We appreciate the suggestion and have modified and also deleted elements of this language for both hospitals and CAHs at

$\S \S 482.42(\mathrm{~b})(2)(\mathrm{iii})$ and 485.640(b)(2)(iii), respectively, to require that hospital and CAH ASPs must "document any" improvements, including sustained improvements, in proper antibiotic use. We agree that it would not be appropriate to use antibiotic resistance within the hospital (or CAH) as the sole criterion to demonstrate antibiotic stewardship program success or to evaluate a hospital's (or CAH's) antibiotic stewardship efforts. Therefore, we have deleted this portion of the regulatory language at $\S \S 482.42(\mathrm{~b})(2)(\mathrm{iii})$ and 485.640(b)(2)(iii). We believe that this will provide hospitals and CAHs the ability to record any category of improvement in proper antibiotic use and will not restrict ASPs to demonstrating specific types of improvements like decreased antibiotic resistance (though ASPs are still encouraged to document any improvements in this area). These revisions will also implicitly acknowledge that there often external factors, as noted by the commenter, that can negatively contribute to antibiotic resistance in the facility and that can also negatively impact meaningful improvements in this area in the short term. Hospitals and CAHs will still need to ensure that their ASPs are following nationally recognized guidelines and best practices while documenting the evidence-based use of antibiotics.
Comment: One commenter recommended that in order to clarify the organization of the antibiotic stewardship and infection prevention and control programs, the following change be made to the existing language in the preamble of the proposed rule:

"Antibiotic Stewardship, as a component of controlling infection, has long been recognized as one of the special challenges that hospitals must meet in order to address the problems of multidrug-resistant organisms and Clostridium difficile infections (CDIs) in hospitals and outpatient settings."

Response: We appreciate this commenter's recognition of the importance of the antibiotic stewardship and infection prevention and control programs.

Final Decision: After consideration of the comments we received on the proposed rule, we are finalizing $\S 482.42$ with some minor modifications to the overall regulatory language and with the following more substantive

modifications:

- Revise and finalize the language of $\S \S 482.42(\mathrm{a})(1)$ and 485.640(a)(1) to now require: "An individual (or individuals), who is qualified through education, training, experience, or certification in infection prevention and control, is appointed as the infection preventionist(s)/infection control professional(s) responsible for the infection prevention and control program. The selection process must include meaningful opportunity for input from members of the medical and nursing staffs."

- Revise and finalize the language of $\S \S 482.42(\mathrm{~b})(1)$ and 485.640(b)(1) to now require: "An individual (or individuals), who is qualified through education, training, or experience in infectious diseases and/or antibiotic stewardship, is appointed as the leader(s) of the antibiotic stewardship program. The selection process must include meaningful opportunity for input from members of the medical, nursing, and pharmacy staffs."

- Revise and finalize the language at $\S \S 482.42$ (b)(2)(iii) and 485.640(b)(2)(iii) to now require: "Documents any improvements, including sustained improvements, in proper antibiotic use."

\section{Technical Corrections}

Technical Amendments to § 482.27(b)(7)(ii) and § 482.27(b)(11)

In the final rule "Medicare and Medicaid Programs; Hospital Conditions of Participation: Laboratory Services," amending 42 CFR 482.27 (72 FR 48562, 48573, Aug. 24, 2007), we stated that 
HCV notification requirements for donors tested before February 20, 2008, would expire on August 24, 2015, in accordance with 21 CFR 610.48. Since the notification requirement period has expired, we proposed to remove $\S 482.27(\mathrm{~b})(11)$, “Applicability” and the corresponding requirements set out at $\S 482.27(\mathrm{~b})(7)$ (ii).

\section{Corrected Reference in $\S 482.58$}

In our review of the Hospital Conditions of Participation, we found an incorrect cross-reference at $\S 482.58(\mathrm{~b})(6)$, which currently reads "Discharge planning (§ 483.20(e))". Subsection 483.20(e) addresses coordination of the preadmission screening and resident review program, not discharge planning. SNF requirements for discharge plans are set out at $\S 483.20(1)$. Therefore, we proposed to correct the reference to read “Discharge summary (§ 483.20(l))”.

Removal of Inappropriate References to $\S 482.12(\mathrm{c})(1)$

Upon our review of the Hospital CoPs for the proposed rule, we discovered that there were several provisions that incorrectly reference $\S 482.12$ (c)(1), which lists the types of physicians and applies only to patients who are Medicare beneficiaries. Section 482.12(c) states that the governing body of the hospital must ensure that every Medicare patient is under the care of one of the following practitioners:

- A doctor of medicine or osteopathy;

- A doctor of dental surgery or dental medicine who is legally authorized to practice dentistry by the State and who is acting within the scope of his or her license;

- A doctor of podiatric medicine, but only with respect to functions which he or she is legally authorized by the State to perform;

- A doctor of optometry who is legally authorized to practice optometry by the State in which he or she practices;

- A chiropractor who is licensed by the State or legally authorized to perform the services of a chiropractor, but only with respect to treatment by means of manual manipulation of the spine to correct a subluxation demonstrated by x-ray to exist; and

- A clinical psychologist as defined in $\S 410.71$ of this chapter, but only with respect to clinical psychologist services as defined in $\S 410.71$ of this chapter and only to the extent permitted by State law.

The reference of this "Medicare beneficiary-only" requirement in certain other provisions of the hospital CoPs (which we have listed below) inappropriately links it to all patients and not Medicare beneficiaries exclusively. In fact, the Act at $\S 1861(\mathrm{e})(4)$ states that "every patient with respect to whom payment may be made under this title must be under the care of a physician except that a patient receiving qualified psychologist services (as defined in subsection (ii)) may be under the care of a clinical psychologist with respect to such services to the extent permitted under State law." In accordance with that provision, we have chosen to apply $\S 482.12$ (c) to Medicare patients. With the exception of a few provisions in the CoPs such as those directly related to $\S 482.12$ (c) described here, the remainder of the CoPs apply to all patients, regardless of payment source, and not just Medicare beneficiaries. For example, the Nursing Services CoP, at $\S 482.23$ (c)(1), requires that all "drugs and biologicals must be prepared and administered in accordance with Federal and State laws, the orders of the practitioner or practitioners responsible for the patient's care as specified under $\S 482.12$ (c), and accepted standards of practice." Since the CoPs clearly allow hospitals to determine which categories of practitioners would be responsible for the care of other patients, outside the narrow Medicare beneficiary restrictions of $\S 482.12$ (c), this reference is inappropriate and unnecessarily restrictive of hospitals and their medical staffs to make these determinations based on State law and practitioner scope of practice.

In order to clarify that these provisions apply to all patients and not only Medicare beneficiaries, we proposed to delete any inappropriate references to $\S 482.12$ (c). Therefore, we proposed to delete references to $\S 482.12$ (c) found in the following provisions: §§ 482.13(e)(5), 482.13(e)(8)(ii), 482.13(e)(14), and 482.13(g)(4)(ii) in the Patients' Rights CoP; and $\S \S 482.23(\mathrm{c})(1)$ and 482.23(c)(3) in the Nursing Services CoP. Additionally, and in the course of finalizing this rule, we discovered that we inadvertently failed to propose to delete an inappropriate reference to $\S 482.12$ (c), which is contained in the current provision at $\S 482.61$ (d) in the Special Medical Record Requirements for Psychiatric Hospitals CoP under the Special Requirements for Psychiatric Hospitals (regarding which hospital personnel may complete progress notes). Therefore, in the interests of consistency with the other revisions here, we are also deleting this reference in this final rule. We believe this to be a technical correction, for which notice and comment are unnecessary. We have noted this revision in the "Waiver of Proposed Rulemaking" discussion found above at section at I.B.14. With respect to all of these provisions, the reference to services provided under the order of a physician or other practitioner would still apply. We did not receive any comments on these proposed changes and are therefore finalizing them without change.

\section{Provisions of the Proposed}

Regulations and Responses to Public Comments for Critical Access Hospitals (42 CFR Part 485)

We identified several priority areas in the CoPs for CAHs (42 CFR part 485, subpart F) for updates and revisions. We believe that these proposed regulations would benefit the quality of care provided with a positive impact on patient satisfaction, length of stay, and, ultimately, cost per patient.

Additionally, we have proposed the following changes to the CAH CoPs, after considering the resource restrictions of remote and frontier CAHs.

\section{Organizational Structure} (§ 485.627(b))

This proposal was also included in the Medicare and Medicaid Programs; Regulatory Provisions To Promote Program Efficiency, Transparency, and Burden Reduction, Proposed Rule (83 FR 47686). We are finalizing this proposal in that final rule. We refer readers to the discussion under Section I.B.8.a for further information regarding this provision.

2. Periodic Review of Clinical Privileges and Performance $(\S 485.631(\mathrm{~d})(1)$

Through (2))

We proposed to change the current CoP at $\S 485.641$ to reflect the current QAPI format used in hospitals. As such, we proposed to retain the requirements under paragraphs $\S 485.641$ (b)(3) through (4), that are currently found under the "Periodic evaluation and quality assurance", $\mathrm{CoP}$, and relocate them under a new standard under the "Staffing and staff responsibilities" CoP at $\S 485.631$. We are not changing these requirements and believe that they are still appropriate for the $\mathrm{CAH}$ regulations. Since the current CoP under $\S 485.631$ discusses staffing requirements and responsibilities, we believe that relocating the requirement under a new standard, entitled "Periodic Review of Clinical Privileges and Performance" (§ 485.631(d)) is a more appropriate placement for the current provisions requiring a $\mathrm{CAH}$ to evaluate the quality of care provided by 
their nurse practitioners, clinical nurse specialists, certified nurse midwives, physician assistants, doctors of medicine, or doctors of osteopathy.

\section{Provision of Services}

(§ 485.635(a)(3)(vii))

We currently require CAHs at $\S 485.635$ (a)(3)(vii) to have procedures that ensure that the nutritional needs of inpatients are met in accordance with recognized dietary practices and the orders of the practitioner responsible for the care of the patients and that the requirement of $\S 483.25$ (i) of this chapter is met with respect to inpatients receiving post-hospital SNF care.

We proposed revisions to $\S 485.635(\mathrm{a})(3)(v i i)$ that would require that individual patient nutritional needs be met in accordance with recognized dietary practices and the orders of the practitioner responsible for the care of the patients, or by a qualified dietician or qualified nutrition professional as authorized by the medical staff in accordance with State law governing dietitians and nutrition professionals. In addition, we also proposed that the requirement of $\S 483.25$ (i) of this chapter is met with respect to inpatients receiving post hospital SNF care.

Comment: Commenters support CMS' efforts to allow clinicians to practice to the fullest extent of their credentials. The commenters stated that this proposed change at $\S 485.635$ requiring diets to be ordered by the practitioner responsible for the patient or a qualified dietitian or qualified nutrition professional as authorized by the medical staff and in accordance with state law, recognizes the specialized knowledge and training of dietitians and the benefit available to patients.

Response: We appreciate the comments and will finalize this change as proposed.

\section{Provision of Services (485.635(g))}

At $\S 485.635$ (g) we proposed a new requirement regarding non-

discriminatory behavior. Similar to our non-discrimination proposal for hospitals, we proposed to require that a $\mathrm{CAH}$ not discriminate on the basis of race, color, religion, national origin, sex (including gender identity), sexual orientation, age, or disability. We further proposed to require that CAHs establish and implement a written policy prohibiting discrimination on the aforementioned bases and that they inform each patient (and/or support person, where appropriate), in a language he or she can understand, of his or her right to be free from discrimination against them and how to file a complaint if they encounter discrimination. After consideration of the comments that we received, we are not finalizing our proposal and are instead deferring to the nondiscrimination requirements of Section 1557 of the Affordable Care Act. We refer readers to section III.B. 3 of this final rule for a more detailed discussion.

5. Infection Prevention and Control and Antibiotic Stewardship Programs (§ 485.640)

We proposed to remove the current requirements at $\S 485.635$ (a)(3)(vi) and $\S 485.641(\mathrm{~b})(2)$ and are adding a new infection prevention and control and antibiotic stewardship CoP at $\S 485.640$ for CAHs because the existing standards for infection control do not reflect the current nationally recognized standards of practice for the prevention and elimination of healthcare-associated infections and for the appropriate use of antibiotics.

We are finalizing the proposal that each $\mathrm{CAH}$ has facility-wide infection prevention and control and antibiotic stewardship programs that are coordinated with the CAH QAPI program, for the surveillance, prevention, and control of HAIs and other infectious diseases and for the optimization of antibiotic use through stewardship.

Comment: Commenters were supportive of the proposal to require each CAH to have facility-wide infection prevention and control and antibiotic stewardship programs that are coordinated with the CAH QAPI program. Commenters recognized that these changes will support a culture of antibiotic stewardship that will increase patient safety and quality of care.

Response: We appreciate the comments received on the proposed changes for the CAH infection control and antibiotic stewardship programs and will finalize the changes as proposed.

Comment: Commenters noted that CAHs would need time, resources, flexibility and support to adapt to the antibiotic stewardship requirements, especially given the fact that many do not have staff pharmacists available at all times.

Response: We also appreciate these comments. While we understand that CAHs may have less resources available, we encourage CAHs to utilize the existing available resources to ensure the antibiotic stewardship requirements are met. While small and critical access hospitals face special challenges in implementing antibiotic stewardship programs, antibiotic stewardship is no less important in these settings. Small and critical access hospitals also have some factors that can support improvements in care, as they are often tight-knit communities where collaboration is the norm. The CDC has resources available for training and education as well as support for providers implementing antibiotic stewardship programs specifically for CAHs. We also encourage CAHs to work with other hospitals or CAHs in their network (if available) for

pharmaceutical support. CAHs should also be encouraged to work with their respective quality improvement network(s)/organization(s) and health departments for additional support and resources. Additionally, we encourage CAHs to use the technical assistance available from their State Flex Program. CAHs can find contact information for their State Flex Program at https:// www.ruralcenter.org/tasc/flexprofile.

Final Rule Action: We are finalizing the proposed changes without revision.

§ 485.640(a)(1) Through (2) Infection Control Officer(s); and Prevention and Control of Infections Within the CAH and Between the CAH and Other Healthcare Settings

At $\S 485.640$ (a)(1) we proposed that the CAH ensure that an individual (or individuals), who are qualified through education, training, experience, or certified in infection, prevention and control, are appointed by the governing body, or responsible individual, as the infection preventionist(s)/infection control professional(s) responsible for the infection prevention and control program at the $\mathrm{CAH}$ and that the appointment is based on the recommendations of medical staff and nursing leadership.

We proposed at $\S 485.640$ (a)(2) that the infection prevention and control program, as documented in its policies and procedures, employ methods for preventing and controlling the transmission of infections within the $\mathrm{CAH}$ and between the CAH and other healthcare settings. The program, as documented in its policies and procedures, would have to employ methods for preventing and controlling the transmission of infection within the CAH setting (for example, among patients, personnel, and visitors) as well as between the CAH (including outpatient services) and other institutions and healthcare settings.

Comment: Commenters were generally supportive of the proposal for CAHs to have a qualified individual (or individuals) in the role of the infection preventionist(s)/infection control professional(s). 
Response: We appreciate the comments received on the proposed changes for this CAH proposal.

Final Rule Action: We are finalizing the proposed changes without revision.

§ 485.640(a)(3) Healthcare-Associated Infections (HAIs)

We proposed at $\S 485.640$ (a)(3) that the infection prevention and control program include surveillance, prevention, and control of HAIs, including maintaining a clean and sanitary environment to avoid sources and transmission of infection, and that the program also address any infection control issues identified by public health authorities.

Comment: Commenters were supportive of the proposal for CAHs to have an infection prevention and control program that includes surveillance, prevention, and control of HAIs.

Response: We appreciate the comments received on the proposed changes for this CAH proposal.

Final Rule Action: We are finalizing the proposed changes without revision.

$\S 485.640$ (a)(4) Scope and Complexity

We proposed at $\S 485.640$ (a)(4) that the infection prevention and control program reflects the scope and complexity of the services provided by the $\mathrm{CAH}$.

Comment: Commenters were supportive of the proposal for CAHs to have an infection and prevention and control program that reflects the scope and complexity of the services provided by the CAH, with one commenter requesting that specific language stating that CRNAs and other anesthesia professionals should be included in the development and leadership of infection prevention and control programs in hospitals and CAHs.

Response: We appreciate the comments received on the proposed changes for this CAH proposal. As noted in the preamble, as it relates to CAHs, staffing for these programs should be appropriate to the scope and complexity of the services offered at the CAH. We believe that CAHs should have the flexibility to include the individuals who are deemed appropriate by the $\mathrm{CAH}$ to be included in the development and leadership of these programs.

Final Rule Action: We are finalizing the proposed changes.

$\S 485.640(\mathrm{~b})(1)$ Leader of the Antibiotic Stewardship Program

We proposed at $\S 485.640(\mathrm{~b})(1)$ that the CAH's governing body ensure that an individual, who is qualified through education, training, or experience in infectious diseases and/or antibiotic stewardship is appointed as the leader of the antibiotic stewardship program and that the appointment is based on the recommendations of medical staff and pharmacy leadership.

Comment: Commenters were supportive of the proposal for the CAH's governing body to ensure that an individual with the appropriate experience is appointed as the leader of the antibiotic stewardship program. One commenter noted that this role will be fulfilled by a nurse who also has other related responsibilities and may not have the specialized training necessary for the infection preventionist role. The commenter encouraged CMS to ensure that cost effective and accessible education and training are available for $\mathrm{CAH}$ infection preventionists, and that ongoing technical assistance be provided. Additionally, the commenter requested infection preventionist expertise be available through shared services agreements across CAH networks or similar arrangements.

Response: We appreciate the comments received on the proposed changes for this CAH proposal. The proposal requires that the leader of the antibiotic stewardship program be qualified through education, training, or experience in infectious diseases and/or antibiotic stewardship. We encourage CAHs to utilize the infection control training available and resources that are available through the CDC (https:// www.cdc.gov/infectioncontrol/training/ index.html). We encourage CAHs to reach out to other CAHs (within their network or otherwise) to collaboratively meet their needs of ensuring that a leader of the antibiotic stewardship program is available to meet the needs of the CAH and its patients.

Final Rule Action: We are finalizing the proposed changes.

$\S 485.640$ (b)(2)(i), (ii), and (iii) Goals of the Antibiotic Stewardship Program

The proposed requirements at $\S 485.640(\mathrm{~b})(2)(\mathrm{i})$,(ii), and (iii) would ensure that the following goals for an antibiotic stewardship program are met: (i) Demonstrate coordination among all components of the $\mathrm{CAH}$ responsible for antibiotic use and resistance, including, but not limited to, the infection prevention and control program, the QAPI program, the medical staff, and nursing and pharmacy services; (ii) document the evidence-based use of antibiotics in all departments and services of the $\mathrm{CAH}$; and (iii) demonstrate improvements, including sustained improvements, in proper antibiotic use, such as through reductions in, CDI and antibiotic resistance in all departments and services of the hospital. We believe that these three components are essential for an effective program.

We did not receive any comments on this proposal.

Final Rule Action: We are finalizing the proposed changes.

$\S 485.640(\mathrm{~b})(3)$ and (4) Nationally

Recognized Guidelines; and Scope and Complexity

These provisions would require the $\mathrm{CAH}$ to ensure that the antibiotic stewardship program adheres to the nationally recognized guidelines, as well as best practices, for improving antibiotic use. The CAH's stewardship program would have to reflect the scope and complexity of services offered.

Comments for the identical hospital proposal are discussed with the hospital proposal in section II.B.4.

Final Rule Action: We are finalizing the proposed changes.

§ 485.640(c)(1), (2), and (3) Governing Body; Infection Prevention and Control Professionals'; and Antibiotic Stewardship Program Leader's Responsibilities

We proposed at $\$ 485.640$ (c)(1)(i) and (ii) requirements that the governing body or responsible individual ensure that:

- Systems are in place and operational for the tracking of all infection surveillance, prevention, and control, and antibiotic use activities in order to demonstrate the implementation, success, and sustainability of such activities; and

- All HAIs and other infectious diseases identified by the infection prevention and control program and antibiotic use issues identified by the antibiotic stewardship program are addressed in collaboration with $\mathrm{CAH}$ QAPI leadership.

At $\S 485.640$ (c)(2)(i)-(vi), we proposed that the responsibilities of the infection prevention and control professionals would include the development and implementation of facility-wide infection surveillance, prevention, and control policies and procedures that adhere to nationally recognized guidelines.

The governing body or responsible individual would be responsible for all documentation, written or electronic, of the infection prevention and control program and its surveillance, prevention, and control activities. Additionally, the infection preventionist(s)/infection control professional(s) would be responsible for: 
- Communication and collaboration with the CAH's QAPI program on infection prevention and control issues;

- Competency-based training and education of CAH personnel and staff including professional health care staff and, as applicable, personnel providing services in the CAH under agreement or arrangement, on the practical applications of infection prevention and control guidelines, policies and procedures;

- Prevention and control of HAIs, including auditing of adherence to infection prevention and control policies and procedures by $\mathrm{CAH}$ personnel; and

- Communication and collaboration with the antibiotic stewardship program.

Finally in this CoP, at $\S 485.640$ (c)(3), we proposed requirements for the leader of the antibiotic stewardship program similar to the proposed responsibilities for the CAH's designated infection preventionist(s)/infection control professional(s) at paragraph (c)(2). We believe that a CAH's antibiotic stewardship program is the most effective means for ensuring appropriate antibiotic use. We also believe that such a program would require a leader responsible and accountable for its success. Therefore, we proposed that the leader of the antibiotic stewardship program would be responsible for the development and implementation of a facility-wide antibiotic stewardship program, based on nationally recognized guidelines, to monitor and improve the use of antibiotics. We also propose that the leader of the antibiotic stewardship program would be responsible for all documentation, written or electronic, of antibiotic stewardship program activities. The leader would also be responsible for communicating and collaborating with medical and nursing staff, pharmacy leadership, and the CAH's infection prevention and control and QAPI programs, on antibiotic use issues.

Finally, we proposed that the leader would be responsible for the competency-based training and education of CAH personnel and staff, including medical staff, and, as applicable, personnel providing contracted services in the CAHs, on the practical applications of antibiotic stewardship guidelines, policies, and procedures.

We did not receive any comments on this proposal.

Final Rule Action: We are finalizing the proposed changes.
6. Quality Assessment and Performance Improvement (QAPI) Program (§ 485.641)

Since May 26, 1993 (58 FR 30630), the "Periodic evaluation and quality assurance review” CoP (§ 485.641) has not been updated to reflect current industry standards that utilize the QAPI model (§ 482.21) to assess and improve patient care.

We proposed to revise $\S 485.641$ (81 FR 39464) to establish new requirements for a QAPI program at a $\mathrm{CAH}$. This new requirement for CAHs would replace the existing reactive annual evaluation and quality assurance review requirement with a proactive approach of a QAPI program. A QAPI program that enables the CAH to review its operating systems and processes of care to identify and implement opportunities to provide high quality and safe care to its patients focusing on improving health outcomes and preventing and reducing medical errors.

We believe that much of the work and resources that are currently required under the existing periodic evaluation and quality assurance CoP would be utilized to adhere to the new QAPI requirement. We proposed to retain the requirements under paragraphs $\S 485.641$ (b)(3)-(4) regarding the evaluation of the diagnosis and treatment furnished by physicians and non-physician practitioners and relocate them to a new standard under the "Staffing and staff responsibilities" CoP at $\S 485.631$.

Comment: Commenters generally agree with requiring CAHs to have a QAPI program that is integrated with all of the departments within a CAH. Commenters also agree with encouraging CAHs to use proven quality improvement data to improve the quality and safety of care provided. One commenter asked about requiring CAHs to report externally for comparative benchmarking and performance improvement activities. A few commenters stated that we should require CAHs to make informed choices about where they focus improvement work to ensure their efforts have a greater benefit to the patients and communities served. Some commenters were concerned that we underestimated the time and effort it would take CAH's to implement a new QAPI program. Also, commenters requested an implementation date that is one year after the publication of this final rule and that we provide technical assistance to CAHs for the implementation of these requirements.

Response: We have taken into consideration the comment that we underestimated the time and effort it would take CAH's to implement these new QAPI requirements. We agree with an extended timeframe for implementation to allow CAHs additional time to prepare and ultimately comply with the requirements. Therefore, the requirements at $\S 485.641$ must be implemented by 18 months after the effective date of this final rule. We also encourage CAHs to utilize the technical assistance and services for CAHs that are available through the State Flex Programs, including the Medicare Beneficiary Quality Improvement Project (MBQIP), supported by HRSA's Federal Office of Rural Health Policy. CAHs can find contact information for their State Flex Program on this page, https://www.ruralcenter.org/tasc/ flexprofile. We do not require external reporting for comparative benchmarking and performance improvement activities as a condition of participation; however, we do require that CAHs maintain and demonstrate evidence of the effectiveness of its QAPI program.

Finally, we have re-evaluated our proposed requirements to eliminate unnecessary prescriptiveness proposed under paragraph (c)(1) through (6); paragraph (e); and paragraph (f)(2) through (3) and are withdrawing those proposed provisions. These changes to the proposal will allow each CAH the flexibility to implement its QAPI program in the most efficient manner for its unique circumstances.

We will require that the $\mathrm{CAH}$ meet the objectives of the QAPI program, but will allow the CAH to determine the best way to do so with respect to determining detailed program requirements, requirements related to distinct improvement projects, and details of data use. In accordance with the new requirements under $\S 465.641$ (e), CAHs will be required to incorporate quality indicator data, including patient care data and other relevant data, in order to achieve the goals of the QAPI program. We noted in our proposal suggesting that CAHs incorporate other relevant data, such as data submitted to or received from national quality reporting and quality performance programs, into their data collection analysis; however, we have removed the language referencing national quality reporting and quality performance program data from the regulatory text. We will expand on this and other examples of relevant data in the subregulatory guidance.

This data must be used by the $\mathrm{CAH}$ to achieve the objectives of the QAPI program, including addressing outcome indicators related to improved health 
outcomes and the prevention and reduction of medical errors, adverse events, CAH-acquired conditions, and transitions of care, including readmissions. This will ensure that the CAH's quality improvement efforts are evidenced based and focused on the needs of the population served by the $\mathrm{CAH}$ in a manner that best suits the unique characteristics of the CAH.

In addition, since the QAPI requirement will replace the annual evaluation requirement, we believe that a large portion of the cost can be utilized for the QAPI program because CAHs are conducting many of the functions required for an effective QAPI program. CAHs are currently required to carry out an annual evaluation of its total program. They are to evaluate their health care policies and the appropriateness of the services they provide. All patient care services and other services affecting patient health must be evaluated. Also, we have removed some of the prescriptive requirements under proposed 485.641(f)(2) through (3) for the QAPI program and recalculated the cost for implementation.

Final Rule Decision: We are finalizing the proposal, but eliminating the following proposed requirements: (6);

- Proposed paragraph (e); Performance improvement projects

- Revise the proposed requirement under paragraph (e) to remove the phrase, “. . . . such as data submitted to or received from national quality reporting and quality performance programs . . ." and

- Proposed paragraph (f)(2 through (f)(3); Program data collection and analysis.

\section{Technical Corrections}

We proposed to correct a typographical error in the regulations at $\S 485.645$ by correcting the word "provided" to "provide" in the lead first sentence. As proposed, the lead sentence would read as follows: "A $\mathrm{CAH}$ must meet the following requirements in order to be granted an approval from CMS to provide posthospital SNF care, as specified in $\S 409.30$ of this chapter, and to be paid for SNF-level services, in accordance with paragraph (c) of this section." As noted, we are also deleting an obsolete cross-reference to $\S 482.12$ (c) in our revision of the regulations text at $\S 482.61(\mathrm{~d})$.

\section{Requirements for Issuance of Regulations}

This final rule finalizes provisions set forth in "Regulatory Provisions to Promote Program Efficiency, Transparency, and Burden Reduction (CMS-3346-P,'” published September 20, 2018 (83 FR 47686), "Hospital and Critical Access Hospital (CAH) Changes to Promote Innovation, Flexibility, and Improvement in Patient Care (CMS3295-P)," published June 16, 2016 (81 FR 39448); and "Fire Safety

Requirements for Certain Dialysis Facilities (CMS-3334-P)," published November 4, 2016 (81 FR 76899).

Section 902 of the Medicare Prescription Drug, Improvement, and Modernization Act of 2003 (MMA) amended section 1871(a) of the Act and requires the Secretary, in consultation with the Director of the Office of Management and Budget, to establish and publish timelines for the publication of Medicare final regulations based on the previous publication of a Medicare proposed or interim final regulation. Section 902 of the MMA also states that the timelines for these regulations may vary but shall not exceed 3 years after publication of the preceding proposed or interim final regulation except under exceptional circumstances.

This final rule has been published within the 3-year time limit imposed by section 902 of the MMA for "Hospital and Critical Access Hospital (CAH) Changes to Promote Innovation, Flexibility, and Improvement in Patient Care (CMS-3295-P)," and "Fire Safety Requirements for Certain Dialysis Facilities (CMS-3334-P)," published November, 4, 2016 (81 FR 76899).

Additionally, a continuation notice for "Hospital and Critical Access Hospital (CAH) Changes to Promote Innovation, Flexibility, and Improvement in Patient Care (CMS3295-P) was published on June 11, 2019, (84 FR 27069). Therefore, the final rule is in accordance with the Congress' intent to ensure timely publication of final regulations.

\section{E. Collection of Information Requirements}

Under the Paperwork Reduction Act of 1995, we are required to provide 30day notice in the Federal Register and solicit public comment before a collection of information requirement is submitted to the Office of Management and Budget (OMB) for review and approval. In order to fairly evaluate whether an information collection should be approved by OMB, section 3506(c)(2)(A) of the Paperwork
Reduction Act of 1995 requires that we solicit comment on the following issues:

- The need for the information collection and its usefulness in carrying out the proper functions of our agency.

- The accuracy of our estimate of the information collection burden.

- The quality, utility, and clarity of the information to be collected.

- Recommendations to minimize the information collection burden on the affected public, including automated collection techniques.

We are soliciting public comment on each of these issues for the following sections of this document that contain information collection requirements (ICRs).

1. ICRs Regarding Quality Assessment and Performance Improvement (§ 482.21)

The existing QAPI CoP requires each hospital to:

- Develop, implement, maintain, and evaluate its' own QAPI program;

- Establish a QAPI program that reflects the complexity of its organization and services;

- Establish a QAPI program that involves all hospital departments and services and focuses on improving health outcomes and preventing and reducing medical errors; and

- Maintain and demonstrate evidence of its QAPI program for review by CMS.

We are finalizing a minor change to the program data requirements at $\S 482.21$ (b). Currently, we require that hospitals incorporate quality indicator data including patient care data, and other relevant data, for example, information submitted to, or received from, the hospital's Quality Improvement Organization.

We are updating this requirement to reflect and capitalize on the wealth of important quality data available to hospitals through several quality data reporting programs. Specifically, we are requiring that the hospital QAPI program must incorporate quality indicator data including patient care data, and other relevant data such as data submitted to or received from quality reporting and quality performance programs, including, but not limited to, data related to hospital readmissions and hospital-acquired conditions. Hospitals are likely to be participating in one or more existing quality reporting and quality performance programs such as the Hospital Inpatient Quality Reporting program, the Hospital Value-Based Purchasing Program, the Hospital Acquired Condition Reduction program, Hospital Compare, the Medicare and Medicaid Electronic Health Record 
Incentive Programs, the Hospital Outpatient Quality Reporting program, and the Joint Commission's Quality Check $^{\mathrm{TM}}$. Since a hospital is already collecting and reporting quality measures data for these programs, we do not believe that this change would increase the information collection burden for hospitals.

\section{ICRs Regarding Nursing Services (§ 482.23)}

We are finalizing our proposal to revise $\S 482.23(\mathrm{~b})$, which currently states "There must be supervisory and staff personnel for each department or nursing unit to ensure, when needed, the immediate availability of a registered nurse for bedside care of any patient," to delete the term "bedside," which might imply only inpatient services to some readers. The nursing service must ensure that patient needs are met by ongoing assessments of patients' needs and must provide nursing staff to meet those needs regardless of whether the patient is an inpatient or an outpatient. We are allowing a hospital to establish a policy that would specify which, if any, outpatient units would not be required to have an RN physically present as well as the alternative staffing plans that would be established under such a policy. The policy must take into account factors such as the services delivered; the acuity of patients typically served by the facility; and the established standards of practice for such services. In addition, the policy must be approved by the director of nursing and be reviewed at least once every 3 years.

TJC-accredited hospitals are already allowed this flexibility in nursing services policy. Those hospitals that use their TJC accreditation for deeming purposes are required to have "Leaders [who] provide for a sufficient number and mix of individuals to support safe, quality care, treatment, and services. (Note: The number and mix of individuals is appropriate to the scope and complexity of the services offered.)", (CAMH, Standard LD.03.06.01, EP 3). Further, TJC-accredited hospitals also require the "nurse executive, registered nurses, and other designated nursing staff [to] write: Nursing policies and procedures." (CAMH, Standard NR.02.02.01, EP 3). Therefore, we expect that TJC-accredited hospitals already have the policies and procedures that satisfy the requirements in this subsection, including medical staff approval and regular review. If there are any tasks that a TJC-accredited hospital may need to complete to satisfy the requirement for this subsection, we expect that the burden imposed would be negligible. Thus, for the approximately 3,900 TJC-accredited hospitals the development of policies and procedures that would satisfy this subsection would constitute a usual and customary business practice in accordance with 5 CFR 1320.3(b)(2).

The non TJC-accredited hospitals would need to review their current policies and procedures and update them so that they comply with the requirements in $\S 482.23$ (b). This would be a one-time burden on the hospital. We estimate that this would require a physician, a nurse, and one administrator. Including fringe benefits and overhead calculated at $100 \%$ of one's hourly wage, we estimate that physicians earn a total hourly compensation of $\$ 191$, administrators earn an average hourly compensation of $\$ 189$, and registered nurses earn an hourly compensation of \$71 (2017 BLS Wage Data by Area and Occupation at (https://www.bls.gov/oes/2017/may/ oes_nat.htm). We estimate that each person would spend three hours on this activity for a total of nine hours at a cost of $\$ 1,353$ (3 hours $\times \$ 71$ for a nurse's hourly wage +3 hours $\times \$ 189$ for an administrator's hourly wage +3 hours $\times$ $\$ 191$ for a physician's hourly wage). For all 1,193 non-TJC-accredited hospitals to comply with this requirement, we estimate a total one-time cost of approximately $\$ 1.6$ million $(1,193$ hospitals $\times \$ 1,353$ ). We estimate that review of the policies and procedures once every 3 years would take one hour for each individual included for a total cost of $\$ 538,043$ ( $(1$ hour $\times \$ 71$ for a nurse's hourly wage +1 hour $\times \$ 189$ for an administrator's hourly wage +1 hour $\times \$ 191$ for a physician's hourly wage) $\times$ 1,193 hospitals), or an annualized cost of $\$ 179,347$. The burden associated with these requirements will be captured in an existing information collection request (OMB Control No. 0938-0328).

3. ICRs Regarding Condition of Participation: Quality Assessment and Performance Improvement Program (§ 485.641)

$\S 485.641$ would require CAHs to develop, implement, and maintain an effective, ongoing, CAH-wide, datadriven QAPI program. The QAPI program must be appropriate for the complexity of the CAH's organization and the services it provides. In addition, CAHs must comply with all of the requirements set forth in $\S 485.641$ (b) through (e).

The current CAH CoPs at $\S 485.641$ require CAHs to have an effective quality assurance program to evaluate the quality and appropriateness of the diagnosis and treatment furnished in the $\mathrm{CAH}$ and the treatment outcomes. CAHs are currently required to conduct a periodic evaluation and quality assurance review (42 CFR 485.641(a)). They are required to evaluate its total program (for example, policies and procedures and services provided) annually. The evaluation must include reviewing the utilization of the $\mathrm{CAH}$ services using a representative sample of both active and closed clinical records, as well as reviewing the facility's health care policies. The purpose of the evaluation is to determine whether the utilization of services was appropriate, the established policies were followed, and if any changes are needed. The CAH's staff considers the findings of the evaluation and takes corrective action, if necessary (42 CFR 485.641(b)(5)(i)). Thus, we believe that all of the CAHs are performing the activities that are required to comply with many of the requirements in $\S 485.641$. However, we also believe that the CAHs would need to review their current quality assurance program and revise and, if needed, develop new provisions to ensure compliance with the requirements.

TJC accreditation standards for performance improvement (PI) already require that CAHs collect, compile, and analyze to monitor their performance (TJC Accreditation Standard PI.01.01.01 and PI.02.01.01). These TJC-accredited CAHs must also improve their performance on an ongoing basis (TJC Accreditation Standard PI.03.01.01). Thus, we believe that the 349 TJCaccredited CAHs are already in compliance with the new requirements in $\S 485.641$. However, each CAH would need to review their current practice to ensure that they are in compliance with all of the requirements under $\S 485.641$. Any additional tasks those CAHs would need to comply with the requirements for this section should result in a negligible burden, if any. Thus, the burden for these activities for the 349 TJC-accredited CAHs will be excluded from the burden analysis because they constitute usual and customary business practices in accordance with 5 CFR 1320.3(b)(2).

The 1,004 non TJC-accredited CAHs would need to review their current programs and then revise and develop new provisions of their programs to ensure compliance with the new requirements. We believe that the $\mathrm{CAH}$ QAPI leadership (consisting of a physician, and/or administrator, midlevel practitioner, and a nurse) would need to have at least two meetings to ensure that the current annual evaluation and quality assurance (QA) 
program is transitioned into the QAPI format. The first meeting would be to discuss the current quality assurance program and what needs to be included based on the new QAPI provision. The second meeting would be to discuss strategies to update the current policies, and then to discuss the process for incorporating those changes. We believe that these meetings would take approximately two hours each. We estimate that the physician would have a limited amount of time, approximately 1 hour to devote to the QAPI activities. Additionally, we estimate these activities would require 4 hours of an administrator's time, 4 hours of a midlevel practitioner's time, 8 hours of a nurse's time, and 2 hours of a clerical staff person's time for a total of 19 burden hours. We believe that the CAH's QAPI leadership (formerly the periodic evaluation and quality assurance leadership) would need to meet periodically to review and discuss the changes that would need to be made to their program. We also believe that a nurse would likely spend more time developing the program with the midlevel practitioner. The physician would likely review and approve the program. The clerical staff member would probably assist with the program's development and ensure that the program was disseminated to all of the necessary parties in the CAH. This burden estimate is slightly lower than what was published in the proposed rule because we re-evaluated our proposed requirements to eliminate unnecessary prescriptiveness. The finalized requirements are expected to allow more flexibility, and therefore slightly less burden. Since a CAH is currently required to evaluate its total program and evaluate the quality and appropriateness of the services

furnished, take appropriate action to address deficiencies and document such activities, we believe that the resources utilized on the current QA program would be utilized for the ongoing QAPI activities under $\S 485.641(\mathrm{~b})-(\mathrm{e})$. Thus, we estimate that for each $\mathrm{CAH}$ to comply with the requirements in this section it would require 19 burden hours ( 1 for a physician +4 for an administrator +4 for a mid-level practitioner +8 for a nurse +2 for a clerical staff person) at a cost of $\$ 1,657$ (\$191 for a physician $+\$ 428$ for an administrator (4 hours $\times \$ 107)+\$ 404$ for a mid-level practitioner (4 hours $\times$ $\$ 101)+\$ 568$ (8 hours $\times \$ 71$ for a nurse) $+\$ 66$ for a clerical staff person (2 hours $\times$ \$33). Therefore, for all 1,004 non TJCdeemed CAHs to comply with these requirements, it would require 19,076 burden hours $(19 \times 1,004$ non TJCdeemed CAHs) at a cost of approximately $\$ 1.7$ million $(\$ 1,657$ for each $\mathrm{CAH} \times 1,004$ non TJC-deemed CAHs). We note here the difference in hourly wage between a hospital CEO/ administrator (\$189) and a CAH CEO/ administrator (\$107). This is estimated to be an additional 15,431 hours and $\$ 1.3$ million in cost compared to the existing QA burden. The burden associated with these requirements will be captured in an existing information collection request (OMB Control No. 0938-1043).

\section{Economic Analyses}

A. Regulatory Impact Analysis for Regulatory Provisions To Promote Program Efficiency, Transparency, and Burden Reduction

\section{Statement of Need}

All major and many ostensibly minor government regulations should undergo periodic review to ensure that they do not unduly burden regulated entities or the American people, and to reflect current knowledge as to their regulatory effects. In recent years, we have revised the CoPs and CfCs to reduce the regulatory burden on providers and suppliers. In doing so, we identified obsolete and burdensome regulations that could be eliminated or reformed to improve effectiveness or reduce unnecessary reporting requirements and other costs, with a particular focus on freeing up resources that health care providers, health plans, and States could use to improve or enhance patient health and safety. We also examined policies and practices not codified in rules that could be changed or streamlined to achieve better outcomes for patients while reducing burden on providers of care, and we identified non-regulatory changes that would increase transparency and allow CMS to become a better business partner.

These final rule provisions are a continuation of our efforts to reduce regulatory burden. We are finalizing changes to the current CoPs or CfCs that will simplify and streamline the current regulations and thereby increase provider flexibility and reduce excessively burdensome regulations, while also allowing providers to focus on providing high-quality healthcare to their patients. The final rule provisions will also reduce the frequency of certain required activities and, where appropriate, revise timelines for certain requirements for providers and suppliers and remove obsolete, duplicative, or unnecessary requirements. Ultimately, these requirements balance patient safety and quality, while also providing broad regulatory relief for providers and suppliers, and reducing the associated burden on patients.

\section{Overall Impact}

We have examined the impacts of the regulatory provisions to Promote Program Efficiency, Transparency, and Burden Reduction as required by Executive Order 12866 on Regulatory Planning and Review (September 30, 1993), Executive Order 13563 on Improving Regulation and Regulatory Review (January 18, 2011), the Regulatory Flexibility Act (RFA) (September 19, 1980, Pub. L. 96-354), section 1102(b) of the Social Security Act, section 202 of the Unfunded Mandates Reform Act of 1995 (March 22, 1995; Pub. L. 104-4), Executive Order 13132 on Federalism (August 4, 1999), the Congressional Review Act (5 U.S.C. 804(2)), and Executive Order 13771 on Reducing Regulation and Controlling Regulatory Costs (January 30, 2017).

Executive Orders 12866 and 13563 direct agencies to assess all costs and benefits of available regulatory alternatives and, if regulation is necessary, to select regulatory approaches that maximize net benefits (including potential economic, environmental, public health and safety effects, distributive impacts, and equity). Section 3(f) of Executive Order 12866 defines a "significant regulatory action" as an action that is likely to result in a rule: (1) Having an annual effect on the economy of $\$ 100$ million or more in any 1 year, or adversely and materially affecting a sector of the economy, productivity, competition, jobs, the environment, public health or safety, or state, local or tribal governments or communities (also referred to as "economically significant”); (2) creating a serious inconsistency or otherwise interfering with an action taken or planned by another agency; (3) materially altering the budgetary impacts of entitlement grants, user fees, or loan programs or the rights and obligations of recipients thereof; or (4) raising novel legal or policy issues arising out of legal mandates, the President's priorities, or the principles set forth in the Executive Order.

A regulatory impact analysis (RIA) must be prepared for major rules with economically significant effects $(\$ 100$ million or more in any 1 year). We estimate that this rulemaking is "economically significant" as measured by the $\$ 100$ million threshold, and hence also a major rule under the Congressional Review Act. Accordingly, 
we have prepared an RIA that, to the best of our ability, presents the costs and benefits of these provisions of the rulemaking.

This final rule will create ongoing cost savings to providers and suppliers in many areas. Other changes we are finalizing will clarify existing policy and relieve some administrative burdens. We have identified other kinds of savings that providers and patients will realize throughout this preamble, and substantial lifesaving benefits. These life-saving effects arise by removing the incentives that were created by the current transplant center regulations to decline to transplant patients with slightly lower probabilities of success, or to decline to use organs with slightly lower probabilities of success.

We sought public comment on our burden assumptions and estimates as well as comments identifying additional reforms that should be considered for future rulemakings. As discussed later in this regulatory impact analysis, substantial uncertainty surrounds these estimates and we solicited comments on either our estimates of likely impacts or the specific regulatory changes that drive these estimates. We received, however, few comments specifically addressing our estimates. In the proposed rule, we solicited additional suggestions for things to consider that could potentially reduce burden for providers/suppliers in the future.

Comment: We received many submissions related to possible additional changes in $\mathrm{CoP} / \mathrm{CfCs}$ to reduce burden. For example, we received a number of suggestions related to additional reforms regarding the removal of barriers to the use of nurse anesthetists that could be considered for future rulemakings.

Response: Thank you for all the comments that were submitted with suggestions on how we can improve the CoPs/CfCs. Some of the suggestions are burden reducing, however some of the suggestions would be burdensome. Regardless, we will take all the suggestions in to consideration for future rulemaking.

Comment: Several commenters expressed that costs or savings attributed to QAPI, infection prevention, recertification efforts, and emergency training may have been underestimated due to the exclusion of consideration for technology changes, or other factors, in the proposed rule estimates.

Response: We thank you for your comments and recognize the uncertainty involved in our estimates. Some of our estimates have been updated to reflect new information to the extent that we are able; however, we lack the data that would be necessary to make major adjustments to many of the estimates.

Comment: One commenter inquired about what happens with all the savings being estimated for each provider or supplier.

Response: The estimated savings from reducing burden for the providers/ suppliers will allow the providers and suppliers to use those savings towards other necessary needs. We anticipate that they will have more time for patient care, and that the savings represent expenses that providers and suppliers will no longer have to incur now that we have finalized these proposals or made modifications. Some of these savings will be passed on to patients in reduced charges, but most will reduce costs charged to insurers, which will over time reduce insurance premiums to enrollees, public programs, and employer payers.

In the analysis that follows, we address the economic effects of all the major provisions of the final rule provisions. As pertinent, we indicate any significant changes from the proposed rule estimate. The analysis generally follows the typology used earlier in the preamble, and in the table that follows. As stated in the ICR section of the rule, we obtained all salary information from the May 2017 National Occupational Employment and Wage Estimates by the Bureau of Labor Statistics (BLS) at https://www.bls.gov/ oes/2017/may/oes_nat.htm and calculated the total cost per hour by adding a cost of 100 percent for overhead costs and fringe benefits.

TABLE 13-SECTION-BY-SECTION ECONOMIC IMPACT ESTIMATES

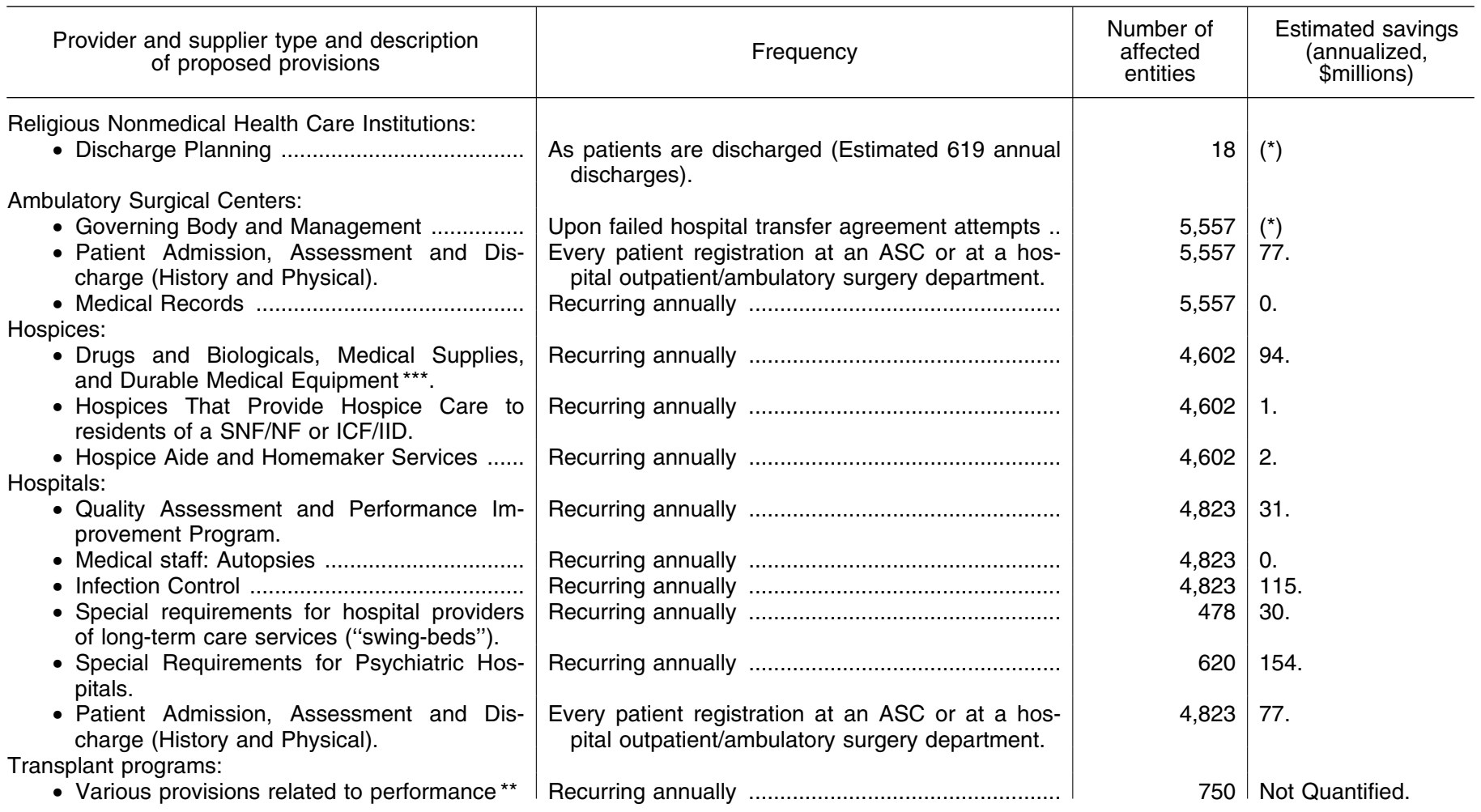


TABLE 13-SECTION-BY-SECTION ECONOMIC IMPACt EstimATES-Continued

\begin{tabular}{|c|c|c|c|}
\hline $\begin{array}{l}\text { Provider and supplier type and description } \\
\text { of proposed provisions }\end{array}$ & Frequency & $\begin{array}{l}\text { Number of } \\
\text { affected } \\
\text { entities }\end{array}$ & $\begin{array}{l}\text { Estimated savings } \\
\text { (annualized, } \\
\text { \$millions) }\end{array}$ \\
\hline 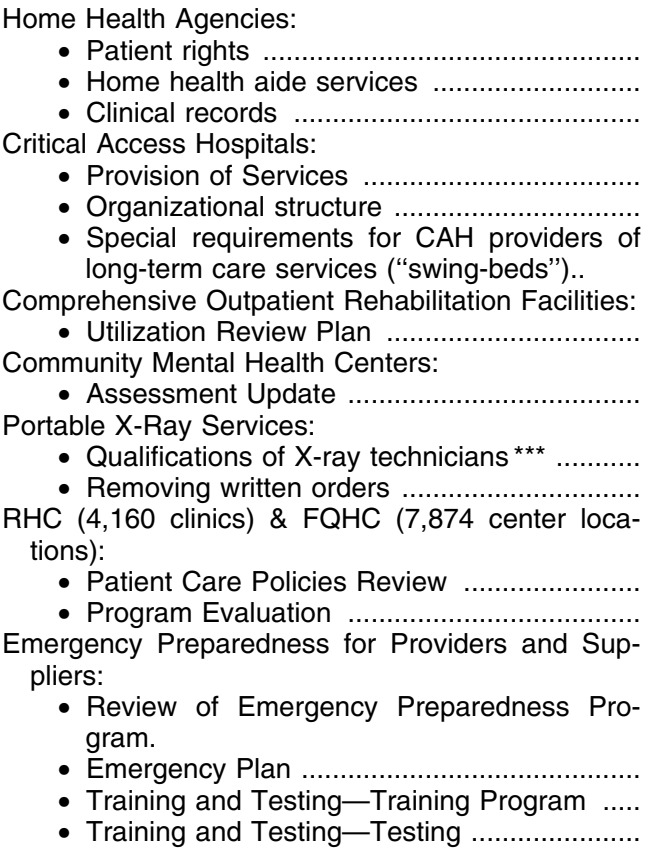 & $\begin{array}{l}\text { Recurring annually } \\
\text { Recurring annually } \\
\text { Recurring annually } \\
\text { Recurring biennially } \\
\text { Recurring annually } \\
\text { Recurring annually } \\
\text { Recurring annually } \\
\text { Recurring biennially } \\
\text { Recurring annually } \\
\text { Recurring biennially } \\
\text { Recurring annually } \\
\text { Recurring biennially } \\
\text { Recurring annually }\end{array}$ & $\begin{array}{r}12,624 \\
12,624 \\
12,624 \\
1,353 \\
1,353 \\
1,246 \\
\\
188 \\
\\
52 \\
500 \\
500 \\
\\
12,034 \\
12,034 \\
\\
56,983 \\
68,275 \\
53,543 \\
36,971\end{array}$ & $\begin{array}{l}57 . \\
\text { Not Quantified. } \\
\text { Not Quantified. } \\
1 . \\
\left(^{*}\right) \\
77 . \\
\left(^{*}\right) \\
\left(^{*}\right) \\
31 . \\
28 .\end{array}$ \\
\hline
\end{tabular}

${ }^{*}$ Amount is less than half a million dollars and rounds to zero.

${ }^{*}$ These include proposed changes to the following requirements: Special Requirements for Transplant Programs; Data submission, Clinical Experience, and Outcome Requirement for Re-approval of Transplant Programs; and Special Procedures for Approval and Re-Approval of Organ Transplant Programs.

${ }^{\star \star \star}$ This estimate is for first full year savings only and will differ in future years.

\section{Anticipated Effects}

a. Effects on Religious Nonmedical Health Care Institutions

As detailed in the Collection of Information section addressing these provisions, we reduced the discharge planning requirements for RNHCIs because RNHCIs do not provide medical treatment or services. Most patients are discharged to home or to another facility that also does not provide medical treatment or services. Although all patients must have a discharge planning evaluation, not all patients require a discharge plan. The discharge planning cost would be reduced by an estimated $\$ 22,903$.

b. Effects on Ambulatory Surgical Centers and Hospital Outpatient/ Ambulatory Surgery Departments

As of May 2017 there were 5,557 Medicare-participating ASCs. We finalized our proposal to revise the ASC CfCs in order to reduce unnecessary duplications and streamline processes in order to reduce ASC compliance burden while maintaining minimum standards for patient safety and care. The specific savings for each change are described later in this section of the rule. At $\S 416.41(\mathrm{~b})(3)$, we are removing transfer agreements and admitting privileges requirements and replacing it by mandating ASCs must periodically provide the local hospital with written notice of its operation and patient population served. This change eliminates the administrative burden associated with preparing an agreement for signature and going through the hospital credentialing process in order to obtain admitting privileges. Currently, all Medicare-certified ASCs are meeting the transfer agreement or admitting privileges requirement with the exception of approximately twenty ASCs that have tenuous relationships with their local hospital. We estimate the ASCs that do have difficulty with meeting this requirement would appreciate the annual burden savings of 2 to 4 administrator hours spent on paperwork and documentation. For those ASCs that already have transfer agreements with their local hospitals, the administrative burden is removed since transfer agreements and admitting privileges are eliminated, however, administrative burden is then replaced by the preparation and completion of the notice of operation requirement. For this reason, we have not assigned any additional burden created by the notice to the local hospital requirement. We estimate the savings at less than $\$ 10,000$ overall and largely believe this change will not produce significant savings, however, it does affect twenty or more ASCs in the short term by removing the transfer agreement requirement. We welcomed any feedback related to the time and effort for those ASCs that have secured an agreement, and if we have underestimated the savings of removing this transfer agreement in the future. As previously discussed, the enactment of EMTALA and its increasingly effective enforcement over time has rendered these transfer and admitting privileges obsolete and unnecessary. To put this point in perspective, emergencies or other unforeseen adverse events can arise in any ambulatory medical or dental setting, or in home settings. Over time, "911" emergency calls and direct ambulance responses have become standard operating procedures virtually nationwide, regardless of the place in which the problem arose. Under modern procedures, emergency responders (and patients themselves) take patients to hospital emergency rooms without regard to prior agreements between particular physicians and particular 
hospitals. Indeed, the most appropriate emergency treatment setting for a particular patient may not be one involving such an agreement, even where the agreement exists. Of course, nothing prevents particular arrangements where a hospital and ASC agree that this is beneficial for a particular type of surgery or patient condition and where patient transport can be appropriately arranged to reflect this. Accordingly, we estimate that there will be no consequential adverse health effects of this change, and therefore estimate no medical costs.

There will be competitive benefits in those places where an ASC will now be allowed to operate and provide care at reduced cost compared to inpatient treatment. Nonetheless, we believe that the number of affected areas and facilities are few, and that annual benefits are unlikely to reach the million dollar range. We sought comments on all these effects and on the preceding analysis of health effects and the majority of those we received agreed with our proposed reform.

At $\$ 416.52$ we are replacing the requirement that every patient must have a comprehensive H\&P within 30 days prior to surgery in an ASC, with a requirement that allows the operating physician and ASC to determine which patients would require more extensive testing and assessment prior to surgery. We believe that this change reduces patient and provider burden in a multitude of ways that includes the community-based physician, the ASC, and the patient. We believe that in almost all situations ASCs can reasonably rely on existing $\mathrm{H} \& \mathrm{P}$ results that are more than 30 days old and then are updated by patient responses just prior to surgery.

For ASCs, we believe this change would reduce administrative burden by decreasing the amount of time that ASC personnel spend following up on patient visits to obtain the necessary H\&P information and that it will provide for an increase in scheduling flexibility for the facility. We believe these changes may have the effect of improving patient satisfaction and increasing positive patient referrals for the ASC.

For community-based healthcare providers, to include primary care providers, we believe this change would reduce unnecessary examinations that are required to be performed and reduce administrative paperwork burden associated with providing ASCs with the necessary H\&P documentation and additional testing requirements. This change may potentially provide an opportunity for increased access to community-based providers because of available appointments that are not being filled by unnecessary patient appointments for H\&P requirements for surgery in an ASC. Those vacant appointments may also generate more revenue.
For patients, we believe this change reduces the time spent to prepare for surgery (time in community-based physician office, travel time and costs, time missed from the work place and lost productivity) and the cost associated with co-pays and other healthcare cost sharing requirements.

Finally, we believe this change reduces expenses for healthcare insurers to include Medicare, Medicaid, and private healthcare insurance companies. This change would reduce costs associated with reduced pre-operative exams, laboratory testing, chest radiographs, and echocardiograms.

In the proposed rule we stated that it is difficult to estimate the savings from this change, because they depend on a number of factors previously described, and additional factors for which we do not have precise measures, such as the number of patients (both Medicare and non-Medicare) who received two or more ASC services within the 30-day window allowed for one physical examination. This is a common occurrence because, for example, patients often receive cataract surgery on one eye and then, a week later, on the other eye. Furthermore, there are an immense number of different outpatient surgical services. At present, for example, there are about 137 services that account for about 90 percent of ASC volume, and these services are highly diverse, as shown in Table 14.

TABLE 14-TWenty Most FreQUent ASC SeRVICES IN 2015

\begin{tabular}{|c|c|c|}
\hline Surgical service & Rank & $\begin{array}{l}\text { Percent of } \\
\text { volume }\end{array}$ \\
\hline Cataract surgery w/IOL insert & 1 & 18.6 \\
\hline 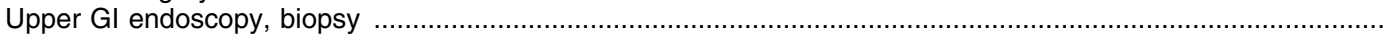 & 2 & 8.2 \\
\hline Lesion removal colonoscopy (snare technique) . & 4 & 5.6 \\
\hline Inject foramen epidural: Lumbar, sacral ............... & 7 & 4.8 \\
\hline After cataract laser surgery & 6 & 4.4 \\
\hline Diagnostic colonoscopy & 5 & 2.3 \\
\hline Colorectal screen, high-risk individual . & 10 & 2.0 \\
\hline Colorectal screen, not high-risk individual & 12 & 1.9 \\
\hline Cataract surgery, complex ........................... & 11 & 1.6 \\
\hline Injection procedure for sacroiliac joint, anesthetic & 19 & 1.3 \\
\hline 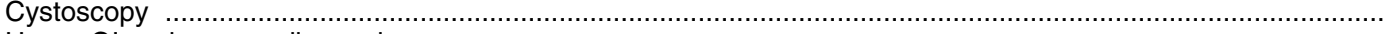 & 15 & 1.2 \\
\hline Upper Gl endoscopy, diagnosis & 13 & 1.0 \\
\hline Total & & 70.4 \\
\hline
\end{tabular}

Source: MEDPAC. Ambulatory surgical center services. 2017, p. 140.

In total, ASCs provided about 6.4 million services in 2015 (MEDPAC.
Ambulatory surgical centers services, 2017, p. 139). If we assume that 25 percent of these patients had two or more services within the 30-day 
"window" allowed in the current rule, then another H\&P with its associated battery of tests were required for each of the remaining 4.8 million individuals. Assuming that 5 percent of these patients would otherwise have already had an overall H\&P and associated tests within 30 days of the surgery, 4.56 million persons would then require a new H\&P and tests before surgery under the current requirements. In the great majority of cases involving eye or eyelid surgery of one kind or another, the ophthalmology examination preceding the ASC surgery would not have involved a comprehensive H\&P or battery of tests, and a similar situation would be involved for most other surgeries preceded by specialist rather than primary care visits.

Although we are unable to estimate the likely number of cases, one way to estimate the costs of these examinations and tests would be as follows. First, the $\mathrm{H} \& \mathrm{P}$ itself would cost approximately $\$ 100$ (the exact amount depending on diagnostic details, and not necessarily corresponding to any particular payment schedule). The battery of tests would cost approximately $\$ 100$, assuming both urine and blood testing, and, in some cases, an

electrocardiogram, but only half of physical examinations (for example, few or no ophthalmologist exams) would include such tests. The travel of the patient to and from the physician office to obtain the examination and tests would on average require 1 hour, which when valued at the average wage rate in the economy of $\$ 24$ (increased by 50 percent to include fringe benefits but not overhead) would cost about $\$ 36$. In addition, ASCs incur substantial costs for the time and trouble needed to contact physician offices and arrange for the results to be delivered. The physician offices themselves would be put through the trouble of transferring those medical records. Assuming average time spent (the median would be less but a small number of difficult cases would bring the average well above the median) would reach 10 minutes, and the use of a general office clerk at \$33 an hour, the cost per patient would average $\$ 5.50$ per patient. A further cost arises because in many cases the examination and test results simply cannot be obtained timely, and a scheduled surgery has to be postponed. Assuming that in such cases a half hour of surgeon time (at $\$ 242$ an hour) and a half hour of registered nurse $(\mathrm{RN})$ time (at $\$ 71$ an hour) is wasted, and that clerical time (\$33 an hour) to reschedule averages 10 minutes, the average cost per postponement would be $\$ 162$. (In some of these cases patient time would be wasted, as well as the time of family members accompanying the patient-we have not estimated these costs.)

Aggregating these calculations, one estimate of the annual costs of the current regulatory requirement, as shown in Table 15, could be as much as $\$ 908$ million for ASCs and a similar amount for hospital outpatient surgery. For many and perhaps most cases, however, either the surgeon or the facility would decide that $\mathrm{H} \& \mathrm{P}$ information is needed for particular patients or particular procedures, whether or not this regulatory requirement existed. Of course, it is unlikely that in such cases a strict 30day window would be insisted on. Assuming that such examination and testing information would continue to be needed for 10 percent of all patients, and that in half of these cases the information would require a new examination and tests within a 30-day window, the net costs of the regulatory reform we proposed would be 95 percent less than the preceding calculations.

As support for the proposed rule's 50 percent upper bound, the proposed rule preamble (83 FR 47733) noted that Chen et al. found that approximately 53 percent of Medicare cataract patients undergo pre-operative testing, none of which is mandated by CMS regulation. ${ }^{2}$ If these patients' physicians are cautious enough to currently pursue more preoperative activity (for example, testing and H\&P) than what is required, or state or hospital rules are driving physician behavior beyond what Medicare necessitates, then this study might be interpreted to suggest that there is little reason to believe that that behavior will change with the finalization of this rule. This study did not, however, address the 30-day time frame. We are unaware of any study or body of opinion suggesting that 30 days or any such arbitrary time limit can be medically justified, or that any providers would adhere to such a limit if not a regulatory requirement. The same points apply to other procedures performed in outpatient settings, even those such as hernia and plastic surgeries. In order to more successfully tailor the upper bound of potential cost savings to H\&P activity-rather than just extrapolating from testing behavior-we requested comment on the possibility of

\footnotetext{
${ }^{2}$ Chen CL, Lin GA, Bardach NS, Clay TH, Boscardin WJ, Gelb AW, Maze M, Gropper MA and Dudley RA, Preoperative Medical Testing in Medicare Patients Undergoing Cataract Surgery. New England Journal of Medicine 372:1530-1538, April 16, 2015.
}

building on Chen et al.’s data and methodology to estimate the increased frequency of within-30-day office visits (presumed to be H\&P) when ophthalmologist visits are at least 31 days prior to surgery relative to when ophthalmologist visits are no more than 30 days prior. We received no comments supporting (or opposing) such an estimating procedure. Regardless, laboratory testing and physical examinations have no particular dependence on each other in terms of time or place. A physician, for example, can order a laboratory test for a patient without a physical examination at all, relying on a one or two year old examination or other information. Hence, the literature on the necessity of testing is not directly germane to the question of whether a routine physical examination should occur, with or without routine blood and urine tests. To take a common example, it is universal practice for highly detailed eye tests to be performed in the surgeon's office, a week or so before cataract surgery. It is that testing on that highly specialized equipment, not a recent physical examination or blood tests ordered by a general practitioner, that determines whether, how, and with what techniques and lens inserts the cataract surgery will be performed.

As noted in the medical literature previously discussed, Chung F, Yuan H, Yin L, Vairavanathan S, and Wong DT. Elimination of preoperative testing in ambulatory surgery. Anesth Analg. 2009 Feb, 108(s):467-75, there are no known consequential medical benefits from the testing often performed in association with the current regulatory requirements for general physical examinations. This study covered hernia patients but similar results have been found in studies of cataract surgery. Accordingly, eliminating the testing that occurs during or after $\mathrm{H} \& \mathrm{P}$ could in theory produce very substantial annual ASC cost savings with no offsetting medical cost increases or harm to patients. H\&P itself, however, is distinct from testing, and literature indicating that testing is wasteful does not necessarily speak to the importance of H\&P. There are, however, no known studies supporting the proposition that $\mathrm{H} \& \mathrm{P}$ procedures should be performed within 30 days of surgery to avoid adverse consequences to patients. We received no public comments making such a claim and the great majority of those addressing this issue recommended removing at least the 30day rule, and usually the entire requirement. 
In addition, Schein et al. and Bass et al. suggest that regulations play a prominent role in the persistence of low-value H\&Ps and testing. They note that prior research indicates that it may often be the case that each member of a care team individually believes there is little value in preoperative testing for certain procedures, but those same individual physicians may fear that one or more of the other specialists or the institution may require certain tests. ${ }^{3} 4$ Therefore, the requirement for a preoperative H\&P, especially within 30 days of a surgery, greatly increases the likelihood for miscommunication among the care team regarding what tests may or may not be required. It follows that the persistence of low-value testing may simply be due to our requirement for what are often lowvalue H\&Ps, as opposed to an indication that care teams are consciously pursuing preoperative care beyond what Medicare requires, or that they would continue to do so in the absence of such a requirement.

As discussed in "Provisions of the Proposed Regulations," section II.D. 2. of the proposed rule, there is a similar regulatory requirement for hospital outpatient surgery. Based on the substantial similarity between these two service settings, we also proposed to eliminate these requirements for such surgery. Although we do not have detailed data for hospital outpatient surgery, it is widely agree to be roughly equal in size and composition to ASC surgery, though spending is higher because a higher payment schedule is used by some insurers, including Medicare, for most hospital outpatient surgery. Regardless, estimates should be based on economic costs, not any particular payment schedules. Accordingly, potential total annual savings, and hence benefits, for both settings taken together could be as much as $\$ 1.7$ billion or more. This would depend on whether hospital-based outpatient surgery decisions parallel those of independent ASCs.

If, after ASCs and hospitals make policy decisions on which types of outpatient/ambulatory surgery patients would continue to require a comprehensive H\&P, and only 50 percent of current costs were continued, potential total annual savings, and hence benefits, for both settings taken together would be about $\$ 908$ million, assuming that hospital-based outpatient surgery H\&P policy decisions parallel those of independent ASCs.

Alternatively, if 75 percent of current costs were continued, potential savings would be about $\$ 454$ million annually. While the literature shows that we can be reasonably certain that for some procedures, such as cataract surgery, few or possibly even no costs would be self-imposed, there may be other procedures where ensuing policy decisions would retain all current history and physical requirements other than the strict 30-day rule. Because of the new requirements, and other uncertainties, the potential savings from lifting the current requirements encompass at least this broad range and quite possibly more. Because there was great uncertainty in these estimates as to future decisions by ASCs and hospital outpatient departments, we decided not to present a predetermined figure in the proposed rule. Instead, we requested public comments on all the parameters of our estimates to inform the estimates we would make in the final rule. We welcomed information on likely decisions in both ASC and hospital outpatient settings, and if possible for the most common procedures shown in Table 14 and for the likelihood and cost saving effects for procedure and patient categories where the facility chooses to retain an external H\&P requirement, but extends the time window to a year or some other period that is far longer than 30 days. We did not receive any public comments on the dollar estimates but did receive a large number of public comments stating that the current H\&P requirements in their entirety and/or the 30-day limit did not rest on any medical evidence of benefits to patients, and should be removed. Even those few comments supporting retention provided no medical evidence as to the necessity of applying either an H\&P requirement or a 30 -day requirement to most outpatient surgeries.

Table 15-Current Costs and Potential Annual Savings From Creating and Obtaining Examination and Test RESULTS

\begin{tabular}{|c|c|c|c|c|c|c|c|}
\hline Type of cost & $\begin{array}{l}\text { Unit } \\
\text { cost }\end{array}$ & $\underset{\text { (M) }}{\text { Number }}$ & $\begin{array}{l}\text { Current } \\
\text { total cost } \\
(\$ M)\end{array}$ & $\begin{array}{l}\text { Twenty- } \\
\text { five } \\
\text { percent } \\
\text { retained } \\
(\$ M)\end{array}$ & $\begin{array}{l}\text { Fifty } \\
\text { percent } \\
\text { retained } \\
(\$ M)\end{array}$ & $\begin{array}{l}\text { Seventy- } \\
\text { five } \\
\text { percent } \\
\text { retained } \\
(\$ M)\end{array}$ & $\begin{array}{l}\text { Eighty- } \\
\text { five } \\
\text { percent } \\
\text { retained } \\
(\$ M)\end{array}$ \\
\hline Physical Examinations .... & $\$ 100$ & 4.56 & $\$ 456$ & $\$ 114$ & $\$ 228$ & $\$ 342$ & $\$ 388$ \\
\hline Test Batteries ......... & 100 & 2.28 & 228 & 57 & 114 & 171 & 194 \\
\hline Patient Travel Cost & 36 & 4.56 & 164 & 41 & 82 & 123 & 140 \\
\hline 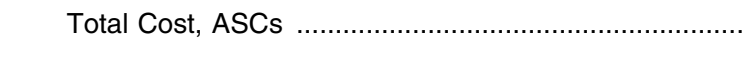 & $\ldots \ldots \ldots \ldots \ldots$ & $\ldots \ldots \ldots \ldots \ldots$ & 908 & 227 & 454 & 681 & 772 \\
\hline Total Cost, Hospital Outpatient ${ }^{* *}$ & $\ldots \ldots \ldots \ldots \ldots \ldots$ & $\ldots \ldots \ldots \ldots \ldots \ldots$ & 908 & 227 & 454 & 681 & 772 \\
\hline 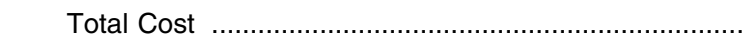 & ................... & …................ & 1,816 & 454 & 908 & 1,362 & 1,544 \\
\hline
\end{tabular}

* Based on information from a major ambulatory surgery facility, this estimate assumes that 5 percent of scheduled cataract operations are cancelled at the last minute since the required H\&P information has not arrived from the physician office where the examination was performed and the tests ordered or performed. Staff salaries must still be paid. Our estimates assume one half hour of surgeon time wasted (at $\$ 242$ an hour), one half hour of RN time wasted (at $\$ 71$ an hour), and ten minutes of clerical time (at $\$ 33$ an hour) to reschedule.

${ }^{* *}$ Hospital outpatient savings assumed to be equal to ASC savings.

${ }^{3}$ Schein OD, Pronovost PJ. A Preoperative Medical History and Physical Should Not Be a Requirement for All Cataract Patients. J Gen Intern $\overline{\text { Med. 2017;32(7) }}: 813-814$. doi:10.1007/s11606-0174043-9.

${ }^{4}$ Bass EB, Steinberg EP, Luthra R, et al. Do

Ophthalmologists, Anesthesiologists, and Internists
Agree About Preoperative Testing in Healthy Patients Undergoing Cataract Surgery? Arch Ophthalmol. 1995;113(10):1248-1256. doi:10.1001/ archopht.1995.01100100036025. 
We assume that the one-time costs of developing such policies for hospital outpatient surgery in 4,823 Medicareparticipating hospitals would be the same in the aggregate, though the mix of personnel used would be somewhat different and the cost at free-standing hospitals would likely be several times higher (for example, for involvement of the governing body and legal review). About 3,200 of these hospitals are in multi-hospital systems that would, however, reap economies of scale, and about 574 are psychiatric hospitals that we assume rarely perform surgery. In total, we estimate that, first year savings for both types of facilities would be \$38 million less, regardless of the replacement rules that each facility imposed on itself.

There are possible alternatives, including limiting the regulatory reform to the lowest risk procedures, which would probably mean almost all procedures, excluding certain procedures from the regulatory reform, exempting ASCs, but not hospital outpatient departments, changing the 30-day requirement to something much longer in duration such as 6 months or a year, and likely others. Absent contrary evidence, however, we believe that relying on physician and facility judgment maximizes benefits and presents no consequential costs.

We welcomed comments on these estimates and on both the proposal and any alternatives, and particularly welcomed any evidence-based information that would inform both our ability to provide cost savings estimates and a policy choice between either the proposed reform or an alternative. We did not receive any public comments specific to our cost estimates or recommending any alternative reform.

In the proposed rule we stated that we could not forecast with any precision what medical specialty societies, ASC governing bodies, hospital governing bodies, or accreditation bodies would decide to do in replacing the current requirement. For these reasons, we did not forecast a specific level of cost savings in the proposed rule, and simply presented a range of from $25 \%$ to $75 \%$ (and possibly even higher or lower). The comments we received from a wide range of stakeholders suggest that there might be more ASCs than we anticipated that take advantage of the new flexibility to reduce either the numbers and types of procedures for which H\&P would be required, or to expand the 30-day limitation to a greater time window, or both. Moreover, the largest organization deemed by CMS to provide standards at least equal to those of CMS, and allowed to accredit providers based on those standards, strongly endorsed replacing the current standard with one allowing procedurespecific medical judgment, as did several organizations representing professional societies or large provider organizations. There are, however, some organizations in some states and some providers that indicated they opposed any loosening of current restrictions. Our final rule would allow them to selfimpose identical restrictions, and allow all affected providers to retain current restrictions for some categories of surgery.

As noted previously, in order to more successfully tailor the upper bound of potential cost savings, we built on Chen et al.'s methodology to estimate the increased frequency of within-30-day office visits (presumed to be H\&P) when ophthalmologist visits are at least 31 days prior to surgery relative to when ophthalmologist visits are no more than 30 days prior (and thus aspects of their own medical examinations could be used to satisfy time-sensitive regulatory requirements). More specifically, we used Medicare fee-for-service claims data for procedures performed in hospitals on an outpatient basis or in ambulatory surgical centers; following Chen et al., we limited our 2017 data set to cataract surgeries performed on patients of at least age 66 and assumed office visits within 30 days prior to surgery were associated with H\&P if the provider specialty was noted as general practice, anesthesiology, cardiology, family practice, internal medicine, geriatric medicine, nurse practitioner or physician assistant. The dependent variable in our logistic regression took the value 1 if an office visit, with a specialty as listed above, had been conducted within 30 days prior to cataract surgery and 0 otherwise. The key explanatory variable took the value 1 if an ophthalmologist visit (identified if the provider specialty was noted as ophthalmology) was within 30 days prior to surgery and 0 if it was at least 31 days prior. Control variables included patient year of birth, sex and race.

Using this methodology to model the probability that the dependent variable is equal to 1, the odds ratio of the key explanatory variable is 0.654 (95 percent confidence interval: 0.633-0.676). There are, however, several limitations to this method of analysis. Most notably, identifying ophthalmology visits by the physician specialty code proved to be unreliable, and it is unclear how many ophthalmology visits may have been missed because the physician specialty field was either blank or noted as unknown. We removed all beneficiaries from our analysis who underwent a cataract surgery in 2017, yet did not have any identifiable ophthalmology visits within that same calendar year, which limited our data set substantially.

Our overall estimate is that approximately 28 percent of cataract surgeries were preceded, within 30 days, by office visits. In the vicinity of a 28-percent rate, a roughly 8- or 9percentage-point difference in rates yields an odds ratio of 0.654 . Therefore, 8.5 percent will be used in the calculation of our primary savings estimate, with an upper bound on savings of 17 percent and a lower bound of zero.

\section{c. Effects on Hospices}

As of May 2017 there are 4,602 Medicare participating hospices. We are finalizing our revisions the hospice CoPs in order to reduce unnecessary duplications and streamline processes in order to reduce hospice compliance burden while maintaining minimum standards for patient safety and care.

At $\S 418.76$ (a) we finalized our proposal to defer to State training and competency requirements, where they exist, for hospice aides. Deferring to state requirements will streamline the hiring process because hospices would not have to verify that a job candidate's qualifications meet or exceed the Federal standard in addition to verifying that the candidate meets State requirements.

According to the BLS, 408,920 aides are currently employed in "home care". The term "home care" encompasses both home health agency and hospice employers. There are 12,624 HHAs and 4,602 hospices, meaning that hospices represent 27 percent of the "home care" employer market. Thus, we conclude that hospices employ 110,408 aides (27 percent of all aide positions in "home care"). Based on an informal survey conducted by the largest hospice industry association, 76 percent of States have their own training and competency requirements, accounting for approximately 83,910 aide positions. Hospices in these states would benefit from the change because they would be permitted to rely on the completion of state mandated training and competency programs to assure that a candidate is qualified for employment, and would no longer have to take the additional step of verifying that each potential job candidate also meet the Federal requirements. We assume a 25 percent turnover rate based on discussions with industry experts, or 20,978 aide job listings per year. Based on an assumed 20 candidates that would require the qualifications verification per job 
listing, we estimate that hospices must verify the training and competency program content and format for 419,560 candidates per year. We assume that it would take 10 minutes per candidate to verify compliance with the Federal requirements, for a total of 69,927 hours per year nationwide. At a cost of $\$ 33$ per hour for a general office clerk to perform this check, we estimate that hospices will save $\$ 2,307,591$ annually.

At $§ 418.106$ (a) we are finalizing our proposal to delete the requirement that a hospice must ensure that the interdisciplinary group confers with an individual with education and training in drug management as defined in hospice policies and procedures and State law, who is an employee of or under contract with the hospice to ensure that drugs and biologicals meet each patient's needs. Not requiring the specific pharmacy advisement function will allow for more streamlined interdisciplinary group meetings. We assume that 25 percent of hospices currently use their own staff (employee or contract) for this function, and that this staff member is typically the nurse member of the interdisciplinary group. The nurse member of the interdisciplinary group is also required by $\S 418.56(a)$; therefore we believe that removing this requirement will not result in removing the expertise from the group. Rather, we believe that removing this requirement will remove the formulaic approach to interdisciplinary discussions whereby the group allots time in each meeting specifically for this discussion in order to assure regulatory compliance. In the absence of regulation, the interdisciplinary group would have the authority to decide whether the discussion is pertinent for a given patient and the information can be woven into the discussion at large. This approach has the potential to reduce the overall group discussion time, particularly for the 3 members of the interdisciplinary group that are not charged with being the pharmacology expert. Based on 1.6 million hospice patients and an assumed 3 interdisciplinary group meetings per patient, there are a total of 4,800,000 interdisciplinary group meetings per year. We assume that each interdisciplinary group meeting includes 2 minutes of time specifically related to discussing the results of the pharmacy advisement service for purposes of complying with the regulation, or 160,000 hours per year nationwide. At a cost of $\$ 307$ per hour $(\$ 203$ physician $+\$ 55$ social worker + $\$ 49$ pastoral counselor (BLS Occupation code 21-1010)), we estimate that removing this requirement would save $\$ 49,120,000$ annually. There are additional savings detailed in the Collection of Information section of $\$ 30,956,777$ annually due to removing this requirement.

Additionally, we believe that this change will reduce the specialist nursing time spent specifically on advisement services. We believe that moving away from a regulatory compliance "check box" approach would allow the specialist nurse to incorporate medication management more seamlessly into regular clinical practice. The 2008 Hospice CoP final rule (73 FR 32088) estimated a 1 hour burden per patient for expert pharmacy services (30 minute initial advisement per patient +215 minute update advisements) for a total cost of $\$ 71$ per patient for all advisement services (updated to 2017 dollars). We estimate that this change will reduce that time by 50 percent, to 30 minutes per patient, resulting in a $\$ 35.50$ per patient savings. Based on the assumption that 25 percent of hospices use their own employee to perform this function, we estimate that this reduction will occur for 400,000 patients nationwide (25 percent of 1.6 million hospice patients), for a total annual savings of $\$ 14,200,000$.

Together with the previously stated estimates, total savings would be $\$ 49,120,000+\$ 30,956,777+$ $\$ 14,200,000$ million $=\$ 94,276,777$ annually.

At $\S 418.112$ (f) we are finalizing our proposal to allow hospices and long term care facilities the additional flexibility to negotiate the format and schedule for orienting long term care facility staff regarding certain hospicespecific information. We believe that this will allow for innovation and streamlining, and reduce hospice compliance costs related to this requirement by 20 percent. For purposes of our analysis only, we assume that a typical hospice conducts 6 orientation sessions per year, and that each orientation requires 2 hours of time from a hospice nurse. At a cost of $\$ 71$ per hour, a typical hospice would spend $\$ 852$ each year to orient long term care facility staff. Assuming a 20 percent reduction in burden that can be achieved through innovation and streamlining, a typical hospice would save $\$ 170$ a year, or $\$ 782,340$ savings annually for all 4,602 hospices.

Taken together, these reforms will generate annual savings of approximately $\$ 97.4$ million (\$80.1 million for reduced interdisciplinary group meeting time $+\$ 14.2$ million for reduced specialty nursing time $+\$ 2.3$ million for streamlined hospice aide qualification requirements $+\$ 0.8$ million for streamlined facility staff orientation). We requested public comment regarding these burden estimates, and additional regulatory reforms to reduce the burden of the hospice CoPs, but did not receive any comments specific to our solicitation.

\section{d. Effects on Hospitals}

As of 2017, there were 4,823 Medicare participating hospitals. We revised the hospital CoPs in order to simplify some requirements and streamline processes in order to reduce burden associated with hospital compliance with the Medicare CoPs while maintaining minimum health and safety standards. The specific savings for each change are described below.

At $\S 482.21$, we are allowing for multihospital systems using a system governing body, as allowed under the CoPs, and that is legally responsible for two or more separately certified member hospitals, to have a unified QAPI program for the member hospitals subject to the system governing body. This will afford hospitals flexibility and the ability to gain efficiencies and achieve significant progress in quality by sharing best practices among all hospitals subject to the system governing body. This will be similar to current allowances for system governing bodies and unified medical staffs.

While there are no current

requirements that explicitly prohibit the sharing of best practices across a system, the current requirements for each hospital to have its own separate and distinct QAPI program and Infection Control program certainly have inhibited and stifled sharing of best practices and innovations among individual hospitals within a system as we point out in the preamble to the proposed rule, and which we support with our reference to the Health Research and Educational Trust, in partnership with the American Hospital Association March 2010 publication entitled, "A Guide to Achieving High Performance in Multi-Hospital Health Systems." This publication, along with positive public comments regarding unified medical staffs that we discussed in the May 2014 final rule and to which we refer in the proposed rule, clearly point to multi-hospitals more efficiently and effectively collecting,

disseminating, and sharing innovations, solutions, and best practices for patient care to each of its member hospitals through these unified patient care programs.

Approximately 3,493 of the 4,823 Medicare-participating hospitals 
participated in a hospital system in 2017 (American Hospital Association (AHA), Fast Facts 2019 (https:// www.aha.org/statistics/fast-facts-ushospitals)). According to the 2017 AHA Guide, there are 424 multi-hospital systems. The current regulatory burden for compliance with the QAPI program requirement is approximately $\$ 10,000$ annually per hospital or $\$ 48.2$ million annually for all 4,823 hospitals. If we were to allow a unified QAPI program for multi-hospital systems, this would remove 3,493 hospitals from the total 4,823 (replaced by the 424 multihospital systems) for a total of 1,754 hospitals/multi-hospital systems that would still need to comply. The new regulatory burden will be a total of approximately $\$ 17.5$ million annually $(1,754 \times \$ 10,000)$, for an annual total savings of approximately $\$ 31$ million. We welcomed comments on the quantitative and non-quantitative portions of the preceding discussion and seek any empirical evidence that would improve the accuracy and thoroughness of the relevant benefits estimation, but did not receive any comments specific to our solicitation.

We are removing the requirement for hospitals at $\S 482.22(\mathrm{~d})$, which states that a hospital's medical staff should attempt to secure autopsies in all cases of unusual deaths and of medical-legal and educational interest. Because this requirement is redundant and more detailed, specific requirements regarding medical-legal investigative autopsies are required by individual state law, we do not anticipate that hospitals would accrue additional savings from this change. The benefit to hospitals from eliminating this requirement is realized through a reduction in burden from no longer having to comply with two similar requirements of the Federal government and the State government. Hospitals would instead be required to follow the more detailed, specific regulations of the state in which they are located.

At $\S 482.42$, we are allowing for multihospital systems using a system governing body as currently allowed under the CoPs, and that is legally responsible for two or more separately certified member hospitals, to have a unified infection control program for those member hospitals subject to the system governing body. This will allow hospitals flexibility and the ability to gain efficiencies and achieve significant progress in infection prevention and control. This would also be similar to current allowances for system governing bodies and unified medical staffs. The current regulatory burden for compliance with the Infection Control program requirement is approximately $\$ 183$ million annually for all hospitals or $\$ 38,000$ per hospital. If we were to allow a unified Infection Control program for multi-hospital systems, this would remove 3,493 hospitals from the total 4,823 (replaced by the 424 multihospital systems) for a total of 1,754 hospitals/multi-hospital systems that would still need to comply. The new regulatory burden would be a total of approximately $\$ 66.7$ million annually $(1,754 \times \$ 38,000)$, for an annual total savings of approximately $\$ 116$ million, less the estimated cost of $\$ 1$ million described in the Collection of Information Requirements section, for an annual net savings of approximately $\$ 115$ million. We welcomed comments on the quantitative and non-quantitative portions of the preceding discussion and seek any empirical evidence that would improve the accuracy and thoroughness of the relevant benefits estimation.

At $\S \S 482.58(b)(1)$ and 485.645(d)(1) (cross-referenced long-term care requirement at $\S 483.10(f)(9)$ ) we are removing the requirement for hospital and CAH swing-bed providers to provide the right for patients to choose to or refuse to perform services for the facility and if they so choose, (a) document in the resident's plan of care, (b) noting whether the services are voluntary or paid and (c) provide wages for the work being performed given the location quality, and quantity of work requiring comparable skills. We discuss the economic impact for this provision in the ICR section of this rule, which is estimated to be $\$ 29.4$ million.

At § 482.58(b)(4) (and § 485.645(d)(4)) (cross-referenced long-term care requirement at $\S 483.24$ (c)), we are removing the requirement for hospital and CAH swing-bed providers to provide an ongoing activity program that is directed by a qualified therapeutic recreation specialist or an activities professional who meets certain requirements as listed at $\S 483.24$ (c)(2). We discuss the economic impact for this provision in the ICR section of this rule, which is estimated to be a savings of $\$ 73.5$ million

We are finalizing our proposal to remove the requirement at

$\S \S 482.58(\mathrm{~b})(5)$ and 485.645(d)(5) (crossreferenced long-term care requirement at $\S 483.70(p))$ for hospital and $\mathrm{CAH}$ swing-bed providers to employ a qualified social worker on a full-time basis if the facility has more than 120 beds. Given that this provision is not applicable to either provider type due to the regulatory requirements for each, it does not impose a burden upon hospitals and as such, its removal will not result in a savings of burden hours or dollars.

At $\S \S 482.58(b)(8)$ and 485.645(d)(8) (cross-referenced long-term care requirement at $\S 483.55(\mathrm{a})(1)$ ) we are removing the requirement for hospital and CAH swing-bed providers to assist in obtaining routine and 24-hour emergency dental care to its residents. We discuss the economic impact for this provision in the ICR section of this rule, which is estimated to be $\$ 2.9$ million for all hospital and CAH swing-bed providers.

At $\S 482.61(d)$, we are finalizing our proposal to allow non-physician practitioners to document progress notes in accordance with State laws and scope of practice requirements. We believe that clarification of the intent of the regulation is necessary and will result in non-physician practitioners (specifically, physician assistants, nurse practitioners, psychologists, and clinical nurse specialists) documenting in the progress notes for patients receiving services in psychiatric hospitals. We estimate that MDs/DOs currently spend approximately 30 minutes documenting progress notes in psychiatric hospitals, and that 33 percent of this time would be covered by non-physician practitioners. Of the 4,823 Medicare participating hospitals, approximately 620 (or 13 percent) are psychiatric hospitals. According to AHA, there were $36,510,207$ inpatient hospital stays in 2017, and therefore an estimated 13 percent of these stays were at psychiatric hospitals. The proposed change would result in a savings of $\$ 153.5$ million $(4,746,327$ psychiatric hospital stays $\times 2$ progress notes per stay $\times 0.5$ hours of physician/psychiatrist time $\times \$ 98$ per hourly wage difference between physicians/psychiatrists (\$198) and non-physician practitioners $(\$ 100$, the average wage between nurse practitioners and physician assistants) $\times$ 33 percent of physician time spent writing progress notes covered by nonphysician practitioners). This savings is equivalent to $\$ 247,575$ per psychiatric hospital per year.

Comment: We received a comment expressing concern over this estimate and whether the 30 minutes applies to each note, each patient per day, all patients per day, or some other measure; and that in any case, the total calculated amount of time spent on progress notes appears grossly underestimated.

Response: We thank you for your feedback and for calling this to our attention. We agree that our original estimate was low, and have revised our estimates to reflect 30 minutes spent on each note, assuming one progress note 
per week during an average length of stay of 12 days per patient.

e. Effects on Transplant Programs and Patients

We are finalizing the proposed revisions unchanged. For the convenience of current readers we are also repeating, essentially unchanged, the data and analysis that indicate that the proposed (and hence final) rule would have substantial life-extending benefits, perhaps in the billion dollar a year range, but that we are unable to provide a robust estimate of their overall magnitude.

There are approximately 750 Medicare approved transplant programs in the United States, of which 250 are kidney transplant programs. All Medicare approved transplant programs must be a part of a Medicare approved hospital, and many hospitals have several types of organ programs. Oversight of these programs occurs in two major ways: By the Organ Procurement and Transplantation Network (OPTN), which is a non-profit membership-based organization operated under a Federal contract administered by the Health Resources and Services Administration (HRSA), and by CMS under the CoPs. The current and long-term OPTN contractor is the United Network for Organ Sharing (UNOS), which performs many transplantation functions, including matching donated organs to waiting lists of patients who have failing organs, and reviewing the performance of transplant centers on a variety of criteria, including patient and organ survival. There is a third mechanism encouraging better transplant program performance, the SRTR (accessed at https://www.srtr.org). The SRTR, also operated under a HRSA contract, provides detailed data on the performance of all transplant programs, and allows the OPTN, individual transplant programs, and patients themselves to compare results on such vital metrics as patient survival rates after transplant.

For patients with most types of organ failure, a transplant is the only option for long-term survival. In the case of kidney failure, however, kidney dialysis is a viable medium-term and sometimes long-term option for most patients. On average these patients can survive a dozen or more years on dialysis; however, without a transplant, they suffer increasingly high morbidity and mortality rates. We provide Medicare coverage for such patients through the ESRD program. Under the ESRD program, patients receive dialysis treatment, usually three times a week, through machines that cleanse their blood in much the same way as healthy kidneys would do. Since its inception in 1973, more than one million patients have received treatment under this program. Kidney failure patients are unique in another way: Unlike most other organs, with the partial exception of some liver donations, it is possible for living individuals to donate "live" kidneys, whether the living donor is a relative or an unrelated altruistic donor. In the case of ESRD patients, the Medicare ESRD program serves almost all kidney failure patients, regardless of age, and these patients receive costly dialysis for a prolonged period of time. As is the case for all CoPs, our regulations for Medicare-approved organ transplant programs have the potential to protect all patients, not just Medicare beneficiaries.

As discussed earlier in this preamble, we have long regulated transplant programs, but put in place additional CoPs in the March 2007 final rule (72 FR 15198) in an effort to increase the quality of care by specifying minimal health and safety standards. In addition, outcome metrics (1 year graft and patient survival) were included in the regulation and mirrored the OPTN outcomes metrics as calculated by the SRTR. Over time, increased emphasis on organ and patient survival rates, as key metrics of transplant performance, created incentives for transplant programs to select organs most likely to survive after transplant without rejection, and to select recipients most likely to survive after the transplant. In particular, due to the increasing patient and organ survival rates over time, the 2007 standards have become increasingly stringent over time as an artifact of the performance calculation method established in the 2007 rule, an outcome that was never intended by CMS. In addition, the 2007 rule created performance standards that focused only on organ and patient survival rates for those who received a transplant, not on survival rates of patients awaiting transplant. We refer readers to a discussion of this problem in the following CMS compliance Guidelines that could only partially lighten this unintended regulatory burden at https:// www.cms.gov/Medicare/ProviderEnrollment-and-Certification/ SurveyCertificationGenInfo/Downloads/ Survey-and-Cert-Letter-16-24.pdf.

There is extensive literature on these incentives and other phenomena in transplant medicine that strongly suggests some unintended consequences on organ utilization (decreased use of "marginal" organs in their patients) and de-selection of some patients who are slightly less likely to survive for an extended period post-transplant. These unintended consequences have been anecdotal and measuring the extent to which they have occurred is difficult. In addition to the studies previously cited in the preamble (Adler et al., Schold et al., Dolgin et al., Stewart et al., Husain et al.), other studies on this issue include Kasiske B, Salkowski N, Wey A, Israni A, and Snyder J, "Potential Implications of Recent and Proposed Changes in the Regulatory Oversight of Solid Organ Transplantation in the United States," American Journal of Transplantation, Volume 16, Issue 12, December 2016, pages 3371-3377; Howard R, Cornell D, and Schold J, "CMS Oversight, OPOs and transplant centers and the law of unintended consequences, Clinical Transplantation, Volume 23, Issue 6, November/ December 2009, pages 778-783; and Abecassis M, Burke R, Klintmaim G, Matas A, Merion R, Millman D, Olhoff $\mathrm{K}$, and Roberts J, “American Society of Transplant Surgeons Transplant Center Outcome Requirements-A Threat to Innovation," American Journal of Transplantation, Volume 9, Issue 6, June 2009, pages 1279-1286; and Schold J, Miller C, Mitchell H, Buccine L, Flechner S, Goldfarb D, Poggio E, and Andreoni K, "Evaluation of Flagging Criteria of United States Kidney Transplant Performance: How to Best Define Outliers," Transplantation, June 2017, Volume 101, Issue 6, pages 13731380. These studies regarding the reduced number of transplants that would otherwise have occurred, yielded several relevant facts. The number of deceased donor organs that are discarded has been increasing over time and for kidneys, is above 20 percent. For example, about 33 percent of kidneys recovered from donors age 50 to 64 are discarded, as are about 62 percent of kidneys recovered from donors age 65 or older (Hart A et al., OPTN/SRTR 2015 "Annual Data Report: Kidney.", Accessed at http://

onlinelibrary.wiley.com/doi/10.1111/ ajt.14124/full). Officials of the UNOS have stated at public meetings that in their judgment up to 1,000 kidneys of the approximately 3,000 that are discarded each year are of good enough quality to be transplanted successfully. The number of organ transplantations reached record highs in $2016(33,500)$, about 20 percent more than 5 years earlier, due mainly to increased donation rates (OPTN, "United States organ transplants and deceased donors set new records in 2016." Accessed at https://optn.transplant.hrsa.gov/news/ us-organ-transplants-and-deceaseddonors-set-new-records-in-2016/). 
For purposes of this analysis, one approach to estimating effects is to isolate the number of kidneys (and other organs) that have been discarded as a result of the March 2007 rule; indeed, a reasonable assumption would be that the proposed rule's rescission of the 2007 requirements would have an equal and opposite effect. A slide presentation by UNOS researcher Darren Stewart (2017; accessed at https:// www.myast.org/sites/default/files/ ceot2017/AST

$\% 20 C E O T \% 2001 \% 20$ Stewart $\% 20$ $\% 20$ No\%20Organ $\% 20 \mathrm{Left}$

$\% 20 B e h i n d \% 20-\% 20 S 3 . p d f$, presents an estimate that about 1,110 of about 2,759 kidneys discarded in 2012 were of transplant quality and that between 500 and 1,000 of these could have been used in transplants (the most recent discard numbers, for 2016, are about 20 percent higher than in 2012 and one-third higher than in 2007). This presentation cites the study previously discussed in this preamble (Stewart et al. (2017)), that shows kidney discard rates rising from between 5 and 7 percent in the late 1980 s to 19.2 percent in 2015. Notably, the discard rate had already reached approximately 18 percent by 2007 , making the rate of increase much lower after the March 2007 rule was implemented than it had been in the previous two decades. Although this contrary evidence is far from definitive, it suggests that the effect of the March 2007 rule was too small to be observable in the kidney discard data.

Unfortunately, these and other studies have had to deal with other trends during the last two decades that greatly complicate measuring the independent effect of the 2007 rule. These include the increasing age of the donor pool and the attendant decline in some dimensions of organ quality, and the opposite effects of improved techniques for maintaining organ quality between the time of donation and the time of transplantation. As a result, the published studies using data on organ discards have had to use complicated multivariate statistical procedures in attempting to estimate the effects of the 2007 rule, and invariably conclude that their findings are subject to considerable uncertainty.

The preceding analysis focuses on discard rates as a tool that transplant programs can use to reduce risk of lower patient or organ survival rates, and hence risk of closure under the 2007 rule. A second tool that a transplant program can use to reduce its risk of lower overall patient survival rates is to remove patients who are slightly less likely to survive from its waiting list, most commonly by making a judgmental decision that the patient is "too sick for transplantation." Programs that are on the margin of receiving regulatory sanctions, or that have received such sanctions already, are particularly likely to exercise such judgments to reduce regulatory risk. Several studies have estimated specific numbers of transplant reductions due to the 2007 rule by comparing the number of patients removed from the waiting list at programs that have received regulatory sanctions to those that have not. To provide a baseline, these studies make the conservative assumption that those programs with zero sanctions have not removed any patients from their transplant waiting list in order to avoid sanctions. For kidneys, one study estimated that in the seven year period from 2007 to 2014, the lower performing programs removed from waiting lists over 2500 patients more than would have been expected absent sanctions, an average of over 350 per year (J.D. Schold et al., "Association of Candidate Removals From the Kidney Transplant Waiting List and Center Performance Oversight," American Journal of Transplantation 2016, 1276-1284). The implications, for the present time, of wait list changes initiated in 2007 is unclear. Increased mortality in 2007 among the very sick patients who were dropped from the wait list would have freed up organs for 2007's moderately sick patients; these patients otherwise would have declined in health so as to be the very sick population in 2008 . Thus the absolute level of health in 2008 would have been relatively good, in which case the phenomenon of patients being dropped from the wait list might not have perpetuated into the future, leaving little or no scope for benefits to be achieved now as a result of the proposed CoP revision. (We note that one year, from 2007 to 2008, may be an exaggeration as to the short-term nature of this wait list-related effect, but a somewhat longer tapering period could still have reached completion now, more than a decade after the implementation of the $2007 \mathrm{CoP}$, thus leaving little scope for benefits.) On the other hand, if the sickest patients in 2008 were dropped based on their relative health levels-in spite of their improved absolute health relative to the sickest patients in 2007-there would be potential wait list-related benefits from revising this $\mathrm{CoP}$ at the present time. The benefits of shifting transplants to the sickest patients from relatively less sick patients have not been quantified, but because the harm to the less sick patients would need to be netted off the benefit to the sickest patients, the per- transplant magnitude would be much lower than the per-transplant benefits of avoided organ discards.

Another quantitative study of kidney transplant effects used a similar methodology and estimated that as a result of the 2007 rule, in 2011 sanctioned programs performed 766 fewer kidney transplants than would otherwise have been the case. ${ }^{5}$ White et al.'s finding of reduced transplant volumes at particular kidney transplant centers does not necessarily indicate decreased transplant volumes overall, with the authors stating that their aggregate results "do not indicate that the introduction of the [2007] CoPs has systematically reduced opportunities for marginal candidates or that there has been a systematic shift away from utilization of higher risk deceased donor kidneys." In other words, regulatory sanctions could have triggered behavioral responses by some patients, some transplant surgeons, or some health insurance plans to shift patients away from these centers (many insurers restrict coverage through "centers of excellence" programs). Schold et al. (2013) find additional support for this phenomenon, describing their empirical result as follows: "Among 203 [adult kidney transplant] centers, 46 (23\%) were low performing (LP) . . . Among LP centers, there was a mean decline in transplant volume of 22.4 cases compared to a mean increase of 7.8 transplants among other centers." The estimated decrease per low-performing transplant center is roughly three times the increase per other center, but there are also roughly three times as many other centers as low-performing centers; as such, the most straightforward interpretation of this paper is that the same number of transplants is being concentrated in a smaller number of transplant centers. This outcome could still have real impacts, such as changes in travel time for patients, but although these impacts are valid for inclusion in a regulatory impact assessment, they would be much smaller in magnitude than the longevity benefits emphasized elsewhere in this analysis.

A feature common to most of these studies that is that they use data that are already several years old when the study is published, both because of the usual publishing lag and because performance data such as one-year survival rates necessarily make transplant program results less timely. None of these studies covers the last two

${ }^{5}$ Sarah L. White et al., "Patient Selection and Volume in the Era Surrounding Implementation of Medicare Conditions of Participation for Transplant Programs," Health Services Research, April 2015. 330-350. 
or three years of transplant program performance. As a result, none of these studies has been able to use actual data to assess the effects of the May 13, 2016 CMS changes that slightly reduced the performance level for finding a "condition-level" violation that threatens program closure. For recent reviews of potential effects of those changes see BL Kasiske et al, Potential Implications of Recent and Proposed Changes in the Regulatory Oversight of Solid Organ Transplantation in the United States,' Am J Transplant, December 2016, 16(12), 3371-3377, and Colleen Jay and Jesse Schold, Measuring transplant center performance: The goals are not controversial but the methods and consequences can be, Curr Transplant Rep, March 2017, 4(1), 5258 . Using past data to measure potential effects, these studies predict little or no positive effect from the revised standards (which both studies conclude will still mis-identify lower performing programs), but cannot evaluate actual effects because post-issuance evidence is not yet available. This may not be relevant policy-wise, since we proposed to eliminate those standards, but it is a key question for estimating the remaining scope (if any) of CoPassociated unnecessary organ discards, and it does flag the pervasive problem of timeliness of data and timeliness of study findings.

There are several studies that make similar estimates for liver transplant programs (for example, L.D. Buccini, et al., "Association Between Liver Transplant Center Performance Evaluations and Transplant Volume," American Journal of Transplantation 2014, 2097-2105). This study found a large difference in transplant volume between programs rated as lower performing by the SRTR (average decrease of 39.9 transplants from 2007 to 2012) and those not receiving adverse SRTR ratings (average increase of 9.3 transplants over the same period). The 27 lower performing centers thus reduced their total number of liver transplants by over 1,000, and compared to the higher performing centers the decrease was even larger. This study did not, however, tie its estimates to the performance standards in the 2007 rule (which are similar but not identical to SRTR standards), to sanctions under that rule, or to specific center decisions, such as removing candidates from the wait list. Hence, while it certainly contributes to the body of scholarship indicating that since 2007 transplants have been performed in a more concentrated set of programs, it does not appear to provide direct estimates of the quantitative effects of the 2007 rule on overall numbers of liver transplants.

Taking into account all the various uncertainties involved in these studies, we did not and do not believe that we can estimate the effects of the 2007 rule on numbers of transplantations for any organ other than kidneys, and that even for kidneys there is no clear central estimate of likely quantitative effects. The wide variation in published results, and the disclaimers as to the various uncertainties involved, make a precise as well as reliable estimate all but impossible and would render arbitrary any non-zero lower bound estimate of health and longevity impacts. (As noted above, however, even in the absence of health and longevity effects, there may be other benefits, such as reduced travel costs, if the proposed rule reduces concentration of transplants in a smaller number of facilities.) Therefore, we have shown the effects of the final rule change as "not quantified." This is not unusual in regulatory impact analyses that address complex phenomena that cannot be measured directly, or whose effects are intertwined with other changing circumstances.

Every transplant quality organ that is used for transplantation rather than discarded has a very high probability of substantially extending the life of the recipient. There is a particularly extensive literature on life expectancy before and after transplant, quality of life, and cost savings for kidney patients. A literature synthesis on "The Cost-Effectiveness of Renal

Transplantation," by Elbert S. Huang, Nidhi Thakur, and David O. Meltzer, in Sally Satel, When Altruism Isn't Enough (AEI Press, 2008) found essentially universal agreement that kidney transplants were not only substantially life extending, but also cost reducing. The authors performed an extensive literature search and found that from 1968 to 2007 seventeen studies assessed the cost-effectiveness of renal transplantation. The authors concluded that "Renal transplantation . . . is the most beneficial treatment option for patients with end-stage renal disease and is highly cost-effective compared to no therapy. In comparison to dialysis, renal transplantation has been found to reduce costs by nontrivial amounts while improving health both in terms of the number of years of life and the quality of those years of life" (page 31). More recent studies have reached similar conclusions, as have other syntheses. For example, the "Systematic Review: Kidney Transplantation Compared with Dialysis in Clinically Relevant Outcome" (M. Tonelli, N. Wiebe, G. Knoll, A. Bello, S. Browne, D.
Jadhov, S. Klarenbach, and J. Gill, American Journal of Transplantation 2011: 2093-2109) focused on life expectancy and quality of life. This article reviewed 110 studies, and concluded that the vast majority showed major improvement in life quality and reductions in mortality among transplant recipients compared to those remaining on dialysis. The Annual Data Report of the United States Renal Data System utilizes national data on ESRD, and reports that deaths per 1,000 patient years are about 180 for dialysis patients and about 32 for transplant recipients (see 2016 report, volume 2, Figure i.13 and Tables H.4 and H.10); accessed at https://www.usrds.org/adr.aspx). There are similar data on other organs. For example, in 1998, HHS published a final rule with comment period that established governance procedures for the OPTN (63 FR 16296). In the RIA for that rule, the Department estimated that "the annual benefits of organ transplantation include about eleven thousand lives vastly improved by kidney transplantation, and another eight thousand lives both vastly improved and prolonged by transplantation of other major organs", (63 FR 16323).

Even without a robust aggregate estimate of likely increases in organ utilization as a result of this proposed regulatory change, the potential benefits are very substantial. For each new kidney transplantation, there would be an average of 10 additional life years per transplant patient compared to those on dialysis (see Wolfe A et al.,

"Comparisons of Mortality in All

Patients on Dialysis, Patients on Dialysis Awaiting Transplantation, and

Recipients of a First Cadaveric

Transplant," NEJM, 1999, 341:1725-30; accessed at http://www.nejm.org/doi/ full/10.1056/

NEJM199912023412303\#t=article). Valuing each year of life gained using a "value of a statistical life year" (VSLY) of $\$ 490,000$ in 2014 dollars, the total benefits from each additional transplantation in 2018 would be $\$ 4.9$ million before discounting and $\$ 4.4$ million after inflating to 2016 dollars and discounting at either 3 or 7 percent over the 10-year period (life-year figure for 2014 from Office of the Assistant Secretary for Planning and Evaluation, HHS, Guidelines for Regulatory Impact Analysis, 2016, page 21, accessed at https://aspe.hhs.gov/pdf-report/ guidelines-regulatory-impact-analysis). The HHS methodology produces the same result at either discount rate in order to reach the same predetermined "real" value. For an explanation and 
justification of this VSLY approach, see Cass R. Sunstein, "Lives, Life-Years, and Willingness to Pay," 104 Columbia Law Review [i] (2004).

Those HHS guidelines also explain in some detail the concept of quality adjusted life years. The key point to understand is that these are researchbased estimates of the value that people are willing to pay for life-prolonging and life-improving health care interventions of any kind (see sections 3.2 and 3.3 of the HHS Guidelines for a detailed explanation). The QALY amount used in any estimate of overall benefits is not meant to be a precise estimate, but instead is a rough statistical measure that allows an overall estimate of benefits expressed in dollars.

An alternative and more sophisticated analysis would take into account that the life-extending effect of a kidney transplant is not its first effect, but typically follows a number of years off dialysis, until the organ fails and the patient returns to dialysis or is retransplanted. Such an analysis can be found in a recent study by P.J. Held et al., "A Cost-Benefit Analysis of Government Compensation of Kidney Donors," American Journal of Transplantation, 2016, pages 877-885 (plus 65 pages of supplementary details explaining all assumptions, data sources, and calculations). The largest differences between the base case estimated in that study and the preceding estimates is that this RIA uses the considerably higher value of a statistical year of life under HHS guidelines, and this RIA uses the full value of a statistical life year without a "quality" adjustment for the added years of life (we use QALYs only for the improved quality of life during years that would otherwise be on kidney dialysis). Under such an estimation approach, potential life-extending benefits could be somewhat larger. For example, if the proposed reform increased the number of life-extending kidney transplants by only 100 a year, and the benefits of both additional life years and QALY gains were estimated at $\$ 5.1$ million per patient, its total annual benefits for kidney patients would be approximately $\$ 510$ million a year (100 $\times \$ 5.1$ million) .

There are additional benefits from kidney transplantation. As previously discussed, kidney transplants do reduce medical costs, with "breakeven" after about 5 years and net savings of several hundred thousand dollars per patient. Other organ transplants create lesser or no medical savings because the alternative is not dialysis. Clearly, however, these kidney transplant savings are small in relation to the life- extending benefits. We have not estimated medical savings or costs for kidneys or other organs in this RIA because any such estimates would depend on the number of additional transplants that we have not estimated.

We note that life-extending estimates are averages across patients who vary widely in age, medical condition, and life expectancy, as well as type of organ failure. For example, the sickest patients typically have very low life expectancies without transplant, and hence stand to gain the most years of life from a transplant. Partly offsetting this, these same patients, on average, have slightly lower survival rates posttransplant. Organ and patient survival issues are complex and dealt with by detailed policies and procedures developed and used by the transplant community under the auspices of the OPTN. These policies are reviewed and revised frequently based on actual experience and changing technologyover time the success rate from previously marginal organs, and in older patients, have both increased substantially. For purposes of this analysis, the proper measure is the average gain across all patients who would receive transplants as a result of eliminating the 2007 rule, net of these other factors.

There could be potential offsets to these calculated and uncalculated benefits and cost reductions. However, the particular regulatory requirements we proposed to remove are unlikely to drive any further significant increases in graft and patient survival. For renal transplants, the expected 1-year graft and patient survival rates are already at 95 percent or better. Transplant program outcomes will continue to be monitored by the OPTN and programs that are not in compliance with the OPTN outcomes are referred to their Membership and Professional Standards Committee for quality improvement activities. The SRTR also publishes detailed data on transplant program performance that allows patients and their physicians to compare transplant programs and this transparency creates pressures to maintain and improve survival rates in order to attract these patients.

The current regulatory requirements for transplant centers, as discussed in section II.E “Transplant Centers” of the proposed rule, have created both positive and adverse incentives for transplant programs, with unanticipated side effects on both utilization of donated organs and the ability of the highest risk patients to obtain transplants. We expect the changes made by this final rule to provide substantial net benefits, particularly since other regulatory and informational incentives remain in place.

We requested comments on this analysis as well as information that would enable a more robust quantitative analysis of the impacts of this change and on any alternative reforms that might provide even higher benefits. We did not, however, receive comments specifically addressing these requests.

\section{f. Effects on HHAs}

As of May 2017 there are 12,624 HHAs that participate in Medicare and Medicaid. In the January 2017 HHA CoP final rule (82 FR 4504) we estimated that compliance with the requirements at $\S 484.50(a)(3)$ related to providing oral notice of all rights to each patient would impose a burden of 5 minutes per patient, or 1,330,246 hours of burden nationwide at a cost of $\$ 80,030,370$, annually. The cost estimate was based on a $\$ 63$ per hour estimate for the services of a RN as derived from the BLS Occupational Handbook, 2014-2015 edition, including a 100 percent benefit and overhead package. Adjusted to reflect more updated salary information, as described previously, we estimate that compliance with this provision would impose a $\$ 94,447,466$ burden, based on a RN earning $\$ 71$ per hour.

We proposed to revise the verbal notification requirements to limit them to those that are required by section 1891 of the Act. Limiting the amount of information that is required to be provided orally will reduce the time per patient that is required to comply with the revised requirement. For purposes of this analysis only, we assume that providing oral notice regarding financial liability only will require 2 minutes per patient, reducing burden by 60 percent. Based on this assumption, this proposed change would reduce the burden of the patient rights notification requirement by 198,148 hours $(1,330,246$ hours originally estimated $\times 0.6)$ and $\$ 56,668,480(\$ 94,447,466$ burden as updated to reflect more recent salary estimates $\times 0.6$ ).

We are also finalizing three changes that do not have a savings estimate. First, we are eliminating the requirement at $\S 484.80(\mathrm{~h})(3)$ that the HHA conduct a full competency evaluation of deficient home health aides, and replace it with a requirement to retrain the aide regarding the identified deficient skill(s) and require the aide to complete a competency evaluation related to those skills. As we stated in the January 2017 HHA CoP final rule (82 FR 4575), it is standard practice within the HHA industry to supervise home health aides, and the regulatory requirements for such 
supervision do not impose any additional burden. We are also finalizing a change to permit HHAs to use either patients or pseudo-patients when conducting home health aide competency evaluations. While this change does not have a monetary savings estimate, we believe that this additional flexibility will increase the speed for aides completing their competency evaluations, thus increasing the pool of aides eligible to provide services and reducing patient wait times for aide services.

We requested public comment regarding these burden estimates, and additional regulatory reforms to reduce the burden of the HHA CoPs, but did not receive any specific to our burden estimates. Comments regarding additional regulatory reforms to reduce the burden on HHAs are addressed earlier in the preamble.

\section{g. Effects on CAHs}

We proposed to remove the requirement at $\S 485.627(\mathrm{~b})(1)$ for $\mathrm{CAHs}$ to disclose to CMS its owners or those with a controlling interest in the $\mathrm{CAH}$ or any subcontractor in which the CAH directly or indirectly has a 5 percent or more ownership interest in accordance with 42 CFR part 420 , subpart C. We discuss the economic impact of this provision in the ICR section, which is estimated at $\$ 143,701$ in total savings for all CAHs. We also discussed the burden reduction for our proposed revision of the "patient care policies" requirements imposed on CAHs in the ICR section of this rule, which is estimated at $\$ 2.6$ million. Finally, the effects of the CAH swing-bed provisions are covered in the ICR section of the rule, and estimated to be $\$ 76.5$ million for all CAHs.

\section{h. Effects on CORFs}

We discussed the burden reduction for our proposed revision of the "utilization review plan" requirements imposed on CORFs in the ICR section of this rule, which is estimated at $\$ 315,840$.

\section{i. Effects on CMHCs}

We discussed the burden reduction for our proposed revision of $\S 485.914(\mathrm{~d})(1)$ "update of the comprehensive assessment" requirements imposed on CMHCs in the ICR section, which is an estimated savings of $\$ 156,975$.

\section{j. Effects on Portable X-Ray Services}

At $\S 486.104$ we proposed to revise the portable x-ray CfCs to focus on the qualifications of the technologist performing the diagnostic test. As of
May 2017 there were approximately 500 Medicare-participating portable x-ray suppliers employing an estimated 5,000 portable $x$-ray technologists. Hiring limited x-ray technologists or those with State licensure would allow portable $\mathrm{x}$ ray suppliers to fill vacant positions at a lower hourly cost. Assuming a 10 percent annual turnover rate, all technologists could be hired at the lower salary over a period of 10 years. Limited x-ray technologists can be hired for approximately $\$ 30$ an hour $(\$ 62,400$ per year), whereas, according to the BLS, x-ray technologists with advanced certification (ARRT) are hired at a rate of approximately $\$ 60$ dollars per hour (\$124,800 per year). This creates a savings opportunity of $\$ 30$ per hour, or $\$ 62,400$ per year, per technologist position. Based on an assumed 10 percent turnover rate, or 500 positions filled in any given year, this change would create a savings of $\$ 31,200,000$ savings in the first year. We believe that these savings would be increased every year as more positions are filled at the lower salary rate.

We discuss the economic impact for the requirements regarding written orders in the ICR section of this rule, which represents $\$ 27.7$ million in savings.

We requested public comment regarding these burden estimates, and additional regulatory reforms to reduce the burden of the portable x-ray CfCs, but did not receive any comments specific to our solicitation.

\section{k. Effects on RHCs and FQHCs}

We discussed the burden reduction for our revision of $\S 491.9(b)(4)$ "review of patient care policies" requirements imposed on RHCs and FQHCs in the ICR section, which is an estimated savings of $\$ 7.3$ million biennially, or approximately $\$ 3.7$ million annually.

In addition, the burden reduction for our revision of $\S 491.11$ (a) "program evaluation" requirements imposed on RHCs and FQHCs in the ICR section of this rule, which is an estimated savings of $\$ 9.9$ million biennially, or approximately \$5 million annually.

1. Effects of Emergency Preparedness Requirements on Providers and Suppliers

The revisions to the emergency preparedness requirements for Medicare and Medicaid participating providers and suppliers, as discussed in section II.M, either simplify the requirements, eliminate duplicative requirements, or reduce the frequency in which providers would need to comply with the emergency preparedness requirements. We estimate that the finalized changes to the emergency preparedness requirements would accrue an annual cost savings of $\$ 124$ million in total. The potential, estimated cost savings for each revised emergency preparedness requirement is outlined in detail below. The methodology used to calculate the economic impact and the costs associated with the changes to the emergency preparedness requirements is the same methodology used to calculate the economic impact in the Emergency Preparedness final rule (81 FR 63860).

At $\S 482.15(a),(b),(c)$, and (d) for hospitals and parallel regulatory citations for other facilities, we are finalizing our proposal for all providers except LTC facility providers, to review their program at least every 2 years. We discuss the economic impact for this requirement in the ICR section of this rule, which represents annualized cost savings of $\$ 69,639,324$, or approximately $\$ 139$ million biennially.

At § 482.15(a)(4) for hospitals, and other parallel citations for the facilities mentioned in section II.J.2 of the rule, we eliminated the requirement that facilities document efforts to contact local, tribal, regional, State, and Federal emergency preparedness officials and that facilities document participation in collaborative and cooperative planning efforts. We discuss the economic impact for this requirement in the ICR section of this rule, which represents $\$ 7,319,255$ in savings.

At $\S 482.15(d)(1)(i i)$ for hospitals, and other parallel citations for other

facilities mentioned in section II.J.2 of the rule, we are finalizing our proposal for all providers, except LTC facilities, to require that facilities provide training biennially, or every 2 years, after facilities conduct initial training on their emergency program. In addition, we are requiring additional training when the emergency plan is significantly updated. We discuss the economic impact for this requirement in the ICR section of this rule, which represents annualized cost savings of $\$ 25,593,781$, or approximately $\$ 51$ million biennially.

Finally, at $\S 482.15(d)(2)$, we are finalizing our proposal to require that providers of inpatient services mentioned in section II.J.2 of the rule conduct two testing exercises annually, one of which may be an exercise of their choice that must be either a communitybased full-scale exercise (if available), an individual facility-based functional exercise, a drill, a tabletop exercise or workshop that includes a group discussion led by a facilitator. We are requiring that providers of outpatient services mentioned in section II.J.2 of 
the rule conduct one testing exercise annually which must be either a community-based full-scale exercise (if available) or an individual facility-based functional exercise every other year, and in the opposite years, may be either a community-based full-scale exercise (if available), a facility-based functional exercise, a drill, or a tabletop exercise or workshop that includes a group discussion led by a facilitator. We discuss the other economic impacts for this requirement in the ICR section, which represents $\$ 9,296,422$ in savings. We do not estimate any economic impact for the providers of inpatient services as we did not propose any changes to the number of testing exercises that must be conducted by these providers; however, we estimate an additional economic impact for this provision for each outpatient provider due to a reduction in the testing requirement from two exercises per year to one exercise per year. We would like to note that for CORFs and

Organizations, consistent with the Emergency Preparedness Final Rule (Medicare and Medicaid Programs; Emergency Preparedness Requirements for Medicare and Medicaid Participating Providers and Suppliers; Final Rule, 81 FR 63860), the CoPs for these providers previously required them to have ongoing drills and exercises to test their disaster plans. Therefore, we continue to expect, as we did in the Emergency Preparedness final rule, that the economic impact to comply with this requirement will be minimal, if any. Therefore, the total economic impact of this provision for CORFs and Organizations will be limited to the estimated ICR burden of $\$ 56,212$ and $\$ 310,362$, respectively.

We estimate a total impact savings of $\$ 11,238,093$ for this change. With an estimated ICR savings of $\$ 9,296,422$, we estimate that the total economic impact of this policy for the affected providers will be $\$ 20,534,515$. We list a summary of the calculation for the impact savings accrued by removing this requirement for each facility in Table 16, based on facility numbers available as of May 2017.

- ASCs: Combined total savings of $\$ 2,000,520$ for 5,557 ASCs ( 4 hours for an administrator at $\$ 109$ per hour plus 4 hours for a registered nurse at $\$ 71$ per hour) $\times 5,557$ ASCs $\times 50$ percent).

- Outpatient Hospice: Combined total savings of $\$ 1,438,240$ ( $(4$ hours for an administrator at $\$ 107$ per hour plus 4 hours for a registered nurse at $\$ 71$ per hour) $\times 4,040$ outpatient hospices $\times 50$ percent).

- PACE: Combined total savings of $\$ 16,543$ ((1 hour home for a care coordinator at $\$ 71$ per hour plus 1 hour for a quality improvement nurse at $\$ 71$ ) $\times 233$ PACEs $\times 50$ percent).

- HHAs: Combined total savings of $\$ 2,695,224$ ( $(2$ hours for an administrator at $\$ 107$ per hour plus 3 hours for a director of training at $\$ 71$ per hour) $\times 12,624$ HHAs $\times 50$ percent).

- CMHCs: Combined total savings of $\$ 60,214$ ( $(5$ hours for an administrator at $\$ 107$ per hour plus 3 hours for a nurse at $\$ 71$ per hour) $\times 161 \mathrm{CMHCs} \times 50$ percent).

- OPOs: Combined total savings of $\$ 5,162$ ( $(1$ hour for a QAPI Director at $\$ 107$ per hour plus 1 hour for an education coordinator at $\$ 71$ per hour) $\times 58$ OPOs $\times 50$ percent).

- RHCs/FQHCs: Combined total savings of $\$ 4,284,104$ ( ( $(4$ hours for an administrator at $\$ 107$ per hour plus 4 hours for a registered nurse at $\$ 71$ per hour) $\times 4,160$ RHCs $\times 50$ percent) $\$ 1,480,960+$ (4 hours for an administrator at $\$ 107$ per hour plus 4 hours for a registered nurse at $\$ 71$ per hour) $\times 7,874$ FQHCs $\times 50$ percent) $2,803,144$.

- ESRDs: Combined total savings of $\$ 738,086$ ( $(1$ hour for an administrator at $\$ 107$ per hour plus 1 hour for a nurse manager at $\$ 107$ per hour) $\times 6,898$ dialysis facilities $\times 50$ percent).

TABle 16-Cost Savings for Emergency Preparedness Testing

\begin{tabular}{|c|c|c|}
\hline Provider/supplier & $\begin{array}{l}\text { Cost savings per } \\
\text { provider/supplier }\end{array}$ & Combined total savings \\
\hline ASCs & $\$ 360$ & $\$ 2,000,520$ for 5,557 ASCs. \\
\hline Hospices (outpatient) .... & 356 & $\$ 1,438,240$ for 4,040 outpatient hospice facilities. \\
\hline 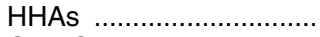 & 214 & $\$ 2,695,224$ for 12,624 HHAs. \\
\hline CMHCs $\ldots \ldots \ldots \ldots \ldots \ldots \ldots \ldots \ldots$ & 374 & $\$ 60,214$ for $161 \mathrm{CMHCs}$. \\
\hline OPOs & 89 & $\$ 5,162$ for 58 OPOs. \\
\hline ESRD Facilities & 107 & $\$ 738,086$ for 6,898 dialysis facilities. \\
\hline
\end{tabular}

\section{m. One-Time Implementation Costs}

All of the changes presented above will necessarily have to be read, and understood, and implemented by affected providers. This will create onetime costs even though the underlying change reduces burden. In most cases these costs will be very low, and may be as simple as observing that a particular procedure will need only to be performed once rather than twice a year, and changing the schedule accordingly. In some cases, the facility will need to adjust in response to multiple burden reduction changes. In still other cases, time will have to be spent deciding how to change existing policy. For example, as discussed previously, ASCs and hospital outpatient facilities will need to decide whether and in what circumstances medical histories and physical examinations will be required or encouraged as a matter of policy. Rather than attempt to estimate these situational variables in detail for each facility type, we believe it possible to make reasonable overall estimates of these one-time costs, recognizing that there will be considerable variations among provider types and among individual providers.

In total, there are about 122 thousand affected entities, as shown in the Table 17 that follows. We assume that on average there will be 1 hour of time spent by a lawyer, 2 hours of time by an administrator or health services manager, and 2 hours of time by other staff (we assume registered nurses or equivalent in wage costs) of each affected provider to understand the regulatory change(s) and make the appropriate changes in procedures. We further estimate that for one tenth of these providers, 2 hours of physician time will be needed to consider changes in facility policy. Average hourly costs for these professions, with wage rates doubled to account for fringe benefits and overhead costs, are \$136 for lawyers, \$107 for managers, \$71 for 
registered nurses, and $\$ 203$ for

physicians based on 2017 BLS data.

The estimated costs for an average provider would therefore be 1 hour at $\$ 136$ and in total for the lawyers, 2 hours at $\$ 107$ or $\$ 214$ in total for the managers, 2 hours at $\$ 71$ or $\$ 142$ in total for the other staff, and 0.2 hours at $\$ 203$ or $\$ 41$ in total for the physicians. These one-time costs add up to $\$ 533$ per provider on average, and in total to about $\$ 65$ million.

TABLE 17-ONE-TIME IMPLEMENTATION COSTS

\begin{tabular}{|c|c|}
\hline Provider type & $\begin{array}{l}\text { Number of } \\
\text { affected } \\
\text { providers }\end{array}$ \\
\hline \multicolumn{2}{|l|}{$\begin{array}{l}\text { Religious Nonmedical Health Care } \\
\text { Institutions }\end{array}$} \\
\hline \multicolumn{2}{|l|}{ Ambulatory Surgical Centers and } \\
\hline Hospices ............................. & 4,602 \\
\hline Hospitals ............................... & 4,823 \\
\hline Transplant programs ........... & 750 \\
\hline Home Health Agencies & 12,624 \\
\hline \multirow{2}{*}{\multicolumn{2}{|c|}{ Comprehensive Outpatient Reha- }} \\
\hline & \\
\hline Community Mental Health Centers & 52 \\
\hline Portable X-Ray Services .................. & 500 \\
\hline $\begin{array}{l}\text { Rural Health Clinics and Federally } \\
\text { Qualified Health Centers ............. }\end{array}$ & 12,034 \\
\hline $\begin{array}{l}\text { Emergency Preparedness of Pro- } \\
\text { viders and Suppliers }\end{array}$ & \\
\hline $\begin{array}{l}\text { Total Number of Providers } \\
\text { Average Cost Per Provider ..... }\end{array}$ & $\begin{array}{r}121,982 \\
\$ 533\end{array}$ \\
\hline Total One-Time Cost ..................... & $\$ 65,016,406$ \\
\hline
\end{tabular}

n. Effects on Small Entities, Effects on Small Rural Hospitals, Unfunded Mandates, and Federalism

The RFA requires agencies to analyze options for regulatory relief of small entities, if a rule has a significant impact on a substantial number of small entities. For purposes of the RFA, we estimate that almost all health care providers regulated by CMS are small entities as that term is used in the RFA (including small businesses, nonprofit organizations, and small governmental jurisdictions). The great majority of hospitals and most other health care providers and suppliers are small entities, either by being nonprofit organizations or by meeting the SBA definition of a small business (having revenues of less than $\$ 8$ million to $\$ 41.5$ million in any 1 year, varying by type of provider and highest for hospitals). Accordingly, almost all of the savings that the rule will create benefits small entities. We note that individual persons are not small entities for purposes of the RFA, and hence the lifeextending transplantation benefits of the rule are not relevant to the RFA.

The RFA requires that a final regulatory flexibility analysis (FRFA) be prepared if a final rule would have a "significant impact on a substantial number" of such entities. HHS interprets the statute as mandating this analysis only the impact is adverse, though there are differing

interpretations. Regardless, there is no question that the final rule would affect a "substantial number" of small entities. As shown in Table 17, the total number of affected entities will be about 122,000 , including those affected by more than one provision. The rule of thumb used by HHS for determining whether an impact is "significant" is an effect of 3 percent or more of annual revenues. These savings do not approach that threshold. Hospitals account for about one-third of all health care spending and even if all these savings accrued to hospitals this threshold would not be approached. Therefore, the Secretary has determined that these provisions of the final rule will not have a significant economic impact on a substantial number of small entities.

In addition, section 1102(b) of the Social Security Act requires us to prepare a regulatory impact analysis if a rule may have a significant impact on the operations of a substantial number of small rural hospitals. This analysis must conform to the provisions of section 604 of the RFA. For purposes of section 1102(b) of the Act, we define a small rural hospital as a hospital that is located outside of a metropolitan statistical area and has fewer than 100 beds. For the reasons previously given, the Secretary has determined that these provisions of the final rule will not have a significant impact on the operations of a substantial number of small rural hospitals.

Section 202 of the Unfunded Mandates Reform Act of 1995 (UMRA) also requires that agencies assess anticipated costs and benefits before issuing any rule whose mandates require spending in any 1 year of $\$ 100$ million in 1995 dollars, updated annually for inflation. In 2019, that threshold is approximately $\$ 154$ million. These provisions of the final rule contain no mandates that will impose spending costs on State, local, or tribal governments, or on the private sector. Indeed, it substantially reduces existing private sector mandates.

Executive Order 13132 establishes certain requirements that an agency must meet when it promulgates a proposed rule (and subsequent final rule) that imposes substantial direct requirement costs on State and local governments, preempts State law, or otherwise has federalism implications. The final rule imposes no such requirements. Importantly, it would remove Federal requirements setting qualification standards for hospice aides. Setting qualifications for health care workers is traditionally a State function, and this change would therefore remove an infringement on State prerogatives.

o. Effects on Costs to Facilities, Providers, Medicare, Other Insurance, and Patients

Most of the individual proposals addressed in the preceding analysis involve reducing burdensome costs on facilities, health care professionals, and patients. Most of those reductions save time and effort currently performed on tasks that we proposed to eliminate or reform and those reductions will result ultimately in reduced medical care costs in these facilities, some of which will result in further effects on public and private insurance costs. In this regard, it is important to emphasize that the CoPs and CfCs generally apply to all patients served by a Medicare and/or Medicaid participating provider or supplier, not just Medicare or Medicaid patients, and to the entire operations of the provider. Revisions to those requirements apply broadly to the entire health care system. We are hopeful that cost reductions ultimately flow to reductions in charges, to reductions in third party payments, and hence to reductions in insurance costs and to those who pay those costs.

Initial savings will accrue primarily to providers. How much of these savings will flow to insurers and patients depends primarily on the payment and reimbursement mechanisms in place for each affected entity for those particular costs. According to the National Health Expenditure Accounts, approximate payer shares in 2016 were 11 percent for consumer out of pocket, 35 percent for private health insurance, 21 percent for Medicare, 18 percent for Medicaid, and 15 percent for other public and private payers such as the Department of Veteran Affairs and the Department of Defense. We would expect savings to approximate these shares. Ultimately, all costs are paid by workers and taxpayers who pay for all health care directly or indirectly, quite apart from immediate cost subsidies or cost sharing.

Two provisions directly reduce Medicare and other insurance costs. Eliminating unnecessary patient history and physical examinations and medical tests for procedures (such as cataract surgery) performed in ASCs and in hospital outpatient surgery will disproportionately reduce Medicare costs, since use of these services rises with age. Additional transplantation of 
kidneys will reduce Medicare’s ESRD costs, partially offset by increased transplantation costs. Because of the difficulty in finding evidence of the volume of such savings, we cannot estimate the likely effects on Medicare spending.

Most of the facility and provider savings will accrue to Medicare and other insurers over time as payment rate increases are slightly reduced, and the remainder will accrue to other payers and to patients.

\section{p. Benefits to Patients}

We discussed life-extending and lifesaving benefits at length in the analysis of increases in transplantation. These result from removal of disincentives to transplant patients, or to use organs, where this could reduce success rates by a few percent and possibly trigger closure of transplant centers or programs under current rules. As previously explained, we do not have robust estimates. There are additional and substantial patient benefits likely to result from the cost-reducing reforms that we proposed. Time not wasted by medical care providers or facilities on unnecessary tasks is time that can be used to focus on better care. While such effects could be measured in principal, there is little existing data on magnitudes of such effects. We requested but did not receive public comments on these or any other aspects of costs and benefits of the proposed rule.

\section{Alternatives Considered}

From within the entire body of CoPs and CfCs, we selected what we believe to be the most viable candidates for reform as identified by stakeholders, by recent research, or by experts as unusually burdensome. This subset of the universe of standards is the focus of the proposed rule. For all of the proposed provisions, we considered not making these changes. Ultimately, we saw no good reasons not to finalize these burden reducing changes.

We welcomed comments on whether we properly selected the best candidates for change, and welcomed suggestions for additional reform candidates from the entire body of CoPs and other regulatory provisions that fall directly on providers. As discussed earlier in this preamble, we did receive suggestions for additional reforms and will consider those in future reform efforts.

\section{Uncertainty}

Our estimates of the effects of this regulation are subject to significant uncertainty. While the Department is confident that these reforms will provide flexibilities to facilities that will yield major cost savings, there are uncertainties about the magnitude of these effects. Despite these uncertainties, we are confident that the rule will yield substantial overall cost reductions and other benefits. In this analysis we have provided estimates to suggest the potential savings these reforms could achieve under certain assumptions. We appreciate that those assumptions are simplified, and that actual results could be substantially higher or lower. Although there is uncertainty concerning the magnitude of all of our estimates, we do not have the data to provide specific estimates for each reform proposed, as to the range of possibilities, or to estimate all categories of possible benefits, including health effects.

\section{Conclusion}

These provisions of the final rule will substantially reduce existing regulatory requirements imposed on health care providers through the CoPs and related regulatory provisions that Medicare and Medicaid providers must meet. For some provisions, health benefits to patients will be substantial and direct. Other provisions will free up time and efforts of health care providers to focus on improving health care quality and service delivery. Although this rule does not require a final regulatory flexibility analysis, we believe the preceding analysis meets the requirements for such an analysis as set out in $\S 604$ of the Regulatory Flexibility Act. In addition, the analysis above, together with the remainder of this preamble, provides a regulatory impact analysis.

In accordance with the provisions of Executive Order 12866, this regulation was reviewed by the Office of Management and Budget.

B. Regulatory Impact Statement for Fire Safety Requirements for Certain Dialysis Facilities

We have examined the impact of these regulatory provisions as required by Executive Order 12866 on Regulatory Planning and Review (September 30, 1993), Executive Order 13563 on Improving Regulation and Regulatory Review (January 18, 2011), the Regulatory Flexibility Act (RFA) (September 19, 1980, Pub. L. 96-354), section 1102(b) of the Social Security Act, section 202 of the Unfunded Mandates Reform Act of 1995 (March 22, 1995; Pub. L. 104-4), Executive Order 13132 on Federalism (August 4, 1999), the Congressional Review Act (5 U.S.C. 804(2)), and Executive Order 13771 on Reducing Regulation and
Controlling Regulatory Costs (January 30, 2017)

Executive Orders 12866 and 13563 direct agencies to assess all costs and benefits of available regulatory alternatives and, if regulation is necessary, to select regulatory approaches that maximize net benefits (including potential economic, environmental, public health and safety effects, distributive impacts, and equity). A regulatory impact analysis (RIA) must be prepared for major rules with economically significant effects (\$100 million or more in any 1 year).

We do not know how many, if any, dialysis facilities would be affected by this adoption of the 2012 editions of the NFPA 101 and NFPA 99. All States have adopted the 2012 editions, so as a practical matter, all dialysis facilities are already following the 2012

requirements. Therefore, we do not anticipate any impact on the applicable dialysis facilities.

Accordingly, these provisions do not reach the economic threshold and thus are neither economically significant under Executive Order 12866, nor a major rule under the Congressional Review Act.

The RFA requires agencies to analyze options for regulatory relief of small entities, and to prepare a final

regulatory flexibility analysis if a rule is found to have a significant impact on a substantial number of small entities. For purposes of the RFA, small entities include small businesses, nonprofit organizations, and small governmental jurisdictions. Most hospitals and most other providers and suppliers are small entities, either by nonprofit status or by having revenues of less than $\$ 7.5$ million to $\$ 38.5$ million in any 1 year. Individuals and States are not included in the definition of a small entity. We are not preparing a final regulatory flexibility analysis because we have determined, and the Secretary certifies, that these provisions of the final rule will not have a significant economic impact on a substantial number of small entities.

In addition, section 1102(b) of the Social Security Act (the Act) requires us to prepare a regulatory impact analysis if a rule may have a significant impact on the operations of a substantial number of small rural hospitals. This analysis must conform to the provisions of section 604 of the RFA. For purposes of section 1102(b) of the Act, we define a small rural hospital as a hospital that is located outside of a Metropolitan Statistical Area for Medicare payment regulations and has fewer than 100 beds. We are not preparing an analysis for section 1102(b) of the Act because 
we have determined, and the Secretary certifies, that these provisions of the final rule will not have a significant impact on the operations of a substantial number of small rural hospitals.

Section 202 of the Unfunded Mandates Reform Act of 1995 also requires that agencies assess anticipated costs and benefits before issuing any rule whose mandates require spending in any 1 year of \$100 million in 1995 dollars, updated annually for inflation. In 2019, that threshold is approximately $\$ 154$ million. These provisions will have no consequential effect on State, local, or tribal governments or on the private sector.

Executive Order 13132 establishes certain requirements that an agency must meet when it promulgates a proposed rule (and subsequent final rule) that imposes substantial direct requirement costs on State and local governments, preempts State law, or otherwise has Federalism implications. Since these provisions do not impose any costs on State or local governments, the requirements of Executive Order 13132 are not applicable.

In accordance with the provisions of Executive Order 12866, this regulation was reviewed by the Office of Management and Budget.

\section{F. Regulatory Impact Analysis for} Hospital and Critical Access Hospital Changes To Promote Innovation, Flexibility, and Improvement in Patient Care

\section{Statement of Need}

CMS is aware, through conversations with stakeholders and federal partners, and as a result of internal evaluation and research, of outstanding concerns about CoPs for hospitals and CAHs, despite recent revisions. We believe that the revisions will alleviate many of those concerns. In addition, modernization of the requirements would cumulatively result in improved quality of care and improved outcomes for all hospital and $\mathrm{CAH}$ patients. We believe that benefits would include reduced readmissions, reduced incidence of hospital-acquired conditions (including healthcareassociated infections), improved use of antibiotics at reduced costs (including the potential for reduced antibiotic resistance), and improved patient and workforce protections.

These benefits are consistent with former HHS Quality Initiatives, including efforts to prevent HAIs; the national action plan for adverse drug event (ADE) prevention; the national strategy for Combating AntibioticResistant Bacteria (CARB); and the
Department's National Quality Strategy (http://www.ahrq.gov/ workingforquality/index.html). Principles of the National Quality Strategy supported by the proposed rule include eliminating disparities in care; improving quality; promoting consistent national standards while maintaining support for local, community, and Statelevel activities that are responsive to local circumstances; care coordination; and providing patients, providers, and payers with the clear information they need to make choices that are right for them (http://www.ahrq.gov/ workingforquality/nqs/principles.htm). Our proposal to prohibit discrimination would support eliminating disparities in care, and we believe our proposals about QAPI and infection prevention and control and antibiotic stewardship programs will improve quality and promote consistent national standards. Our proposals regarding the term licensed independent practitioners and establishing policies and protocols for when the presence of an $\mathrm{RN}$ is needed will support care coordination and quality of care. In sum, we believe our proposed changes are necessary, timely, and beneficial. We are finalizing most of the aforementioned proposals.

\section{Overall Impact}

We have examined the impacts of this rule as required by Executive Order 12866 on Regulatory Planning and Review (September 30, 1993), Executive Order 13563 on Improving Regulation and Regulatory Review (January 18, 2011), the Regulatory Flexibility Act (RFA) (September 19, 1980, Pub. L. 96354), section 1102(b) of the Social Security Act, section 202 of the Unfunded Mandates Reform Act of 1995 (March 22, 1995; Pub. L. 104-4), Executive Order 13132 on Federalism (August 4, 1999), the Congressional Review Act (5 U.S.C. 804(2)), and Executive Order 13771 on Reducing Regulation and Controlling Regulatory Costs (January 30, 2017).

Executive Orders 12866 and 13563 direct agencies to assess all costs and benefits of available regulatory alternatives and, if regulation is necessary, to select regulatory approaches that maximize net benefits (including potential economic, environmental, public health and safety effects, distributive impacts, and equity). Section 3(f) of Executive Order 12866 defines a "significant regulatory action" as an action that is likely to result in a rule: (1) Having an annual effect on the economy of \$100 million or more in any 1 year, or adversely and materially affecting a sector of the economy, productivity, competition, jobs, the environment, public health or safety, or state, local or tribal governments or communities (also referred to as "economically significant”); (2) creating a serious inconsistency or otherwise interfering with an action taken or planned by another agency; (3) materially altering the budgetary impacts of entitlement grants, user fees, or loan programs or the rights and obligations of recipients thereof; or (4) raising novel legal or policy issues arising out of legal mandates, the President's priorities, or the principles set forth in the Executive Order.

A regulatory impact analysis (RIA) must be prepared for major rules with economically significant effects $(\$ 100$ million or more in any 1 year). We estimate that this rulemaking is "economically significant" as measured by the $\$ 100$ million threshold, and hence also a major rule under the Congressional Review Act. Accordingly, we have prepared a regulatory impact analysis (RIA) that, to the best of our ability, presents the costs and benefits of the rulemaking.

The Congressional Review Act, 5 U.S.C. 801 et. seq., as added by the Small Business Regulatory Enforcement Fairness Act of 1996, generally provides that before a rule may take effect, the agency promulgating the rule must submit a rule report, which includes a copy of the rule, to each chamber of the Congress and to the Comptroller General of the United States. HHS will submit a report containing this rule and other required information to the U.S. Senate, the U.S. House of Representatives, and the Comptroller General of the United States prior to publication of the rule in the Federal Register.

The final rule would create ongoing cost savings to hospitals and CAHs in many areas. We believe these savings would largely, but not necessarily entirely, offset any costs to hospitals and CAHs that would be incurred by other changes we are finalizing in this rule. The financial savings and costs are summarized in Table 18.

We sought public comment on our burden assumptions and estimates as well as comments identifying additional reforms that should be considered for future rulemakings. As is usually the case in impact analysis, substantial uncertainty surrounds these estimates and we solicited comments on any suggestions or data that would inform our estimates for the final rule.

Comment: We received a comment that was generally in support of the changes proposed and the goals of those changes; however, the commenter was concerned that the rule dramatically 
underestimates the time and effort required for compliance with the antibiotic stewardship and Quality Assessment and Performance Improvement (QAPI) programs.

Response: We note that since the QAPI requirement will replace the annual evaluation requirement, we believe many of those resources could be reallocated to QAPI activities to minimize burden. In addition, we have re-evaluated our proposed requirements and eliminated unnecessary prescriptiveness, allowing each CAH the flexibility to implement its QAPI program in the most efficient manner for its unique circumstances. With regards to the antibiotic stewardship program, we have expanded on our cost estimates to account for a more robust intervention, and presented a range of estimates to account for uncertainty.

Comment: We received a comment expressing concern over whether the costs of implementing the infection prevention and control program were underestimated due to the additional training and technical assistance that would be required for the individual fulfilling the infection preventionist role in CAHs.

Response: We thank you for your comment, and we agree that the burden for CAHs due to the infection preventionist role was underrepresented in the proposed rule. We have revised our estimates to account for that error in this final rule.

TABLE 18-SECTION-BY-SECTION ECONOMIC IMPACt ESTIMATES

\begin{tabular}{|c|c|c|c|}
\hline Issue & Frequency & $\begin{array}{l}\text { Number of } \\
\text { affected } \\
\text { entities }\end{array}$ & $\begin{array}{c}\text { Estimated } \\
\text { net costs } \\
(\$ \text { millions })^{*}\end{array}$ \\
\hline \multirow{4}{*}{$\begin{array}{l}\text { Hospitals } \\
\text { Patients' rights (RIA) Nursing services (ICR) } \\
\text { - Nursing services (ICR) } \\
\text { - Infection Prevention \& Control } \\
\text { and Antibiotic Stewardship (RIA) }\end{array}$} & & $\begin{array}{l}4,823 \\
4,823\end{array}$ & \\
\hline & Every 3 years & 1,193 & \\
\hline & 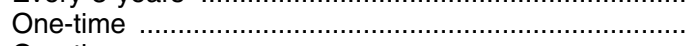 & 1,193 & \\
\hline & One-time $\ldots \ldots \ldots . . .$. & 4,823 & 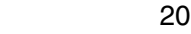 \\
\hline - Food and dietary (RIA) ........................ & Recurring annually .... & 677 & -5 \\
\hline - Infection Prevention \& Control & 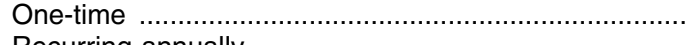 & 1,353 & \\
\hline & Recurring annually & 1,004 & 148 \\
\hline and Antibioti & 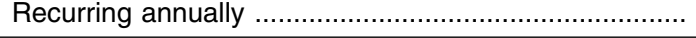 & 501 & -27 \\
\hline
\end{tabular}

Note: This table includes entries only for those proposed reforms that we believe would have a measurable economic effect; includes estimates from ICRs and RIA. Negative costs indicate cost savings.

${ }^{*}$ Amounts rounded to the nearest million.

\section{Anticipated Effects}

There are about 4,823 hospitals and 1,353 CAHs that are certified by Medicare and/or Medicaid. We use these figures to estimate the potential impacts of the final rule. In the estimates that were shown in the Collection of Information Requirements section of the preamble and in the RIA here, we estimate hourly costs as follows. Using May 2017 data from the Bureau of Labor Statistics, we have obtained estimates of the national average hourly wage for all medical professions (https://www.bls.gov/oes/ 2017/may/oes_nat.htm). We have adjusted these rates by adding 100 percent to the hourly wage to account for overhead costs and fringe benefit costs. We use the following average hourly wages in our estimates:

\section{TABLE 19-HOURLY COSTS BY PROFESSION}

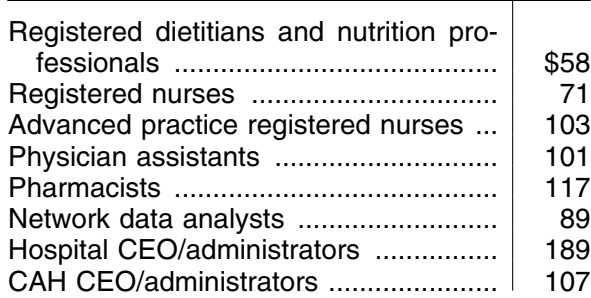

\section{TABLE 19-HOURLY COSTS BY PROFESSION-Continued}

\begin{tabular}{|c|c|}
\hline orkers & 3 \\
\hline & 19 \\
\hline poratory Techni & 5 \\
\hline
\end{tabular}

a. Effects on Hospitals

(1) Licensed Independent Practitioners (Patients' Rights § 482.13)

We are finalizing our proposal to delete the modifying term "independent" from the CoP at $\S 482.13(\mathrm{e})(5)$, as well as at $\S 482.13(\mathrm{e})(8)(\mathrm{ii})$. While we believe that hospitals might be able to achieve some costs savings through these changes (by having additional licensed practitioners such as PAs allowed to write restraint and seclusion orders and thus relieve some of the burden from physicians), we do not have a reliable means of quantifying these possible cost savings. We solicited comments as to whether the assumption of cost savings is reasonable and welcomed any data that may help inform the costs and benefits of this provision, but did not receive any comments specific to our solicitation.
(2) Infection Control and Antibiotic Stewardship (Infection Prevention and Control § 482.42)

We are revising the hospital requirements at 42 CFR 482.42 , "Infection control," which currently require hospitals to provide a sanitary environment to avoid sources and transmission of infections and communicable diseases. Hospitals are also currently required to have a designated infection control officer, or officers, who are required to develop a system to identify, report, investigate and control infections and communicable diseases of patients and personnel. The hospital's CEO, medical staff, and director of nursing services are charged with ensuring that the problems identified by the infection control officer or officers are addressed in hospital training programs and their QAPI program. The CEO, medical staff, and director of nursing services are also responsible for the implementation of successful corrective action plans in affected problem areas.

We are finalizing our proposal to change to the title of this CoP to "Infection prevention and control and antibiotic stewardship programs.” By adding the word "prevention" to the CoP name, our intent is to promote 
larger, cultural changes in hospitals such that prevention initiatives are recognized on balance with their current, traditional control efforts. And by adding "antibiotic stewardship" to the title, we would emphasize the important role that a hospital could play in improving patient care and safety and combatting antimicrobial resistance through implementation of a robust stewardship program that follows nationally recognized guidelines for appropriate antibiotic use. Along with these changes, we proposed to change the introductory paragraph to require that a hospital's infection prevention and control and antibiotic stewardship programs be active and hospital-wide for the surveillance, prevention, and control of HAIs and other infectious diseases, and for the optimization of antibiotic use through stewardship. We will also require that a program demonstrate adherence to nationally recognized infection prevention and control guidelines for reducing the transmission of infections, as well as best practices for improving antibiotic use, for reducing the development and transmission of HAIs and antibioticresistant organisms. While these particular changes are new to the regulatory text, it is worth noting that these requirements, with the exception of the new requirement for an antibiotic stewardship program, have been present in the Interpretive Guidelines (IGs) for hospitals since 2008 (See A0747 at Appendix A-Survey Protocol, Regulations and Interpretive Guidelines for Hospitals, http:/cms.gov/manuals/ Downloads/som107ap_a_hospitals.pdf).

\section{(a) Infection Prevention and Control}

Each hospital will be required to review their current infection control program and compare it to the new requirements contained in this section. After performing this comparison, each hospital will be required to revise their program so that it complies with the requirements in this section. Based on our experience with hospitals, we believe that a physician and a nurse on the infection control team will conduct this review and revision of the program. We believe both the physician and the nurse will spend 16 hours each for a total of 32 hours. According to BLS data, doubled to account for overhead costs and fringe benefits, physician time costs an average of \$191 an hour, and nurses' time costs an average wage of $\$ 71$ an hour. Thus, to ensure their infection control program complied with the requirements in this section, we estimate that each hospital will require 32 burden hours (16 hours for a physician and 16 hours for a nurse) at a cost of $\$ 4,192(\$ 3,056$ (\$191 an hour for a physician $\times 16$ burden hours) + $\$ 1,136$ ( $\$ 71$ an hour for a nurse $\times 16$ burden hours)). Based on the estimate, for all 4,823 hospitals, complying with this requirement will require 154,336 burden hours (32 hours for each hospital $\times 4,823$ hospitals) at a one-time cost of approximately $\$ 20$ million $(\$ 4,192$ for each hospital $\times 4,823$ hospitals).

At $\S 482.42(a)(1)$, we are finalizing our proposal to require the hospital to appoint an infection preventionist(s)/ infection control professional(s). Within this change we are deleting the outdated term, "infection control officer," and replacing it with the more current and accurate terms, "infection preventionist/ infection control professional." CDC has defined "infection control professional (ICP)" as "a person whose primary training is in either nursing, medical technology, microbiology, or epidemiology and who has acquired specialized training in infection control." In designating infection preventionists/ICPs, hospitals should ensure that the individuals so designated are qualified through education, training, experience, or certification (such as that offered by the CBIC, or by the specialty boards in adult or pediatric infectious diseases offered for physicians by the American Board of Internal Medicine (for internists) and the American Board of Pediatrics (for pediatricians).

Since this requirement has been present in the IGs since 2008, we believe that hospitals have been aware of CMS' expectations for the qualifications of infection control officers. The Joint Commission has a similar requirement (TJC Accreditation Standard IC.01.01.01). We believe that hospitals accredited by TJC (over 75 percent of all hospitals (http:// www.jointcommission.org/facts_about hospital_accreditation/)) should already be in compliance, or near compliance, with this requirement. The Joint Commission requires that a hospital identify the individual(s) responsible for its infection prevention and control program, including the individual(s) with clinical authority over the infection prevention and control program. For these reasons, we do not anticipate any new recurring burden to hospitals attributable to appointing an infection preventionist.

\section{(b) Antibiotic Stewardship (AS)}

At $\S 482.42$ (b), we believe that the finalized requirements for a hospital to have an active antibiotic stewardship program, and for its organization and policies, would constitute additional regulatory burden, as will be discussed in more detail below. However, we believe that the estimated costs of an AS program would be greatly offset by the savings that a hospital would achieve through such a program. The most obvious savings would be from decreased inappropriate antibiotic use leading to overall decreased drug costs for a hospital. Antimicrobial costs, particularly antibiotic costs, often constitute a significant percentage of the pharmacy budget for a hospital, so reducing overall antibiotic use would most likely have a substantial impact in lowering overall drug costs for a hospital. In fact, our review of the literature showed significant savings in this area, with annual savings proportional to bed size of the hospital or hospital unit. Reported annual savings ranged from $\$ 27,917$ (Canadian dollars) for a 12-bed medical/surgical intensive care unit to $\$ 2.1$ million for an 880-bed academic medical center. 67

We specifically note the $\$ 177,000$ in annual drug cost savings achieved by a 120-bed community hospital with its AS program for the year of 2000 compared to 1999 , and would use that as the average cost savings for the averagesized 124-bed hospital discussed above (LaRocco 2003, CID “Concurrent antibiotic review programs-a role for infectious diseases specialists at small community hospitals"). Inflating this number to 2017 dollars using the consumer price index yields approximately $\$ 258,000$. According to NHSN survey data, almost 82 percent of hospitals were implementing all 7 of CDC's core elements of hospital antibiotic stewardship programs in 2017. This is significantly higher than the estimate published in the proposed rule, because the number of hospitals implementing AS programs has increased dramatically in the past several years. This is primarily driven by large accrediting organizations announcing and implementing their own antimicrobial stewardship standards. Preliminary 2018 data suggest that this upward trend of AS programs is likely to continue to some degree; however, since the the antimicrobial stewardship standards are already in effect for one of the largest

\footnotetext{
${ }^{6}$ Leung V., Gill S., Sauve J., Walker K., Stumpo C., Powis J. Growing a "positive culture" of antimicrobial stewardship in a community hospital. The Canadian journal of hospital pharmacy. 2011; 64(5):314-20.

${ }^{7}$ Beardsley J.R., Williamson J.C., Johnson J.W., Luther V.P., Wrenn R.H., Ohl C.C. Show me the money: Long-term financial impact of an antimicrobial stewardship program. Infection control and hospital epidemiology: The official journal of the Society of Hospital Epidemiologists of America. 2012; 33(4):398-400.
} 
accrediting bodies as of January 2017, we would expect a sharp decline in the marginal rate of AS implementation in 2017 and beyond without further intervention. Therefore, for our baseline we have projected that approximately $90 \%$ of hospitals would have AS programs in 2018 were the rate of adoption to decrease by half, and we assume that is approximately where the market would level off absent further intervention. We have accounted for this uncertainty by providing estimates in the accounting statement that are 25 percent higher or lower than our primary estimate. Accordingly, we estimate that the annual drug cost savings for 10 percent of all 4,823 hospitals under the rule would be approximately $\$ 124$ million (482 hospitals $\times \$ 258,000$ ). While most of the evidence we reviewed showed clear savings through reduced hospital pharmacy costs that was a direct result of reduced antibiotic use, some studies also demonstrated other financial benefits for hospitals with AS programs, such as decreases in patient length of stay and readmission rates as well as reductions in CDI rates and costs (as we discuss in more detail in the paragraphs to follow).

Based on the published studies that we previously noted, we therefore also believe that the proposed requirement for an AS program would assist hospitals in significantly reducing rates of CDI and the attendant costs. Based on an AS program model developed by the CDC, a hospital combined IC/AS program with an average effectiveness rate of 50 percent would reduce the number of CDIs among Medicare beneficiaries annually by $101,000 .^{8}$ The costs examined in the model were costs for patients who developed CDIs while they were in the hospital or had to be re-admitted to the hospital for a case of CDI that was a result of a recent hospitalization, so the costs are much higher than what would be associated with outpatient cases. The 101,000reduction is an annual reduction in the number of cases of CDI among patients who develop the infection because of medical care; that is, they were admitted for something else and then acquired CDI while getting care. It should be noted that the 101,000 number actually comprises two types of CDI-cases that occur while the patient is in the hospital

${ }^{8}$ Rachel B. Slayton, Ph.D., MPH; R. Douglas Scott II, Ph.D.; James Baggs, Ph.D.; Fernanda C. Lessa, MD; L. Clifford McDonald, MD; John A. Jernigan, MD. "The Cost-Benefit of Federal Investment in Preventing Clostridium difficile Infections through the Use of a Multifaceted Infection Control and Antimicrobial Stewardship Program," Infection Control \& Hospital Epidemiology 2015;00(0):1-7. and cases that are directly attributable to a recent hospitalization, but which manifest after the patient is discharged and requires a readmission. Their study found that the cost for patients who develop the infection while they are already in the hospital is between $\$ 4,323$ and $\$ 8,146$. However, the infections related to a recent hospital stay that require readmission are more expensive, on average, because they require an entirely new admission; the cost of those cases is between $\$ 7,061$ and $\$ 11,601$. A more recent CDC study found the attributable patient cost savings for health care-associated clostridioides difficile (HCA-CDI) to be $\$ 6,844$ per prevented case, and $\$ 12,703$ per prevented case of recurrent CDI (2015 \$). ${ }^{9}$ Inflating these numbers to 2017 dollars using the consumer price index returns approximately $\$ 7,133$ and $\$ 13,240$ respectively. Scott et al. built their economic model with a range from 10 to 50 percent effectiveness, which represents a range of between

approximately 200,000 and 1.1 million inpatient cases of HCA-CDI averted in a 5 year period.

For our purposes, we have based our central estimate on the middle of the aforementioned range, or approximately 30 percent effectiveness, resulting in 546,000 inpatient cases of HCA-CDI averted, and 117,000 recurrent cases averted. It is not clear exactly how many of these averted cases would occur in hospitals versus CAHs, but the prevalence of existing AS programs (or lack thereof) suggest CAHs may have more potential for improvement despite their smaller number of beds; there is also a limited amount of research that suggests the rate of CDI may be higher in hospitals with fewer beds, possibly due to rates of testing or other factors; and it is also possible that CAHs serve an older population that is more at risk for healthcare-associated infections than patients at non-CAHs. ${ }^{1011}$ Therefore, we assume an equal number of cases averted per facility, meaning approximately 78 percent of these would occur in hospitals and 22 percent in CAHs. As previously explained, we

${ }^{9}$ R. Douglas Scott II, Rachel B. Slayton, Fernanda C. Lessa, James Baggs, Steven D. Culler, L. Clifford McDonald and John A. Jernigan; "Assessing the social cost and benefits of a national requirement establishing antibiotic stewardship programs to prevent Clostridioides difficile infection in US hospitals"; Antimicrobial Resistance \& Infection Control, 2019, 8:17.

${ }^{10}$ Kamboj M, Brite J, Aslam A, et al. Artificial Differences in Clostridium difficile Infection Rates Associated with Disparity in Testing. Emerging Infectious Diseases. 2018;24(3):584-587.

${ }^{11}$ Joynt, K. E., Harris, Y., Orav, E. J., \& Jha, A. K. (2011). Quality of care and patient outcomes in critical access rural hospitals. JAMA, 306(1), 45-52. doi:10.1001/jama.2011.902. estimate that 90 percent of hospitals already have AS programs, and therefore 10 percent of those averted cases would be attributable to this regulation. This comes to a total of 42,588 HCA-CDI cases averted, and 9,126 recurrent cases averted for hospitals in a 5 year period. Multiplying these averted cases by the attributable patient cost savings, and annualizing the amount, comes to approximately $\$ 85$ million in annualized patient cost savings. These patient cost savings do not include the cost savings attributable to any averted or modified antibiotic regimen, which was calculated above.

Thus, we estimate that the combined annual drug cost savings and patient cost savings will be approximately $\$ 209$ million. These savings will accrue to patients (reduced out-of-pocket costs), hospitals (reduced costs and improved balance sheets), as well as healthcare insurers, including Medicare (over time, payment rates will be adjusted downward as hospital costs fall). However, we are not able to apportion the savings that would accrue to each group with any accuracy and it will inevitably change over time as insurance rates change. Regardless, healthcare-associated infections are known to be expensive to insurers, including CMS. Preventing these infections will reduce CMS and other insurer expenditures, both on direct hospital costs and through reduced readmissions. The cost-savings estimates for CDI included in the RIA provide an example of the savings Medicare and other insurers could realize through reductions in just one HAI. Ultimately, of course, insurance costs (and the medical care they pay for) are paid by taxpayers and workers. Even the employer contribution to insurance costs is generally regarded by economists as part of worker compensation. We requested comment regarding data that would allow for more robust quantification of the rule's impacts on HAIs other than CDI, but did not receive any such comments.

We believe that the burden of implementing and maintaining an AS program includes the costs of the qualified personnel needed to establish and manage such a hospital program. In the proposed rule, our review of the literature, consultations with CDC, and experience with hospitals suggested that the establishment and maintenance of a hospital antibiotic stewardship program for an average-size hospital (approximately 124 beds), would require at least the leadership of a physician (preferably one with training in infectious diseases) and a clinical pharmacist, and also the services of a 
network data analyst, at the following proportions of full-time employee salaries respectively: $0.10,0.25$, and 0.05. However, the latest research on the resources required for an effective AS program suggest that the minimum fulltime equivalent support recommended for a hospital of this size may be somewhat more burdensome, due to the leadership of a pharmacist and physician at the full time equivalents of their salaries of 1.0 and 0.4 respectively. ${ }^{12}$ We also based our estimates on the prior assumption that 10 percent of hospitals do not yet have programs that implement all of the CDC core elements. Based on these assumptions, the minimum annual cost of the essential team members for a hospital to establish and maintain an antibiotic stewardship program would be $\$ 386,800((\$ 191 \times 0.40 \times 2,000$ hours per year $=\$ 152,800$ for a physician $)+$ $(\$ 117 \times 1.00 \times 2,000$ hours per year $=$ $\$ 234,000$ for a clinical pharmacist)). The annual labor cost for 10 percent of hospitals $(\$ 386,800 \times 482)$ would be approximately $\$ 186$ million.

We invited public comment regarding the amount by which costs may exceed savings in cases of non-voluntary IC/AS program adoption, but did not receive comments with specific estimates.

\section{b. Effects on CAHs}

(1) Ordering Privileges for Qualified Dietitians (RDs) and Qualified Nutrition Professionals (Provision of Services $\S 485.635$ )

We are finalizing our proposal to revise the $\mathrm{CAH}$ requirements at $42 \mathrm{CFR}$ 485.635 (a)(3)(vii), which currently require that the nutritional needs of inpatients are met in accordance with recognized dietary practices and the orders of the practitioner responsible for the care of the patients. Specifically, we proposed revisions that would change the CMS requirements to allow for flexibility in this area by requiring that all patient diets, including therapeutic diets, must be ordered by a practitioner responsible for the care of the patient, or by a qualified dietitian or qualified nutrition professional as authorized by the medical staff in accordance with State law governing dietitians and nutrition professionals.

With these changes to the current requirements, a CAH will have the

12 Sarah B Doernberg, Lilian M Abbo, Steven D Burdette, Neil O Fishman, Edward L Goodman, Gary R Kravitz, James E Leggett, Rebekah W Moehring, Jason G Newland, Philip A Robinson, Emily S Spivak, Pranita D Tamma, Henry F Chambers, Essential Resources and Strategies for Antibiotic Stewardship Programs in the Acute Care Setting, Clinical Infectious Diseases, Volume 67 Issue 8, 15 October 2018, Pages 1168-1174 regulatory flexibility to grant qualified dietitians/nutrition professionals specific dietary ordering privileges (including the capacity to order specific laboratory tests to monitor nutritional interventions and then modify those interventions as needed). We believe that this is another area of change to the requirements that might produce savings since this will allow physicians to delegate to a qualified dietitian or qualified nutrition professional the task of prescribing patient diets, including therapeutic diets, to the extent allowed by state law. We further believe that dietitians or other clinically qualified nutrition professionals are already performing patient dietary assessments and making dietary recommendations to the physician (or PA or APRN) who then evaluates the recommendations and writes orders to implement them. Our analysis does not take into account improved quality of life nor improved clinical outcomes for the patient. We do not currently have data to more precisely estimate the savings that this revision could produce in CAHs. However, we believe that it might allow for better use of both physician/PA/ APRN and dietitian/nutrition professional time and could result in improved quality of life and improved clinical outcomes for CAH patients.

More obviously, dietitians/nutrition professionals with ordering privileges will be able to provide dietary/ nutritional services at lower costs than physicians (as well as APRNs and PAs, two categories of non-physician practitioners that have traditionally also devised and written patient dietary plans and orders). This cost savings stems in some part from significant differences in the average salaries between the professions and the time savings achieved by allowing dietitians/ nutrition professionals to autonomously plan, order, monitor, and modify services as needed and in a more complete and timely manner than they are currently allowed. Savings would be realized by CAHs through the physician/APRN/PA time and salaries saved.

Physicians, APRNs, and PAs often lack the training and educational background to manage the nutritional needs of patients with the same efficiency and skill as dietitians/ nutrition professionals. The addition of ordering privileges enhances the ability that dietitians/nutrition professionals already have to provide timely, costeffective, and evidence-based nutrition services as the recognized nutrition experts on a $\mathrm{CAH}$ interdisciplinary team.
It might seem natural to calculate these cost savings for CAHs based on the following assumptions:

- There is an average hourly cost difference of $\$ 74$ between dietitians/ nutrition professionals on one side (\$58 per hour) and the hourly cost average for physicians, APRNs, and PAs (\$132 per hour) on the other;

- There were 282,584 inpatient visits by Medicare beneficiaries in 2011 (According to a December 2013 OIG report (http://oig.hhs.gov/oei/reports/ oei-05-12-00081.pdf)) with each of these stays requiring at least one dietary plan and orders;

- On average, each dietary order, including ordering and monitoring of laboratory tests, subsequent modifications to orders, and dietary orders for discharge/transfer/outpatient follow-up as needed, will take 30 minutes (0.5 hours) of a physician's/ APRN's/PA's/dietitian's/nutrition professional's time per patient during an average stay; and

- We estimate that approximately 50 percent of CAHs (or approximately 677 CAHs) have not already granted ordering privileges to dietitians and nutrition professionals, reducing the number of total number of CAH inpatient stays to 141,292 .

The resulting savings would be $\$ 7,722$ annually on average for each $\mathrm{CAH}$ $(141,292$ inpatient hospital stays $\times 0.50$ hours of a physician's/APRN's/PA's/ dietitian's/nutrition professional's time $\times \$ 74$ per hourly cost difference $\div 677$ CAHs) for a total annual savings of approximately $\$ 5.2$ million. We note that these estimates exclude some categories of cost increases (for example, internal CAH meetings to plan changes and the time and other costs of training physicians, dietitians/nutrition professionals, and other staff on the new dietary ordering procedures). Even more importantly, this estimate does not account for barriers, other than federal regulation, to $\mathrm{RDs}$ receiving ordering privileges; Weil et al. (2008) provide evidence on the existence of such barriers, which would likely prevent at least some of these cost savings from being realized. ${ }^{13}$ If such barriers are not relevant, then there is another adjustment that would need to be made to the calculation. Specifically, the dietitian wage estimate would need to be revised because the wage data do not account for the increase in demand for dietitians we projected would result from the hospital burden reduction rule

\footnotetext{
${ }^{13}$ Weil, Sharon D., et al. "Registered Dietitian Prescriptive Practices in Hospitals." Journal of the American Dietetic Association 108:1688-1692. October 2008.
} 
finalized that same month. For the savings estimates accompanying that rule to be achieved would require at least 6.7 percent of the dietitian FTEs in the U.S. to be newly allocated to providing nutrition services to hospital patients. ${ }^{14}$ This shift in activity entails a substantial movement along the supply curve for dietitian labor, thus raising the dietitian wage and reducing the cost savings estimated with the method outlined. For these reasons, as well as our lack of data on CAH outpatient visits for nutritional services and the impact that the proposed regulatory changes might have on hospital costs in this area, we present the estimate for discussion purposes only.

(2) $\$ 485.640$ Condition of Participation: Infection Prevention and Control and Antibiotic Stewardship Programs

As we finalized for hospitals, we are also finalizing the new infection prevention and control and antibiotic stewardship requirements for CAHs. The infection control requirements for CAHs have remained unchanged since 1997. We are adding a new infection prevention and control (as well as antibiotic stewardship) CoP for CAHs because the existing standards for infection control do not reflect the current nationally recognized practices for the prevention and elimination of healthcare-associated infections.

\section{(a) Infection Prevention and Control}

Each CAH will be required to review their current infection control program and compare it to the new requirements. After performing this comparison, each $\mathrm{CAH}$ will be required to revise their program so that it complies with the new requirements. Based on our experience with CAHs, we believe that a physician and a nurse on the infection control team would conduct this review and revision of the program. We believe both the physician and the nurse will spend 16 hours each for a total of 32 hours. Physicians earn an average of $\$ 191$ an hour, and nurses earn an average wage of $\$ 71$ an hour, including

${ }^{14}$ BLS data show employment of 59,490 dietitians, with a mean hourly wage of $\$ 27.62$. Assuming all dietitians are employed full-time $(2,080$ hours annually) yields a total sector value of $\$ 3.4$ billion, or $\$ 6.8$ billion when doubled to account for fringe benefits and overhead. For the May, 2014, final rule, we estimated $\$ 459$ million of loaded wage savings associated with dietary ordering switching from physicians, nurse practitioners and physician assistants to lower-paid dietitians. Thus the relevant portion of the savings estimate equals roughly 6.7 percent $(=\$ 459$ million $\div \$ 6.8$ billion) of the sector as a whole-and would exceed 6.7 percent, to the extent that some current dietitian positions are part-time. overhead and fringe benefits. Thus, to ensure their infection control program complies with the new requirements, we estimate that each $\mathrm{CAH}$ would require 32 burden hours (16 hours for a physician and 16 hours for a nurse) at a cost of $\$ 4,192(\$ 3,056$ ( $\$ 191$ an hour for a physician $\times 16$ burden hours) + $\$ 1,136$ ( $\$ 71$ an hour for a nurse $\times 16$ burden hours)). Based on the estimate, for all 1,353 CAHs, complying with this requirement will require 43,296 burden hours (32 hours for each CAH $\times 1,353$ CAHs) at a one-time cost of approximately $\$ 5.7$ million $(\$ 4,192$ for each $\mathrm{CAH} \times 1,353 \mathrm{CAHs}$ ).

CAHs will also incur a recurring cost due to the new requirement that they appoint an infection preventionist. The Joint Commission has a similar requirement (TJC Accreditation Standard IC.01.01.01), and so we believe that the 349 CAHs accredited by TJC should already be in compliance, or near compliance, with this requirement. The Joint Commission requires that a $\mathrm{CAH}$ identify the individual(s) responsible for its infection prevention and control program, including the individual(s) with clinical authority over the infection prevention and control program. For the remaining CAHs not accredited by TJC, we are calculating the burden for them to come into compliance with this requirement.

Based on our experience with CAHs, we believe that most ICPs would be registered nurses with experience, education, and training in infection control. As of 2017, approximately 1,004 CAHs are not accredited by TJC. For the purposes of a burden estimate, we assume that each CAH will choose to employ one ICP full-time (52 weeks $\times 40$ hours $=2,080$ hours) at $\$ 71$ per hour, although the regulation does not require the hiring of a new individual, and this position and its associated burden may overlap with that calculated for antibiotic stewardship below. Nonetheless, the cost per facility is estimated to be $\$ 147,680$ annually $(2,080$ hours $\times \$ 71)$, and the total cost for all non-TJC-accredited CAHs would be approximately $\$ 148$ million annually $(1,004 \times \$ 147,680)$.

\section{(b) Antibiotic Stewardship}

Similarly, we believe that the finalized requirements for a $\mathrm{CAH}$ to have an active antibiotic stewardship program, and for its organization and policies, would constitute additional regulatory burden. We believe that the burden of implementing and maintaining an AS program includes the costs of the qualified personnel needed to establish and manage such a $\mathrm{CAH}$ program. In the proposed rule, our review of the literature, consultations with CDC, and experience with CAHs suggested that the establishment and maintenance of a CAH antibiotic stewardship program for a statutorily mandated 25-bed $\mathrm{CAH}$, would require at least the leadership of a physician (preferably an infectious disease physician or physician with training in antibiotic stewardship) and a clinical pharmacist (preferably with training in infectious diseases or antibiotic stewardship), and also the services of a network data analyst at the following proportions of full-time employee salaries respectively: 0.05, 0.10, 0.025 . However, the latest research on the resources required for an effective AS program suggest that the minimum fulltime equivalent support needed for a CAH may be somewhat more

burdensome. Doernberg et al. were unable to make specific recommendations for hospitals with fewer than 100 beds, however, the average self-reported burden for hospitals under 100 beds was larger than we anticipated. Therefore, for our purposes we assume 25-bed CAHs will incur half of the average costs that were reported by hospitals with fewer than 100 beds. Thus, we estimate each CAH will require the leadership of a pharmacist and physician at the full time equivalents of their salaries of 0.45 and 0.19 respectively. According to NHSN survey data, approximately 58 percent of CAHs reported having an AS program that meets all of the CDC's core elements in 2017. As previously mentioned, this number is significantly higher than the estimate published in the proposed rule because the number of CAHs implementing AS programs has increased dramatically in the past several years. This is primarily driven by large accrediting organizations announcing and implementing their own antimicrobial stewardship standards. Preliminary 2018 data suggest that this upward trend of AS programs is likely to continue to some degree; however, since the antimicrobial stewardship standards are already in effect for one of the largest accrediting bodies as of January 2017, we would expect a sharp decline in the marginal rate of AS implementation in 2017 and beyond without further intervention. Therefore, for our baseline we have projected that approximately $63 \%$ of CAHs would have AS programs in 2018 were the rate of adoption to decrease by half, and we assume that is approximately where the market would level off absent further intervention. We have accounted for this uncertainty by providing estimates in the accounting 
statement that are 25 percent higher or lower than our primary estimate. Accordingly, we estimate that approximately 501 CAHs (or 37 percent) have not implemented an AS program. Based on these assumptions, the minimum annual cost of the essential team members for a CAH to establish and maintain an antibiotic stewardship program would be $\$ 177,880$ ( $\$ 191$ per hour $\times 0.19 \times 2,000$ hours per year $=$ $\$ 72,580$ for a physician $)+(\$ 117$ per hour $\times 0.45 \times 2,000$ hours per year $=$ $\$ 105,300$ for a clinical pharmacist). The annual labor cost for 37 percent of CAHs $(\$ 177,880 \times 501)$ would be approximately $\$ 89$ million.

However, we believe that the estimated costs of an AS program would be somewhat offset by the savings that a CAH would achieve through such a program. The most obvious savings would be from decreased inappropriate antibiotic use leading to overall decreased drug costs for a CAH. Our review of the literature showed significant savings in this area, with annual savings proportional to bed size of the hospital. Reported annual savings ranged from $\$ 27,917$ for a 12 -bed medical/surgical intensive care unit to $\$ 2.1$ million for an 880-bed academic medical center. We specifically note the $\$ 177,000$ in annual drug cost savings achieved by a 120-bed community hospital with its AS program for the year of 2000 compared to 1999 (LaRocco 2003, CID “Concurrent antibiotic review programs-a role for infectious diseases specialists at small community CAHs"'), and would use that as the basis to calculate average annual cost savings for a 25-bed CAH. Inflating this number to 2017 dollars using the consumer price index yields approximately $\$ 258,000$. Therefore, (\$258,000 annual savings : 120 beds $=\$ 2,150$ annual cost savings per bed) at $\$ 53,750$ per CAH $(\$ 2,150$ annual cost savings $\mathrm{x} 25$ beds). Using this assumption, we believe that the annual drug cost savings for 37 percent of all 1,353 CAHs under the rule will be approximately $\$ 27$ million (501 CAHs $\times$ $\$ 53,750$ in drug cost savings).

As previously explained, patient cost savings for CAHs has been estimated based on data from Scott et al., and we assume approximately $22 \%$ of HCA-CDI and recurrent cases averted would occur in CAHs. Based on the estimated 63 percent of CAHs that already have AS programs, approximately 37 percent of those averted cases would be attributable to this regulation. This comes to a total of 44,444 HCA-CDI cases averted, and 9,524 recurrent cases averted for CAHs in a 5 year period. Multiplying these averted cases by the attributable patient cost savings, and annualizing the amount, comes to approximately $\$ 89$ million in annualized patient cost savings.

Accordingly, we estimate that the combined annual drug cost savings and patient cost savings will be approximately \$116 million. These savings will accrue to patients (reduced out-of-pocket costs), CAHs (reduced costs and improved balance sheets), as well as healthcare insurers, including Medicare (over time, payment rates will be adjusted downward as CAH costs fall). However, we are not able to apportion the savings that would accrue to each group with any accuracy and it will inevitably change over time as insurance rates change. Regardless, healthcare-associated infections are known to be expensive to insurers, including CMS. Preventing these infections will reduce CMS and other insurer expenditures, both on direct hospital costs and through reduced readmissions. The cost-savings estimates for CDI included in the RIA provide an example of the savings Medicare and other insurers could realize through reductions in just one HAI. Ultimately, of course, insurance costs (and the medical care they pay for) are paid by taxpayers and workers. Even the employer contribution to insurance costs is generally regarded by economists as part of worker compensation.

\section{c. Effects on Patients}

As previously mentioned, some of the estimated cost savings will accrue to patients due to decreased morbidity and associated health care costs. Although this RIA has mainly focused on the costs associated with CDI, there will be savings associated with other infections, such as staphylococcus aureus, that we have not quantified here. Nor have we quantified any savings to patients due to averted travel costs for medical appointments, reduced absence from work, or other miscellaneous costs that would be evaded by patients.

Antibiotic stewardship and infection control has also been proven to significantly reduce morbidity and mortality due to healthcare associated infections. Research by Scott et al., which has been referenced throughout this RIA, thoroughly explored the social costs and benefits of a national requirement establishing antibiotic stewardship programs to prevent CDI. The direct applicability of their study to this RIA is hindered only by differing methods of counting the effects of antimicrobial resistance and infection control. Their study quantifies the effects of AS on the entirety of hospitals with the argument that without these finalized requirements, there would be nothing holding hospitals accountable for maintaining their AS programs.

However, this RIA takes into account a baseline of the current market conditions, which we believe have been strengthened by new standards set by large accrediting bodies. Nonetheless, they estimate CDI infection prevention alone to avert as many as 1.1 million inpatient cases and 44,000 deaths at a 3 percent discount rate over a 5 year period. Using estimates for quality adjusted life years, their central estimate for the value of morbidity risk reduction at a 3 percent discount rate is as much as $\$ 3$ billion worth of lost quality adjusted life years from HCA-CDI, and their central estimate for the benefits of mortality risk reduction is as much as $\$ 401$ billion utilizing estimates for the value of a statistical life.

\section{d. Effects on Small Entities}

The RFA requires agencies to analyze options for regulatory relief of small entities, if a rule has a significant impact on a substantial number of small entities. For purposes of the RFA, we estimate that the great majority of the providers that would be affected by CMS rules are small entities as that term is used in the RFA. The great majority of hospitals and most other healthcare providers and suppliers are small entities, either by being nonprofit organizations or by meeting the SBA definition of a small business.

Accordingly, the usual practice of HHS is to treat all providers and suppliers as small entities in analyzing the effects of our rules.

These provisions of the final rule are anticipated to cost CAHs as much as $\$ 119$ million in the first year. While this is a large amount in total, the average cost per affected CAH is approximately $\$ 88,000$ in the first year, and slightly less in future years. Although the overall magnitude of the paperwork, staffing, and related cost reductions to hospitals and CAHs under this rule is economically significant, the net effect is likely to be a fraction of 1 percent of total hospital costs. Total national hospital care expenditure are approximately 1,143 billion dollars a year, or an average of about $\$ 185$ million per hospital, and our primary estimate of the net effect of these proposals on hospital costs is approximately $\$ 79$ million annually.

Under HHS guidelines for regulatory flexibility analyses, actions that do not negatively affect costs or revenues by more than 3 percent a year are generally not considered to be significant. We do not believe that hospitals of any size will be negatively affected to this 
degree. Accordingly, we have determined that the rule will not have a significant economic impact on a substantial number of small entities, and certify that a final regulatory flexibility analysis is not required. Notwithstanding this conclusion, we believe that this RIA and the preamble as a whole meet the requirements of the RFA for a final regulatory flexibility analysis.

In addition, section 1102(b) of the Act requires us to prepare a regulatory impact analysis if a rule may have a significant impact on the operations of a substantial number of small rural hospitals. This analysis must conform to the provisions of section 604 of the RFA. For purposes of section 1102(b) of the Act, we define a small rural hospital as a hospital that is located outside of a metropolitan statistical area and has fewer than 100 beds. For the preceding reasons, we have determined that these provisions of the final rule will not have a significant negative impact on the operations of a substantial number of small rural hospitals.

Section 202 of the Unfunded Mandates Reform Act of 1995 (UMRA) also requires that agencies assess anticipated costs and benefits before issuing any rule whose mandates require spending in any 1 year of $\$ 100$ million in 1995 dollars, updated annually for inflation. In 2019, that is approximately \$154 million. These provisions of the final rule do contain private sector mandates, but their costs are generally anticipated to be mostly offset by savings. Nevertheless, this RIA and the preamble, taken together, would meet the requirements of an analysis under section 202.

Executive Order 13132 establishes certain requirements that an agency must meet when it issues a proposed rule (and subsequent final rule) that would impose substantial direct requirement costs on State and local governments, preempts State law, or otherwise has Federalism implications. These provisions of the rule will not have a substantial direct effect on State or local governments, preempt State law, or otherwise have federalism implications.

\section{Alternatives Considered}

As we stated, CMS is aware, through conversations with stakeholders and federal partners, and as a result of internal evaluation and research, of outstanding concerns about the CoPs for hospitals and CAHs, despite recent revisions. This subset of the universe of standards is the focus of the final rule.

One alternative we did consider was combining the infection prevention and control leader position with that of the antibiotic stewardship leader position. While this would certainly reduce the costs for hospitals by eliminating one of these positions, we also believe that it might reduce the overall effectiveness of the program and, thus, the overall societal benefits that might be achieved. The skills needed to lead each program are different. Infection prevention programs are often led by nursing staff who do not prescribe antibiotics. Antibiotic stewardship programs are led by physicians and pharmacists who have direct knowledge and experience with antibiotic prescribing. For these reasons, we decided to finalize the requirement as it is contained in this rule.

For all of the finalized provisions, we considered not making these changes. Ultimately, based on our analysis of these issues and for the reasons stated in this preamble, we believe that it is best to propose changes at this time. We welcomed comments on whether we properly selected the best candidates for change, and welcomed suggestions for additional reform candidates from the entire body of CoPs.

\section{Conclusion}

The financial impact of these provisions of the final rule will lie primarily with the balance between estimated costs and savings for the antibiotic stewardship program for hospitals. Nevertheless, the total costs of these provisions are anticipated to be mostly offset by savings. Moreover, the life-saving benefits of some of these provisions, including antibiotic stewardship, have been thoroughly studied and substantiated by independent researchers. However, we note that although savings and morbidity/mortality risk reductions on average are consistent with the literature we've reviewed, the outcomes for individual hospitals and CAHs will vary depending on their specific implementation strategies for AS.

In accordance with the provisions of Executive Order 12866, this regulation was reviewed by the Office of Management and Budget.

\section{Accounting Statement and Table}

As required by OMB Circular A-4 (available at https:// www.whitehouse.gov/sites/ whitehouse.gov/files/omb/circulars/A4/ $a-4 . p d f$ ), in Table 20, we have prepared an accounting statement showing the classification of the transfers and costs associated with the various provisions of the final rule.

While most provisions of the final rule have clearly predictable effects we do not in most cases have detailed empirical information on the precise magnitude of efforts involved (for example, time spent in meeting paperwork or other administrative tasks that apply to a particular provider type). Other provisions (notably those related to organ transplantation and removal of strict $\mathrm{H} \& \mathrm{P}$ requirements before ambulatory or outpatient surgery) have even more uncertain effect sizes. Therefore, we have estimated an upper and lower level for benefit and cost reduction estimates that is 25 percent higher or lower than our primary estimate for all quantified reforms other than those related to ambulatory surgery, and in that area our upper bound for costs is zero cost reductions and our lower bound is a 17 percent reduction in H\&P and associated laboratory testing costs.

TABle 20-Accounting Statement: Classification of Estimated Benefits and SAVINGS [\$ millions]

\begin{tabular}{c|c|c|c|c|c|c}
\hline \multirow{2}{*}{ Category } & Primary & \multirow{2}{*}{$\begin{array}{c}\text { Low } \\
\text { estimate }\end{array}$} & $\begin{array}{c}\text { High } \\
\text { estimate }\end{array}$ & $\begin{array}{c}\text { Year } \\
\text { dollars }\end{array}$ & $\begin{array}{c}\text { Discount } \\
\text { rate }\end{array}$ & $\begin{array}{c}\text { Period } \\
\text { covered }\end{array}$ \\
\hline
\end{tabular}

Benefits:

\begin{tabular}{|c|c|}
\hline Life-Extending Benefits (monetized) & Not Quantified \\
\hline Medical Cost Reduction Benefits (monetized) & Not Quantified \\
\hline
\end{tabular}


TABle 20-Accounting Statement: Classification of Estimated Benefits and SAVINGS-Continued

[\$ millions]

\begin{tabular}{|c|c|c|c|c|c|c|}
\hline \multirow[b]{2}{*}{ Category } & \multirow{2}{*}{$\begin{array}{l}\text { Primary } \\
\text { estimate }\end{array}$} & \multirow{2}{*}{$\begin{array}{c}\text { Low } \\
\text { estimate }\end{array}$} & \multirow{2}{*}{$\begin{array}{c}\text { High } \\
\text { estimate }\end{array}$} & \multicolumn{3}{|c|}{ Units } \\
\hline & & & & $\begin{array}{l}\text { Year } \\
\text { dollars }\end{array}$ & $\begin{array}{l}\text { Discount } \\
\text { rate }\end{array}$ & $\begin{array}{l}\text { Period } \\
\text { covered }\end{array}$ \\
\hline Qualitative ................. & \multicolumn{6}{|c|}{ Potential reductions in morbidity and mortality for hospital and CAH patients } \\
\hline $\begin{array}{l}\text { Costs: } \\
\quad \begin{array}{l}\text { Annualized Monetized Costs }(+) \\
\text { year) }\end{array}\end{array}$ & $\begin{array}{l}-801 \\
-803\end{array}$ & $\begin{array}{l}-1,127 \\
-1,128\end{array}$ & $\begin{array}{l}-485 \\
-487\end{array}$ & $\begin{array}{l}2017 \\
2017\end{array}$ & $\begin{array}{l}7 \% \\
3 \%\end{array}$ & $\begin{array}{l}2019-2028 \\
2019-2028\end{array}$ \\
\hline Transfers & \multicolumn{6}{|c|}{ None } \\
\hline
\end{tabular}

\section{E. Regulatory Reform Analysis Under} E.O. 13771

Executive Order 13771, titled Reducing Regulation and Controlling Regulatory Costs, was issued on January 30,2017 and requires that the costs associated with significant new regulations "shall, to the extent permitted by law, be offset by the elimination of existing costs associated with at least two prior regulations." This final rule is considered an E.O. 13771 deregulatory action. We estimate that this rule generates $\$ 647$ million in annualized cost savings in 2016 dollars, discounted at 7 percent relative to year 2016, over a perpetual time horizon.

Details on the estimated cost savings of this rule can be found in the preceding analyses.

\section{List of Subjects}

42 CFR Part 403

Grant programs-health, Health insurance, Hospitals, Intergovernmental relations, Medicare, Reporting and recordkeeping requirements.

\section{CFR Part 416}

Health facilities, Health professions, Medicare, Reporting and recordkeeping requirements.

\section{CFR Part 418}

Health facilities, Hospice care, Medicare, Reporting and recordkeeping requirements.

\section{CFR Part 441}

Aged, Family planning, Grant programs-health, Infants and children, Medicaid, Penalties, Reporting and recordkeeping requirements.

\section{CFR Part 460}

Aged, Health care, Health records, Medicaid, Medicare, Reporting and recordkeeping requirements.

\section{CFR Part 482}

Grant program-health, Hospitals, Medicaid, Medicare, Reporting and recordkeeping requirements.

\section{CFR Part 483}

Grant programs-health, Health facilities, Health professions, Health records, Medicaid, Medicare, Nursing home, Nutrition, Reporting and recordkeeping requirements, Safety.

\section{CFR Part 484}

Health facilities, Health professions, Medicare, Reporting and recordkeeping requirements.

\section{CFR Part 485}

Grant programs-health, Health facilities, Medicaid, Reporting and recordkeeping requirements.

\section{CFR Part 486}

Grant programs-health, Health facilities, Medicare, Reporting and recordkeeping requirements, X-rays.

\section{CFR Part 488}

Administrative practice and procedures, Health facilities, Health professions, Medicare, Reporting and recordkeeping requirements.

\section{CFR Part 491}

Grant programs-health, Health facilities, Medicaid, Medicare, Reporting and recordkeeping requirements, Rural and urban areas.

\section{CFR Part 494}

Diseases, Health facilities, Incorporation by Reference, Medicare, Reporting and recordkeeping requirements.

For the reasons set forth in the preamble, the Centers for Medicare \& Medicaid Services amends 42 CFR chapter IV as set forth below:

\section{PART 403-SPECIAL PROGRAMS AND PROJECTS}

1. The authority citation for part 403 is revised to read as follows:

Authority: 42 U.S.C. 1302 and 1395hh.

- 2. Section 403.736 is amended by-

a. Removing the introductory text;

- b. Revising paragraph (a);

- c. Removing paragraph (b); and

- d. Redesignating paragraphs (c) and

(d) as paragraphs (b) and (c).

The revision reads as follows:

\section{§403.736 Condition of participation:} Discharge planning.

(a) Discharge planning and instructions. The RNHCI must have in effect a discharge planning process that applies to all patients. The process must assure that appropriate post-institution services are obtained for each patient, as necessary. The RNHCI must assess the need for a discharge plan for any patient likely to suffer adverse consequences if there is no planning.

(1) Discharge instructions must be provided at the time of discharge to the patient or the patient's caregiver as necessary.

(2) If the patient assessment indicates a need for a discharge plan, the discharge plan must include instructions on post-RNHCI care to be used by the patient or the caregiver in the patient's home, as identified in the discharge plan.

(3) If the RNHCI's patient assessment does not indicate a need for a discharge plan, the beneficiary or his or her legal representative may request a discharge plan. In this case, the RNHCI must develop a discharge plan for the beneficiary.

- 3. Section 403.748 is amended bya. Revising paragraphs (a) introductory text, (a)(4), (b) introductory text, (c) introductory text, (d) introductory text, and (d)(1)(ii); and - b. Adding paragraph (d)(1)(v). 
The revisions and addition read as follows:

\section{\$403.748 Condition of participation: Emergency preparedness.}

(a) Emergency plan. The RNHCI must develop and maintain an emergency preparedness plan that must be reviewed, and updated at least every 2 years. The plan must do all of the following:

(4) Include a process for cooperation and collaboration with local, tribal, regional, State, and Federal emergency preparedness officials' efforts to maintain an integrated response during a disaster or emergency situation.

(b) Policies and procedures. The RNHCI must develop and implement emergency preparedness policies and procedures, based on the emergency plan set forth in paragraph (a) of this section, risk assessment at paragraph (a)(1) of this section, and the communication plan at paragraph (c) of this section. The policies and procedures must be reviewed and updated at least every 2 years. At a minimum, the policies and procedures must address the following:

(c) Communication plan. The RNHCI must develop and maintain an emergency preparedness communication plan that complies with Federal, State, and local laws and must be reviewed and updated at least every 2 years. The communication plan must include all of the following:

(d) Training and testing. The RNHCI must develop and maintain an emergency preparedness training and testing program that is based on the emergency plan set forth in paragraph (a) of this section, risk assessment at paragraph (a)(1) of this section, policies and procedures at paragraph (b) of this section, and the communication plan at paragraph (c) of this section. The training and testing program must be reviewed and updated at least every 2 years.

$$
\text { (1) *** }
$$

(ii) Provide emergency preparedness training at least every 2 years.

(v) If the emergency preparedness policies and procedures are significantly updated, the RNHCI must conduct training on the updated policies and procedures.

\section{PART 416-AMBULATORY SURGICAL SERVICES}

a . The authority citation for part 416 continues to read as follows:

Authority: 42 U.S.C. 273, 1302, 1320b-8, and 1395hh.

- 5. Section 416.41 is amended by revising paragraph (b)(3) to read as follows:

\section{\$416.41 Condition for coverage-} Governing body and management.

* * * * *

(b) ***

(3) The ASC must periodically provide the local hospital with written notice of its operations and patient population served.

- 6. Section 416.47 is amended by revising paragraph (b)(2) to read as follows:

\section{$\S 416.47$ Condition for coverage-Medical} records.

* * * * *

(b) ***

(2) Significant medical history and results of physical examination (as applicable).

7. Section 416.52 is amended by revising paragraph (a) to read as follows:

\$416.52 Condition for coverage-Patient admission, assessment and discharge.

\section{* * * * *}

(a) Standard: Patient assessment and admission. (1) The ASC must develop and maintain a policy that identifies those patients who require a medical history and physical examination prior to surgery. The policy must-

(i) Include the timeframe for medical history and physical examination to be completed prior to surgery.

(ii) Address, but is not limited to, the following factors: Patient age, diagnosis, the type and number of procedures scheduled to be performed on the same surgery date, known comorbidities, and the planned anesthesia level.

(iii) Be based on any applicable nationally recognized standards of practice and guidelines, and any applicable State and local health and safety laws.

(2) Upon admission, each patient must have a pre-surgical assessment completed by a physician who will be performing the surgery or other qualified practitioner in accordance with applicable State health and safety laws, standards of practice, and ASC policy.

(3) The pre-surgical assessment must include documentation of any allergies to drugs and biologicals.
(4) The patient's medical history and physical examination (if any) must be placed in the patient's medical record prior to the surgical procedure.

8. Section 416.54 is amended by-

- a. Revising paragraphs (a)

introductory text, (a)(4), (b) introductory text, (c) introductory text, (d) introductory text, and (d)(1)(ii);

- b. Adding paragraph (d)(1)(v); and

- c. Revising paragraph (d)(2).

The revisions and addition read as follows:

\section{\$416.54 Condition for coverage- Emergency preparedness.}

(a) Emergency plan. The ASC must develop and maintain an emergency preparedness plan that must be reviewed, and updated at least every 2 years. The plan must do the following:

(4) Include a process for cooperation and collaboration with local, tribal, regional, State, and Federal emergency preparedness officials' efforts to maintain an integrated response during a disaster or emergency situation.

(b) Policies and procedures. The ASC must develop and implement emergency preparedness policies and procedures, based on the emergency plan set forth in paragraph (a) of this section, risk assessment at paragraph (a)(1) of this section, and the communication plan at paragraph (c) of this section. The policies and procedures must be reviewed and updated at least every 2 years. At a minimum, the policies and procedures must address the following:

(c) Communication plan. The ASC must develop and maintain an emergency preparedness communication plan that complies with Federal, State, and local laws and must be reviewed and updated at least every 2 years. The communication plan must include all of the following:

(d) Training and testing. The ASC must develop and maintain an emergency preparedness training and testing program that is based on the emergency plan set forth in paragraph (a) of this section, risk assessment at paragraph (a)(1) of this section, policies and procedures at paragraph (b) of this section, and the communication plan at paragraph (c) of this section. The training and testing program must be reviewed and updated at least every 2 years.

$(1) * * *$ 
(ii) Provide emergency preparedness training at least every 2 years.

(v) If the emergency preparedness policies and procedures are significantly updated, the ASC must conduct training on the updated policies and procedures.

(2) Testing. The ASC must conduct exercises to test the emergency plan at least annually. The ASC must do the following:

(i) Participate in a full-scale exercise that is community-based every 2 years; or

(A) When a community-based exercise is not accessible, conduct a facility-based functional exercise every 2 years; or

(B) If the ASC experiences an actual natural or man-made emergency that requires activation of the emergency plan, the ASC is exempt from engaging in its next required community-based or individual, facility-based functional exercise following the onset of the emergency event.

(ii) Conduct an additional exercise at least every 2 years, opposite the year the full-scale or functional exercise under paragraph (d)(2)(i) of this section is conducted, that may include, but is not limited to the following:

(A) A second full-scale exercise that is community-based, or an individual, facility-based functional exercise; or

(B) A mock disaster drill; or

(C) A tabletop exercise or workshop that is led by a facilitator and includes a group discussion using a narrated, clinically-relevant emergency scenario, and a set of problem statements, directed messages, or prepared questions designed to challenge an emergency plan.

(iii) Analyze the ASC's response to and maintain documentation of all drills, tabletop exercises, and emergency events and revise the ASC's emergency plan, as needed.

\section{PART 418-HOSPICE CARE}

9. The authority citation for part 418 is revised to read as follows:

Authority: 42 U.S.C. 1302 and 1395hh.

- 10. Section 418.76 is amended by revising paragraph (a)(1)(iv) to read as follows:

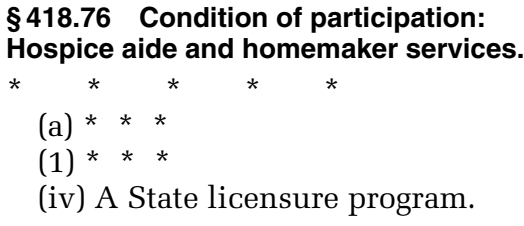

\section{§418.106 [Amended]}

- 11. Section 418.106 is amended by-

- a. Removing paragraph (a)(1);

- b. Redesignating paragraph (a)(2) as paragraph (a)(1); and

c. Adding a new reserved paragraph

(a)(2).

- 12. Section 418.112 is amended by revising paragraph (f) to read as follows:

\$418.112 Condition of participation: Hospices that provide hospice care to residents of a SNF/NF or ICF/IID.

* * * * *

(f) Standard: Orientation and training of staff. Hospice staff, in coordination with SNF/NF or ICF/IID facility staff, must assure orientation of such staff furnishing care to hospice patients in the hospice philosophy, including hospice policies and procedures regarding methods of comfort, pain control, symptom management, as well as principles about death and dying, individual responses to death, patient rights, appropriate forms, and record keeping requirements.

- 13. Section 418.113 is amended by- a. Revising paragraphs (a) introductory text, (a)(4), (b) introductory text, (c) introductory text, (d) introductory text, and (d)(1)(iii);

- b. Adding paragraph (d)(1)(vi);

- c. Revising paragraph (d)(2); and

- d. Adding paragraph (d)(3).

The revisions and additions read as follows:

\section{\$418.113 Condition of participation: Emergency preparedness.}

(a) Emergency plan. The hospice must develop and maintain an emergency preparedness plan that must be reviewed, and updated at least every 2 years. The plan must do the following:

(4) Include a process for cooperation and collaboration with local, tribal, regional, State, or Federal emergency preparedness officials' efforts to maintain an integrated response during a disaster or emergency situation.

(b) Policies and procedures. The hospice must develop and implement emergency preparedness policies and procedures, based on the emergency plan set forth in paragraph (a) of this section, risk assessment at paragraph (a)(1) of this section, and the communication plan at paragraph (c) of this section. The policies and procedures must be reviewed and updated at least every 2 years. At a minimum, the policies and procedures must address the following:

(c) Communication plan. The hospice must develop and maintain an emergency preparedness

communication plan that complies with Federal, State, and local laws and must be reviewed and updated at least every 2 years. The communication plan must include all of the following:

(d) Training and testing. The hospice must develop and maintain an emergency preparedness training and testing program that is based on the emergency plan set forth in paragraph (a) of this section, risk assessment at paragraph (a)(1) of this section, policies and procedures at paragraph (b) of this section, and the communication plan at paragraph (c) of this section. The training and testing program must be reviewed and updated at least every 2 years.

(1) * * *

(iii) Provide emergency preparedness training at least every 2 years.

(vi) If the emergency preparedness policies and procedures are significantly updated, the hospice must conduct training on the updated policies and procedures.

(2) Testing for hospices that provide care in the patient's home. The hospice must conduct exercises to test the emergency plan at least annually. The hospice must do the following:

(i) Participate in a full-scale exercise that is community-based every 2 years; or

(A) When a community-based exercise is not accessible, conduct an individual facility-based functional exercise every 2 years; or

(B) If the hospice experiences a natural or man-made emergency that requires activation of the emergency plan, the hospital is exempt from engaging in its next required full-scale community-based exercise or individual facility-based functional exercise following the onset of the emergency event.

(ii) Conduct an additional exercise every 2 years, opposite the year the fullscale or functional exercise under paragraph (d)(2)(i) of this section is conducted, that may include, but is not limited to the following:

(A) A second full-scale exercise that is community-based or a facility-based functional exercise; or

(B) A mock disaster drill; or

(C) A tabletop exercise or workshop that is led by a facilitator and includes a group discussion using a narrated, clinically-relevant emergency scenario, and a set of problem statements, directed messages, or prepared questions designed to challenge an emergency plan. 
(3) Testing for hospices that provide inpatient care directly. The hospice must conduct exercises to test the emergency plan twice per year. The hospice must do the following:

(i) Participate in an annual full-scale exercise that is community-based; or

(A) When a community-based exercise is not accessible, conduct an annual individual facility-based functional exercise; or

(B) If the hospice experiences a natural or man-made emergency that requires activation of the emergency plan, the hospice is exempt from engaging in its next required full-scale community-based or facility-based functional exercise following the onset of the emergency event.

(ii) Conduct an additional annual exercise that may include, but is not limited to the following:

(A) A second full-scale exercise that is community-based or a facility-based functional exercise; or

(B) A mock disaster drill; or

(C) A tabletop exercise or workshop led by a facilitator that includes a group discussion using a narrated, clinicallyrelevant emergency scenario, and a set of problem statements, directed messages, or prepared questions designed to challenge an emergency plan.

(iii) Analyze the hospice's response to and maintain documentation of all drills, tabletop exercises, and emergency events and revise the hospice's emergency plan, as needed.

\section{PART 441-SERVICES: REQUIREMENTS AND LIMITS APPLICABLE TO SPECIFIC SERVICES}

14. The authority citation for part 441 is revised to read as follows:

\section{Authority: 42 U.S.C. 1302.}

15. Section 441.184 is amended bya. Revising paragraphs (a) introductory text, (a)(4), (b) introductory text, (c) introductory text, (d) introductory text, and (d)(1)(ii);

- b. Adding paragraph (d)(1)(v); and

- c. Revising paragraph (d)(2).

The revisions and addition read as follows:

\section{\$441.184 Emergency preparedness.}

(a) Emergency plan. The PRTF must develop and maintain an emergency preparedness plan that must be reviewed, and updated at least every 2 years. The plan must do the following:

(4) Include a process for cooperation and collaboration with local, tribal, regional, State, and Federal emergency preparedness officials' efforts to maintain an integrated response during a disaster or emergency situation.

(b) Policies and procedures. The PRTF must develop and implement emergency preparedness policies and procedures, based on the emergency plan set forth in paragraph (a) of this section, risk assessment at paragraph (a)(1) of this section, and the communication plan at paragraph (c) of this section. The policies and procedures must be reviewed and updated at least every 2 years. At a minimum, the policies and procedures must address the following:

(c) Communication plan. The PRTF must develop and maintain an emergency preparedness communication plan that complies with Federal, State, and local laws and must be reviewed and updated at least every 2 years. The communication plan must include all of the following:

(d) Training and testing. The PRTF must develop and maintain an emergency preparedness training program that is based on the emergency plan set forth in paragraph (a) of this section, risk assessment at paragraph (a)(1) of this section, policies and procedures at paragraph (b) of this section, and the communication plan at paragraph (c) of this section. The training and testing program must be reviewed and updated at least every 2 years.

(1) * * *

(ii) After initial training, provide emergency preparedness training every 2 years.

(v) If the emergency preparedness policies and procedures are significantly updated, the PRTF must conduct training on the updated policies and procedures.

(2) Testing. The PRTF must conduct exercises to test the emergency plan twice per year. The PRTF must do the following:

(i) Participate in an annual full-scale exercise that is community-based; or

(A) When a community-based exercise is not accessible, conduct an annual individual, facility-based functional exercise; or

(B) If the PRTF experiences an actual natural or man-made emergency that requires activation of the emergency plan, the PRTF is exempt from engaging in its next required full-scale community-based or individual, facilitybased functional exercise following the onset of the emergency event. (ii) Conduct an additional annual exercise that may include, but is not limited to the following:

(A) A second full-scale exercise that is community-based or individual, a facility-based functional exercise; or

(B) A mock disaster drill; or

(C) A tabletop exercise or workshop that is led by a facilitator and includes a group discussion, using a narrated, clinically-relevant emergency scenario, and a set of problem statements, directed messages, or prepared questions designed to challenge an emergency plan.

(iii) Analyze the PRTF's response to and maintain documentation of all drills, tabletop exercises, and emergency events and revise the PRTF's emergency plan, as needed.

\section{PART 460-PROGRAMS OF ALL- INCLUSIVE CARE FOR THE ELDERLY (PACE)}

- 16. The authority citation for part 460 is revised to read as follows:

Authority: 42 U.S.C. 1302, 1395, 1395eee(f), and 1396u-4(f).

- 17. Section 460.84 is amended by- a. Revising paragraphs (a) introductory text, (a)(4), (b) introductory text, (c) introductory text, (d) introductory text, and (d)(1)(ii);

- b. Adding paragraph (d)(1)(v); and

- c. Revising paragraph (d)(2).

The revisions and addition read as follows:

\section{\$460.84 Emergency preparedness.}

(a) Emergency plan. The PACE organization must develop and maintain an emergency preparedness plan that must be reviewed, and updated at least every 2 years. The plan must do the following:

(4) Include a process for cooperation and collaboration with local, tribal, regional, State, and Federal emergency preparedness officials' efforts to maintain an integrated response during a disaster or emergency situation.

(b) Policies and procedures. The PACE organization must develop and implement emergency preparedness policies and procedures, based on the emergency plan set forth in paragraph (a) of this section, risk assessment at paragraph (a)(1) of this section, and the communication plan at paragraph (c) of this section. The policies and procedures must address management of medical and nonmedical emergencies, including, but not limited to: Fire; equipment, power, or water 
failure; care-related emergencies; and natural disasters likely to threaten the health or safety of the participants, staff, or the public. Policies and procedures must be reviewed and updated at least every 2 years. At a minimum, the policies and procedures must address the following:

(c) Communication plan. The PACE organization must develop and maintain an emergency preparedness communication plan that complies with Federal, State, and local laws and must be reviewed and updated at least every 2 years. The communication plan must include all of the following:

(d) Training and testing. The PACE organization must develop and maintain an emergency preparedness training and testing program that is based on the emergency plan set forth in paragraph (a) of this section, risk assessment at paragraph (a)(1) of this section, policies and procedures at paragraph (b) of this section, and the communication plan at paragraph (c) of this section. The training and testing program must be reviewed and updated at least every 2 years.

(1) ***

(ii) Provide emergency preparedness training at least every 2 years.

(v) If the emergency preparedness policies and procedures are significantly updated, the PACE must conduct training on the updated policies and procedures.

(2) Testing. The PACE organization must conduct exercises to test the emergency plan at least annually. The PACE organization must do the following:

(i) Participate in a full-scale exercise that is community-based or;

(A) When a community-based exercise is not accessible, conduct an annual individual, facility-based functional exercise every 2 years; or

(B) If the PACE experiences an actual natural or man-made emergency that requires activation of the emergency plan, the PACE is exempt from engaging in its next required full-scale community-based or individual, facilitybased functional exercise following the onset of the emergency event.

(ii) Conduct an additional exercise every 2 years opposite the year the fullscale or functional exercise under paragraph (d)(2)(i) of this section is conducted that may include, but is not limited to the following:

(A) A second full-scale exercise that is community-based or an individual, facility-based functional exercise; or
(B) A mock disaster drill; or

(C) A tabletop exercise or workshop that is led by a facilitator and includes a group discussion, using a narrated, clinically-relevant emergency scenario, and a set of problem statements, directed messages, or prepared questions designed to challenge an emergency plan.

(iii) Analyze the PACE's response to and maintain documentation of all drills, tabletop exercises, and emergency events and revise the PACE's emergency plan, as needed.

\section{PART 482-CONDITIONS OF PARTICIPATION FOR HOSPITALS}

- 18. The authority citation for part 482 is revised to read as follows:

Authority: 42 U.S.C. 1302, 1395hh, and 1395rr, unless otherwise noted.

19. Section 482.13 is amended by revising paragraphs (e)(5), (e)(8)(ii), (e)(10) and (11), (e)(12)(i), (e)(14), and (g)(4)(ii) to read as follows:

\section{\$482.13 Condition of participation:} Patient's rights.

* * * * *

(e) ***

(5) The use of restraint or seclusion must be in accordance with the order of a physician or other licensed practitioner who is responsible for the care of the patient and authorized to order restraint or seclusion by hospital policy in accordance with State law.

\section{(8) ***}

(ii) After 24 hours, before writing a new order for the use of restraint or seclusion for the management of violent or self-destructive behavior, a physician or other licensed practitioner who is responsible for the care of the patient and authorized to order restraint or seclusion by hospital policy in accordance with State law must see and assess the patient.

(10) The condition of the patient who is restrained or secluded must be monitored by a physician, other licensed practitioner, or trained staff that have completed the training criteria specified in paragraph (f) of this section at an interval determined by hospital policy.

(11) Physician and other licensed practitioner training requirements must be specified in hospital policy. At a minimum, physicians and other licensed practitioners authorized to order restraint or seclusion by hospital policy in accordance with State law must have a working knowledge of hospital policy regarding the use of restraint or seclusion.

(12) * * *

(i) By a-

(A) Physician or other licensed practitioner.

(B) Registered nurse who has been trained in accordance with the requirements specified in paragraph (f) of this section.

(14) If the face-to-face evaluation specified in paragraph (e)(12) of this section is conducted by a trained registered nurse, the trained registered nurse must consult the attending physician or other licensed practitioner who is responsible for the care of the patient as soon as possible after the completion of the 1-hour face-to-face evaluation.

* * * * *

$(\mathrm{g}) * * *$

(4) ***

(ii) Each entry must document the patient's name, date of birth, date of death, name of attending physician or other licensed practitioner who is responsible for the care of the patient, medical record number, and primary diagnosis(es).

- 20. Section 482.15 is amendeda. By revising paragraphs (a) introductory text, (a)(4), (b) introductory text, (c) introductory text, (d) introductory text, and (d)(1)(ii); - b. By adding paragraph (d)(1)(v); c. By revising paragraph (d)(2); - d. In paragraph (g) introductory text, by removing the phrase "transplant centers" and adding into its place the phrase "transplant programs"; and - e. In paragraphs (g)(1) and (2), by removing the phrase "transplant center" and adding into its place the phrase “transplant program”.

The revisions and addition read as follows:

\section{\$482.15 Condition of participation: Emergency preparedness.}

(a) Emergency plan. The hospital must develop and maintain an emergency preparedness plan that must be reviewed, and updated at least every 2 years. The plan must do the following:

(4) Include a process for cooperation and collaboration with local, tribal, regional, State, and Federal emergency preparedness officials' efforts to maintain an integrated response during a disaster or emergency situation.

(b) Policies and procedures. The hospital must develop and implement emergency preparedness policies and 
procedures, based on the emergency plan set forth in paragraph (a) of this section, risk assessment at paragraph (a)(1) of this section, and the communication plan at paragraph (c) of this section. The policies and procedures must be reviewed and updated at least every 2 years. At a minimum, the policies and procedures must address the following:

(c) Communication plan. The hospital must develop and maintain an emergency preparedness communication plan that complies with Federal, State, and local laws and must be reviewed and updated at least every 2 years. The communication plan must include all of the following:

(d) Training and testing. The hospital must develop and maintain an emergency preparedness training and testing program that is based on the emergency plan set forth in paragraph (a) of this section, risk assessment at paragraph (a)(1) of this section, policies and procedures at paragraph (b) of this section, and the communication plan at paragraph (c) of this section. The training and testing program must be reviewed and updated at least every 2 years.

(1) * * *

(ii) Provide emergency preparedness training at least every 2 years.

(v) If the emergency preparedness policies and procedures are significantly updated, the hospital must conduct training on the updated policies and procedures.

(2) Testing. The hospital must conduct exercises to test the emergency plan at least twice per year. The hospital must do all of the following:

(i) Participate in an annual full-scale exercise that is community-based; or

(A) When a community-based exercise is not accessible, conduct an annual individual, facility-based functional exercise; or.

(B) If the hospital experiences an actual natural or man-made emergency that requires activation of the emergency plan, the hospital is exempt from engaging in its next required fullscale community-based exercise or individual, facility-based functional exercise following the onset of the emergency event.

(ii) Conduct an additional annual exercise that may include, but is not limited to the following:

(A) A second full-scale exercise that is community-based or an individual, facility-based functional exercise; or

(B) A mock disaster drill; or
(C) A tabletop exercise or workshop that is led by a facilitator and includes a group discussion, using a narrated, clinically-relevant emergency scenario, and a set of problem statements, directed messages, or prepared questions designed to challenge an emergency plan.

(iii) Analyze the hospital's response to and maintain documentation of all drills, tabletop exercises, and emergency events, and revise the hospital's emergency plan, as needed.

21. Section 482.21 is amended by revising paragraph (b)(1) and adding paragraph (f) to read as follows:

§482.21 Condition of participation: Quality assessment and performance improvement program.

(b) ***

(1) The program must incorporate quality indicator data including patient care data, and other relevant data such as data submitted to or received from Medicare quality reporting and quality performance programs, including but not limited to data related to hospital readmissions and hospital-acquired conditions.

(f) Standard: Unified and integrated QAPI program for multi-hospital systems. If a hospital is part of a hospital system consisting of multiple separately certified hospitals using a system governing body that is legally responsible for the conduct of two or more hospitals, the system governing body can elect to have a unified and integrated QAPI program for all of its member hospitals after determining that such a decision is in accordance with all applicable State and local laws. The system governing body is responsible and accountable for ensuring that each of its separately certified hospitals meets all of the requirements of this section. Each separately certified hospital subject to the system governing body must demonstrate that:

(1) The unified and integrated QAPI program is established in a manner that takes into account each member hospital's unique circumstances and any significant differences in patient populations and services offered in each hospital; and

(2) The unified and integrated QAPI program establishes and implements policies and procedures to ensure that the needs and concerns of each of its separately certified hospitals, regardless of practice or location, are given due consideration, and that the unified and integrated QAPI program has mechanisms in place to ensure that issues localized to particular hospitals are duly considered and addressed.

- 22. Section 482.22 is amended by- a. Revising paragraphs (c)(5)(i) and (ii);

- b. Adding paragraphs (c)(5)(iii), (iv), and (v); and

a c. Removing paragraph (d).

The revisions and additions read as follows:

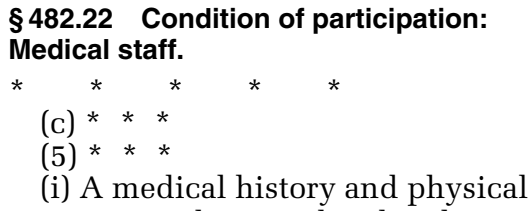

examination be completed and documented for each patient no more than 30 days before or 24 hours after admission or registration, but prior to surgery or a procedure requiring anesthesia services, and except as provided under paragraph (c)(5)(iii) of this section. The medical history and physical examination must be completed and documented by a physician (as defined in section 1861(r) of the Act), an oral and maxillofacial surgeon, or other qualified licensed individual in accordance with State law and hospital policy.

(ii) An updated examination of the patient, including any changes in the patient's condition, be completed and documented within 24 hours after admission or registration, but prior to surgery or a procedure requiring anesthesia services, when the medical history and physical examination are completed within 30 days before admission or registration, and except as provided under paragraph (c)(5)(iii) of this section. The updated examination of the patient, including any changes in the patient's condition, must be completed and documented by a physician (as defined in section 1861(r) of the Act), an oral and maxillofacial surgeon, or other qualified licensed individual in accordance with State law and hospital policy.

(iii) An assessment of the patient (in lieu of the requirements of paragraphs (c)(5)(i) and (ii) of this section) be completed and documented after registration, but prior to surgery or a procedure requiring anesthesia services, when the patient is receiving specific outpatient surgical or procedural services and when the medical staff has chosen to develop and maintain a policy that identifies, in accordance with the requirements at paragraph (c)(5)(v) of this section, specific patients as not requiring a comprehensive medical history and physical examination, or any update to it, prior to specific 
outpatient surgical or procedural services. The assessment must be completed and documented by a physician (as defined in section 1861(r) of the Act), an oral and maxillofacial surgeon, or other qualified licensed individual in accordance with State law and hospital policy.

(iv) The medical staff develop and maintain a policy that identifies those patients for whom the assessment requirements of paragraph (c)(5)(iii) of this section would apply. The provisions of paragraphs (c)(5)(iii), (iv), and $(\mathrm{v})$ of this section do not apply to a medical staff that chooses to maintain a policy that adheres to the requirements of paragraphs of (c)(5)(i) and (ii) of this section for all patients.

(v) The medical staff, if it chooses to develop and maintain a policy for the identification of specific patients to whom the assessment requirements in paragraph (c)(5)(iii) of this section would apply, must demonstrate evidence that the policy applies only to those patients receiving specific outpatient surgical or procedural services as well as evidence that the policy is based on:

(A) Patient age, diagnoses, the type and number of surgeries and procedures scheduled to be performed, comorbidities, and the level of anesthesia required for the surgery or procedure.

(B) Nationally recognized guidelines and standards of practice for assessment of specific types of patients prior to specific outpatient surgeries and procedures.

(C) Applicable state and local health and safety laws.

23. Section 482.23 is amended by a. Revising paragraphs (b) introductory text and (b)(4) and (6);

- b. Adding paragraph (b)(7); and

c. Revising (c)(1) introductory text and (c)(3).

The revisions and addition read as follows:

\section{$\S 482.23$ Condition of participation: Nursing services.}

(b) Standard: Staffing and delivery of care. The nursing service must have adequate numbers of licensed registered nurses, licensed practical (vocational) nurses, and other personnel to provide nursing care to all patients as needed. There must be supervisory and staff personnel for each department or nursing unit to ensure, when needed, the immediate availability of a registered nurse for the care of any patient.
(4) The hospital must ensure that the nursing staff develops and keeps current a nursing care plan for each patient that reflects the patient's goals and the nursing care to be provided to meet the patient's needs. The nursing care plan may be part of an interdisciplinary care plan.

(6) All licensed nurses who provide services in the hospital must adhere to the policies and procedures of the hospital. The director of nursing service must provide for the adequate supervision and evaluation of the clinical activities of all nursing personnel which occur within the responsibility of the nursing service, regardless of the mechanism through which those personnel are providing services (that is, hospital employee, contract, lease, other agreement, or volunteer).

(7) The hospital must have policies and procedures in place establishing which outpatient departments, if any, are not required under hospital policy to have a registered nurse present. The policies and procedures must:

(i) Establish the criteria such outpatient departments must meet, taking into account the types of services delivered, the general level of acuity of patients served by the department, and the established standards of practice for the services delivered;

(ii) Establish alternative staffing plans;

(iii) Be approved by the director of nursing;

(iv) Be reviewed at least once every 3 years.

(c) * * *

(1) Drugs and biologicals must be prepared and administered in accordance with Federal and State laws, the orders of the practitioner or practitioners responsible for the patient's care, and accepted standards of practice.

(3) With the exception of influenza and pneumococcal vaccines, which may be administered per physician-approved hospital policy after an assessment of contraindications, orders for drugs and biologicals must be documented and signed by a practitioner who is authorized to write orders in accordance with State law and hospital policy, and who is responsible for the care of the patient.

(i) If verbal orders are used, they are to be used infrequently.

(ii) When verbal orders are used, they must only be accepted by persons who are authorized to do so by hospital policy and procedures consistent with Federal and State law. (iii) Orders for drugs and biologicals may be documented and signed by other practitioners only if such practitioners are acting in accordance with State law, including scope-of-practice laws, hospital policies, and medical staff bylaws, rules, and regulations.

24. Section 482.24 is amended by revising paragraphs (c)(4)(i)(A) and (B), and adding paragraph (C)(4)(i)(C) to read as follows:

$\$ 482.24$ Condition of participation:
Medical record services.
$* * *$
(c) *** $* *$
(4) ***
(i) ***

(A) A medical history and physical examination completed and documented no more than 30 days before or 24 hours after admission or registration, but prior to surgery or a procedure requiring anesthesia services, and except as provided under paragraph (c)(4)(i)(C) of this section. The medical history and physical examination must be placed in the patient's medical record within 24 hours after admission or registration, but prior to surgery or a procedure requiring anesthesia services.

(B) An updated examination of the patient, including any changes in the patient's condition, when the medical history and physical examination are completed within 30 days before admission or registration, and except as provided under paragraph (c)(4)(i)(C) of this section. Documentation of the updated examination must be placed in the patient's medical record within 24 hours after admission or registration, but prior to surgery or a procedure requiring anesthesia services.

(C) An assessment of the patient (in lieu of the requirements of paragraphs (c)(4)(i)(A) and (B) of this section) completed and documented after registration, but prior to surgery or a procedure requiring anesthesia services, when the patient is receiving specific outpatient surgical or procedural services and when the medical staff has chosen to develop and maintain a policy that identifies, in accordance with the requirements at $\S 482.22(\mathrm{c})(5)(\mathrm{v})$, specific patients as not requiring a comprehensive medical history and physical examination, or any update to it, prior to specific outpatient surgical or procedural services.

25. Section 482.27 is amended by revising paragraph (b)(7) and removing paragraph (b)(11).

The revision reads as follows: 
\$482.27 Condition of participation: Laboratory services.

* (b) * * *

(7) Timeframe for notification- For donors tested on or after February 20, 2008. For notifications resulting from donors tested on or after February 20, 2008 as set forth at 21 CFR 610.46 and 610.47 the notification effort begins when the blood collecting establishment notifies the hospital that it received potentially HIV or HCV infectious blood and blood components. The hospital must make reasonable attempts to give notification over a period of 12 weeks unless-

(i) The patient is located and notified; or

(ii) The hospital is unable to locate the patient and documents in the patient's medical record the extenuating circumstances beyond the hospital's control that caused the notification timeframe to exceed 12 weeks.

26. Section 482.42 is revised to read as follows:

\section{\$482.42 Condition of participation: Infection prevention and control and antibiotic stewardship programs.}

The hospital must have active hospital-wide programs for the surveillance, prevention, and control of HAIs and other infectious diseases, and for the optimization of antibiotic use through stewardship. The programs must demonstrate adherence to nationally recognized infection prevention and control guidelines, as well as to best practices for improving antibiotic use where applicable, and for reducing the development and transmission of HAIs and antibioticresistant organisms. Infection prevention and control problems and antibiotic use issues identified in the programs must be addressed in collaboration with the hospital-wide quality assessment and performance improvement (QAPI) program.

(a) Standard: Infection prevention and control program organization and policies. The hospital must demonstrate that:

(1) An individual (or individuals), who is qualified through education, training, experience, or certification in infection prevention and control, is appointed by the governing body as the infection preventionist(s)/infection control professional(s) responsible for the infection prevention and control program and that the appointment is based on the recommendations of medical staff leadership and nursing leadership;
(2) The hospital infection prevention and control program, as documented in its policies and procedures, employs methods for preventing and controlling the transmission of infections within the hospital and between the hospital and other institutions and settings;

(3) The infection prevention and control program includes surveillance, prevention, and control of HAIs, including maintaining a clean and sanitary environment to avoid sources and transmission of infection, and addresses any infection control issues identified by public health authorities; and

(4) The infection prevention and control program reflects the scope and complexity of the hospital services provided.

(b) Standard: Antibiotic stewardship program organization and policies. The hospital must demonstrate that:

(1) An individual (or individuals), who is qualified through education, training, or experience in infectious diseases and/or antibiotic stewardship, is appointed by the governing body as the leader(s) of the antibiotic stewardship program and that the appointment is based on the recommendations of medical staff leadership and pharmacy leadership;

(2) The hospital-wide antibiotic stewardship program:

(i) Demonstrates coordination among all components of the hospital responsible for antibiotic use and resistance, including, but not limited to, the infection prevention and control program, the QAPI program, the medical staff, nursing services, and pharmacy services;

(ii) Documents the evidence-based use of antibiotics in all departments and services of the hospital; and

(iii) Documents any improvements, including sustained improvements, in proper antibiotic use;

(3) The antibiotic stewardship program adheres to nationally recognized guidelines, as well as best practices, for improving antibiotic use; and

(4) The antibiotic stewardship program reflects the scope and complexity of the hospital services provided.

(c) Standard: Leadership responsibilities. (1) The governing body must ensure all of the following:

(i) Systems are in place and operational for the tracking of all infection surveillance, prevention, and control, and antibiotic use activities, in order to demonstrate the implementation, success, and sustainability of such activities. (ii) All HAIs and other infectious diseases identified by the infection prevention and control program as well as antibiotic use issues identified by the antibiotic stewardship program are addressed in collaboration with hospital QAPI leadership.

(2) The infection preventionist(s)/ infection control professional(s) is responsible for:

(i) The development and implementation of hospital-wide infection surveillance, prevention, and control policies and procedures that adhere to nationally recognized guidelines.

(ii) All documentation, written or electronic, of the infection prevention and control program and its surveillance, prevention, and control activities.

(iii) Communication and collaboration with the hospital's QAPI program on infection prevention and control issues.

(iv) Competency-based training and education of hospital personnel and staff, including medical staff, and, as applicable, personnel providing contracted services in the hospital, on the practical applications of infection prevention and control guidelines, policies, and procedures.

(v) The prevention and control of HAIs, including auditing of adherence to infection prevention and control policies and procedures by hospital personnel.

(vi) Communication and collaboration with the antibiotic stewardship program.

(3) The leader(s) of the antibiotic stewardship program is responsible for:

(i) The development and implementation of a hospital-wide antibiotic stewardship program, based on nationally recognized guidelines, to monitor and improve the use of antibiotics.

(ii) All documentation, written or electronic, of antibiotic stewardship program activities.

(iii) Communication and collaboration with medical staff, nursing, and pharmacy leadership, as well as with the hospital's infection prevention and control and QAPI programs, on antibiotic use issues.

(iv) Competency-based training and education of hospital personnel and staff, including medical staff, and, as applicable, personnel providing contracted services in the hospital, on the practical applications of antibiotic stewardship guidelines, policies, and procedures.

(d) Standard: Unified and integrated infection prevention and control and antibiotic stewardship programs for multi-hospital systems. If a hospital is 
part of a hospital system consisting of multiple separately certified hospitals using a system governing body that is legally responsible for the conduct of two or more hospitals, the system governing body can elect to have unified and integrated infection prevention and control and antibiotic stewardship programs for all of its member hospitals after determining that such a decision is in accordance with all applicable State and local laws. The system governing body is responsible and accountable for ensuring that each of its separately certified hospitals meets all of the requirements of this section. Each separately certified hospital subject to the system governing body must demonstrate that:

(1) The unified and integrated infection prevention and control and antibiotic stewardship programs are established in a manner that takes into account each member hospital's unique circumstances and any significant differences in patient populations and services offered in each hospital;

(2) The unified and integrated infection prevention and control and antibiotic stewardship programs establish and implement policies and procedures to ensure that the needs and concerns of each of its separately certified hospitals, regardless of practice or location, are given due consideration;

(3) The unified and integrated infection prevention and control and antibiotic stewardship programs have mechanisms in place to ensure that issues localized to particular hospitals are duly considered and addressed; and

(4) A qualified individual (or individuals) with expertise in infection prevention and control and in antibiotic stewardship has been designated at the hospital as responsible for communicating with the unified infection prevention and control and antibiotic stewardship programs, for implementing and maintaining the policies and procedures governing infection prevention and control and antibiotic stewardship as directed by the unified infection prevention and control and antibiotic stewardship programs, and for providing education and training on the practical applications of infection prevention and control and antibiotic stewardship to hospital staff.

- 27. Section 482.51 is amended by revising paragraphs (b)(1)(i) and (ii) and adding paragraph (b)(1)(iii) to read as follows:

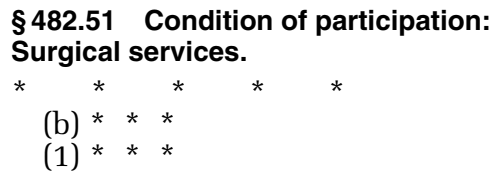

(i) A medical history and physical examination must be completed and documented no more than 30 days before or 24 hours after admission or registration, and except as provided under paragraph (b)(1)(iii) of this section.

(ii) An updated examination of the patient, including any changes in the patient's condition, must be completed and documented within 24 hours after admission or registration when the medical history and physical examination are completed within 30 days before admission or registration, and except as provided under paragraph (b)(1)(iii) of this section.

(iii) An assessment of the patient must be completed and documented after registration (in lieu of the requirements of paragraphs (b)(1)(i) and (ii) of this section) when the patient is receiving specific outpatient surgical or procedural services and when the medical staff has chosen to develop and maintain a policy that identifies, in accordance with the requirements at $\S 482.22(\mathrm{c})(5)(\mathrm{v})$, specific patients as not requiring a comprehensive medical history and physical examination, or any update to it, prior to specific outpatient surgical or procedural services.

* * * * *

- 28. Section 482.58 is amended by-

- a. Revising paragraph (b)(1);

- b. Removing paragraph (b)(4);

- c. Redesignating paragraphs (b)(5)

through (8) as paragraphs (b)(4) through (7); and

- d. Revising newly redesignated paragraphs (b)(4), (5), and (7).

The revisions read as follows:

§482.58 Special requirements for hospital providers of long-term care services ("swing-beds").

* * *

(b) ***

(1) Resident rights (§ 483.10(b)(7), (c)(1), (c)(2)(iii), (c)(6), (d), (e)(2) and (4), (f)(4)(ii) and (iii), (h), (g)(8) and (17), and (g)(18) introductory text of this chapter).

(4) Social services (§ $483.40(d)$ of this chapter).

(5) Discharge summary (§ 483.20(l)).

(7) Dental services (§ 483.55(a)(2), (3), (4), and (5) and (b) of this chapter).

- 29. Section 482.61 is amended by revising paragraph (d) to read as follows:

§482.61 Condition of participation: Special medical record requirements for psychiatric hospitals. (d) Standard: Recording progress. Progress notes must be recorded by the physicians(s), psychologists, or other licensed independent practitioner(s) responsible for the care of the patient as specified in § 482.12(c); nurse, social worker and, when appropriate, others significantly involved in active treatment modalities. The frequency of progress notes is determined by the condition of the patient but must be recorded at least weekly for the first 2 months and at least once a month thereafter and must contain recommendations for revisions in the treatment plan as indicated as well as precise assessment of the patient's progress in accordance with the original or revised treatment plan.

\section{§482.68 [Amended]}

- 30. Section 482.68 is amended- a. In the section heading by removing the phrase "transplant centers" and adding in its place the phrase "transplant programs"; and

a. In the introductory text and in paragraph (b) by removing the phrase "transplant center" and adding in its place the phrase "transplant program".

- 31. Section 482.70 is amended-

a. In the definition of "Adverse event" by removing the phrase "transplant centers" and adding in its place the phrase "transplant programs";

- b. By removing the definition of

"Heart-Lung transplant center";

- c. By adding definitions for "Heart-

Lung transplant program” and

"Intestine transplant program" in

alphabetical order;

- d. By removing the definition of

"Intestine transplant center";

- e. By adding a definition for "Intestine transplant program” in alphabetical order;

- $\mathrm{f}$. By removing the definition of

"Pancreas transplant center";

- g. By adding a definition for

"Pancreas transplant program" in

alphabetical order;

- h. By removing the definition of

“Transplant center"; and

- i. By revising the definition of

“'Transplant program”.

The additions and revision read as follows:

\section{\$482.70 Definitions.}

Heart-Lung transplant program means a transplant program that is located in a hospital with an existing Medicareapproved heart transplant program and an existing Medicare-approved lung program that performs combined heartlung transplants. 
Intestine transplant program means a Medicare-approved liver transplant program that performs intestine transplants, combined liver-intestine transplants, or multivisceral transplants.

Pancreas transplant program means a Medicare-approved kidney transplant program that performs pancreas transplants alone or subsequent to a kidney transplant as well as kidneypancreas transplants.

Transplant program means an organspecific transplant program within a transplant hospital (as defined in this section).

\section{$\S \S 482.72,482.74,482.78$, and 482.80 [Amended]}

32. In the following table, for each section and paragraph indicated in the first two columns, remove the phrase indicated in the third column each time it appears and add the reference indicated in the fourth column:

\begin{tabular}{|c|c|c|c|}
\hline Section & Paragraphs & Remove & Add \\
\hline 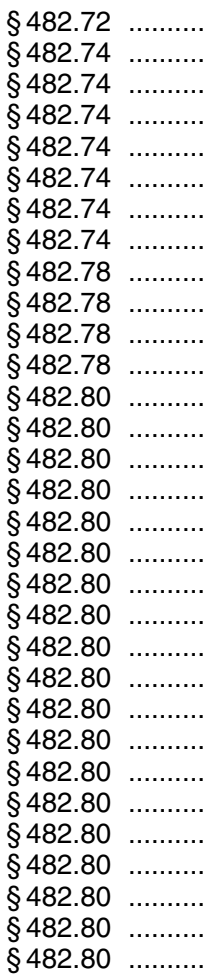 & (a) introductory text (a) introductory text (a)(1) & $\begin{array}{l}\text { transplant center } \\
\text { transplant center } \\
\text { center's } \\
\text { transplant center } \\
\text { center's } \\
\text { transplant center } \\
\text { transplant center } \\
\text { transplant center } \\
\text { transplant centers } \\
\text { transplant center } \\
\text { transplant center } \\
\text { transplant center } \\
\text { transplant centers } \\
\text { transplant centers } \\
\text { transplant center } \\
\text { beneficiary } \\
\text { transplant center } \\
\text { center } \\
\text { transplant center's } \\
\text { center-specific report } \\
\text { beneficiaries } \\
\text { center's } \\
\text { transplant center } \\
\text { center } \\
\text { transplant center } \\
\text { center } \\
\text { transplant center } \\
\text { center } \\
\text { transplant center } \\
\text { center } \\
\text { transplant center }\end{array}$ & $\begin{array}{l}\text { transplant program. } \\
\text { transplant program. } \\
\text { hospital's. } \\
\text { transplant program. } \\
\text { program's. } \\
\text { transplant program. } \\
\text { transplant program. } \\
\text { transplant program. } \\
\text { transplant programs. } \\
\text { transplant program. } \\
\text { transplant program. } \\
\text { transplant program. } \\
\text { transplant programs. } \\
\text { transplant programs. } \\
\text { transplant program. } \\
\text { recipient. } \\
\text { transplant program. } \\
\text { program. } \\
\text { transplant program's. } \\
\text { program-specific report. } \\
\text { recipients. } \\
\text { program's. } \\
\text { transplant program. } \\
\text { program. } \\
\text { transplant program. } \\
\text { program. } \\
\text { transplant program. } \\
\text { program. } \\
\text { transplant program. } \\
\text { program. } \\
\text { transplant program. }\end{array}$ \\
\hline
\end{tabular}

\section{\$482.82 [Removed]}

- 33. Section 482.82 is removed.

34. The undesignated center heading preceding $\S 482.90$ is revised to read

"Transplant Program Process Requirements".
$\S \S 482.90,482.92,482.94,482.96,482.98$, 482.100, and 482.102 [Amended]

35. In the following table, for each section and paragraph indicated in the first two columns, remove the phrase indicated in the third column each time

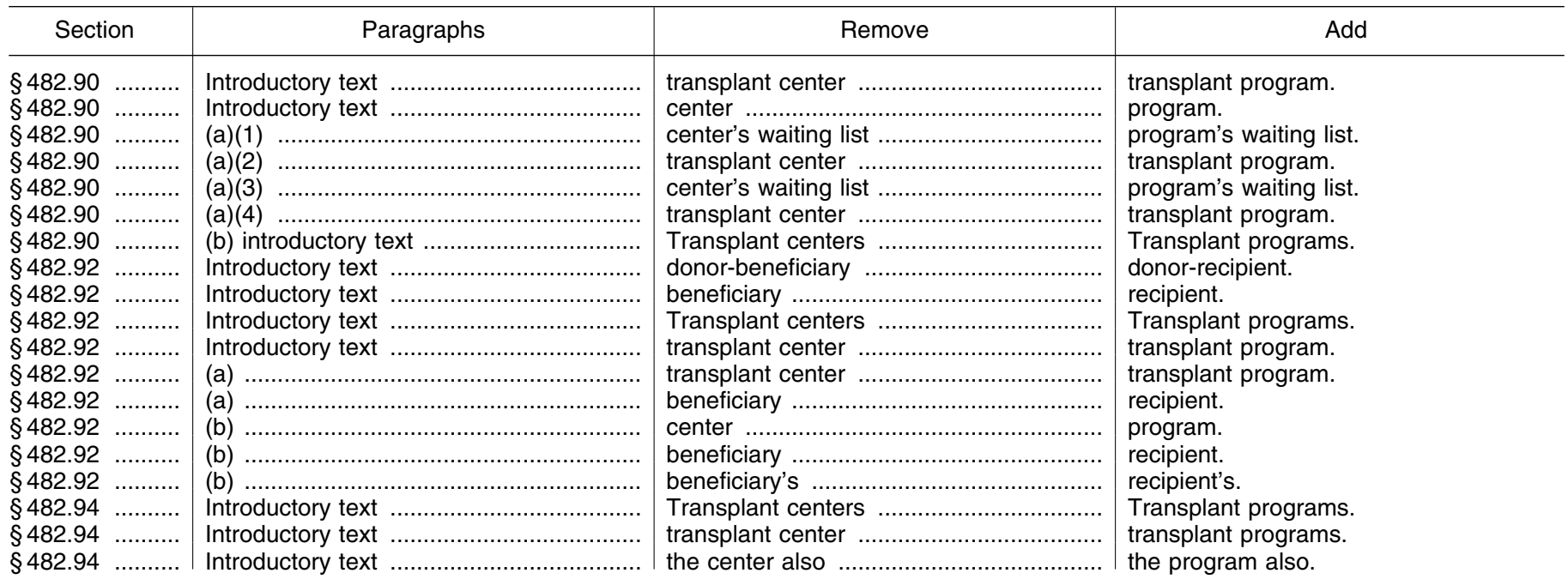

it appears and add the reference indicated in the fourth column: 
Federal Register/Vol. 84, No. 189/Monday, September 30, 2019/Rules and Regulations

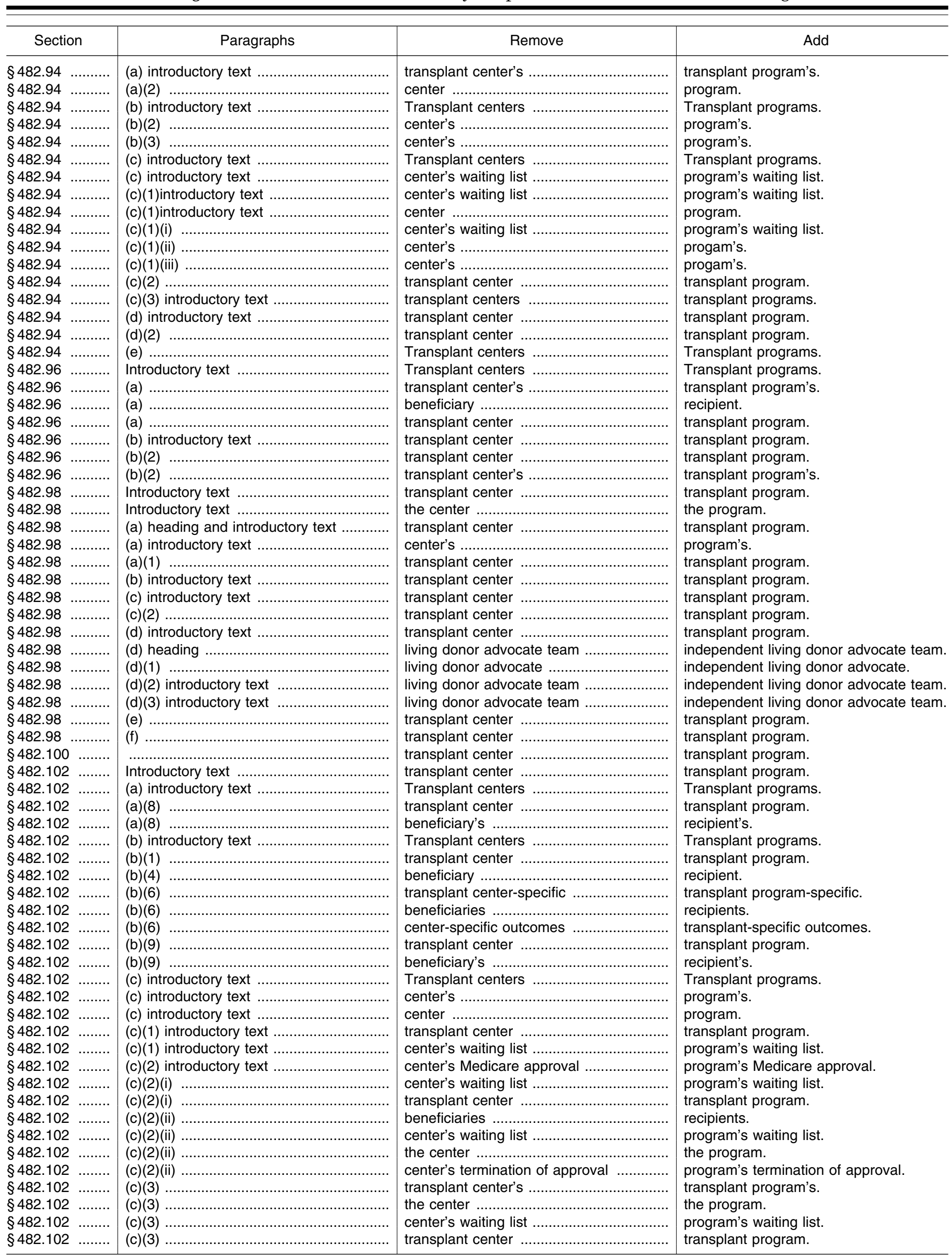


36. Section 482.102 is further amended by revising paragraph (a)(5) to read as follows:

§482.102 Condition of participation: Patient and living donor rights.

(a) ***
(5) National and transplant programspecific outcomes, from the most recent SRTR program-specific report, including (but not limited to) the transplant program's observed and expected 1-year patient and graft survival, and national 1-year patient and graft survival;

\section{\$482.104 [Amended]}

- 37. For $\S 482.104$, in the following table, for the heading and each paragraph indicated in the first column, remove the phrase indicated in the second column each time it appears and add the reference indicated in the third column:

\begin{tabular}{|c|c|}
\hline Paragraphs & Remove \\
\hline $\begin{array}{l}\text { Section heading } \\
\text { (a) } \\
\text { (a) } \\
\text { (b) } \\
\text { (c) }\end{array}$ & 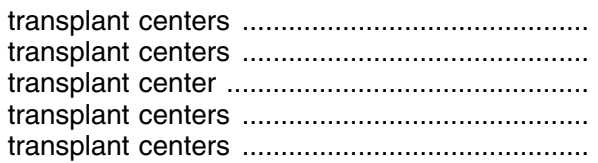 \\
\hline
\end{tabular}

transplant programs. transplant programs. transplant program. transplant programs. transplant programs.

\section{PART 483-REQUIREMENTS FOR STATES AND LONG TERM CARE FACILITIES}

- 38. The authority citation for part 483 is revised to read as follows:

Authority: 42 U.S.C. 1302, 1320a-7, 1395i, 1395hh and 1396r.

39. Section 483.73 is amended by revising paragraphs $(\mathrm{a})(4)$ and $(\mathrm{d})(2)$ to read as follows:

\section{\$ 483.73 Emergency preparedness.}

(a) * * *

(4) Include a process for cooperation and collaboration with local, tribal, regional, State, or Federal emergency preparedness officials' efforts to maintain an integrated response during a disaster or emergency situation.

\section{(d)***}

(2) Testing. The LTC facility must conduct exercises to test the emergency plan at least twice per year, including unannounced staff drills using the emergency procedures. The LTC facility must do the following:

(i) Participate in an annual full-scale exercise that is community-based; or

(A) When a community-based exercise is not accessible, conduct an annual individual, facility-based functional exercise.

(B) If the LTC facility experiences an actual natural or man-made emergency that requires activation of the emergency plan, the LTC facility is exempt from engaging its next required a full-scale community-based or individual, facility-based functional exercise following the onset of the emergency event.

(ii) Conduct an additional annual exercise that may include, but is not limited to the following:

(A) A second full-scale exercise that is community-based or an individual, facility-based functional exercise; or
(B) A mock disaster drill; or (C) A tabletop exercise or workshop that is led by a facilitator includes a group discussion, using a narrated, clinically-relevant emergency scenario, and a set of problem statements, directed messages, or prepared questions designed to challenge an emergency plan.

(iii) Analyze the LTC facility's response to and maintain documentation of all drills, tabletop exercises, and emergency events, and revise the LTC facility's emergency plan, as needed.

40. Section 483.475 is amended by- a. Revising paragraphs (a)

introductory text, (a)(4), (b) introductory text, (c) introductory text, (d)

introductory text, and (d)(1)(ii);

- b. Adding paragraph (d)(1)(v); and

- c. Revising paragraph (d)(2).

The revisions and addition read as follows:

\section{\$483.475 Condition of participation: Emergency preparedness.}

(a) Emergency plan. The ICF/IID must develop and maintain an emergency preparedness plan that must be reviewed, and updated at least every 2 years The plan must do all of the following:

(4) Include a process for cooperation and collaboration with local, tribal, regional, State, and Federal emergency preparedness officials' efforts to maintain an integrated response during a disaster or emergency situation.

(b) Policies and procedures. The ICF/ IID must develop and implement emergency preparedness policies and procedures, based on the emergency plan set forth in paragraph (a) of this section, risk assessment at paragraph (a)(1) of this section, and the communication plan at paragraph (c) of this section. The policies and procedures must be reviewed and updated at least every 2 years. At a minimum, the policies and procedures must address the following:

(c) Communication plan. The ICF/IID must develop and maintain an emergency preparedness

communication plan that complies with Federal, State, and local laws and must be reviewed and updated at least every 2 years. The communication plan must include the following:

(d) Training and testing. The ICF/IID must develop and maintain an emergency preparedness training and testing program that is based on the emergency plan set forth in paragraph (a) of this section, risk assessment at paragraph (a)(1) of this section, policies and procedures at paragraph (b) of this section, and the communication plan at paragraph (c) of this section. The training and testing program must be reviewed and updated at least every 2 years. The ICF/IID must meet the requirements for evacuation drills and training at $\S 483.470(\mathrm{i})$.

(1) *** *

(ii) Provide emergency preparedness training at least every 2 years.

(v) If the emergency preparedness policies and procedures are significantly updated, the ICF/IID must conduct training on the updated policies and procedures.

(2) Testing. The ICF/IID must conduct exercises to test the emergency plan at least twice per year. The ICF/IID must do the following:

(i) Participate in an annual full-scale exercise that is community-based; or

(A) When a community-based exercise is not accessible, conduct an annual individual, facility-based functional exercise; or.

(B) If the ICF/IID experiences an actual natural or man-made emergency 
that requires activation of the emergency plan, the ICF/IID is exempt from engaging in its next required fullscale community-based or individual, facility-based functional exercise following the onset of the emergency event.

(ii) Conduct an additional annual exercise that may include, but is not limited to the following:

(A) A second full-scale exercise that is community-based or an individual, facility-based functional exercise; or

(B) A mock disaster drill; or

(C) A tabletop exercise or workshop that is led by a facilitator and includes a group discussion, using a narrated, clinically-relevant emergency scenario, and a set of problem statements, directed messages, or prepared questions designed to challenge an emergency plan.

(iii) Analyze the ICF/IID's response to and maintain documentation of all drills, tabletop exercises, and emergency events, and revise the ICF/IID's emergency plan, as needed

\section{PART 484-HOME HEALTH SERVICES}

41. The authority citation for part 484 continues to read as follows:

Authority: 42 U.S.C. 1302 and 1395hh unless otherwise indicated.

42. Section 484.2 is amended by adding definitions for "Pseudo-patient" and "Simulation" in alphabetical order to read as follows:

§484.2 Definitions.

Pseudo-patient means a person trained to participate in a role-play situation, or a computer-based mannequin device. A pseudo-patient must be capable of responding to and interacting with the home health aide trainee, and must demonstrate the general characteristics of the primary patient population served by the HHA in key areas such as age, frailty, functional status, and cognitive status.

Simulation means a training and assessment technique that mimics the reality of the homecare environment, including environmental distractions and constraints that evoke or replicate substantial aspects of the real world in a fully interactive fashion, in order to teach and assess proficiency in performing skills, and to promote decision making and critical thinking.

43. Section 484.50 is amended by removing and reserving paragraph (a)(3) and revising paragraph (c)(7)

introductory text.

The revision reads as follows:

$\S 484.50$ Condition of participation: Patien rights.

* * * * *

(c) **** of-

(7) Be advised, orally and in writing,

44. Section 484.80 is amended by revising paragraphs $(\mathrm{c})(1)$ and $(\mathrm{h})(3)$ to read as follows:

$\$ 484.80$ Condition of participation: Home health aide services.

(c) ***

(1) The competency evaluation must address each of the subjects listed in paragraph (b)(3) of this section. Subject areas specified under paragraphs (b)(3)(i), (iii), (ix), (x), and (xi) of this section must be evaluated by observing an aide's performance of the task with a patient or pseudo-patient. The remaining subject areas may be evaluated through written examination, oral examination, or after observation of a home health aide with a patient, or with a pseudo-patient as part of a simulation.

* * * * *

(h) ***

(3) If a deficiency in aide services is verified by the registered nurse or other appropriate skilled professional during an on-site visit, then the agency must conduct, and the home health aide must complete, retraining and a competency evaluation related to the deficient skill(s).

45. Section 484.102 is amended by- a. Revising paragraphs (a) introductory text, (a)(4), (b) introductory text, (c) introductory text, and (d) introductory text and the first paragraph (d)(1)(ii);

a b. Redesignating the second paragraph (d)(1)(ii) as paragraph (d)(1)(iv);

- c. Adding paragraph (d)(1)(v); and

- d. Revising paragraph (d)(2).

The revisions and addition read as follows:

\$484.102 Condition of participation: Emergency preparedness.

\section{* * * * * \\ (a) Emergency plan. The HHA mus} develop and maintain an emergency preparedness plan that must be reviewed, and updated at least every 2 years. The plan must do all of the following:

(4) Include a process for cooperation and collaboration with local, tribal, regional, State, and Federal emergency preparedness officials' efforts to maintain an integrated response during a disaster or emergency situation.

(b) Policies and procedures. The HHA must develop and implement emergency preparedness policies and procedures, based on the emergency plan set forth in paragraph (a) of this section, risk assessment at paragraph (a)(1) of this section, and the communication plan at paragraph (c) of this section. The policies and procedures must be reviewed and updated at least every 2 years. At a minimum, the policies and procedures must address the following:

(c) Communication plan. The HHA must develop and maintain an emergency preparedness communication plan that complies with Federal, State, and local laws and must be reviewed and updated at least every 2 years. The communication plan must include all of the following:

(d) Training and testing. The HHA must develop and maintain an emergency preparedness training and testing program that is based on the emergency plan set forth in paragraph (a) of this section, risk assessment at paragraph (a)(1) of this section, policies and procedures at paragraph (b) of this section, and the communication plan at paragraph (c) of this section. The training and testing program must be reviewed and updated at least every 2 years.

(1) * * *

(ii) Provide emergency preparedness training at least every 2 years.

(v) If the emergency preparedness policies and procedures are significantly updated, the HHA must conduct training on the updated policies and procedures.

(2) Testing. The HHA must conduct exercises to test the emergency plan at least annually. The HHA must do the following:

(i) Participate in a full-scale exercise that is community-based; or

(A) When a community-based exercise is not accessible, conduct an annual individual, facility-based functional exercise every 2 years; or.

(B) If the HHA experiences an actual natural or man-made emergency that requires activation of the emergency plan, the HHA is exempt from engaging in its next required full-scale community-based or individual, facilitybased functional exercise following the onset of the emergency event.

(ii) Conduct an additional exercise every 2 years, opposite the year the full- 
scale or functional exercise under paragraph (d)(2)(i) of this section is conducted, that may include, but is not limited to the following:

(A) A second full-scale exercise that is community-based or an individual,

facility-based functional exercise; or

(B) A mock disaster drill; or

(C) A tabletop exercise or workshop that is led by a facilitator and includes a group discussion, using a narrated, clinically-relevant emergency scenario, and a set of problem statements, directed messages, or prepared questions designed to challenge an emergency plan.

(iii) Analyze the HHA's response to and maintain documentation of all drills, tabletop exercises, and emergency events, and revise the HHA's emergency plan, as needed

\section{PART 485-CONDITIONS OF PARTICIPATION: SPECIALIZED PROVIDERS}

46. The authority citation for part 485 is revised to read as follows:

Authority: 42 U.S.C. 1302 and 1395(hh).

47. Section 485.66 is amended by revising the introductory text to read as follows:

\section{\$485.66 Condition of participation: Utilization review plan.}

The facility must have in effect a written utilization review plan that is implemented annually, to assess the necessity of services and promotes the most efficient use of services provided by the facility.

48. Section 485.68 is amended bya. Revising paragraphs (a) introductory text, (a)(4), (b) introductory text, (c) introductory text, (d) introductory text, and (d)(1)(ii);

- b. Adding paragraph (d)(1)(v); and

- c. Revising paragraph (d)(2).

The revisions and addition read as follows:

\section{\$485.68 Condition of participation: Emergency preparedness.}

(a) Emergency plan. The CORF must develop and maintain an emergency preparedness plan that must be reviewed and updated at least every 2 years. The plan must do all of the following:

(4) Include a process for cooperation and collaboration with local, tribal, regional, State, and Federal emergency preparedness officials' efforts to maintain an integrated response during a disaster or emergency situation.

(b) Policies and procedures. The CORF must develop and implement emergency preparedness policies and procedures, based on the emergency plan set forth in paragraph (a) of this section, risk assessment at paragraph (a)(1) of this section, and the communication plan at paragraph (c) of this section. The policies and procedures must be reviewed and updated at least every 2 years. At a minimum, the policies and procedures must address the following:

(c) Communication plan. The CORF must develop and maintain an emergency preparedness communication plan that complies with Federal, State, and local laws and must be reviewed and updated at least every 2 years. The communication plan must include all of the following:

(d) Training and testing. The CORF must develop and maintain an emergency preparedness training and testing program that is based on the emergency plan set forth in paragraph (a) of this section, risk assessment at paragraph (a)(1) of this section, policies and procedures at paragraph (b) of this section, and the communication plan at paragraph (c) of this section. The training and testing program must be reviewed and updated at least every 2 years.

(1) * * *

(ii) Provide emergency preparedness training at least every 2 years.

(v) If the emergency preparedness policies and procedures are significantly updated, the CORF must conduct training on the updated policies and procedures.

(2) Testing. The CORF must conduct exercises to test the emergency plan at least annually. The CORF must do the following:

(i) Participate in a full-scale exercise that is community-based every 2 years or

(A) When a community-based exercise is not accessible, conduct an individual, facility-based functional exercise every 2 years; or

(B) If the CORF experiences an actual natural or man-made emergency that requires activation of the emergency plan, the CORF is exempt from engaging in its next required community-based or individual, facility-based functional exercise following the onset of the emergency event. (ii) Conduct an additional exercise every 2 years, opposite the year the fullscale or functional exercise under paragraph (d)(2)(i) of this section is conducted, that may include, but is not limited to the following:

(A) A second full-scale exercise that is community-based or an individual, facility-based functional exercise; or

(B) A mock disaster drill; or

(C) A tabletop exercise or workshop that is led by a facilitator and includes a group discussion, using a narrated, clinically-relevant emergency scenario, and a set of problem statements, directed messages, or prepared questions designed to challenge an emergency plan.

(iii) Analyze the CORF's response to and maintain documentation of all drills, tabletop exercises, and emergency events, and revise the CORF's emergency plan, as needed.

49. Section 485.625 is amended by- a. Revising paragraphs (a) introductory text, (a)(4), (b) introductory text, (c) introductory text, (d) introductory text, and (d)(1)(ii);

- b. Adding paragraph (d)(1)(v); and

- c. Revising paragraph (d)(2).

The revisions and addition read as follows:

\section{\$485.625 Condition of participation: Emergency preparedness.}

(a) Emergency plan. The CAH must develop and maintain an emergency preparedness plan that must be reviewed and updated at least every 2 years. The plan must do all of the following:

(4) Include a process for cooperation and collaboration with local, tribal, regional, State, and Federal emergency preparedness officials' efforts to maintain an integrated response during a disaster or emergency situation.

(b) Policies and procedures. The CAH must develop and implement emergency preparedness policies and procedures, based on the emergency plan set forth in paragraph (a) of this section, risk assessment at paragraph (a)(1) of this section, and the communication plan at paragraph (c) of this section. The policies and procedures must be reviewed and updated at least every 2 years. At a minimum, the policies and procedures must address the following:

(c) Communication plan. The $\mathrm{CAH}$ must develop and maintain an emergency preparedness communication plan that complies with 
Federal, State, and local laws and must be reviewed and updated at least every 2 years. The communication plan must include all of the following:

(d) Training and testing. The CAH must develop and maintain an emergency preparedness training and testing program that is based on the emergency plan set forth in paragraph (a) of this section, risk assessment at paragraph (a)(1) of this section, policies and procedures at paragraph (b) of this section, and the communication plan at paragraph (c) of this section. The training and testing program must be reviewed and updated at least every 2 years.

(1) ***

(ii) Provide emergency preparedness training at least every 2 years.

(v) If the emergency preparedness policies and procedures are significantly updated, the CAH must conduct training on the updated policies and procedures.

(2) Testing. The CAH must conduct exercises to test the emergency plan at least twice per year. The CAH must do the following:

(i) Participate in an annual full-scale exercise that is community-based; or

(A) When a community-based exercise is not accessible, conduct an annual individual, facility-based functional exercise; or.

(B) If the CAH experiences an actual natural or man-made emergency that requires activation of the emergency plan, the CAH is exempt from engaging in its next required full-scale community-based or individual, facilitybased functional exercise following the onset of the emergency event.

(ii) Conduct an annual additional exercise, that may include, but is not limited to the following:

(A) A second full-scale exercise that is community-based or an individual, facility-based functional exercise; or

(B) A mock disaster drill; or

(C) A tabletop exercise or workshop that is led by a facilitator and includes a group discussion, using a narrated, clinically-relevant emergency scenario, and a set of problem statements, directed messages, or prepared questions designed to challenge an emergency plan.

(iii) Analyze the CAH's response to and maintain documentation of all drills, tabletop exercises, and emergency events, and revise the CAH's emergency plan, as needed.

\section{§485.627 [Amended]}

- 50. Section 485.627 is amended by removing paragraph (b)(1) and redesignating paragraphs (b)(2) and (3) as paragraphs (b)(1) and (2), respectively.

- 51. Section 485.631 is amended by adding paragraph (d) to read as follows:

\section{\$485.631 Condition of participation:} Staffing and staff responsibilities.

(d) Standard: Periodic review of clinical privileges and performance. The CAH requires that-

(1) The quality and appropriateness of the diagnosis and treatment furnished by nurse practitioners, clinical nurse specialist, and physician assistants at the CAH are evaluated by a member of the CAH staff who is a doctor of medicine or osteopathy or by another doctor of medicine or osteopathy under contract with the CAH.

(2) The quality and appropriateness of the diagnosis and treatment furnished by doctors of medicine or osteopathy at the $\mathrm{CAH}$ are evaluated by-

(i) One hospital that is a member of the network, when applicable;

(ii) One Quality Improvement Organization (QIO) or equivalent entity;

(iii) One other appropriate and qualified entity identified in the State rural health care plan;

(iv) In the case of distant-site physicians and practitioners providing telemedicine services to the CAH's patient under an agreement between the $\mathrm{CAH}$ and a distant-site hospital, the distant-site hospital; or

(v) In the case of distant-site physicians and practitioners providing telemedicine services to the CAH's patients under a written agreement between the CAH and a distant-site telemedicine entity, one of the entities listed in paragraphs (d)(2)(i) through (iii) of this section.

(3) The CAH staff consider the findings of the evaluation and make the necessary changes as specified in paragraphs (b) through (d) of this section.

- 52. Section 485.635 is amended by-

- a. Removing paragraph (a)(3)(vi);

- b. Redesignating paragraph (a)(3)(vii)

as paragraph (a)(3)(vi); and

- c. Revising newly designated

paragraph (a)(3)(vi) and paragraph (a)(4).

The revisions read as follows:

$\S 485.635$ Condition of participation:

Provision of services.

(a) ***

(3) ***

(vi) Procedures that ensure that the nutritional needs of inpatients are met in accordance with recognized dietary practices. All patient diets, including therapeutic diets, must be ordered by the practitioner responsible for the care of the patients or by a qualified dietitian or qualified nutrition professional as authorized by the medical staff in accordance with State law governing dietitians and nutrition professionals and that the requirement of $\S 483.25(i)$ of this chapter is met with respect to inpatients receiving post CAH SNF care.

(4) These policies are reviewed at least biennially by the group of professional personnel required under paragraph (a)(2) of this section and updated as necessary by the CAH.

53. Section 485.640 is added to read as follows:

\section{$\$ 485.640$ Condition of participation: Infection prevention and control and antibiotic stewardship programs.}

The CAH must have active facilitywide programs, for the surveillance, prevention, and control of HAIs and other infectious diseases and for the optimization of antibiotic use through stewardship. The programs must demonstrate adherence to nationally recognized infection prevention and control guidelines, as well as to best practices for improving antibiotic use where applicable, and for reducing the development and transmission of HAIs and antibiotic-resistant organisms. Infection prevention and control problems and antibiotic use issues identified in the programs must be addressed in coordination with the facility-wide quality assessment and performance improvement (QAPI) program.

(a) Standard: Infection prevention and control program organization and policies. The CAH must demonstrate that:

(1) An individual (or individuals), who is qualified through education, training, experience, or certification in infection prevention and control, is appointed by the governing body, or responsible individual, as the infection preventionist(s)/infection control professional(s) responsible for the infection prevention and control program and that the appointment is based on the recommendations of medical staff leadership and nursing leadership;

(2) The infection prevention and control program, as documented in its policies and procedures, employs methods for preventing and controlling the transmission of infections within the $\mathrm{CAH}$ and between the CAH and other healthcare settings;

(3) The infection prevention and control includes surveillance, 
prevention, and control of HAIs, including maintaining a clean and sanitary environment to avoid sources and transmission of infection, and that the program also addresses any infection control issues identified by public health authorities; and

(4) The infection prevention and control program reflects the scope and complexity of the CAH services provided.

(b) Standard: Antibiotic stewardship program organization and policies. The CAH must demonstrate that:

(1) An individual (or individuals), who is qualified through education, training, or experience in infectious diseases and/or antibiotic stewardship, is appointed by the governing body, or responsible individual, as the leader(s) of the antibiotic stewardship program and that the appointment is based on the recommendations of medical staff leadership and pharmacy leadership;

(2) The facility-wide antibiotic stewardship program:

(i) Demonstrates coordination among all components of the CAH responsible for antibiotic use and resistance, including, but not limited to, the infection prevention and control program, the QAPI program, the medical staff, nursing services, and pharmacy services;

(ii) Documents the evidence-based use of antibiotics in all departments and services of the $\mathrm{CAH}$; and

(iii) Documents any improvements, including sustained improvements, in proper antibiotic use;

(3) The antibiotic stewardship program adheres to nationally recognized guidelines, as well as best practices, for improving antibiotic use; and

(4) The antibiotic stewardship program reflects the scope and complexity of the CAH services provided.

(c) Standard: Leadership responsibilities. (1) The governing body, or responsible individual, must ensure all of the following:

(i) Systems are in place and operational for the tracking of all infection surveillance, prevention and control, and antibiotic use activities, in order to demonstrate the implementation, success, and sustainability of such activities.

(ii) All HAIs and other infectious diseases identified by the infection prevention and control program as well as antibiotic use issues identified by the antibiotic stewardship program are addressed in collaboration with the CAH's QAPI leadership.
(2) The infection prevention and control professional(s) is responsible for:

(i) The development and implementation of facility-wide infection surveillance, prevention, and control policies and procedures that adhere to nationally recognized guidelines.

(ii) All documentation, written or electronic, of the infection prevention and control program and its surveillance, prevention, and control activities.

(iii) Communication and collaboration with the CAH's QAPI program on infection prevention and control issues.

(iv) Competency-based training and education of CAH personnel and staff, including medical staff, and, as applicable, personnel providing contracted services in the $\mathrm{CAH}$, on the practical applications of infection prevention and control guidelines, policies and procedures.

(v) The prevention and control of HAIs, including auditing of adherence to infection prevention and control policies and procedures by CAH personnel.

(vi) Communication and collaboration with the antibiotic stewardship program.

(3) The leader(s) of the antibiotic stewardship program is responsible for:

(i) The development and implementation of a facility-wide antibiotic stewardship program, based on nationally recognized guidelines, to monitor and improve the use of antibiotics.

(ii) All documentation, written or electronic, of antibiotic stewardship program activities.

(iii) Communication and collaboration with medical staff, nursing, and pharmacy leadership, as well as the CAH's infection prevention and control and QAPI programs, on antibiotic use issues.

(iv) Competency-based training and education of CAH personnel and staff, including medical staff, and, as applicable, personnel providing contracted services in the CAHs, on the practical applications of antibiotic stewardship guidelines, policies, and procedures.

- 54. Section 485.641 is revised to read as follows:

\section{$\$ 485.641$ Condition of participation: Quality assessment and performance improvement program.}

The CAH must develop, implement, and maintain an effective, ongoing, CAH-wide, data-driven quality assessment and performance improvement (QAPI) program. The CAH must maintain and demonstrate evidence of the effectiveness of its QAPI program.

(a) Definitions. For the purposes of this section-

Adverse event means an untoward, undesirable, and usually unanticipated event that causes death or serious injury or the risk thereof.

Error means the failure of a planned action to be completed as intended or the use of a wrong plan to achieve an aim. Errors can include problems in practice, products, procedures, and systems; and

Medical error means an error that occurs in the delivery of healthcare services.

(b) Standard: QAPI Program Design and scope. The CAH's QAPI program must:

(1) Be appropriate for the complexity of the CAH's organization and services provided.

(2) Be ongoing and comprehensive.

(3) Involve all departments of the $\mathrm{CAH}$ and services (including those services furnished under contract or arrangement).

(4) Use objective measures to evaluate its organizational processes, functions and services.

(5) Address outcome indicators related to improved health outcomes and the prevention and reduction of medical errors, adverse events, $\mathrm{CAH}$ acquired conditions, and transitions of care, including readmissions.

(c) Standard: Governance and leadership. The CAH's governing body or responsible individual is ultimately responsible for the CAH's QAPI program and is responsible and accountable for ensuring that the QAPI program meets the requirements of paragraph (b) of this section.

(d) Standard: Program activities. For each of the areas listed in paragraph (b) of this section, the CAH must:

(1) Focus on measures related to improved health outcomes that are shown to be predictive of desired patient outcomes.

(2) Use the measures to analyze and track its performance.

(3) Set priorities for performance improvement, considering either highvolume, high-risk services, or problemprone areas.

(e) Standard: Program data collection and analysis. The program must incorporate quality indicator data including patient care data, and other relevant data, in order to achieve the goals of the QAPI program.

- 55. Section 485.645 is amended by-

a. Revising the introductory text;

- b. Revising paragraph (d)(1); 
c. Removing paragraph (d)(4);

- d. Redesignating paragraphs (d)(5)

through (9) as paragraphs (d)(4) through

(8), respectively; and

- e. Revising newly redesignated

paragraphs (d)(4) and (7).

The revisions read as follows:

\$485.645 Special requirements for $\mathrm{CAH}$ providers of long-term care services ("swing-beds")

A CAH must meet the following requirements in order to be granted an approval from CMS to provide post-

CAH SNF care, as specified in $\$ 409.30$ of this chapter, and to be paid for SNFlevel services, in accordance with paragraph (c) of this section.

(d) ***

(1) Resident rights (§ 483.10(b)(7), (c)(1), (c)(2)(iii), (c)(6), (d), (e)(2) and (4), (f)(4)(ii) and (iii), (g)(8) and (17), (g)(18) introductory text, and (h) of this chapter).

(4) Social services (§ $483.40(d)$ of this chapter).

* $* * * * * *{ }^{*} *$ Dental services $(\S 483.55(\mathrm{a})(2),(3)$, (4), and (5) and (b) of this chapter).

- 56. Section 485.727 is amended bya. Revising paragraphs (a) introductory text, (a)(5), (b) introductory text, (c) introductory text, (d) introductory text, and (d)(1)(ii);

- b. Adding paragraph (d)(1)(v); and

- c. Revising paragraph (d)(2).

The revisions and addition read as follows:

\section{\$485.727 Condition of participation: Emergency preparedness.}

(a) Emergency plan. The

Organizations must develop and maintain an emergency preparedness plan that must be reviewed and updated at least every 2 years. The plan must do all of the following:

(5) Include a process for cooperation and collaboration with local, tribal, regional, State, and Federal emergency preparedness officials' efforts to maintain an integrated response during a disaster or emergency situation.

(b) Policies and procedures. The Organizations must develop and implement emergency preparedness policies and procedures, based on the emergency plan set forth in paragraph (a) of this section, risk assessment at paragraph (a)(1) of this section, and the communication plan at paragraph (c) of this section. The policies and procedures must be reviewed and updated at least every 2 years. At a minimum, the policies and procedures must address the following:

(c) Communication plan. The Organizations must develop and maintain an emergency preparedness communication plan that complies with Federal, State, and local laws and must be reviewed and updated at least every 2 years. The communication plan must include all of the following:

(d) Training and testing. The Organizations must develop and maintain an emergency preparedness training and testing program that is based on the emergency plan set forth in paragraph (a) of this section, risk assessment at paragraph (a)(1) of this section, policies and procedures at paragraph (b) of this section, and the communication plan at paragraph (c) of this section. The training and testing program must be reviewed and updated at least every 2 years.

(1) * * *

(ii) Provide emergency preparedness training at least every 2 years.

(v) If the emergency preparedness policies and procedures are significantly updated, the Organizations must conduct training on the updated policies and procedures.

(2) Testing. The Organizations must conduct exercises to test the emergency plan at least annually. The Organizations must do the following:

(i) Participate in a full-scale exercise that is community-based every 2 years; or

(A) When a community-based exercise is not accessible, an individual, facility-based functional exercise every 2 years; or.

(B) If the Organizations experience an actual natural or man-made emergency that requires activation of the emergency plan, the organization is exempt from engaging in its next required full-scale community-based or individual, facility-based functional exercise following the onset of the emergency event.

(ii) Conduct an additional exercise every 2 years, opposite the year the fullscale or functional exercise under paragraph (d)(2)(i) of this section is conducted, that may include, but is not limited to the following:

(A) A second full-scale exercise that is community-based or an individual, facility-based functional exercise; or

(B) A mock disaster drill; or

(C) A tabletop exercise or workshop that is led by a facilitator and includes a group discussion, using a narrated, clinically-relevant emergency scenario, and a set of problem statements, directed messages, or prepared questions designed to challenge an emergency plan.

(iii) Analyze the Organization's response to and maintain documentation of all drills, tabletop exercises, and emergency events, and revise their emergency plan, as needed.

57. Section 485.914 is amended by revising paragraphs (d)(1) and (2) to read as follows:

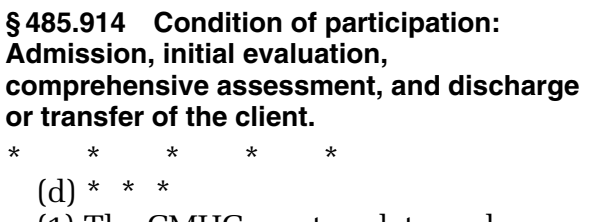

(1) The CMHC must update each client's comprehensive assessment via the CMHC interdisciplinary treatment team, in consultation with the client's primary health care provider (if any), when changes in the client's status, responses to treatment, or goal achievement have occurred and in accordance with current standards of practice.

(2) For clients that receive PHP services, the assessment must be updated no less frequently than every 30 days.

58. Section 485.920 is amended by revising paragraphs (a) introductory text, (a)(4), (b) introductory text, (c) introductory text, and (d) to read as follows:

\section{$\$ 485.920$ Condition of participation: Emergency preparedness.}

(a) Emergency plan. The CMHC must develop and maintain an emergency preparedness plan that must be reviewed, and updated at least every 2 years. The plan must do all of the following:

(4) Include a process for cooperation and collaboration with local, tribal, regional, State, and Federal emergency preparedness officials' efforts to maintain an integrated response during a disaster or emergency situation.

(b) Policies and procedures. The CMHC must develop and implement emergency preparedness policies and procedures, based on the emergency plan set forth in paragraph (a) of this section, risk assessment at paragraph (a)(1) of this section, and the communication plan at paragraph (c) of this section. The policies and 
procedures must be reviewed and updated at least every 2 years. At a minimum, the policies and procedures must address the following:

(c) Communication plan. The CMHC must develop and maintain an emergency preparedness communication plan that complies with Federal, State, and local laws and must be reviewed and updated at least every 2 years. The communication plan must include all of the following:

(d) Training and testing. The CMHC must develop and maintain an emergency preparedness training and testing program that is based on the emergency plan set forth in paragraph (a) of this section, risk assessment at paragraph (a)(1) of this section, policies and procedures at paragraph (b) of this section, and the communication plan at paragraph (c) of this section. The training and testing program must be reviewed and updated at least every 2 years. If the emergency preparedness policies and procedures are significantly updated, the CMHC must conduct training on the updated policies and procedures.

(1) Training. The CMHC must provide initial training in emergency preparedness policies and procedures to all new and existing staff, individuals providing services under arrangement, and volunteers, consistent with their expected roles, and maintain documentation of the training. The CMHC must demonstrate staff knowledge of emergency procedures. Thereafter, the CMHC must provide emergency preparedness training at least every 2 years.

(2) Testing. The CMHC must conduct exercises to test the emergency plan at least annually. The CMHC must:

(i) Participate in a full-scale exercise that is community-based every 2 years; or

(A) When a community-based exercise is not accessible, conduct an individual, facility-based every 2 years; or.

(B) If the CMHC experiences an actual natural or man-made emergency that requires activation of the emergency plan, the CMHC is exempt from engaging in its next required community-based or individual, facilitybased functional exercise following the onset of the emergency event.

(ii) Conduct an additional exercise every 2 years, opposite the year the fullscale or functional exercise under paragraph (d)(2)(i) of this section is conducted, that may include, but is not limited to following:
(A) A second full-scale exercise that is community-based or an individual, facility-based functional exercise; or

(B) A mock disaster drill; or

(C) A tabletop exercise or workshop that is led by a facilitator and includes a group discussion, using a narrated, clinically-relevant emergency scenario, and a set of problem statements, directed messages, or prepared questions designed to challenge an emergency plan.

(iii) Analyze the CMHC's response to and maintain documentation of all drills, tabletop exercises, and emergency events, and revise the CMHC's emergency plan, as needed.

\section{PART 486-CONDITIONS FOR COVERAGE OF SPECIALIZED SERVICES FURNISHED BY SUPPLIERS}

- 59. The authority citation for part 486 continues to read as follows:

Authority: 42 U.S.C. 1302, and 1395hh.

60. Section 486.104 is amended by revising paragraph (a) to read as follows:

\$486.104 Condition for coverage: Qualifications, orientation and health of technical personnel.

(a) Standard: Qualifications of technologists. All operators of the portable X-ray equipment meet the requirements of paragraph (a)(1) or (2) of this section.

(1) Successful completion of a program of formal training in X-ray technology at which the operator received appropriate training and demonstrated competence in the use of equipment and administration of portable x-ray procedures; or

(2) Successful completion of 24 full months of training and experience under the direct supervision of a physician who is certified in radiology or who possesses qualifications which are equivalent to those required for such certification. - 61. Section 486.106 is amended by revising paragraph (a)(2) to read as follows:

\$486.106 Conditions for coverage: Referral for service and preservation of records.

* * * * *

(a) ***

(2) Such physician or non-physician practitioner's order meets the requirements at $\S 410.32$ of this chapter, and includes a statement concerning the condition of the patient which indicates why portable X-ray services are necessary.

- 62. Section 486.360 is amended by-

- a. Revising paragraphs (a)

introductory text, (a)(4), (b) introductory

text, (c) introductory text, (d)

introductory text, and (d)(1)(ii);

- b. Adding paragraph (d)(1)(v); and

- c. Revising paragraph (d)(2)(i).

The revisions and addition read as follows:

\section{$\S 486.360$ Condition for Coverage: Emergency preparedness.}

(a) Emergency plan. The OPO must develop and maintain an emergency preparedness plan that must be reviewed and updated at least every 2 years. The plan must do all of the following:

(4) Include a process for cooperation and collaboration with local, tribal, regional, State, and Federal emergency preparedness officials' efforts to maintain an integrated response during a disaster or emergency situation.

(b) Policies and procedures. The OPO must develop and implement emergency preparedness policies and procedures, based on the emergency plan set forth in paragraph (a) of this section, risk assessment at paragraph (a)(1) of this section, and, the

communication plan at paragraph (c) of this section. The policies and procedures must be reviewed and updated at least every 2 years. At a minimum, the policies and procedures must address the following:

(c) Communication plan. The OPO must develop and maintain an emergency preparedness communication plan that complies with Federal, State, and local laws and must be reviewed and updated at least every 2 years. The communication plan must include all of the following:

(d) Training and testing. The OPO must develop and maintain an emergency preparedness training and testing program that is based on the emergency plan set forth in paragraph (a) of this section, risk assessment at paragraph (a)(1) of this section, policies and procedures at paragraph (b) of this section, and the communication plan at paragraph (c) of this section. The training and testing program must be reviewed and updated at least every 2 years.

$(1) * * *$ 
(ii) Provide emergency preparedness training at every 2 years.

(v) If the emergency preparedness policies and procedures are significantly updated, the OPO must conduct training on the updated policies and procedures.

(2)***

(i) Conduct a paper-based, tabletop exercise or workshop at least annually. A tabletop exercise is led by a facilitator and includes a group discussion, using a narrated, clinically-relevant emergency scenario, and a set of problem statements, directed messages, or prepared questions designed to challenge an emergency plan. If the OPO experiences an actual natural or man-made emergency that requires activation of the emergency plan, the $\mathrm{OPO}$ is exempt from engaging in its next required testing exercise following the onset of the emergency event.

\section{PART 488-SURVEY, CERTIFICATION, AND ENFORCEMENT PROCEDURES}

63. The authority citation for part 488 continues to read as follows:

Authority: 42 U.S.C. 1302 and 1395hh.

\section{\$488.30 [Amended]}

64. Section 488.30(a) is amended in the definition of "Provider of services, provider, or supplier"' by removing the phrase "transplant centers" and adding in its place the phrase "transplant programs".

65. Section 488.61 is amended-

- a. By revising the section heading;

- b. In the introductory text by removing the phrase "transplant centers" and adding in its place the phrase "transplant programs";

- c. In paragraph (a) introductory text by removing the words "centers" and "center" each time they appear and adding in their place the words "programs", and "program," respectively;

a d. In paragraph (a)(2) by removing the phrase "Scientific Registry of Transplant Beneficiary (SRTR) centerspecific" and adding in its place the phrase "Scientific Registry of Transplant Recipient (SRTR) programspecific";

- e. By revising paragraph (a)(5);

- f. By removing paragraph (c);

- g. By redesignating paragraphs (d) through (h) as paragraphs (c) through (g), respectively;

- h. By revising newly redesignated paragraphs (c) and (d), the newly redesignated paragraph (e) subject heading, and newly redesignated paragraphs (e)(1) introductory text, (e)(1)(iv), (e)(3), and (f)(1)(i) through

(iii); and

- i. In newly redesignated paragraph

$(g)(1)(x)$ by removing the reference "paragraphs (h)(1)(v), (h)(1)(vi), (h)(1)(vii) or (h)(1)(viii)" and adding in its place the reference "paragraph

(g)(1)(v), (vi), (vii) or (viii)"

The revisions read as follows:

§488.61 Special procedures for approval and re-approval of organ transplant programs.

* * * * *

(a) ***

(5) If CMS determines that a transplant program has met the data submission, clinical experience, and outcome requirements, CMS will review the program's compliance with the conditions of participation contained at $\S \S 482.72$ through 482.76 and $\S \S 482.90$ through 482.104 of this chapter using the procedures described in subpart A of this part. If the transplant program is found to be in compliance with all the conditions of participation at $\S \S 482.72$ through 482.104 of this chapter, CMS will notify the transplant program in writing of the effective date of its Medicare-approval. CMS will notify the transplant program in writing if it is not Medicare-approved.

(c) Loss of Medicare approval. Programs that have lost their Medicare approval may seek re-entry into the Medicare program at any time. A program that has lost its Medicare approval must:

(1) Request initial approval using the procedures described in paragraph (a) of this section;

(2) Be in compliance with $\S \S 482.72$ through 482.104 of this chapter at the time of the request for Medicare approval; and

(3) Submit a report to CMS

documenting any changes or corrective actions taken by the program as a result of the loss of its Medicare approval status.

(d) Transplant program inactivity. A transplant program may remain inactive and retain its Medicare approval for a period not to exceed 12 months. A transplant program must notify CMS upon its voluntary inactivation as required by $\S 482.74(\mathrm{a})(3)$ of this chapter.

(e) Consideration of mitigating factors in initial approval survey, certification, and enforcement actions for transplant programs-(1) Factors. Except for situations of immediate jeopardy or deficiencies other than failure to meet requirements at $\S 482.80$ of this chapter, CMS will consider such mitigating factors as may be appropriate in light of the nature of the deficiency and circumstances, including (but not limited to) the following, in making a decision of initial approval of a transplant program that does not meet the data submission, clinical experience, or outcome requirements:

\section{(iv) Program improvements that} substantially address root causes of graft failures or patient deaths, that have been implemented and institutionalized on a sustainable basis, and that are supported by outcomes more recent than the latest available SRTR report, for which there is a sufficient post-transplant patient and graft survival period and a sufficient number of transplants such that CMS finds that the program demonstrates present-day compliance with the requirements at $\S 482.80(\mathrm{c})(2)(\mathrm{ii})(\mathrm{C})$ of this chapter;

(3) Timing. Within 14 calendar days after CMS has issued formal written notice of a condition-level deficiency to the program, CMS must receive notification of the program's intent to seek mitigating factors approval, and receive all information for consideration of mitigating factors within 120 calendar days of the CMS written notification for a deficiency due to data submission, clinical experience or outcomes at $\S 482.80$ of this chapter. Failure to meet these timeframes may be the basis for denial of mitigating factors. CMS may permit an extension of the timeline for good cause, such as a declared public health emergency.

(f) ***

(1) ***

(i) Approve initial approval of a program's Medicare participation based upon approval of mitigating factors.

(ii) Deny the program's request for Medicare approval based on mitigating factors.

(iii) Offer a time-limited Systems Improvement Agreement, in accordance with paragraph (g) of this section, when a transplant program has waived its appeal rights, has implemented substantial program improvements that address root causes and are institutionally supported by the hospital's governing body on a sustainable basis, and has requested more time to design or implement additional improvements or demonstrate compliance with CMS outcome requirements. Upon completion of the Systems Improvement Agreement or a CMS finding that the hospital has failed to meet the terms of the Agreement, CMS makes a final determination of whether to approve or deny a program's request for Medicare 
approval based on mitigating factors. A Systems Improvement Agreement follows the process specified in paragraph (g) of this section.

\section{PART 491-CERTIFICATION OF CERTAIN HEALTH FACILITIES}

66. The authority citation for part 491 is revised to read as follows:

Authority: 42 U.S.C. 263a and 1302.

67. Section 491.9 is amended by revising paragraph (b)(4) to read as follows:

\section{\$491.9 Provision of services.}

(b) ***

(4) These policies are reviewed at least biennially by the group of professional personnel required under paragraph (b)(2) of this section and reviewed as necessary by the RHC or FQHC.

68. Section 491.11 is amended by revising paragraph (a) to read as follows:

\section{\$491.11 Program evaluation.}

(a) The clinic or center carries out, or arranges for, a biennial evaluation of its total program.

69. Section 491.12 is amended by- a. Revising paragraphs (a)

introductory text, (a)(4), (b) introductory text, (c) introductory text, (d)

introductory text, and (d)(1)(ii);

- b. Adding paragraph (d)(1)(v); and

- c. Revising paragraph (d)(2).

The revisions and addition read as follows:

\section{$\S 491.12$ Emergency preparedness.}

(a) Emergency plan. The RHC or FQHC must develop and maintain an emergency preparedness plan that must be reviewed and updated at least every 2 years. The plan must do all of the following:

(4) Include a process for cooperation and collaboration with local, tribal, regional, State, and Federal emergency preparedness officials' efforts to maintain an integrated response during a disaster or emergency situation.

(b) Policies and procedures. The RHC or FQHC must develop and implement emergency preparedness policies and procedures, based on the emergency plan set forth in paragraph (a) of this section, risk assessment at paragraph (a)(1) of this section, and the communication plan at paragraph (c) of this section. The policies and procedures must be reviewed and updated at least every 2 years. At a minimum, the policies and procedures must address the following:

(c) Communication plan. The RHC or FQHC must develop and maintain an emergency preparedness communication plan that complies with Federal, State, and local laws and must be reviewed and updated at least every 2 years. The communication plan must include all of the following:

(d) Training and testing. The RHC or FQHC must develop and maintain an emergency preparedness training and testing program that is based on the emergency plan set forth in paragraph (a) of this section, risk assessment at paragraph (a)(1) of this section, policies and procedures at paragraph (b) of this section, and the communication plan at paragraph (c) of this section. The training and testing program must be reviewed and updated at least every 2 years.

(1) * * *

(ii) Provide emergency preparedness training at least every 2 years.

(v) If the emergency preparedness policies and procedures are significantly updated, the RHC/FQHC must conduct training on the updated policies and procedures.

(2) Testing. The RHC or FQHC must conduct exercises to test the emergency plan at least annually. The RHC or FQHC must do the following:

(i) Participate in a full-scale exercise that is community-based every 2 years; or

(A) When a community-based exercise is not accessible, an individual, facility-based functional exercise every 2 years; or.

(B) If the RHC or FQHC experiences an actual natural or man-made emergency that requires activation of the emergency plan, the RHC or FQHC is exempt from engaging in its next required full-scale community-based or individual, facility-based functional exercise following the onset of the emergency event.

(ii) Conduct an additional exercise every 2 years, opposite the year the fullscale or functional exercise under paragraph (d)(2)(i) of this section is conducted, that may include, but is not limited to following:

(A) A second full-scale exercise that is community-based or an individual, facility-based functional exercise; or

(B) A mock disaster drill; or

(C) A tabletop exercise or workshop that is led by a facilitator and includes a group discussion, using a narrated, clinically-relevant emergency scenario, and a set of problem statements, directed messages, or prepared questions designed to challenge an emergency plan.

(iii) Analyze the RHC or FQHC's response to and maintain documentation of all drills, tabletop exercises, and emergency events, and revise the RHC or FQHC's emergency plan, as needed.

\section{PART 494-CONDITIONS FOR COVERAGE FOR END-STAGE RENAL DISEASE FACILITIES}

70. The authority citation for part 494 is revised to read as follows:

Authority: 42 U.S.C. 1302 and 1395hh.

71. Section 494.60 is amended by revising paragraphs (d)(1), (2), and (4) and adding paragraphs (d)(5), (e), and (f) to read as follows:

\section{$\$ 494.60$ Condition: Physical environment.}

(d) ***

(1) Except as provided in paragraph (d)(2) of this section, dialysis facilities that do not provide one or more exits to the outside at grade level from the patient treatment area level must comply with provisions of the Life Safety Code (NFPA 101 and its Tentative Interim Amendments TIA 121, TIA 12-2, TIA 12-3, and TIA 12-4) applicable to Ambulatory Health Care Occupancies, regardless of the number of patients served.

(2) Notwithstanding paragraph (d)(1) of this section, dialysis facilities participating in Medicare as of October 14, 2008 that require sprinkler systems are those housed in multi-story buildings construction Types II(000), III(200), or V(000), as defined in the Life Safety Code, section 21.1.6.1, which were constructed after January 1, 2008, and those housed in high rise buildings over 75 feet in height, which were constructed after January 1, 2008.

(4) In consideration of a recommendation by the State survey agency or at the discretion of the Secretary, the Secretary may waive, for periods deemed appropriate, specific provisions of the Life Safety Code, which would result in unreasonable hardship upon an ESRD facility, but only if the waiver will not adversely affect the health and safety of the patients.

(5) No dialysis facility may operate in a building that is adjacent to an industrial high hazard area, as described 
in sections 20.1.3.7 and 21.1.3.7 of the Health Care Facilities Code (NFPA 99 and its Tentative Interim Amendments TIA $12-2$, TIA $12-3$, TIA $12-4$, TIA $12-$ 5 , and TIA 12-6).

(e) Standard: Building safety. (1) Dialysis facilities that do not provide one or more exits to the outside at grade level from the patient treatment area level must meet the applicable provisions of the Health Care Facilities Code, regardless of the number of patients served.

(2) Chapters 7, 8, 12, and 13 of the Health Care Facilities Code do not apply to a dialysis facility.

(3) If application of the Health Care Facilities Code would result in unreasonable hardship for the dialysis facility, CMS may waive specific provisions of the Health Care Facilities Code for such facility, but only if the waiver does not adversely affect the health and safety of patients.

(f) Incorporation by reference. The standards incorporated by reference in this section are approved for incorporation by reference by the Director of the Office of the Federal Register in accordance with 5 U.S.C. 552(a) and 1 CFR part 51. You may obtain the material from the sources listed below. You may inspect a copy at the CMS Information Resource Center, 7500 Security Boulevard, Baltimore, MD or at the National Archives and Records Administration (NARA). For information on the availability of this material at NARA, email fedreg.legal@ nara.gov, or go to: www.archives.gov/ federal_register/cfr/ibr-locations.html. If any changes in the editions of the Codes are incorporated by reference, CMS will publish a document in the Federal Register to announce the changes.

(1) National Fire Protection

Association, 1 Batterymarch Park, Quincy, MA 02169, www.nfpa.org, 1617-770-3000.

(i) NFPA 99, Health Care Facilities Code, 2012 edition, issued August 11 2011.

(ii) TIA 12-2 to NFPA 99, issued August 11, 2011.

(iii) TIA $12-3$ to NFPA 99, issued August 9, 2012.

(iv) TIA $12-4$ to NFPA 99, issued March 7, 2013.

(v) TIA 12-5 to NFPA 99, issued August 1, 2013.

(vi) TIA $12-6$ to NFPA 99, issued March 3, 2014.

(vii) NFPA 101, Life Safety Code, 2012 edition, issued August 11, 2011.

(viii) TIA $12-1$ to NFPA 101, issued August 11, 2011.

(ix) TIA $12-2$ to NFPA 101, issued October 30, 2012. (x) TIA 12-3 to NFPA 101, issued

October 22, 2013.

(xi) TIA 12-4 to NFPA 101, issued

October 22, 2013.

(2) [Reserved]

72. Section 494.62 is amended by- a. Revising paragraphs (a)

introductory text, (a)(4), (b) introductory text, (c) introductory text, (d)

introductory text, and (d)(1)(ii);

- b. Adding paragraph (d)(1)(vii); and

- c. Revising paragraph (d)(2).

The revisions and addition read as follows:

\section{\$494.62 Condition of participation: Emergency preparedness.}

(a) Emergency plan. The dialysis facility must develop and maintain an emergency preparedness plan that must be evaluated and updated at least every 2 years. The plan must do all of the following:

(4) Include a process for cooperation and collaboration with local, tribal, regional, State, and Federal emergency preparedness officials' efforts to maintain an integrated response during a disaster or emergency situation. The dialysis facility must contact the local emergency preparedness agency at least annually to confirm that the agency is aware of the dialysis facility's needs in the event of an emergency.

(b) Policies and procedures. The dialysis facility must develop and implement emergency preparedness policies and procedures, based on the emergency plan set forth in paragraph (a) of this section, risk assessment at paragraph (a)(1) of this section, and the communication plan at paragraph (c) of this section. The policies and procedures must be reviewed and updated at least every 2 years. These emergencies include, but are not limited to, fire, equipment or power failures, care-related emergencies, water supply interruption, and natural disasters likely to occur in the facility's geographic area. At a minimum, the policies and procedures must address the following:

(c) Communication plan. The dialysis facility must develop and maintain an emergency preparedness

communication plan that complies with Federal, State, and local laws and must be reviewed and updated at least every 2 years. The communication plan must include all of the following:

(d) Training, testing, and orientation. The dialysis facility must develop and maintain an emergency preparedness training, testing and patient orientation program that is based on the emergency plan set forth in paragraph (a) of this section, risk assessment at paragraph (a)(1) of this section, policies and procedures at paragraph (b) of this section, and the communication plan at paragraph (c) of this section. The training, testing, and patient orientation program must be evaluated and updated at least every 2 years.

(1) * * *

(ii) Provide emergency preparedness training at least every 2 years.

(vii) If the emergency preparedness policies and procedures are significantly updated, the dialysis facility must conduct training on the updated policies and procedures.

(2) Testing. The dialysis facility must conduct exercises to test the emergency plan at least annually. The dialysis facility must do all of the following:

(i) Participate in a full-scale exercise that is community-based every 2 years; or

(A) When a community-based exercise is not accessible, an individual, and a facility-based functional exercise every 2 years; or

(B) If the dialysis facility experiences an actual natural or man-made emergency that requires activation of the emergency plan, the dialysis facility is exempt from engaging in its next required full-scale community-based or individual, facility-based functional exercise following the onset of the emergency event.

(ii) Conduct an additional exercise every 2 years, opposite the year the fullscale or functional exercise under paragraph (d)(2)(i) of this section is conducted, that may include, but is not limited to the following:

(A) A second full-scale exercise that is community-based or an individual, facility-based functional exercise; or

(B) A mock disaster drill; or

(C) A tabletop exercise or workshop that is led by a facilitator and includes a group discussion, using a narrated, clinically-relevant emergency scenario, and a set of problem statements, directed messages, or prepared questions designed to challenge an emergency plan.

(iii) Analyze the dialysis facility's response to and maintain documentation of all drills, tabletop exercises, and emergency events, and revise the dialysis facility's emergency plan, as needed. 
Dated: September 6, 2019.

Seema Verma,

Administrator, Centers for Medicare \&

Medicaid Services.

Dated: September 17, 2019.

Alex M. Azar II,

Secretary, Department of Health and Human

Services.

[FR Doc. 2019-20736 Filed 9-25-19; 11:15 am]

BILLING CODE 4120-01-P 